\title{
THE STRUCTURE AND DYNAMICS OF HOT JUPITER UPPER
} ATMOSPHERES

\author{
George Bert Trammell IV \\ West Chester, Pennsylvania
}

B.S., Astronomy \& Astrophysics, Pennsylvania State University, 2005

B.S., Physics, Pennsylvania State University, 2005

M.S., Astronomy, University of Virginia, 2008
A Dissertation Presented to the Graduate
Faculty of the University of Virginia in Candidacy for the Degree of
Doctor of Philosophy

\author{
Department of Astronomy \\ University of Virginia \\ August, 2013
}

Philip L. Arras

Zhi-Yun Li

D. Mark Whittle

Roger A. Chevalier

Andrew S. Grimshaw 


\begin{abstract}
Gas giant exoplanets orbiting close to their parent stars ("hot Jupiters") experience radiation and stellar wind flux $\sim 10^{4}$ times higher than solar system giants. Energy deposited at high altitude heats and ionizes their upper atmospheres, where densities are sufficiently low that magnetic forces can dominate the dynamics of the gas physics that previous studies of their upper atmospheres have largely ignored. High levels of extreme-ultraviolet radiation deposited into the upper atmosphere inflates the scale height, making the upper atmosphere of hot Jupiters that transit the disk of their host stars potentially observable through transmission spectroscopy of atomic resonance lines.
\end{abstract}

Motivated by the $\simeq 10 \%$ decrease in hydrogen $(\mathrm{H})$ Lyman $\alpha$ flux observed for the hot Jupiter HD 209458b, and the interpretations in the literature that the absorbing neutral H gas lies outside the planet's Roche Lobe and may be escaping, I perform semi-analytic calculations and 2D magnetohydrodynamical (MHD) simulations of photoionization-driven escape of gas from the planet. The high ionization levels expected in the upper atmosphere imply that any outflow would be well-coupled to the planetary magnetic field. I have constructed the first models of the upper atmosphere that include the effects of the intrinsic planetary magnetic field and the stellar tide. The solutions exhibit the following three features: (1) a region near the equator of static, magnetically-confined gas, (2) a transonic outflow at mid-latitudes in a magnetically-channeled wind zone, and (3) a region near the poles where outflow can be quenched by a sufficiently strong stellar tide.

Using the magnetized wind model, I compute Lyman $\alpha$ transit profiles using several different simulation parameters, to compare with available observational data for the hot Jupiters HD 209458b and HD 189733b. I also use the consistency with obser- 
vations to offer an alternative to the simpler, hydrodynamic escape interpretation for extended $\mathrm{H}$ absorption seen in the transmission spectra of highly irradiated gas giants such as HD 209458b. The results demonstrate (1) the importance of magnetic forces and stellar tidal forces for an accurate determination of mass and angular momentum loss rates, and (2) absorption in the Lyman $\alpha$ line at $\pm 100 \mathrm{~km} \mathrm{~s}^{-1}$ from line center can occur from regions outside the planet's Roche Lobe without requiring mass loss to occur at all latitudes. Mass and angular momentum loss rates, which are not directly accessible through observations, determine if significant atmospheric "evaporation" and/or deviation from tidal synchronization occurs on Gyr timescales.

The utility of a model for magnetized upper atmospheres of hot Jupiters can be extended to additional classes of exoplanets, such as hot Neptunes, which are Neptune-sized planets with tight orbits around their parent stars, and perhaps even super Earths $\left(M_{\oplus} \lesssim M \lesssim 10 M_{\oplus}\right)$. A future refinement of the model is to include the stellar wind contribution, which sets the outer boundary for planetary atmospheres. 


\section{Acknowledgements}

I wish to sincerely thank my research advisors, Phil Arras and Zhi-Yun Li, for their extraordinary commitment to me, and for playing such an inspirational role in navigating a diverse, challenging, and simultaneously exciting topic that lies at the frontier of astronomical research. I will always be very grateful for their patience, continued support and dedication throughout my time at the University of Virginia and beyond, without which this project would have never been possible. Their consistent encouragement for me to better understand the problem significantly increased the scientific merit of my project, while also inspiring me to never stop asking questions. I also thank them for their kindness, their willingness to offer advice, and their unending desire to see me succeed.

I also thank my family, not only for their continued support throughout the most challenging period of my life, but also for their love and their embrace of my career. Their excitement and their enthusiasm has been invaluable to me. I am also grateful to my beautiful wife Janet for her unending support and love through all of the challenges and all of the hard work, without whom I would not be where I am today. And finally, I also wish to thank others who provided valuable support and advice, including Mark Whittle, Aaron Evans, Edward Murphy, and John Hawley. 
For Ross 


\section{Table of contents}

Abstract

Acknowledgements

1 General Introduction 1

1.1 Overview and Context . . . . . . . . . . . . . . . . 1

1.2 Upper Atmosphere Observations . . . . . . . . . . . . . . . 5

1.2 .1 HD 209458b . . . . . . . . . . . . . . . 7

1.2 .2 HD $189733 \mathrm{~b} \ldots \ldots \ldots \ldots$

1.2.3 Towards a Model for the Upper Atmosphere . . . . . . . . . . 10

1.2.4 Expected Magnetic Field Strengths . . . . . . . . . . . . . . 13

1.2.5 Connection to Roche Lobe Overflow Model . . . . . . . . . . . 14

1.3 Approach \& Methodology . . . . . . . . . . . . . . . . . . 15

2 An MHD Model of the Upper Atmosphere 17

2.1 Overview . . . . . . . . . . . . . . . . . . . . 17

2.2 Problem Setup \& Approximations . . . . . . . . . . . . . . . . . 18

2.3 Magnetic Field Strengths . . . . . . . . . . . . . . . . . . . . . 21

2.4 Dead-Zone/Wind-Zone Structure of the Upper Atmosphere . . . . . 23

2.5 Tidal Forces \& Magnetic Geometry . . . . . . . . . . . . . . . . . . 26

2.6 The Dead Zone . . . . . . . . . . . . . . . . . . . . . . . . 29

2.7 The Wind Zone . . . . . . . . . . . . . . . . . . . . . . . . . . 37

$2.8 \mathrm{H}$ and $\mathrm{H}^{+}$Layers: A Simplified 1D Thermal Model . . . . . . . . . . 46

2.9 Global Models . . . . . . . . . . . . . . . . . . . 53

2.10 Mass Loss Rates \& Spin-Down Torque . . . . . . . . . . . . . . . . . 59

2.11 Neutral H Column Densities . . . . . . . . . . . . . . . . . . 62

2.12 Lyman $\alpha$ Transmission Spectra . . . . . . . . . . . . . . . . . . . . . . 65

2.13 Comparison to Roche-Lobe Overflow . . . . . . . . . . . . . . . . . 74

2.14 Summary . . . . . . . . . . . . . . . . . . . 75

2.15 Appendix: MHD Wind Equations . . . . . . . . . . . . . . 79

2.16 Appendix: Mean Free Paths, Ion-Neutral Drift \& Ohm's law . . . . . 84 
3 Numerical Simulations with ZEUS-MP

3.1 Overview . . . . . . . . . . . . . . . . . . . . . 92

3.2 Relevant Equations . . . . . . . . . . . . . . . . . . . . 94

3.3 Numerical Methods . . . . . . . . . . . . . . . . . . . . 95

3.4 Time Step Control . . . . . . . . . . . . . . . . . . . . . . . . 98

3.5 Staggered Grid Topology \& Workflow . . . . . . . . . . . . . . . . . . 100

3.6 Example Numerical Tests . . . . . . . . . . . . . . . . . . . . . . 103

3.6.1 Sedov-Taylor Blast Wave . . . . . . . . . . . . . . . . . . . . . 104

3.6.2 Magnetic Braking of an Aligned Rotor . . . . . . . . . . . . . 106

3.6.3 Spherical Hydrodynamic Wind . . . . . . . . . . . . 107

4 MHD Simulations of Hot Jupiter Upper Atmospheres 112

4.1 Description of the Simulations . . . . . . . . . . . . . . . . . . 112

4.2 Simulation Results . . . . . . . . . . . . . . . . . . . . . 119

4.2.1 Qualitative Results: Magnetic Field and Tidal Strength . . . . 119

4.2.2 Quantitative Analysis: Density and Velocity Profiles . . . . . 126

4.2.3 Mass and Angular Momentum Loss Rates . . . . . . . . . . . . 127

4.3 Transit Depths in Ly $\alpha \ldots \ldots$. . . . . . . . . . . . . . . . . 131

4.3.1 Computing the Transit Profiles . . . . . . . . . . . . . 132

4.3.2 Results for HD 209458b . . . . . . . . . . . . . . . 134

4.3.3 Results for HD 189733b . . . . . . . . . . . . . 139

4.4 Summary . . . . . . . . . . . . . . . . . . . . . . . . 139

4.5 Appendix: Shear Layer and Current Sheet Near the Equatorial DeadZone/Wind-Zone Boundary . . . . . . . . . . . . . . . 141

5 Summary \& Future Work $\quad 153$

5.1 Analytic MHD Models . . . . . . . . . . . . . . . . . . . 154

5.2 MHD Simulation Results . . . . . . . . . . . . . . . . . 155

5.3 Extension to 3D-MHD Simulations . . . . . . . . . . . . . 156

5.4 Additional 3D-Model Applications . . . . . . . . . . . . . . . . . . 157

5.4.1 The Stellar Wind Contribution _. . . . . . . . . . . . . . 157

5.4 .2 Perceived Impact . . . . . . . . . . . . . . . . . . . . 158 


\section{Chapter 1}

\section{General Introduction}

\subsection{Overview and Context}

To date, there are more than 700 confirmed detections of planets orbiting other stars. With more than 3150 additional unconfirmed planet candidates from NASA's Kepler Mission $^{1}$, whose primary mission has just now drawn to a close, the dataset of exoplanets is growing as more and more planet candidates are confirmed. Figure 1.1 shows the minimum planet mass $M \sin i$ vs. orbital distance distribution of all confirmed planets as of 20 July 2013 from the Exoplanet Data Explorer portal ${ }^{2}$. The range of several decades in minimum mass and orbital distances illustrates the diversity of exoplanetary systems. The color scale represents the heliocentric distance to the host star, where it is clear that nearly all of the confirmed exoplanets are within $\lesssim 100$ pc of the Sun. A particularly interesting subset of these confirmed exoplanets is Jupiter-class giants (EGPs), which dominates the population and occupies a region of the plot with $M \sin i \gtrsim 0.5 M_{\mathrm{Jup}}$. The blue histogram in Figure 1.2 shows the ob-

\footnotetext{
${ }^{1}$ http://kepler.nasa.gov

${ }^{2}$ http://exoplanets.org
} 


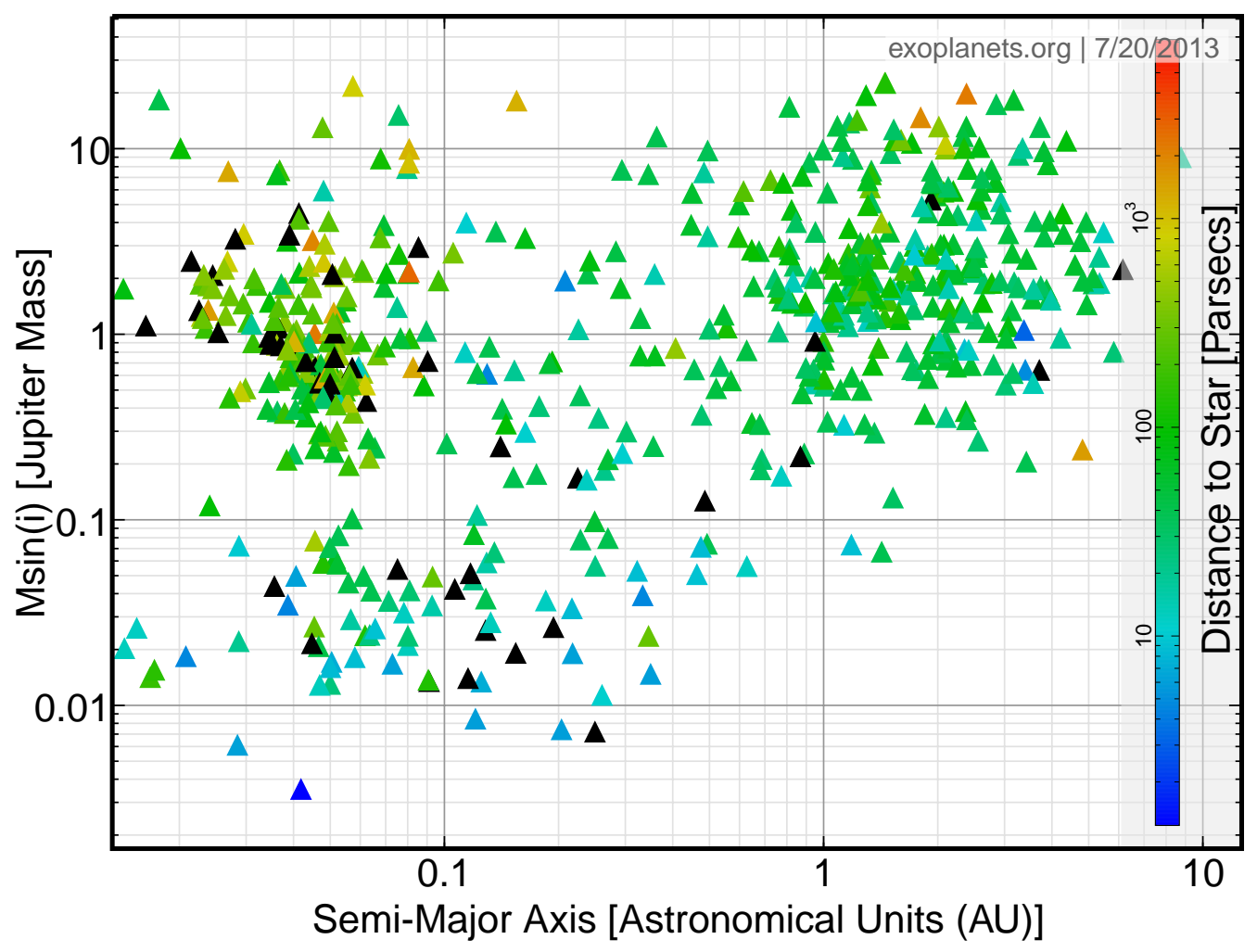

Fig. 1.1. - Mass vs. orbit size for all 723 confirmed exoplanet detections as of 20 July 2013 orbiting normal stars from the Exoplanet Orbit Database.

served orbital period distribution for the planets shown in Figure $1.1(N=711)$. The small peak in green at periods $P \sim 10^{3}-10^{4} \mathrm{~d}$ highlights the Jupiter analogs (i.e., $M \sin i>0.5 M_{\mathrm{Jup}}$ and $P>8 \mathrm{yr}$ ), while the larger subset in red represents the "hot Jupiters" (i.e., $M \sin i>0.5 M_{\text {Jup }}$ and $P<10$ d; hereafter "HJs"). The discovery of $>152$ HJs to date illustrate one fundamental difference in the architecture of extrasolar planets, in that no such analog exists within our own solar system. The planets that are orbiting their host stars with $P \sim$ days are much closer to their host stars. For example, for a HJ with orbital distance $a \sim 0.05 \mathrm{AU}$ (astronomical units), the radiation and stellar wind flux $F$ are a factor $\sim\left(a / a_{\text {Jup }}\right)^{2} \simeq(0.05 / 5.2)^{2} \sim 10^{4}$ times higher compared to that experienced by Jupiter. Therefore, one might expect that the structure of their atmospheres as well as their dynamical history and evolution 


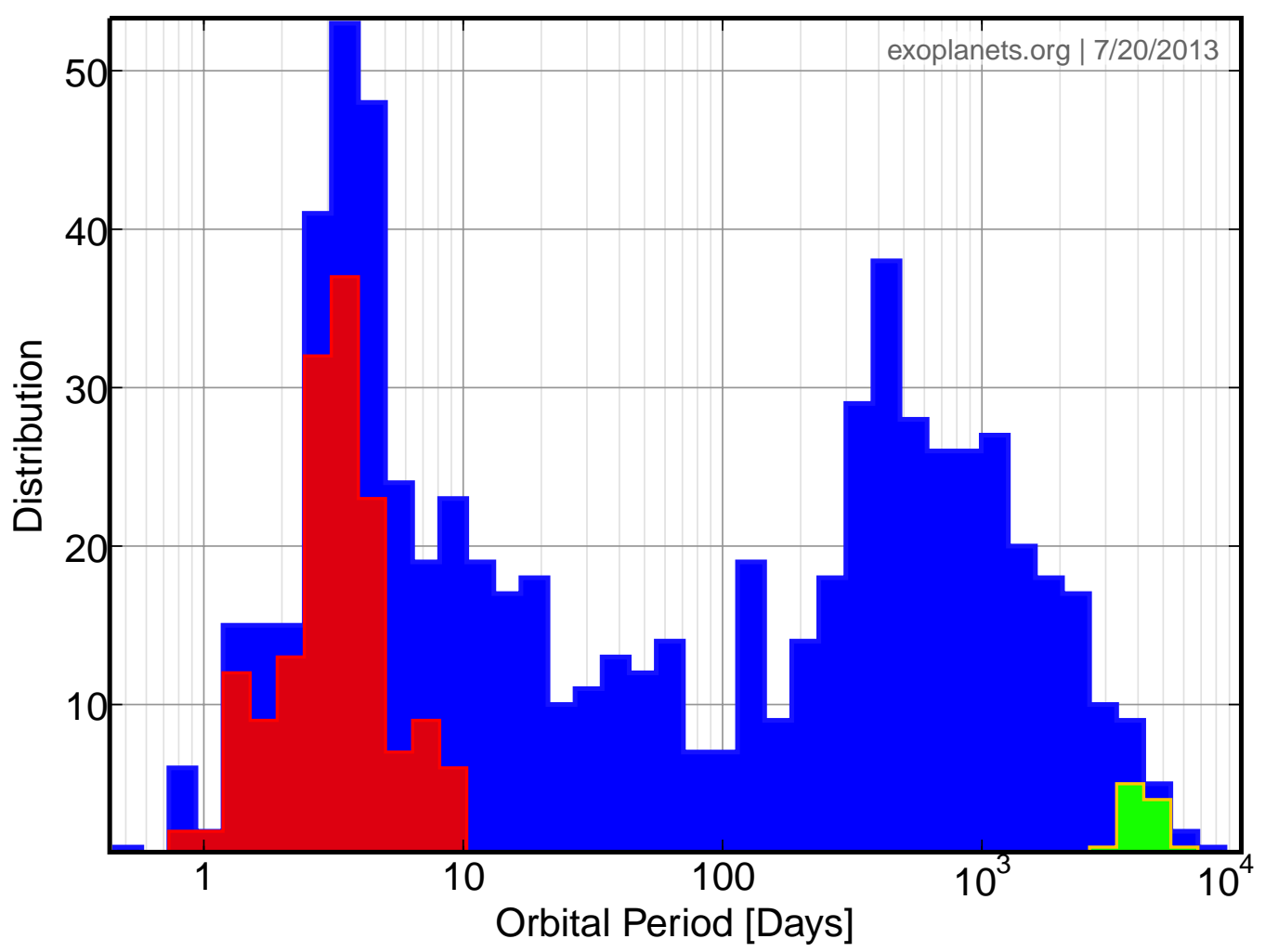

Fig. 1.2.- Orbital period $P$ distribution for 711 confirmed detections of planets in Figure 1.1 (blue) in comparison with 11 Jupiter-like orbits and masses (green) and 152 hot Jupiters (red).

might be fundamentally different from that of Jupiter and Saturn due to increased heating.

Figure 1.3 shows the distribution of orbital distances for only the hot Jupiter subclass of exoplanets (red histogram in Figure 1.2). For a Sun-like star with radius $R_{\mathrm{s}}=7 \times 10^{10} \mathrm{~cm}$, a value of $a \simeq 0.05$ AU corresponds to a mere $10 R_{\mathrm{s}}$. Therefore, we expect that any study of HJs must necessarily consider the higher intensity stellar wind, and higher extreme-ultraviolet (EUV) flux, and even host stellar tidal acceleration. Given the significant representation of HJs within the overall observed exoplanet population, a more detailed understanding is of high scientific value. Furthermore, the HJs are a class of exoplanets that have no analog in our own solar system, making 
their study an entirely new field of exoplanet research.

For those planets which cross the disk of their host stars as seen from Earth, transmission spectroscopy can reveal the fingerprint of their atmospheres on the spectrum of the host star. Gas giants with tight orbits posses two distinct advantages that facilitate their detection - (1) their higher orbital frequency $\Omega=\left(G M_{\text {star }} / a^{3}\right)^{1 / 2}$ is a strong function of $a$, and so the transit of a planet with a tighter orbit (i.e., smaller $a$ ) has a higher probability to be observed by chance, and (2) their larger size increases the area relative to the stellar disk. Therefore, it is no surprise that one particular area of rapid progress has been the studies of HJ atmospheres.

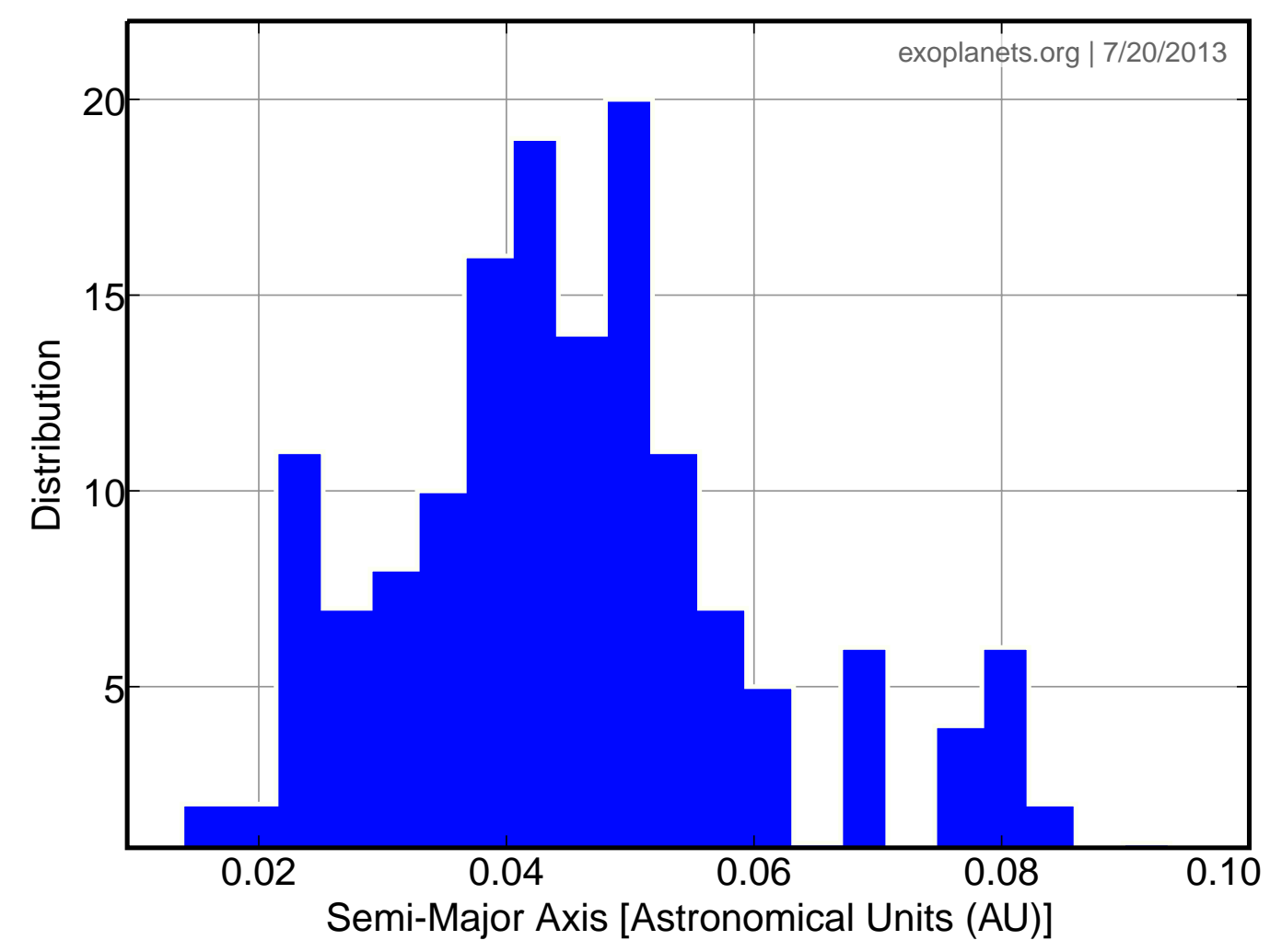

Fig. 1.3.- Orbital distance distribution for 152 HJs highlighted as the red histogram in Figure 1.2 above. 


\subsection{Upper Atmosphere Observations}

Because of a lack of a similar analog in our own solar system, HJs represent an opportunity to test theoretical predictions of the structure and the dynamics of planetary atmospheres in the extreme environment close to a parent star. The tenuous upper atmosphere of these planets is most directly exposed to this environment, at an altitude that is far above layers probed by optical continuum observations that are the primary signal detection method via transits. In contrast are our solar system gas giants, which orbit at relatively large distances and are thus relatively cold, having an atmospheric scale height that is geometrically thin compared to a planetary radius. For example, for a pure $\mathrm{H}$ atmosphere with mean molecular weight $\mu=m_{\mathrm{H}}$ at Jupiter's temperature $T \sim 100 \mathrm{~K}$ and specific gravity $g_{\text {Jup }}$, the atmospheric scale height $H$ is given by

$$
\begin{aligned}
H & =\frac{k_{B} T}{\mu g_{\mathrm{Jup}}} \\
& =\frac{k_{B} T R_{\mathrm{Jup}}^{2}}{\mu G M_{\mathrm{Jup}}} \\
\therefore H & \simeq 5 \times 10^{-4} R_{\mathrm{Jup}} .
\end{aligned}
$$

A key difference for HJs is that the absorption of higher ionizing EUV and Xray flux from the host star can heat their upper atmospheres to higher temperatures $\left(T \gtrsim 10^{4} \mathrm{~K}\right)$, which dramatically inflates those layers to scale heights that become a significant fraction $\sim 10^{-2}-10^{-1}$ of a planetary radius. This makes the atmosphere easier to detect during transit, since the increased area obscures a higher fraction of the star's disk. This sub-class of transiting HJs thus provides most of the information about their atmospheres, because the composition and structure of the planetary atmosphere are imprinted on the stellar spectrum via resonance absorption lines. An 
example theoretical optical light curve for HD 209458b is shown in Figure 1.4, where the star and planet are shown to scale and the planet trajectory through transit is shown by a dotted line that represents the measured impact parameter.

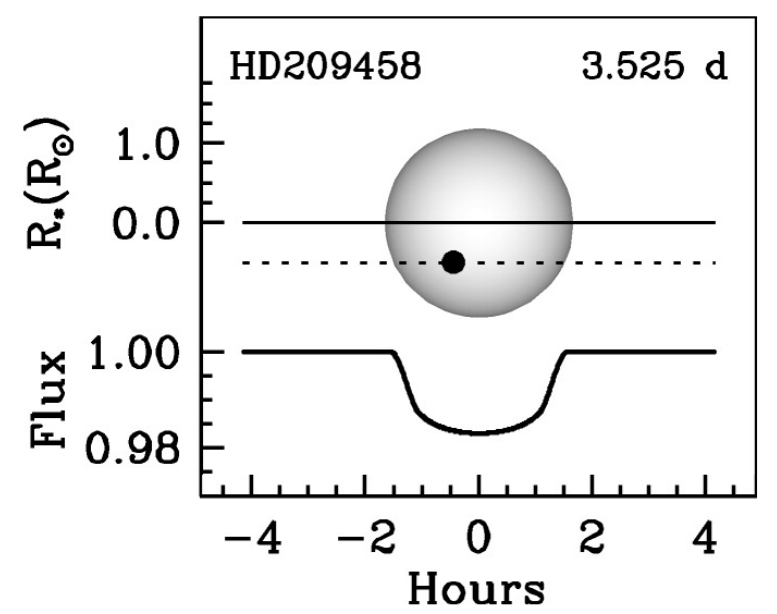

Fig. 1.4. - A best fit optical light curve computed for the parameters of HD 209458b by Torres et al. (2008), with the planet and its host star shown to scale. Transit light curves from HST data are shown in Figure 1.5.

Observations of a few of the HJs in Figure 1.3 have established detections of their upper atmospheres. Most of the observational effort has concentrated on spectroscopy of the optical/infrared continuum, which probes the lower atmosphere at relatively high pressures. A clear example of such transit light curves is given in Figure 1.5 taken from Knutson et al. (2007) for HD 209458b over a bandpass spanning near-ultraviolet (NUV) to near-infrared (NIR) wavelengths. Resonance line spectroscopy of the upper atmosphere has a distinct advantage in that it corresponds to a region larger than the photospheric continuum radius $R_{\mathrm{ph}}$ due to the sharply peaked absorption line cross section. Therefore, most of the progress on the upper atmosphere has come from transmission spectroscopy, which I summarize for the two best studied HJs HD 209458b and HD 189733b. 


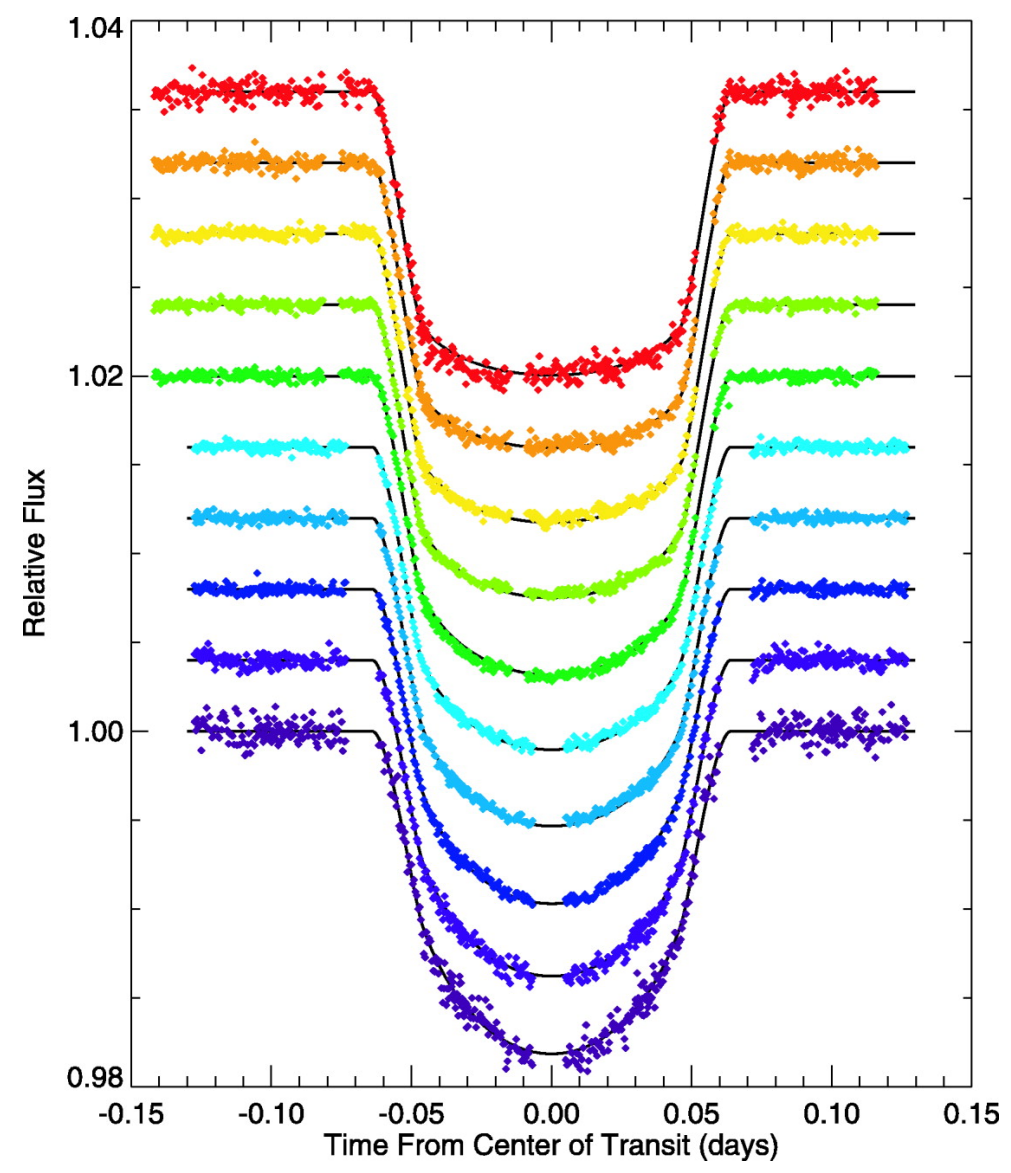

Fig. 1.5.- Normalized in/out of transit flux over a bandpass from $\simeq 300-1000 \mathrm{~nm}$ from HD 209458b from Knutson et al. (2007). The theoretical transit curves are best-fit parameters from all bandpasses (NUV to IR) of HST based on two separate visits. Each transit curve is offset by 0.004 .

\subsubsection{HD 209458b}

Ultraviolet (UV) spectroscopic observations of the hot Jupiter HD 209458b ( $D=$ 0.046 AU; Henry et al. 2000) in transit indicated a 5-10\% increase in absorption in the Lyman $\alpha$ line when compared to out of transit observations, which has been attributed to an inflated atmosphere of neutral H (see Vidal-Madjar et al. 2008, and refs. therein). The size of the optically thick region needed to explain the amount of relative absorption corresponds to an area exceeding the planet's Roche Lobe, which is the region surrounding the planet where its gravitational force dominates that of 
the host star and gas remains bound to the planets atmosphere. Together with the reduction in Lyman $\alpha$ flux at $\approx 100 \mathrm{~km} \mathrm{~s}^{-1}$ from line center, this led Vidal-Madjar et al. (2004) to interpret the transit observations as a signature of atmospheric escape. Other studies have indicated that the exact transit depth can depend on the selected wavelength range of the stellar Lyman $\alpha$ line profile (Vidal-Madjar et al. 2008; BenJaffel 2008), which is likely due to variability in the wings of the stellar Lyman $\alpha$ profile itself. Figure 1.6 compares the observed Lyman $\alpha$ profile for HD 209458b

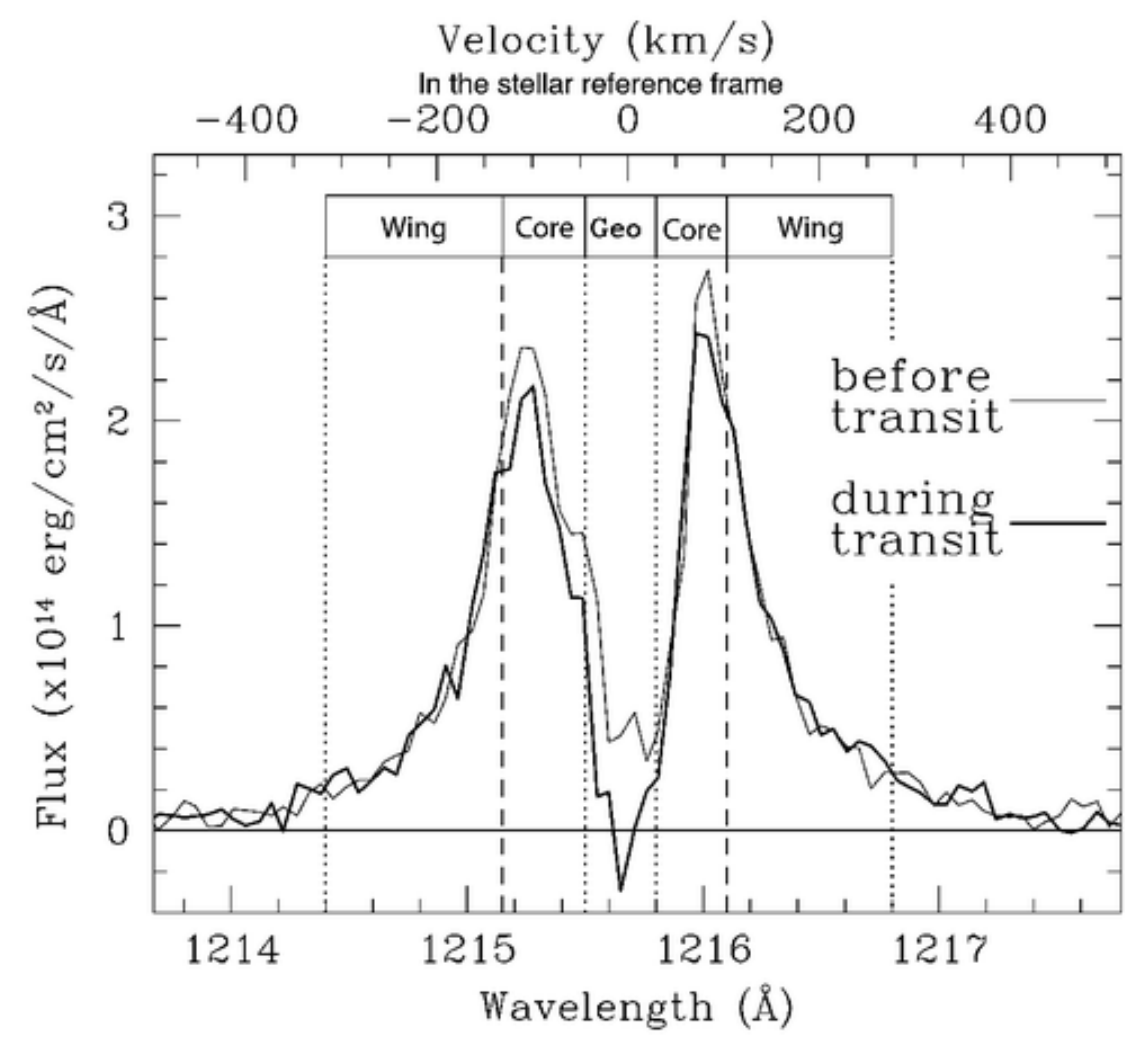

Fig. 1.6. - HD 209458b observed Lyman $\alpha$ profiles before and during planetary transit. The vertical dashed lines indicate the limits of the region where neutral $\mathrm{H}$ absorption by the planetary atmosphere takes place.

(Vidal-Madjar et al. 2008, Figure 1) from before and during transit. The decrease in flux during transit is due to absorption by neutral $\mathrm{H}$ in the planet's atmosphere. The transit depth is an integrated quantity that specifies the total decrease in flux 
over a given bandpass in the stellar Lyman $\alpha$ profile.

Additional transit observations of HD 209458b have indicated absorption in other resonance lines, including NaI (Charbonneau et al. 2002; Sing et al. 2008), OI (VidalMadjar et al. 2004), CII (Vidal-Madjar et al. 2004; France et al. 2011), and SiIII (Linsky et al. 2010; France et al. 2011). Follow-up observations and re-analysis of HST-ACS data in comparison with HST-STIS low and medium-resolution spectra verified the reduction of Lyman $\alpha$ flux (Ehrenreich et al. 2008). Vidal-Madjar et al. (2004) have interpreted the detection of the heavier $\mathrm{O}$ and $\mathrm{C}$ as being entrained in a background $\mathrm{H} / \mathrm{He}$ outflow, which is consistent with some theoretical models (e.g., García Muñoz 2007). Ballester et al. (2007) detected bound-free absorption from $\mathrm{H}$ in the $n=2$ state in transmission spectra for HD 209458b, while ground-based observations have revealed absorption in bound-bound Balmer lines for another HJ called HD 189733b (Jensen et al. 2011), which we discuss in the following section.

\subsubsection{HD 189733b}

Transmission spectra of the hot Jupiter HD 189733b have also revealed absorption due to H (Lecavelier Des Etangs et al. 2010) and NaI (Redfield et al. 2008; Snellen et al. 2008). More recent HST-COS observations by Linsky et al. (2010) have indicated absorption at up to $\pm 50 \mathrm{~km} \mathrm{~s}^{-1}$ from line center in CII and SiIII that may be indicative of high velocity absorbers in the upper atmosphere. Christie et al. (2013) modeled the absorption of stellar $\mathrm{H} \alpha$ as coming from a hydrostatic layer just below the photoionization layer, which can reasonably explain the observed transit depths in $\mathrm{H} \alpha$ and Lyman $\alpha$ for HD 189733b. The extended HI absorption observed for HD 209458b and HD 189733b requires either (1) an extended atmosphere of sufficiently high column, or (2) a thermal velocity of $\sim 100 \mathrm{~km} / \mathrm{s}$ in order to explain the tran- 
sit depths seen in the wings of the Lyman $\alpha$ line far from line center. The current $\mathrm{S} / \mathrm{N}$ and spectral resolution of published data for HD 189733b cannot constrain the detailed density distribution for HI or velocity structure of the upper atmosphere. Multi-epoch spectra have also revealed significant changes in the Lyman $\alpha$ transit depth with time, which has been correlated with flares in ionizing radiation from the host star detected with HST and SWIFT (Lecavelier des Etangs et al. 2012).

In summary, a variety of observations of HD 209458b and HD 189733b are consistent with extended atmospheres of neutral $H$ gas with scale heights $H$ that are a significant fraction of a planetary radius, possibly entraining heavier constituents such as $\mathrm{O}$ and $\mathrm{C}$ in an outflow. Despite all of these detailed observations, it remains unclear what should dominate the expected structure and dynamics of a hot Jupiter upper atmosphere should be. Therefore, what follows is a discussion of the primary components of a more complete model for the upper atmosphere.

\subsubsection{Towards a Model for the Upper Atmosphere}

A model for the upper atmosphere in needed for constraining the density and optical depth distributions as probed by transit observations, as well as for producing a velocity structure, mass-loss rate, and angular momentum loss rate consistent with the data. Previous theoretical models include the direct absorption of EUV photons, which leads to strong heating and an outward temperature increase (i.e., a "thermosphere" analogous to the same high-altitude region of Earth's upper atmosphere). The temperature reaches $\sim 10^{4} \mathrm{~K}$ and can lead to pressure gradients that drive a hydrodynamic (and possible transonic) outflow from the planet.

Since gas giants are mostly $\mathrm{H}$ and $\mathrm{He}$, heating by photoionization of atomic $\mathrm{H}$ dominates. Yelle (2004) and García Muñoz (2007) both computed the detailed structure 
of such a H-dominated atmosphere in photoionization equilibrium. Tian et al. (2005) used a time-dependent hydrodynamical code to compute mass-loss rates from steadystate solutions for a transonic outflow from the upper atmosphere of HD 209458b, as well as for other possible extrasolar planets. Despite that progress that has been made so far, a fundamental oversight of purely hydrodynamical models that attempt to explain the absorption signature is that they have largely ignored the role of the intrinsic planetary magnetic field.

Photoionization leads to an increasing ionization with altitude. The planet's magnetic field will restrict the escape of the products of ionization (electrons and ions), while the escape of neutrals is constrained by drag forces with the confined ions. While a photoionization-driven outflow may be possible in open field line regions, where the ram pressure is sufficient to overcome the net gravitational potential barrier and the magnetic tension forces, such an outflow would also eventually interact with the magnetized stellar wind. Such an interaction could result in the rearrangement of magnetic field lines near the planet, driving charged particles away from the planet's magnetosphere and potentially into the stellar atmosphere (e.g., Cohen et al. 2011).

Unlike the solar system planets, some HJs orbit their host stars inside the stellar Alfvén radius (which, by analogy to the sonic point in a hydrodynamic flow being defined as the location where the flow speed equals the local sound speed), where the accelerating stellar wind speed is equal to the Alfvén speed. Because of the planet's orbit being within the Alfvén radius, readjustments in the star-planet magnetic field may travel upstream back to the star lead to energy dissipation that is observable as enhanced stellar chromospheric activity (Cohen et al. 2011; Shkolnik et al. 2008). In addition, variable stellar chromospheric emission that follows the planet around 
in its orbit (with some phase difference) has been observed with Chandra for the hot Jupiter HD 179949 (e.g., Saar et al. 2008). Although important, the star-planet atmospheric interaction is beyond the scope of this thesis, and my focus will be on the effect of the planetary magnetic field on the upper atmosphere.

The presence of a dynamically important magnetic field is expected to modify the structure of the planetary upper atmosphere significantly. For example, based on magnetohydrodynamical (MHD) theory applied to the stellar wind, Mestel (1968) demonstrated the contrast of the zero magnetic field case of thermally or centrifugally(rotation)-driven outflow, to the magnetically-dominated case. For the magnetized case where both hydrodynamic and magnetic forces are present, outflow ram pressure can overcome magnetic tension forces and magnetic field lines can be combed outward to a more radial configuration. Regions of closed magnetic field lines correspond to regions where the wind is not sufficiently strong to open magnetic field lines.

Within the context of an MHD outflow, the result of a magnetized, hot, rotating surface is the "helmet streamer" configuration for the magnetic field that is a classic feature of MHD winds from an isolated object with a dipole field (see Figure 1.7). The polar region supports an outflow while the equatorial region contains static, magnetically confined gas. But for planets sufficiently close to their parent star, the L1 Lagrange point is inside this dead zone, which results in the possibility that the gas density could increase outward, since the effective gravity points away from the planet. Given the similarity with the magnetized stellar wind problem, this motivates an adaptation of this model to HJ upper atmospheres, which would include the effect of the planet's magnetic field combined with tidal and centrifugal forces. Magnetic field strengths for HJs cannot yet be measured directly, but theoretical work that predicts 


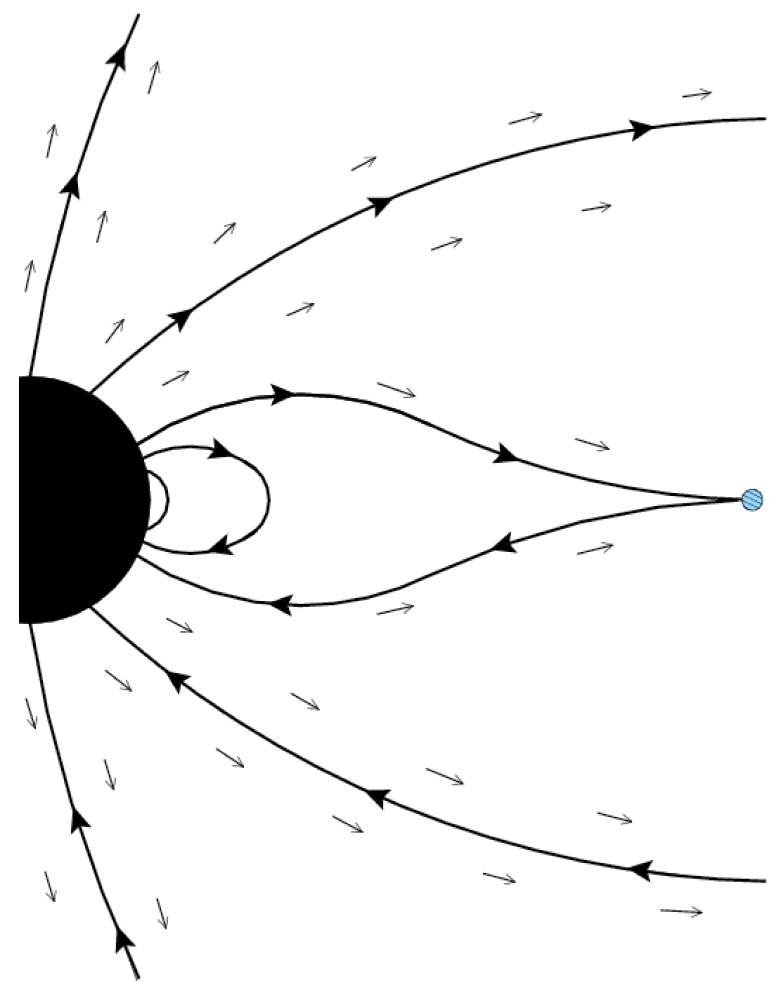

Fig. 1.7.- Schematic model for magnetic field structure (thick lines and arrows) and fluid velocity (thin arrows) resulting from a thermally-driven outflow at the base. In the high-latitude "wind zone", magnetic field lines are open allowing outflow. In the equatorial "dead zone" close to the planet, the gas has zero velocity and no outflow occurs. The dead zone ends in a cusp-type neutral point, denoted by the dashed circle at several planetary radii, outside of which field lines are open at all angles. This figure is characteristic of the weak tide limit, while in the strong tide limit the polar wind would be partially suppressed.

the expected field strengths applicable to highly-irradiated gas giants is summarized in the next section.

\subsubsection{Expected Magnetic Field Strengths}

Earlier work predicts a relatively weak magnetic moment for tidally-synchronized giants (e.g., Sánchez-Lavega 2004), where the field strength associated with the planetary dynamo is assumed to be the result of a balance of Lorentz and Corio- 
lis forces, and thus strongly influenced by rotation rate. However, recent dynamo models, constrained by observations of rapidly-rotating low-mass stars, suggest that field strengths for hot Jupiters may be independent of rotation rate and exceed that of solar system gas giants. For a planetary rotation rate $\Omega$ above a critical value, the field strength $B$ is determined not by $\Omega$ but with core heat flux $F_{\text {core }}$, where dynamo simulations give a scaling $B \sim\left(\rho F_{\text {core }}^{2}\right)^{1 / 3}$ that works well for both planets and low-mass stars (Reiners \& Christensen 2010; Christensen 2009). If core heat fluxes from the deep interiors of hot Jupiters are higher than for Jupiter, as is expected for their larger radii and hotter cores (e.g., Arras \& Bildsten 2006), the expected field strengths may exceed solar system gas giants by more than a factor of 10 .

\subsubsection{Connection to Roche Lobe Overflow Model}

The standard model for mass loss in nearly equal mass binary stars in later stages of stellar evolution involves a donor star filling its own Roche Lobe (i.e., the limiting distance beyond which gas no longer remains bound to the donor star). Any material outside the Roche Lobe will find itself either orbiting the companion or on a circumbinary orbit. A model for mass loss via Roche Lobe overflow in a HJ system would predict a fairly cold, narrow stream of gas through the inner L1 Lagrange point, which is an element of purely hydrodynamic mass loss models (e.g., Lubow \& Shu 1975). A key assumption of the Roche Lobe overflow model is the sonic point $r_{\mathrm{s}, 0} \gg r_{\mathrm{L}}$, where $r_{\mathrm{L}}$ is the size of the planet's Roche Lobe.

A key distinction that limits the applicability of the Roche Lobe overflow model in the context of mass loss from a highly irradiated exoplanet is the unrealistic assumption of a cold, subsonic mass loss through L1. Photoionization heating results in $T \sim 10^{4} \mathrm{~K}$, and the resulting pressure gradients can setup a thermally-driven tran- 
sonic outflow that can have $r_{\mathrm{s}, 0} \sim r_{\mathrm{L}}$. As described in Section 1.2.3, an outflow in the presence of a strong planetary magnetic field can lead to a dead zone of static, magnetically-confined gas, which further limits the applicability of the Roche Lobe overflow model. If MHD effects lead to a dead zone comparable to or even exceeding $r_{\mathrm{L}}$, mass loss through L1 can be quenched by closed magnetic field lines, and mass loss is more complicated than a simplified Roche Lobe overflow model predicts. This realization further motivates the approach to construct an MHD model for mass loss in HJ systems, which is the topic of the following section.

\subsection{Approach \& Methodology}

The observations may be interpreted as gas escaping planets in the form of an outflow driven by a combination of thermal pressure gradients in a heated layer of the upper atmosphere and centrifugal forces from rotation. However, models of the dynamics in their upper atmospheres to date have ignored the role of the intrinsic magnetic field of the planet. The inclusion of magnetic forces, in addition to a full treatment of the non-spherically symmetric tidal forces from the parent star and its application to extrasolar planet atmospheres is not found in the literature.

The preceding sections make arguments for the existence of strong magnetic fields and non-negligible tidal forces from the parent star in HJ systems. Therefore, the combination of thermal, tidal, centrifugal, and magnetic field geometry must all be integrated into a model that predicts the resulting mass and angular momentum loss from the planet. The significance of this thesis is that it presents, for the first time, both analytic work and numerical simulations of HJ upper atmospheres shaped by an expected magnetic field and stellar tides. The resulting density profiles, wind and magnetic field strength and structure in steady-state, as well as their dependence on 
the stellar tide and conditions at the base of the outflow, can be applied to make contact with the observational results from transiting HJs within the database of exoplanets.

In order to accomplish these goals, the thesis is organized as follows. Chapter 2 connects previous work in the literature on stellar winds to construct a semi-analytic model for the magnetically-dominated upper atmospheres of HJs and a comparison to observations of HD 209458b at transit. Chapter 3 summarizes the MHD code ZEUSMP, which is used to generalize the MHD model that is presented in Chapter 4 by relaxing the assumption of a magnetic field geometry and allowing the competition between wind and magnetic forces to achieve steady-state solutions. The resulting transit profiles are compared with observations of HD 209458b's and HD 189733b's upper atmosphere to demonstrate the validity of the MHD model results. A summary of the main findings of the thesis and suggestions for future work on the model are in Chapter 5. 


\section{Chapter 2}

\section{An MHD Model of the Upper}

\section{Atmosphere}

\subsection{Overview}

We model the magnetized, hot, rotating portion of the planet's upper atmosphere in the context of magnetohydrodynamic (MHD) outflows, the theory of which was originally developed for stars and accretion disks. Figure 1.7 in Chapter 1 is an illustration of the expected structure for the wind from an isolated object with a surface dipole field: the polar region supports an outflow since $T \sim 10^{4} \mathrm{~K}$ due to EUV flux from the parent star (Yelle 2004), while the equatorial region contains static, magnetically confined gas. This configuration applies interior to the magnetospherestellar wind interaction. Tidal forces are an important consideration, and we will show that tides may strongly affect the density profile and size of the static region, and may even shut the wind off in the polar region.

The first half of this chapter ( $§ 2.2$ through $\S 2.7$ ), develops a general theory of isothermal hot Jupiter (HJ) magnetospheres. Section 2.2 discusses the problem setup 
and approximations used to obtain a solution. Planetary magnetic field strengths predicted by dynamo models are discussed in more detail in section 2.3. Section 2.4 discusses qualitative features of atmospheric structure, and motivation for the existence of a dead zone. Section 2.5 reviews the centrifugal and tidal forces, and discusses the projection of these forces along magnetic field lines. The structure of the dead zone is discussed in Section 2.6, followed by the wind zone in Section 2.7.

The second half of the chapter ( $\S 2.8$ through $\S 2.12)$ uses the computed Lyman $\alpha$ transmission spectra to constrain parameters of the global 3D models. In Section 2.8, we construct a 1D model in hydrostatic, thermal, and ionization balance to compute appropriate values for the base pressure and temperature for the 3D global models that we present in Section 2.9. Mass loss rates are computed and spin-down torques are estimated, in Section 2.10. Neutral H column density maps for the global models are used to illustrate the dependence on key parameters in Section 2.11. Lyman $\alpha$ transmission spectra in comparison with observations and scattering of stellar Lyman $\alpha$ by the planet are presented in Section 2.12. Finally, we compare and contrast our magnetic wind model with the standard Roche Lobe overflow model in Section 2.13. The MHD wind equations and ion-neutral coupling are discussed in Appendices 2.15 and 2.16 , respectively.

The following results were accepted and published by the Astrophysical Journal, Volume 728, Page 152 (hereafter Trammell et al. 2011).

\subsection{Problem Setup \& Approximations}

In this section we outline the problem to be solved, and the simplifying assumptions used to find solutions. Except for section 2.8, this paper discusses a 3D isothermal model of the upper atmosphere. The isothermal model is parametrized by an effective 
sound speed, as well as the pressure at a fiducial radius. Appropriate values for these parameters are discussed in a simple 1D spherical model in section 2.8, including photoionization and thermal equilibrium.

In the 3D model, we compute approximate solutions of the one-fluid MHD equations with an inner boundary at the base of the warm $\mathrm{H}$ layer, and an outer boundary which extends to at least ten planetary radii (or one stellar radius). We treat the gas as having constant isothermal sound speed $a=\sqrt{k_{b} T / \mu m_{p}}$, where $T$ is gas temperature of the fluid, $\mu$ is the mean molecular weight, $k_{b}$ is Boltzmann's constant, and $m_{p}$ is the proton mass. At the inner boundary, the density and pressure are assumed to follow equipotentials. We assume the planet's rotation rate is synchronized with its orbital motion around the parent star, giving orbital and spin angular velocity $\Omega$. We work in a coordinate system centered on the planet and rotating at rate $\Omega$. The stellar gravity is included in the tidal approximation, and an effective potential $U$ is composed of the planetary gravity, stellar gravity, and the centrifugal force. We specify a specific magnetic field geometry which is dipole near the planet and matches onto a radial field at the dead zone radius. We compute the ionization fraction with a simple, optically thin model applied to the derived gas densities of the MHD model. To summarize, we have made several simplifying assumptions in order to focus on the new physics arising from MHD effects. We now discuss these simplifying approximations in more detail.

The simultaneous inclusion of photoionization heating, chemical reactions and collisional coupling between different species, stellar tidal forces, and the simultaneous interaction with the stellar wind in the presence of magnetic field is a formidable problem. Our approach is to first ignore the interaction with the stellar wind, but to include the effect of the stellar tidal forces felt by the planet's atmosphere. The 
interaction with the stellar wind may alter the results of this paper in several ways (see e.g., Murray-Clay et al. 2009; Stone \& Proga 2009). The stellar wind will limit the size of the magnetosphere, as determined by stress balance at the magnetopause (e.g., Preusse et al. 2007). Reconnection between field lines in the stellar wind and magnetosphere may lead to magnetospheric convection, limiting the high density region to be inside a plasmapause (Schunk \& Nagy 2004), as for Earth. Finally, reconnection may also generate non-thermal plasma populations. We do not consider the interaction with the stellar wind in order to construct the simplest possible model.

Another key approximation is that we treat photoionization heating as being spherically symmetric, creating a hot layer uniformly over the planet. In reality, the night side temperature and ionization state may depend on day-night heat redistribution, and downward heat conduction along field lines not in the planet's shadow. In perfect MHD, such redistribution would be highly constrained in the magnetically dominated upper atmosphere, but finite conductivity may allow field lines to slip through the gas (Gold 1959). Even on the day side, large gas density outside the Roche lobe may project a non-spherically symmetric shadow on the deeper layers.

Near the planet, the dynamo-generated, roughly dipole field from the planets core is expected to dominate. Moving outward, currents generated in the magnetosphere comb the field lines into a nearly radial direction beyond the dead zone radius. Such a geometry has been used before in the context of the stellar wind (Mestel 1968; Mestel \& Spruit 1987; Okamoto 1974) and we will adopt it here. This field geometry will be implemented in the global models presented in section 2.9, and is motivated in the discussion of Appendix 2.16.

Finally, the one-fluid approximation assumes that mean free paths are sufficiently small that relative motion of different species can be ignored. In Appendix 2.16 
we will check this assumption a posteriori for our models, which estimate particle densities and velocities in the dead and wind zones. Specifically, we will show that the electron-proton-hydrogen atom gas is well coupled collisionally for the parameters of interest, and therefore the drift velocity is small and hydrogen atoms have short mean free paths in the dead zone region. As a consequence, neutral hydrogen atoms do not fly ballistically through the magnetosphere, and photoionization equilibrium is a good approximation when computing the ionization fraction. Further, we compute the rate at which magnetic field can drift relative to the fluid. For this thermal population of particles in the magnetosphere, we find that the dead zone gives the largest observable transit signal, and that the bulk of the hydrogen atoms in the dead zone are not escaping.

In the next section we will review expected magnetic field strengths for hot Jupiters.

\subsection{Magnetic Field Strengths}

The importance of the magnetic field for the upper atmosphere depends critically on the field strength. However, the magnetic fields of hot Jupiters are currently unconstrained by observation. This section will use theoretical considerations to estimate likely field strengths for hot Jupiters.

Sánchez-Lavega (2004) computed that Rayleigh numbers in hot Jupiters are typically much larger than the critical Rayleigh number for thermal convection in the metallic core. Using estimates of the fluid velocity carrying the heat flux, he found that the magnetic Reynolds number is much larger than unity, and that dynamo action can occur. He argued that if the dynamo operated with Elsasser number of order unity, then $B \sim\left(2 \rho \Omega \lambda_{B}\right)^{1 / 2}$, where $\rho$ is the mass density and $\lambda_{B}$ is the magnetic 
diffusivity. The dominant scaling important for hot Jupiters is then with rotation: $B \propto \Omega^{1 / 2}$. For synchronized planets with orbital periods of a few days, this scaling predicts that the field for hot Jupiters should be smaller than that of Jupiter (equatorial field $B_{\mathrm{J}, \mathrm{eq}}=4.3 \mathrm{G}$ ) by a factor of a few.

The opposite conclusion may be drawn from the recent results of Christensen et al. (2009). As the rotation rate is increased above a critical value, the field strength no longer increases with rotation rate, and dynamo simulations give a magnetic field strength $B \sim\left(\rho F_{\text {core }}^{2}\right)^{1 / 3}$, nearly independent of rotation rate and magnetic diffusivity, where $F_{\text {core }}$ is the heat flux escaping from the conducting core. Christensen et al. (2009) show that this scaling applies to both planets and rapidly rotating, low mass stars over many orders of magnitude in heat flux. They argue that the dependence on $F_{\text {core }}$ arises since it is the heat flux reservoir that sustains the magnetic field against Ohmic dissipation. To be in the saturated regime, the Rossby number Ro must satisfy $R o=V_{\text {ed }} / \Omega \ell \lesssim 0.1$, where $V_{\text {ed }}$ is the typical velocity of the eddies transporting heat, and $\ell$ is the size of the conducting region; $\ell \sim R$ for $M_{p} \gtrsim 0.5 M_{J}$. Sánchez-Lavega (2004) estimated synchronized planets in few day orbits to have $R o \ll 0.1$, using heat fluxes comparable to that of Jupiter. Hence these planets are expected to be in the saturated regime.

The large radii of hot Jupiters are currently not well understood, since the cooling and contraction time for passively cooling planets, even allowing for irradiation by the star, is far shorter than the age for a number of observed planets (e.g., Fortney \& Nettelmann 2009). This has led to the suggestion that these planets are not passively cooling, but rather have an anomalous source of internal heating, which is as yet unidentified but balances the core cooling rate. To assess the required heating rates, Arras \& Socrates (2009) computed cooling flux from the core for planets as a function 
of radius (their Figure 11; see Arras \& Bildsten 2006, for a discussion of the cooling luminosity of irradiated hot Jupiters). For Jupiter-mass planets in the radius range $R_{\mathrm{ph}}=1.3-1.5 R_{J}$, the cooling flux is larger than that of Jupiter by a factor $10^{2}-10^{3}$, for which the Christensen et al. (2009) scaling would give magnetic fields 5-10 times larger than Jupiter. Larger mass planets with the same radius would have larger cooling fluxes, and vice versa. For instance, WASP 12b, WASP 17b and TRES 4 have radii $R_{\mathrm{ph}} \sim 1.8 R_{J}$ and masses in the range $0.5-1.5 M_{J}{ }^{1}$, for which the cooling fluxes would be $10^{3}-10^{4}$ times higher than Jupiter, implying fields larger than Jupiter by factors of $10-20$.

In summary, the recent results on dynamo theory from Christensen et al. (2009), and the assumption that hot Jupiter cores are subject to an externally powered heating (Arras \& Socrates 2009), argue that field strengths may be up to an order of magnitude larger than that of Jupiter. We will take this as motivation to explore a wide range of parameter space for the magnetic field in our calculations.

In the next section, we show that the photoionized $\mathrm{H}$ and $\mathrm{H}^{+}$layers are magnetically dominated for field strengths comparable to Jupiter or Saturn, and motivate the existence of a dead zone by a toy problem.

\subsection{Dead-Zone/Wind-Zone Structure of the Up- per Atmosphere}

Yelle (2004) and García Muñoz (2007) presented detailed calculations of the transition between the molecular lower atmosphere $\left(\mathrm{H}_{2}\right)$, the layer dominated by atomic hydrogen $(\mathrm{H})$, and the ionized upper atmosphere $\left(\mathrm{H}^{+}\right)$. In this paper, we restrict

\footnotetext{
${ }^{1}$ http://exoplanet.eu/catalog-transit.php
} 
attention to the $\mathrm{H}$ and $\mathrm{H}^{+}$regions, where the transmission spectrum is formed. The strong heating in these layers due to UV photon energy deposition raises the temperature to $T \simeq 10^{4} \mathrm{~K}$. As a consequence of the increased temperature and low mean molecular weight, the radial extent of the $\mathrm{H}$ and $\mathrm{H}^{+}$layers $\left(\gtrsim R_{J}\right)$ is expected to be much larger than that of the $\mathrm{H}_{2}$ layer above the photosphere $\left(\lesssim(0.1-0.2) \times R_{J}\right)$.

We define the lower boundary of our wind model to be at the base of the warm ( $T \gtrsim 5000 \mathrm{~K}) \mathrm{H}$ layer, at base radius $R$ and base pressure $P_{\text {base }}$. The base of the warm layer is a crucial parameter for the transit depth. As discussed in the phenomenological model of Koskinen et al. (2010), the transit depth of HD 209458b could be understood as being due to thermal $\simeq 10^{4} \mathrm{~K} \mathrm{H}$ gas extending down to $\sim 10-100 \mathrm{nbar}$ pressures. In section 2.8 , we compute a simple $1 \mathrm{D}$ model for ionization and thermal equilibria in the $\mathrm{H}$ and $\mathrm{H}^{+}$layers which shows that such base conditions are indeed possible.

The Lyman $\alpha$ transmission spectrum of HD 209458b shows absorption by the planetary atmosphere at linewidths $\Delta v \gtrsim 100 \mathrm{~km} \mathrm{~s}^{-1}$ from line center. At this linewidth, the cross section is $\sigma_{\nu} \simeq 2 \times 10^{-19} \mathrm{~cm}^{2}$ (see Figure 2.14). Optical depth unity requires a hydrogen column $N_{H} \simeq 1 / \sigma_{\nu} \simeq 5 \times 10^{18} \mathrm{~cm}^{-2}$. Assuming the gas is dominated by atomic hydrogen, the pressure at this level in the atmosphere is $P \simeq g m_{p} N_{H} \simeq 2 \operatorname{nbar}\left(g / 300 \mathrm{~cm} \mathrm{~s}^{-2}\right)$ (see Figure 2.9). This is a factor $\sim 10^{8}$ more rarefied than the optical photosphere for continuum radiation at pressure $P_{\mathrm{ph}} \simeq$ 100 mbar. Magnetic forces dominate in this layer if $B^{2} / 8 \pi \gtrsim 2 \mathrm{nbar}\left(\mathrm{g} / 300 \mathrm{~cm} \mathrm{~s}^{-2}\right)$, implying a critical field strength $B_{\text {crit }} \gtrsim 0.25 \mathrm{G}\left(\mathrm{g} / 300 \mathrm{~cm}^{2} \mathrm{~s}^{-1}\right)^{1 / 2}$. This is less than Jupiter's equatorial magnetic field $B_{\mathrm{J}, \mathrm{eq}}=4.3 \mathrm{G}$ and comparable to Saturn's equatorial field $B_{\mathrm{S} \text {,eq }}=0.22 \mathrm{G}$. Moving upward, if the gas pressure drops much faster than magnetic pressure, the atmosphere can become highly magnetically dominated 
— a magnetosphere.

The theory of thermally and magneto-centrifugally driven MHD winds gives guidance on the upper atmosphere structure in the magnetically dominated case (for a good review see Spruit 1996). Consider a thought experiment in which a nonmagnetic spherically symmetric wind with velocity $v_{\infty}$ and mass loss rate $\dot{M}$ exists at time $t<0$, and at time $t=0$ a dipole magnetic field is turned on. On which field lines can the wind overpower the magnetic forces and open the field lines to infinity? The magnetic pressure on the equator $(\theta=\pi / 2)$ is weaker than at the footpoint (at angle $\theta_{b}$ ) by a factor $\left[B(\pi / 2) / B\left(\theta_{b}\right)\right]^{2}=[R / r(\pi / 2)]^{6}=\sin ^{12} \theta_{b}$ (see Eq. 2.12). This powerful dependence on footpoint position means there are always field lines near the magnetic poles which open to infinity on which a wind can outflow (see Figure 1.7 for a cartoon). The reason is that at the equator, the wind ram pressure can overcome the steeply falling magnetic pressure at sufficiently large distance from the planet. If we assume the wind ram pressure decreases outward as $\rho v^{2}=\dot{M} v_{\infty} / 4 \pi r^{2}$, then the critical footpoint angle inside of which a "polar wind" occurs is $\sin \theta_{b} \simeq\left(\dot{M} v_{\infty} / R^{2} B_{0}^{2}\right)^{1 / 8}$. For fiducial polar magnetic field $B_{0}=8.6 \mathrm{G}$, $R=1.4 R_{J}$, (constant) flow speed $v_{\infty}=10 \mathrm{~km} \mathrm{~s}^{-1}$ and mass loss rate $\dot{M}=10^{11} \mathrm{~g} \mathrm{~s}^{-1}$ (motivated by the studies of Yelle 2004; García Muñoz 2007; Murray-Clay et al. 2009), we find the polar cap size occupied by open field lines is $\theta_{b} \simeq 14^{\circ}$, and the last closed field lines is at equatorial radius $r(\pi / 2) \simeq 16 R$. This static, closed field line region is referred to as the "dead zone" (Mestel 1968). These estimates suggest that the region within a few planetary radii, where the transit signal arises, is filled primarily with static gas in the dead zone, rather than outflowing gas, as has been previously assumed.

For close-in, tidally-locked planets, tides and centrifugal forces play a key role in 
upper atmosphere structure. In the next section we review the effective potential near the planet, and the projection of forces along dipole field lines.

\subsection{Tidal Forces \& Magnetic Geometry}

Gas in the upper atmosphere of the planet is subject to three potential forces: gravity from the planet, tidal gravity from the star, and the centrifugal force due to the planetary rotation. For a synchronized planet, the centrifugal and tidal forces, or just tidal force for short, are comparable in strength, although their angular dependence is different. For position vector $\boldsymbol{x}=(r, \theta, \phi)$ relative to the center of the planet, and star at position $\boldsymbol{x}_{\star}=(D, \pi / 2,0)=D \boldsymbol{e}_{x}$, the sum of the three accelerations is

$$
\boldsymbol{a}(\boldsymbol{x})=-\nabla U(\boldsymbol{x}),
$$

where the effective potential is given by

$$
\begin{aligned}
U(\boldsymbol{x}) & =-\frac{G M_{p}}{|\boldsymbol{x}|}-\frac{G M_{\star}}{\left|\boldsymbol{x}-\boldsymbol{x}_{\star}\right|}+\frac{G M_{\star} \boldsymbol{x} \cdot \boldsymbol{x}_{\star}}{\left|\boldsymbol{x}_{\star}\right|^{3}}-\frac{1}{2}|\boldsymbol{\Omega} \times \boldsymbol{x}|^{2} \\
& \simeq-\frac{G M_{p}}{r}-\frac{1}{2} \Omega^{2} r^{2}\left(f \sin ^{2} \theta-1\right) .
\end{aligned}
$$

Equipotentials are shown in Figure 6.2 of Kopal (1978). The longitude-dependent function $f=1+3 \cos ^{2} \phi$. The vector angular velocity of the orbit is $\boldsymbol{\Omega}=\Omega \boldsymbol{e}_{z}$, where $\boldsymbol{e}_{z}$ is normal to the orbital plane, and $\Omega=\left[G\left(M_{\star}+M_{p}\right) / D^{3}\right]^{1 / 2}$. The first and second terms in Eq. 2.2 are the potential of the planet and star, respectively. The third term in Eq. 2.2 is due to the motion of the origin of the coordinate system. The last term in Eq. 2.2 is due to the centrifugal force. It may be shown that Eq. 2.2 is equivalent to the usual Roche potential with origin at the center of mass by combining the third 
and fourth terms. The form in Eq. 4.10 is an expansion in the limit $r \ll D$, and agrees with that for "Hill's limit" found in Murray \& Dermott (2000) when evaluated in the orbital plane $(\theta=\pi / 2)$.

The accelerations are given by

$$
\begin{aligned}
a_{r} & =-\frac{\partial U}{\partial r}=-\frac{G M_{p}}{r^{2}}+\Omega^{2} r\left(f \sin ^{2} \theta-1\right) \\
a_{\theta} & =-\frac{1}{r} \frac{\partial U}{\partial \theta}=f \Omega^{2} r \sin \theta \cos \theta \\
a_{\phi} & =-\frac{1}{r \sin \theta} \frac{\partial U}{\partial \phi}=-3 \Omega^{2} r \sin \theta \sin \phi \cos \phi
\end{aligned}
$$

The vector acceleration $\boldsymbol{a}$ should not be confused with the sound speed $a$. If we denote the coordinate along the star-planet line $x=r \sin \theta \cos \phi$, and normal to the orbit plane $z=r \cos \theta$, then the tidal force is outward when $3 x^{2}>z^{2}$, i.e. within latitudes $-\pi / 3$ to $\pi / 3$ of the equator. The tidal force is zero in the $y$-direction. Hence when observing a planet in the plane of the sky during transit, the tidal forces are inward along $\boldsymbol{e}_{z}$, zero along $\boldsymbol{e}_{y}$, and away from the planet along $\boldsymbol{e}_{x}$, the line of sight during transit.

The L1 and L2 Lagrangian points at radii $r_{\mathrm{L} 1}$ and $r_{\mathrm{L} 2}$ are found by using Eq. 2.4 along the star-planet line $(\theta=\pi / 2, \phi=0)$, giving

$$
r_{\mathrm{L} 1} \simeq r_{\mathrm{L} 2} \simeq r_{\mathrm{L}} \equiv D\left(M_{p} / 3 M_{\star}\right)^{1 / 3}=\left(G M_{p} / 3 \Omega^{2}\right)^{1 / 3}
$$

The near equality $r_{\mathrm{L} 1} \simeq r_{\mathrm{L} 2}$ is due to $M_{\mathrm{p}} / M_{\star} \ll 1$ in Eq. 2.7. The photospheres of the observed planets are inside the L1-L2 radii.

How does the magnetic field alter the radius beyond which the net gravity points outward? What is needed is the projection $a_{\|}=\boldsymbol{a} \cdot \boldsymbol{b}$ along field lines, where $\boldsymbol{b}=\boldsymbol{B} / B$ is the unit vector along the magnetic field direction. In dipole geometry, approxi- 
mately correct near the planet,

$$
\boldsymbol{B}=B_{0}\left(\frac{R}{r}\right)^{3}\left(\boldsymbol{e}_{r} \cos \theta+\boldsymbol{e}_{\theta} \frac{\sin \theta}{2}\right)
$$

and the unit vector is

$$
\boldsymbol{b}=\frac{1}{N}\left(\boldsymbol{e}_{r} \cos \theta+\boldsymbol{e}_{\theta} \frac{\sin \theta}{2}\right)
$$

where the normalization factor is

$$
N=\sqrt{\cos ^{2} \theta+\sin ^{2} \theta / 4}=\sqrt{1-3 \sin ^{2} \theta / 4}
$$

The parallel acceleration is then

$$
a_{\|}=-\boldsymbol{b} \cdot \nabla U=\frac{\cos \theta}{N}\left[-\left(\frac{G M_{p}}{r^{2}}+\Omega^{2} r\right)+\frac{3}{2} f \Omega^{2} r \sin ^{2} \theta\right] .
$$

The quantity in brackets in Eq. 2.11 must be positive in order for the net acceleration to be away from the planet. To solve for the radius at which the net acceleration is zero, we express $\theta$ in terms of $r$ along dipole field lines using

$$
r(\theta)=R \frac{\sin ^{2} \theta}{\sin ^{2} \theta_{b}} \equiv r_{\mathrm{eq}} \sin ^{2} \theta
$$

where

$$
r_{\mathrm{eq}}=R / \sin ^{2} \theta_{b}
$$

is the equatorial radius of the field line with footpoint at $\theta_{b}$. The "magnetic Roche lobe radius", $r_{\mathrm{RB}}$, at which the projected acceleration $a_{\|}=0$ is given by the solution 
of the equation

$$
\frac{G M_{p}}{r_{\mathrm{RB}}^{2}}+\Omega^{2} r_{\mathrm{RB}}=\frac{3}{2} f \Omega^{2} \frac{r_{\mathrm{RB}}^{2}}{r_{\mathrm{eq}}} .
$$

Solutions can only exist in the range of radii $2 r_{\text {eq }} / 3 f \leq r_{\mathrm{RB}} \leq r_{\text {eq }}$. Solutions for $r_{\mathrm{RB}}$ exist first at the looptop $r_{\mathrm{RB}}=r_{\text {eq }}$ for loops of critical size

$$
r_{\text {eq,crit }}=\frac{r_{\mathrm{L}}}{(f / 2-1 / 3)^{1 / 3}}
$$

That is, when the loop size becomes larger than about the L1-L2 distance, the outer part of the loop can have net acceleration pointing away from the planet. Note that this statement applies even in the plane where $\cos ^{2} \phi=0$, where the radial component of the tidal force points inward. The magnetic geometry allows a "magnetic Roche lobe" $r_{\mathrm{RB}} \sim r_{\mathrm{R}}$ to exist at all longitudes, in contrast to the unmagnetized case.

In the next section we model the gas density in the dead zone.

\subsection{The Dead Zone}

The 3D MHD wind equations are presented in Appendix 2.15. There we derive the Bernoulli constant along field lines and discuss how gas pressure discontinuities at the dead zone - wind zone boundaries give rise to current sheets which alter the magnetic field configuration. For the present section which discusses the dead zone, the main concept needed is that hydrostatic balance applies along field lines. We will approximate the field lines as dipolar.

In the dead zone, the velocity along field lines $\boldsymbol{v}=0$. Setting $\boldsymbol{v}=0$ in Eq. 2.71 and dotting this equation with $\boldsymbol{b}$ to eliminate the Lorentz force we find the equation 
of hydrostatic balance along field lines

$$
\frac{1}{\rho} \frac{d P}{d s}=a^{2} \frac{d \ln \rho}{d s}=-\frac{d U}{d s}
$$

where $d / d s=\boldsymbol{b} \cdot \boldsymbol{\nabla}$ is the derivative along field lines. Under the isothermal assumption, Eq. 4.5 can then be integrated to give the run of pressure and density along a field line with base position $\left(\theta_{b}, \phi\right)$ :

$$
\begin{aligned}
\frac{P(r, \theta, \phi)}{P\left(R, \theta_{b}, \phi\right)} & =\frac{\rho(r, \theta, \phi)}{\rho\left(R, \theta_{b}, \phi\right)} \\
& =\exp \left[-\left(\frac{U(r, \theta, \phi)-U\left(R, \theta_{b}, \phi\right)}{a^{2}}\right)\right]
\end{aligned}
$$

We treat the density and pressure at the base as being along equipotentials. Defining $\rho_{s s}=P_{s s} / a^{2}=\rho(r=R, \theta=\pi / 2, \phi=0)$ to be the value at the substellar point at the base radius, the density at the base radius at other points is

$$
\rho_{b}\left(\theta_{b}, \phi\right)=\rho_{s s} \exp \left[-\left(\frac{U\left(R, \theta_{b}, \phi\right)-U(R, \pi / 2,0)}{a^{2}}\right)\right]
$$

Combining Eq. 2.17 and 2.18 then gives

$$
\begin{aligned}
\frac{P(r, \theta, \phi)}{P_{s s}} & =\frac{\rho(r, \theta, \phi)}{\rho_{s s}} \\
& =\exp \left[-\left(\frac{U(r, \theta, \phi)-U(R, \pi / 2,0)}{a^{2}}\right)\right]
\end{aligned}
$$

Note that because we have assumed the density and pressure surfaces at the base are along equipotentials, Eq. 2.19 satisfies

$$
0=-\nabla P-\rho \nabla U
$$


in all three directions, not just along field lines. In this case $-\nabla U$ is balanced by gas pressure forces due to a non-spherical distribution of mass. If, on the other hand, temperature, density or pressure surfaces were not along equipotentials, then Eq. 2.20 would not be satisfied perpendicular to field lines, and the trans-field force balance (Eq. 2.81) would be required to understand the required currents.

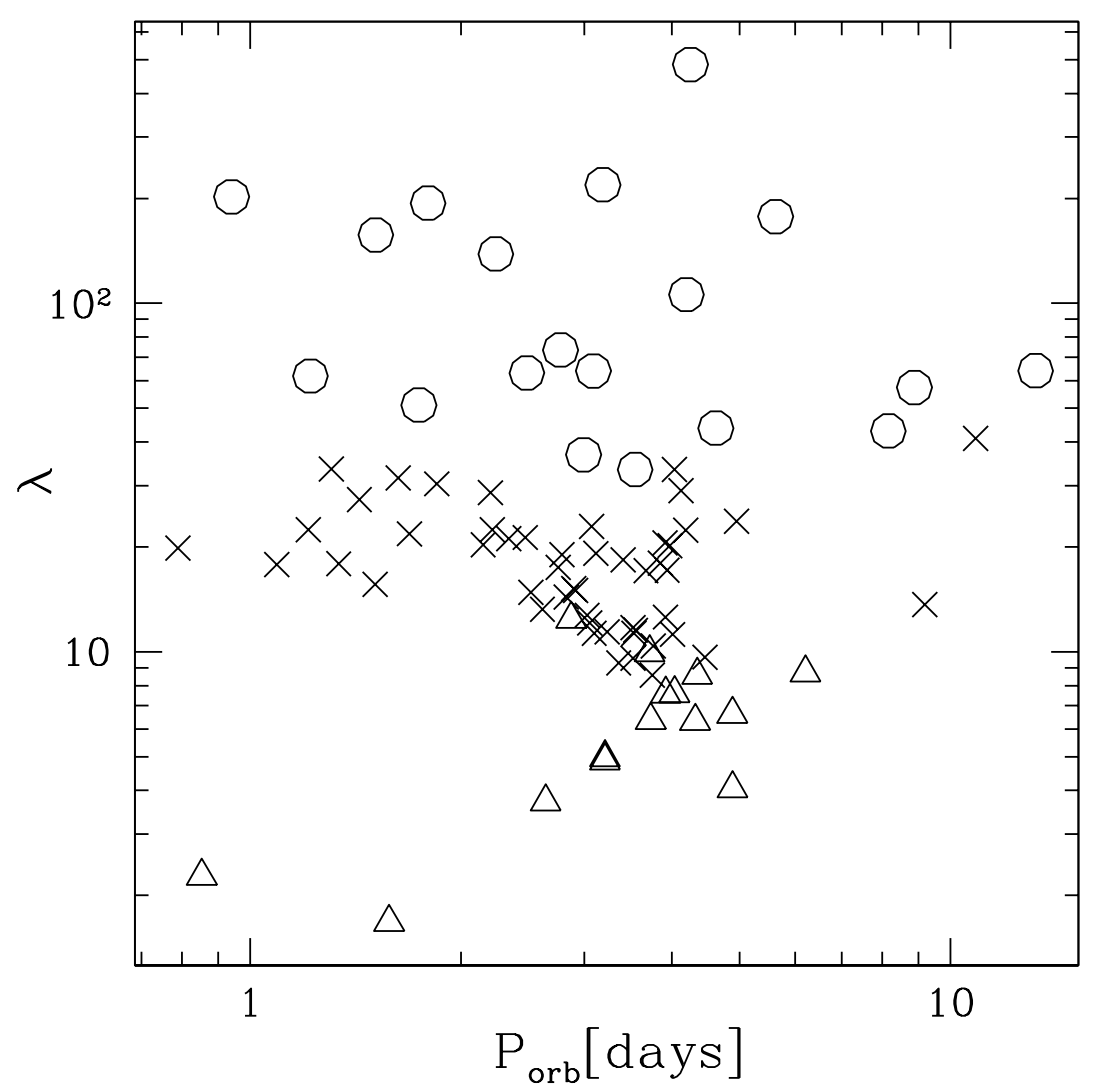

Fig. 2.1.- Values of $\lambda$ calculated using Eq. 2.22 for the current database of transiting exoplanets. The parameters used are $a=9.3 \mathrm{~km} \mathrm{~s}^{-1}$, appropriate to $T=10^{4} \mathrm{~K}, \mu=$ 1 , and $R=R_{\mathrm{ph}}$. The open circles, X's and open triangles are planets with $M_{p} / M_{J}>$ $2,1 / 2 \leq M_{p} / M_{J} \leq 2$ and $M_{p} / M_{J}<1 / 2$, respectively. Values of $P_{\mathrm{orb}}, M_{p}$ and $R_{\mathrm{ph}}$ are taken from the current database of transiting exoplanets (http://exoplanet.eu/). 
The potential difference in Eq. 2.19 can be written in dimensionless form

$$
\begin{aligned}
& \frac{U(r, \theta, \phi)-U(R, \pi / 2,0)}{a^{2}} \\
&=\lambda\left(1-\frac{R}{r}\right)+\frac{1}{2} \epsilon\left[3-\left(\frac{r}{R}\right)^{2}\left(f \sin ^{2} \theta-1\right)\right]
\end{aligned}
$$

where we have defined the ratio of escape to thermal speed

$$
\begin{aligned}
\lambda & =\frac{G M_{p}}{R a^{2}} \\
& \simeq 9.3\left(\frac{M_{p}}{0.7 M_{J}}\right)\left(\frac{1.4 R_{J}}{R}\right)\left(\frac{10 \mathrm{~km} \mathrm{~s}^{-1}}{a}\right)^{2}
\end{aligned}
$$

and the ratio of rotation speed (or tidal potential) to thermal speed

$$
\begin{aligned}
\epsilon & =\left(\frac{\Omega R}{a}\right)^{2} \\
& =0.043\left(\frac{3.5 \text { days }}{P_{\text {orb }}}\right)^{2}\left(\frac{R}{1.4 R_{J}}\right)^{2}\left(\frac{10 \mathrm{~km} \mathrm{~s}^{-1}}{a}\right)^{2}
\end{aligned}
$$

The photoionization model in section 2.8 shows outward increase in $T$ and decrease in $\mu$, implying larger $a$ with radius. The density will then decrease more slowly than in the isothermal model for the same quantities at the base.

Figures 2.1 and 2.2 show the values of $\lambda$ and $\epsilon$ versus planet orbital period for the transiting exoplanets, taking $M_{p}$, transit radius $R_{\text {ph }}$ and orbital period $P_{\text {orb }}=2 \pi / \Omega$ from the Extrasolar Planets Encyclopedia ${ }^{2}$. The temperature and mean molecular weight have been set to fiducial values of $\mu=1$ and temperature $T=10^{4} \mathrm{~K}$, giving sound speed of $a=9.3 \mathrm{~km} \mathrm{~s}^{-1}$.

Note that a substantial number of planets have $\lambda=2-10$, implying the scale height of the gas is large enough that the density decrease in the dead zone is only

\footnotetext{
${ }^{2}$ http://exoplanet.eu/
} 


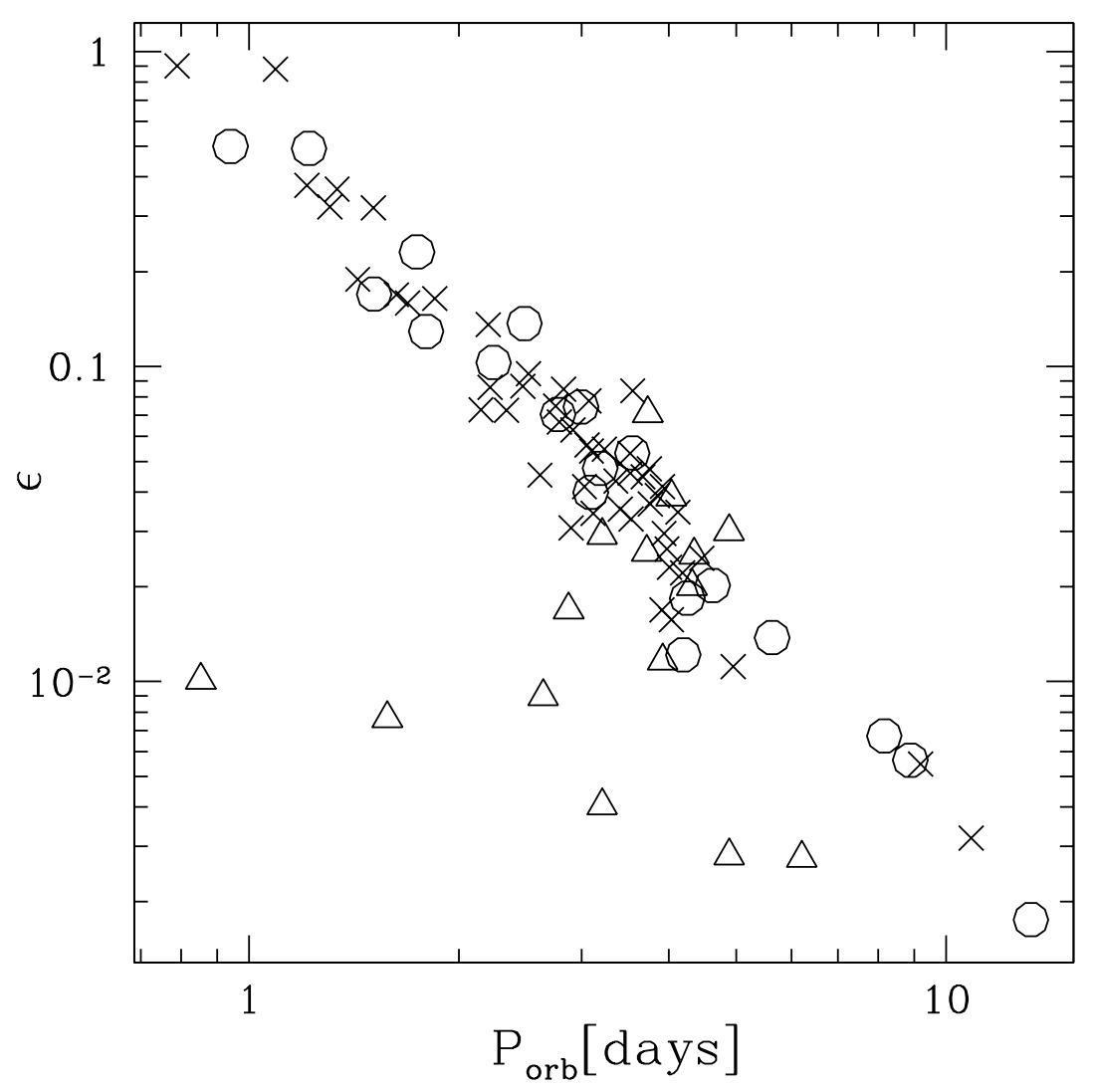

Fig. 2.2.- Values of $\epsilon$ calculated using Eq. 2.23 for the current database of transiting exoplanets (http://exoplanet.eu/). The open circles, X's and open triangles are planets with $M_{p} / M_{J}>2,1 / 2 \leq M_{p} / M_{J} \leq 2$ and $M_{p} / M_{J}<1 / 2$, respectively. The parameters used are $a=9.3 \mathrm{~km} \mathrm{~s}^{-1}$, appropriate to $T=10^{4} \mathrm{~K}, \mu=1$, and $R=R_{\mathrm{ph}}$.

by a factor of $10-10^{4}$, far less than for planets with cold upper atmospheres more distant from their parent star. This increased density leads to the possibility that hot Jupiter upper atmospheres may be collisional to large distances from the planet, i.e. that the exobase, if it exists at all, is at radii $r \gg R_{\mathrm{ph}}$ (Tian et al. 2005; Murray-Clay et al. 2009).

Next, note that for the same fiducial molecular weight and temperature, the strength of the tide, $\epsilon$, is in the range $0.1-1$ for a substantial number of planets. For large $\epsilon$, the typical rotational speed of a synchronized planet is comparable to the 


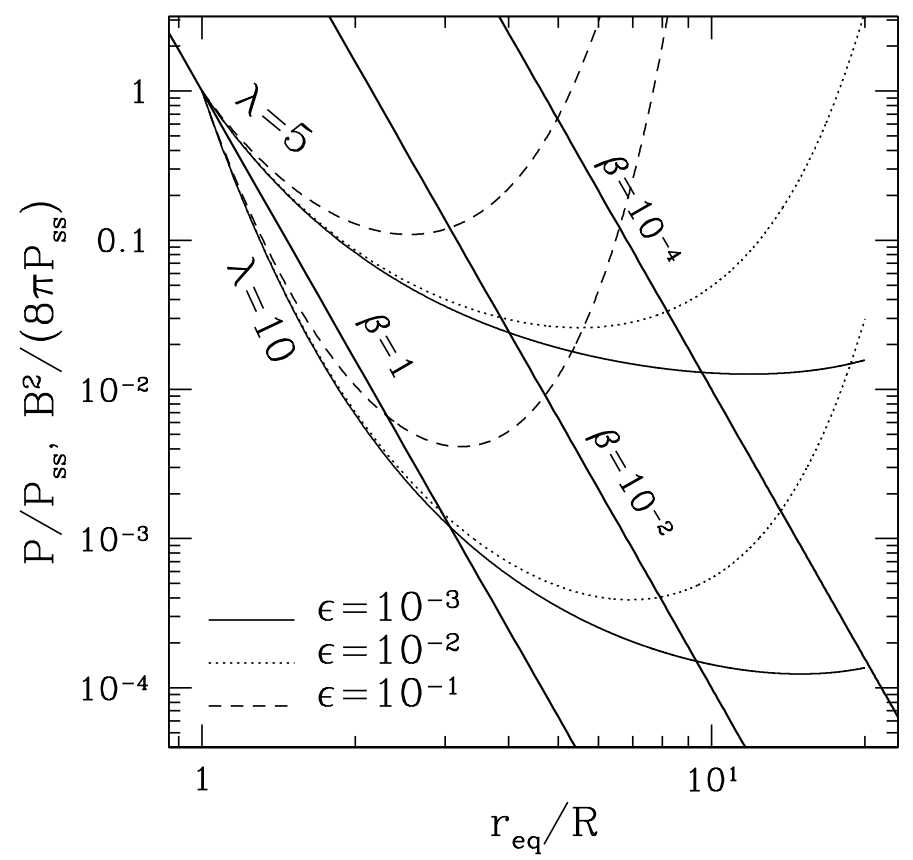

Fig. 2.3. - Gas and magnetic pressure, normalized to the base gas pressure $P_{s s}=$ $P(R, \pi / 2,0)$, as a function of equatorial radius $r_{\text {eq }}$ for the isothermal model. The tidal potential is evaluated along the star-planet line $\cos ^{2} \phi=1$ at the equator $\theta=\pi / 2$. The two groups of lines starting from $r=R$ and $P=P_{s s}$ are $P\left(r_{\mathrm{eq}}, \pi / 2,0\right) / P_{s s}$ evaluated for $\lambda=5,10$. For each group, the line style gives the value of $\epsilon$. The three lines sloping down to the right are $B^{2}\left(r_{\text {eq }}, \pi / 2,0\right) /\left(8 \pi P_{s s}\right)=\left(R / r_{\text {eq }}\right)^{6} / \beta$ for the three different values of equatorial $\beta=10^{-4}, 10^{-2}, 1$. The cusp radius in Figure 2.4 is given by the intersection of the gas and magnetic pressure curves. The gas pressure decreases outward faster for larger $\lambda$. Beyond the Roche radius, gravity effectively points outward and the gas pressure begins to increase outward. For larger $\epsilon$, the Roche radius moves inward.

sound speed, or equivalently, the free fall speed in the tidal potential is comparable to the sound speed. Since $\epsilon$ is evaluated at the base, the tidal force will dominate even more at larger distances from the planet.

Figure 2.3 shows the run of gas and magnetic pressure along the equator $(\theta=\pi / 2)$ as a function of radius along the star-planet line $(\phi=0)$. Eq.2.19 was used for the gas pressure, and Eq. 2.8 for the magnetic pressure. The magnetic pressure is 
parametrized by the plasma $\beta$ at the substellar point at the base:

$$
\beta \equiv \frac{8 \pi P_{s s}}{\left(B_{0} / 2\right)^{2}}=0.14\left(\frac{P_{s s}}{0.1 \mu \mathrm{bar}}\right)\left(\frac{B_{\mathrm{J}, \mathrm{eq}}}{B_{0} / 2}\right)^{2}
$$

The different lines in Figure 2.3 show gas pressure and magnetic pressure for different $\lambda, \epsilon$, and $\beta$ as a function of radius.

For small $\epsilon$, the density decreases outward, and eventually becomes a constant. When the tidal force is included the density increases outward for radii outside the magnetic Roche radius (Eq. 2.14), since the sign of gravity points outward there. This is a dramatic effect for close-in planets, whose Roche radii are at only a few planetary radii, and may lead to hydrogen densities orders of magnitude larger than the $\epsilon=0$ case. The tidal gravity plays a role similar to the centrifugal force in models of the closed field line regions in the solar wind (Mestel \& Spruit 1987) and in Jupiter's magnetosphere outside the corotation radius. To specify the current and field distributions required for this support involves a solution of the trans-field equation, which is beyond the scope of this work. However, such support should be possible when magnetic pressure dominates gas pressure.

Next, we follow Mestel (1968) and Mestel \& Spruit (1987) to estimate the size of the dead and wind zones. Pneuman \& Kopp (1971) showed that the dead zone ends at the equator in a cusp, i.e. the field in the dead zone approaches zero toward the cusp. Also, for $v \gtrsim a$, the wind zone pressure can be neglected compared to the dead zone pressure, leading to the condition (also see our discussion leading up to Eq. 2.83 in Appendix A)

$$
P_{\text {dead }} \simeq \frac{B_{\text {wind }}^{2}}{8 \pi}
$$




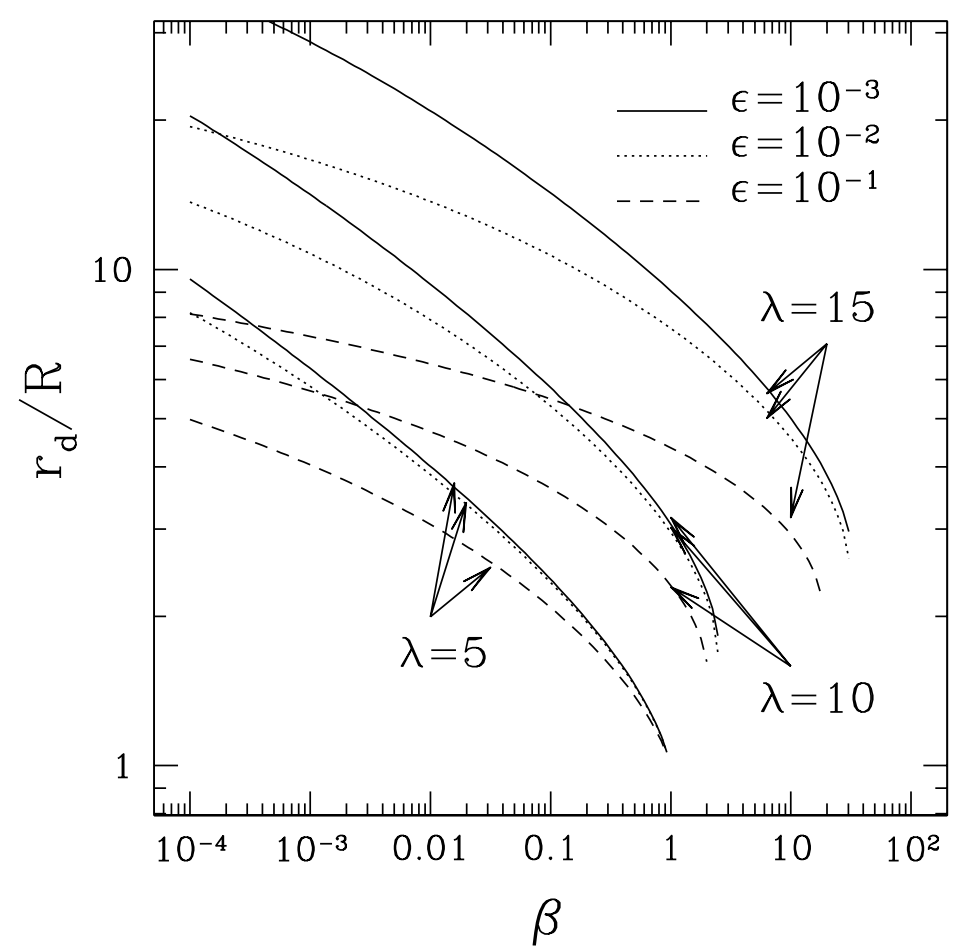

Fig. 2.4.- Cusp radius as a function of equatorial plasma $\beta$ at the substellar base of the atmosphere for different values of $\lambda=G M_{p} /\left(R a^{2}\right)$ and $\epsilon=(\Omega R / a)^{2}$. Here $\lambda$ is roughly the ratio of escape speed to isothermal sound speed, and $\epsilon$ is the roughly the ratio of rotation speed to isothermal sound speed. Larger $\lambda(\epsilon)$ implies the density decreases outward faster (slower). The cusp radius moves outward (inward) for larger $\lambda(\epsilon)$.

To determine the cusp radius, which we call $r_{d}$ for dead zone, we use Eq. 2.19 for the left hand side and Eq. 2.8 for the right hand side. The resulting equation is

$$
\beta \exp \left[-\lambda\left(1-\frac{R}{r}\right)+\frac{3}{2} \epsilon\left(\frac{r^{2}}{R^{2}} \cos ^{2} \phi-1\right)\right]=\left(\frac{R}{r}\right)^{-6}
$$

Eq.2.26 shows that the cusp radius will depend on $\phi$ in general due to the tidal potential. When tides can be ignored $(\epsilon \ll 1)$, a reasonable approximation is $r_{d} / R \simeq$ $\left(e^{\lambda} / \beta\right)^{1 / 6}$.

Figure 2.4 shows cusp radii as a function of $\beta$ for different tidal strength $(\epsilon)$ and binding parameter $(\lambda)$. Consider the fiducial case with $\epsilon=10^{-3}, \lambda=10$ and 
$\beta=10^{-2}$. Initially the magnetic pressure is much larger than the gas pressure. The more rapid decrease of magnetic pressure implies equality at the cusp radius, $r_{d} / R \simeq 10$. Increasing $\epsilon$, the gas pressure is larger and the cusp radius moves inward. The cusp radius moves outward with increasing magnetic field, i.e. decreasing $\beta$.

Under what conditions does a magnetosphere not form? Inspection of the $\beta=$ 1 and $\lambda=5$ curves in Figure 2.3 shows that the magnetic and gas pressures are initially equal at the base, but the gas density decreases more slowly and so the magnetic field never dominates. Ignoring tides, there is an analytic criterion for the critical $\beta_{\text {crit }}=\beta_{\text {crit }}(\lambda)$ for the formation of a dead zone. This criterion is found by requiring simultaneously $P=B^{2} / 8 \pi$ and $d P / d r=d / d r\left(B^{2} / 8 \pi\right)$, and yields $\beta \leq$ $\beta_{\text {crit }}=(6 / \lambda)^{6} \exp (\lambda-6)$. For $\lambda=5,10,15$, the values are $\beta_{\text {crit }}=1.1,2.5$ and 33, agreeing with the cutoffs in Figure 2.4. Hence for large $\lambda$, the gas at the base need not be magnetically dominated in order for the gas well above to base to become so.

In summary, we have found cusp, or dead zone, radii in the range of a few to tens of radii $R$ for the expected range of $\lambda, \beta$ and $\epsilon$. Since transit observations to date probe the high density gas within a few planetary radii, these observations may be probing static gas trapped within the magnetosphere, as opposed to outflowing gas.

Nevertheless, as we will argue in section 2.7, a wind zone should exist, and we investigate its structure in the next section.

\subsection{The Wind Zone}

In this section we show how the (slow magneto-) sonic point is affected by the magnetic geometry. Readers unfamiliar with the MHD wind equations can consult Appendix 2.15 for a brief summary of the equations. We simplify the problem by assuming that the sonic point is close enough to the planet for magnetic stresses to dominate 
over hydrodynamic stresses - the rigid field line approximation. In this situation the fluid nearly corotates with the planet, and can be accelerated like "beads on a wire" by the magnetic field. This "magneto-centrifugal" effect from stellar wind theory (Mestel 1968) becomes important when the rotation velocity approaches the sound speed at the sonic point (Mestel \& Spruit 1987). For a synchronized hot Jupiter, tidal forces are of comparable size as centrifugal forces, and the condition that the rotation velocity is comparable to the sound velocity at the sonic point is equivalent to the Roche-lobe radius being near the sonic point. The rigid field line assumption will typically break down near the Alfvén point, which we estimate lies well outside the dead zone radius for most latitudes.

Plugging parallel velocity, $\boldsymbol{v}=v \boldsymbol{b}$, and the no monopoles condition, Eq. 2.76, into Eq. 2.70, the continuity equation assumes the simple form

$$
\boldsymbol{B} \cdot \boldsymbol{\nabla}\left(\frac{\rho v}{B}\right)=0
$$

so that $\rho v / B$ is constant on field lines, and has the interpretation of the mass loss rate per unit of magnetic flux along a flux tube of area $\propto B^{-1}$. Similarly, the projection of Eq. 2.71 along the field can be rewritten as

$$
v \frac{d v}{d s}=-a^{2} \frac{d \ln \rho}{d s}-\frac{d U}{d s}
$$

which has the Bernoulli integral along field lines (see Eq. 2.77 and 2.78). Again, the $(\boldsymbol{J} \times \boldsymbol{B})$ force cancels out since $\boldsymbol{v} \| \boldsymbol{B}$. Combining Eq. 2.27 and 2.28 gives the momentum equation parallel to field lines:

$$
\left(v-\frac{a^{2}}{v}\right) \frac{d v}{d s}=-a^{2} \frac{d \ln B}{d s}-\frac{d U}{d s}
$$


At the critical point, $v=a$, and hence to avoid a divergent acceleration, the right hand side must go to zero, giving

$$
-a^{2} \frac{d \ln B}{d s}=\frac{d U}{d s}
$$

at the sonic point. The term on the left hand side represents the pressure gradient due to the geometry set by the magnetic field. The term on the right hand side is the net acceleration along the field line.

Since the sonic point is sufficiently close to the surface that the field geometry is not much perturbed by external currents, we use dipole geometry to evaluate the sonic point position. The dipole field in Eq. 2.8 gives the intermediate result

$$
-\frac{d \ln B}{d s}=\frac{3 \cos \theta}{N r}\left(1+\frac{\sin ^{2} \theta}{8 N}\right)
$$

The two terms on the right hand side of Eq. 2.31 represent the field line divergence due to the $r^{-3}$ factor from dipole field geometry, and the $\theta$-dependent factor $N$. The term due to differentiating $N$ is negligible for large loops, but becomes important for small loops near the equator. Plugging Eq. 2.11 and 2.31 into Eq. 2.30, and eliminating $\theta$ using the field line geometry in Eq. 2.12, we find the following equation to determine the sonic point $r=r_{s}$ :

$$
\frac{G M_{p}}{r^{2}}+\Omega^{2} r=\frac{3 a^{2}}{r}
$$

where $N=\sqrt{1-3 r / 4 r_{\text {eq }}}$. This equation, solely in terms of $r$, can be solved as a function of the parameters $\lambda, \epsilon$ and $r_{\mathrm{eq}} / R=1 / \sin ^{2} \theta_{b}$.

First we examine the limit in which tidal forces can be neglected. This simple 
case highlights the importance of the magnetic field geometry, and would apply for slow rotating planets distant from the star. Setting $\Omega=0$ in Eq. 2.32, the simpler equation

$$
\frac{G M_{p}}{3 a^{2} r}=1+\frac{r}{8 r_{\mathrm{eq}} \sqrt{1-3 r / 4 r_{\mathrm{eq}}}}
$$

results. For large field lines $r_{\text {eq }} \gg R$, field line curvature is negligible and the sonic point sits at

$$
r_{s 0} \simeq \frac{G M_{p}}{3 a^{2}}=\left(\frac{\lambda}{3}\right) R
$$

which differs from the spherical wind result by the 3 , instead of 2 , in the denominator. Including finite $\sin ^{2} \theta_{b}$, the sonic point moves inward somewhat.

Next, we include tidal forces, but ignore the field line curvature terms on the right hand side scaling as $r_{\mathrm{eq}}^{-1}$, a good approximation for field lines near the pole. This approximation eliminates the possibility that the tidal force can point outward. In this case, the sonic point equation becomes

$$
\frac{G M_{p}}{r^{2}}+\Omega^{2} r=\frac{3 a^{2}}{r} .
$$

The key point is that now the effective gravity on the left hand side has a minimum. If the pressure term on the right hand side is smaller than this minimum, a transonic solution is not possible. The solution of the cubic Eq. 2.35 lying near the planet 
disappears for sufficiently strong tidal forces

$$
\begin{aligned}
\Omega & \geq \Omega_{\text {crit }}=2 \frac{a^{3}}{G M_{p}}=\frac{2}{3} \frac{a}{r_{s 0}} \\
& =\left(\frac{2 \pi}{3.4 \text { days }}\right)\left(\frac{0.7 M_{J}}{M_{p}}\right)\left(\frac{a}{10 \mathrm{~km} \mathrm{~s}^{-1}}\right)^{3} .
\end{aligned}
$$

Eq.2.36 can also be written in dimensionless form as

$$
\epsilon_{\text {crit }}=\frac{4}{\lambda^{2}}
$$

When Eq. 2.36 is satisfied, for planets sufficiently close to the star, there is no sonic point solution, and the wind is shut off near the poles. The density distribution on these field lines will be hydrostatic. Hence, for sufficiently strong tides such that the rotation velocity $\Omega_{\text {crit }} r_{s 0}$ at the sonic point is supersonic, a second dead zone is created in the polar regions where tides point inward. Whereas the first dead zone in the equatorial region is due to magnetic pressure dominating gas pressure, the second dead zone near the poles arises due to the large potential barrier.

Figure 2.5 shows $\epsilon$ versus $\lambda$ for the observed transiting planets. Except for a handful of planets with the smallest values of both $\epsilon$ and $\lambda$, most of the planets are in the strong tide limit with $\epsilon>\epsilon_{\text {crit }}$. The planets in the upper right hand corner will have the polar wind partially shut off, while the planets in the lower left hand corner will be able to drive a polar wind.

Next, we retain the $r_{\text {eq }}^{-1}$ terms due to the tidal force in Eq. 2.32. Taking the limit $\Omega \rightarrow \infty$ in Eq. 2.32, the sonic point in the strong tide limit is

$$
r_{s} \rightarrow \frac{2 r_{\mathrm{eq}}}{3 f}
$$




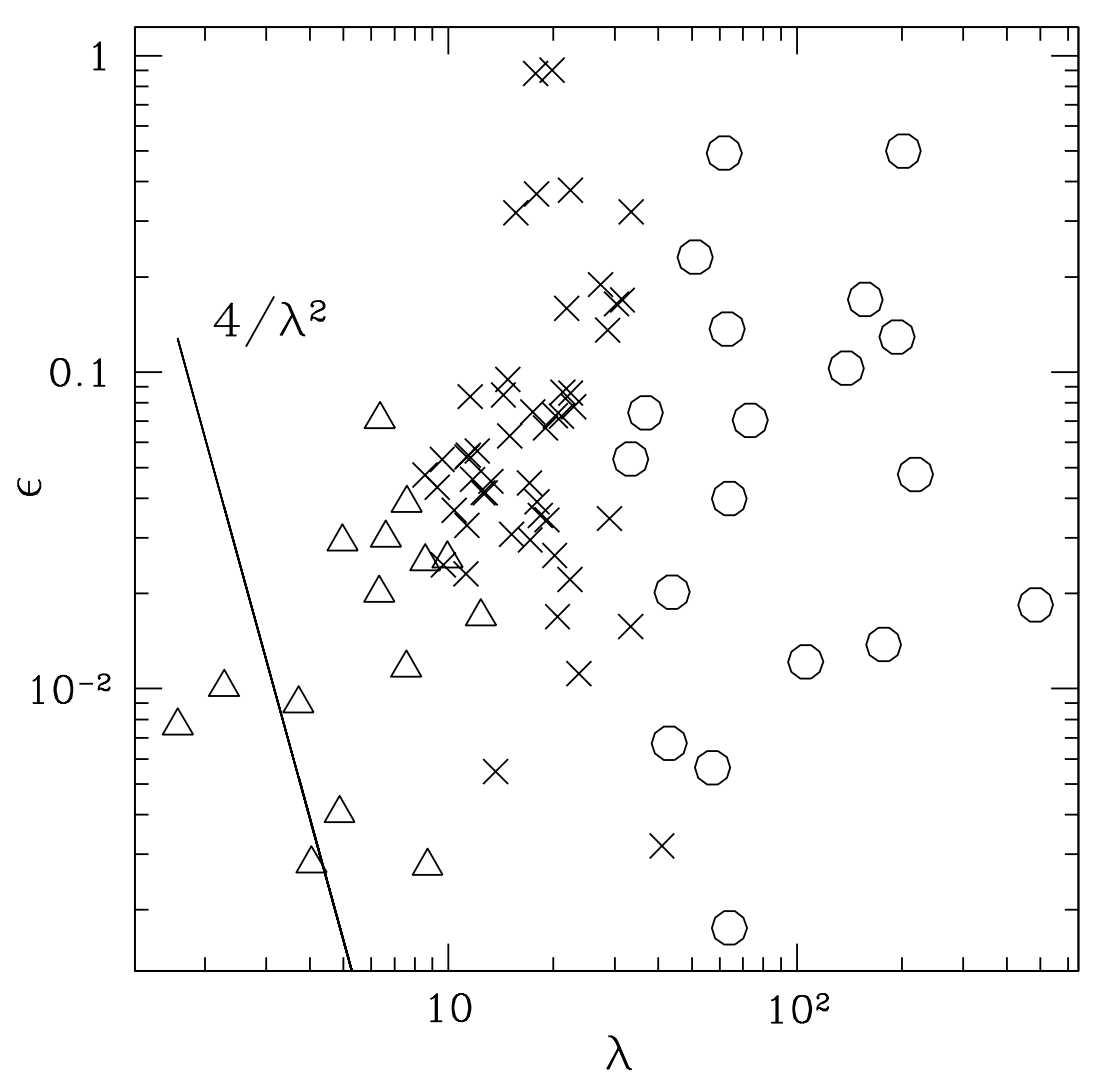

Fig. 2.5. - Values of $\epsilon$ versus $\lambda$ for the transiting planets. The points are the data, with symbol type as in Figures 2.1 and 2.2. The line is the critical tidal strength $\epsilon=4 / \lambda^{2}$ above which the wind is suppressed in the polar region. We have used $a=9.3 \mathrm{~km} \mathrm{~s}^{-1}$ to make the plot.

In this limit, the sonic point occurs at a fixed fraction of the loop equatorial radius, dependent only on $\cos ^{2} \phi$, and agrees with the magnetic Roche lobe radius found in Eq. 2.14, where the net force first points outward.

Figure 2.6 shows an example numerical solution of Eq. 2.32 for the sonic point radius as a function of footpoint angle $\theta_{b}$. Dipole geometry was used to produce this plot. In the weak tide limit $(\epsilon \rightarrow 0)$, the solutions asymptote to Eq. 2.34 for large loop size, and decrease slightly before terminating at the dead zone, $\theta=\theta_{d}$. For small $\epsilon \lesssim 4 / \lambda^{2}$, the sonic point moves out in the polar regions, and inward closer to the equator; the dividing line between these two behaviors depends on if the net 


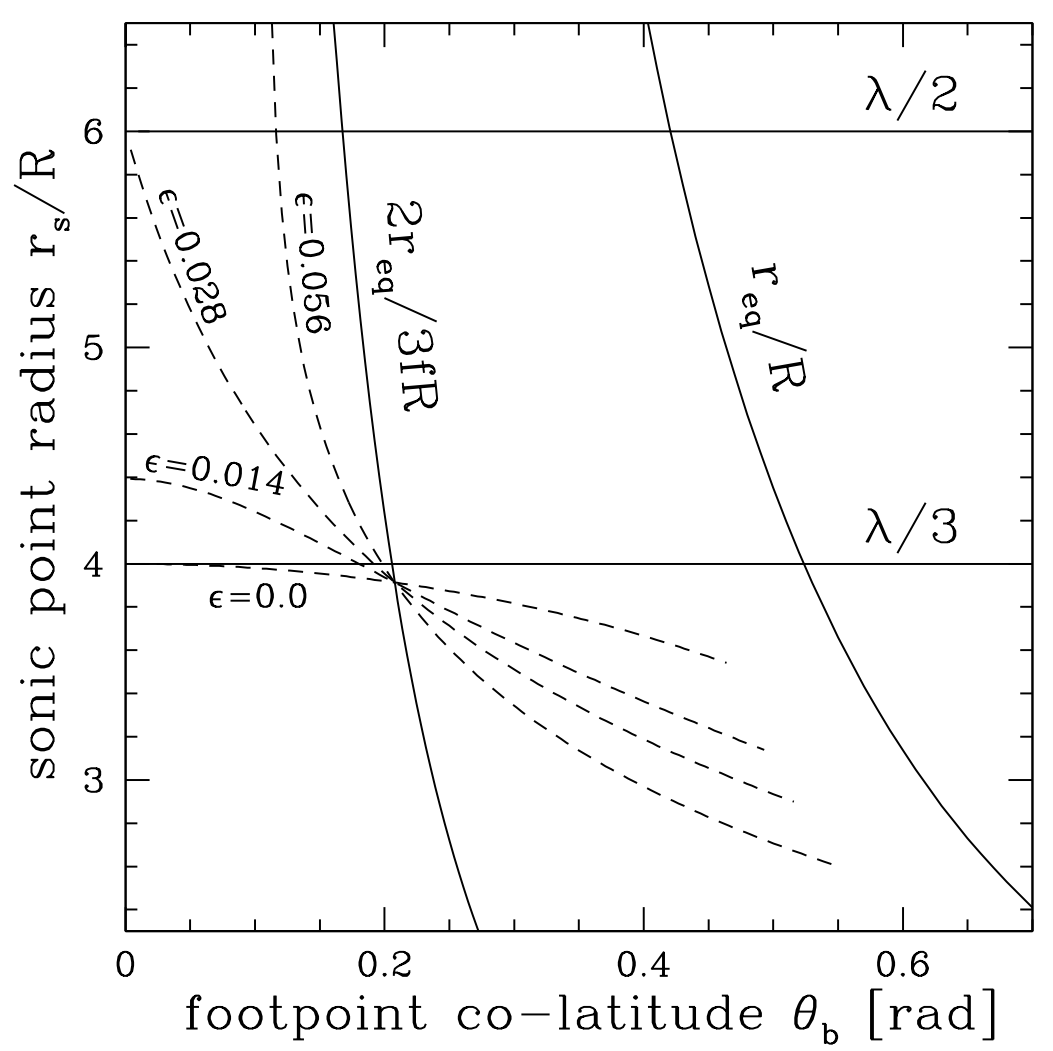

Fig. 2.6. - Sonic point radius as a function of footpoint co-latitude for $\lambda=12, \beta=1$ and a range of $\epsilon$ (dashed lines). The three solid lines label the $\epsilon=0$ sonic point, $r_{s 0} / R=\lambda / 3$, the $\epsilon=4 / \lambda^{2}=0.16$ sonic point, $r_{s} / R=\lambda / 2$, and the $\epsilon=\infty$ sonic point, $r_{s}=2 r_{\text {eq }} / 3 f$. Each dashed line terminates at large $\theta$ at the dead zone, $\theta=\theta_{d}$. The dead zone shrinks $\left(\theta_{d} \rightarrow \pi / 2\right)$ as $\epsilon$ increases. The value of $\beta$ is needed only for the size of the dead zone. The longitude $\cos ^{2} \phi=1$, along the star-planet line, has been assumed here.

force is outward or inward near the looptop. Next, for the critical value $\epsilon=4 / \lambda^{2}$, the sonic point is at roughly $r_{s} \simeq(\lambda / 2) R$ near the pole. For larger values $\epsilon \gtrsim 4 / \lambda^{2}$, the sonic point near the pole jumps out to a radius much further from the planet, near $r_{s} \sim 2 r_{\text {eq }} / 3 f$, where the net gravity changes sign. For small values of $\theta_{b}$, this solution may be at tens to hundreds of planetary radii, and is of no physical interest. Physically, when the sonic point moves outside the region of interest the field line is effectively hydrostatic. 


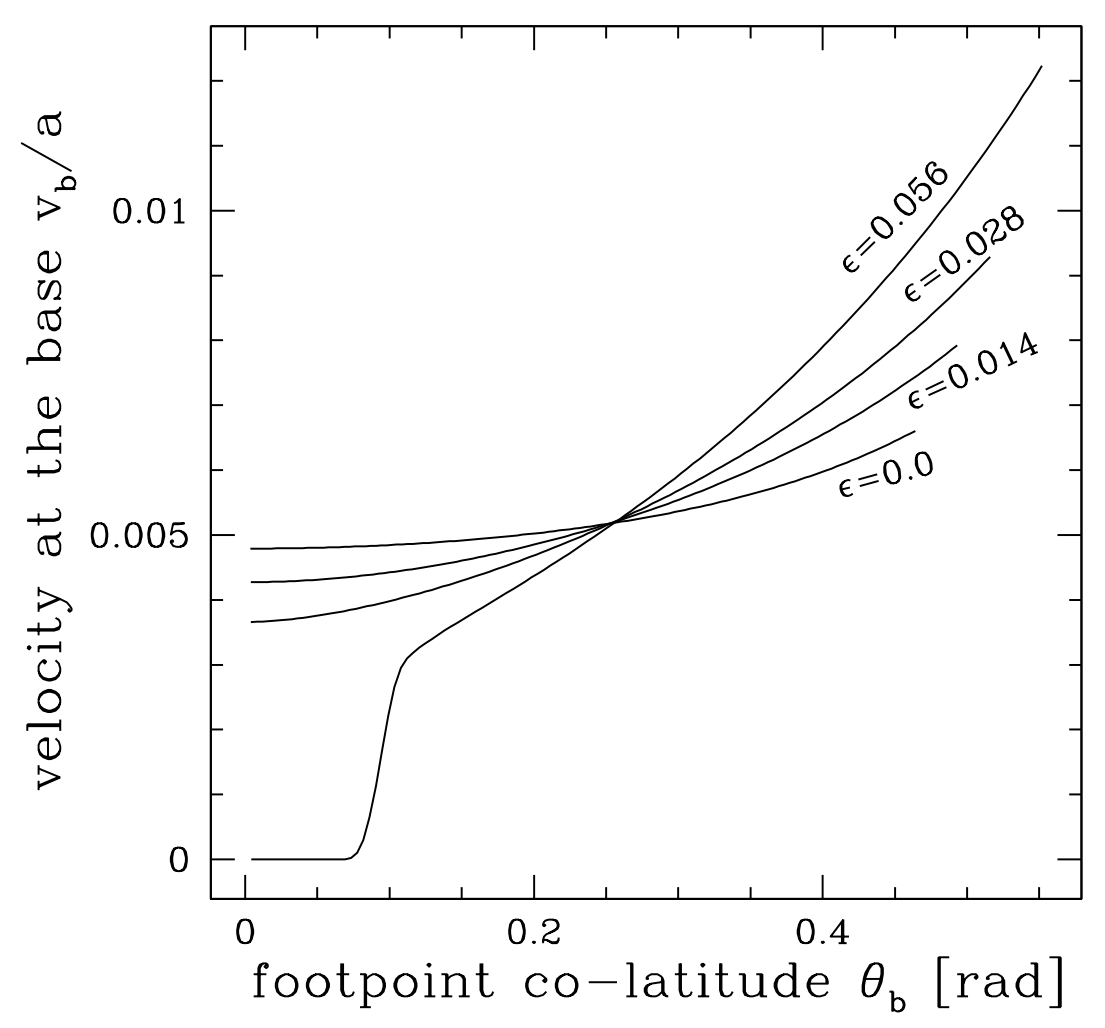

Fig. 2.7.- Velocity at the base, in units of $a$, for the same parameters as Figure 2.6.

Figure 2.7 shows the velocity at the base, $v_{b}$, for the same parameters as in Figure 2.6. This velocity was found by using the Bernoulli constant evaluated at the sonic point and the base. For weak tides $(\epsilon=0)$, the velocity at the base is nearly constant over the wind zone. As $\epsilon$ increases, the base velocity decreases near the pole, where the sonic point has moved outward, and increases closer to the equator, where the sonic point has moved inward.

At sufficiently large radii, pressure gradients rapidly become negligible and the fluid should move on a nearly ballistic trajectory. Using the Bernoulli integral defined 
in Eq. 2.78, with the sonic point as reference location, the velocity parallel to the magnetic field is

$$
v^{2}=a^{2}+2 a^{2} \ln \left(\frac{B_{s}}{B} \frac{v}{a}\right)+2\left(U_{s}-U\right)
$$

where the subscript "s" refers to the sonic point. The transit signal depends only on the gas velocity within the stellar disk at $r \leq R_{\star}$, where $R_{\star}$ is the stellar radius. The tidal potential term $-2 U$ dominates at large radius. For the purposes of a simple estimate, this asymptotic expression, evaluated at the stellar radius, gives

$$
\begin{aligned}
v_{\text {asymp }} & \simeq \Omega R_{\star}\left(f \sin ^{2} \theta-1\right)^{1 / 2} \\
& =24 \mathrm{~km} \mathrm{~s}^{-1}\left(\frac{3.6 \text { day }}{P_{\text {orb }}}\right)\left(\frac{R_{\star}}{R_{\odot}}\right)\left(\frac{f \sin ^{2} \theta-1}{3}\right)^{1 / 2}
\end{aligned}
$$

We caution the reader that the (logarithmic) enthalpy term in Eq. 2.39 is not negligible, and can increase the velocity at $r=R_{\star}$ by a factor of order 2 (see Figure 2.10). Though supersonic, the velocity in Eq. 2.40 is much smaller than the $\sim \pm 100 \mathrm{~km} \mathrm{~s}^{-1}$ at which absorption is observed in the Lyman $\alpha$ spectrum of HD 209458b (?), and hence velocity gradients cannot be the origin of the observed transit depth.

In the following sections, we will implement the general theory that describes the structure of the dead zone $(\S 2.6)$ and of the wind zone $(\S 2.7)$ to construct global models of hot Jupiter magnetospheres for comparison with transit observations of HD 209458b. A simplified 1D thermal model motivates our choice of the pressure and sound speed at the base of the global models, which are key parameters for determining the magnetospheric structure. 


\section{8 $\mathrm{H}$ and $\mathrm{H}^{+}$Layers: A Simplified 1D Thermal Model}

The thickness of the warm $\mathrm{H}$ layer with temperature $T \simeq 5,000-10,000 \mathrm{~K}$ is a crucial parameter in determining the transit depth, as emphasized in the phenomenological model of Koskinen et al. (2010) (see García Muñoz (2007) for a discussion of the role of base pressure for an atmosphere undergoing energy-limited escape). The large temperature and small mean molecular weight increase the scale height to $\sim 0.1 R_{\text {ph }}$. If the warm layer extends over $\gtrsim 10$ scale heights, the transit radius can be significantly increased over the photospheric radius of the optical continuum. In this section, we construct a simple 1D model in photoionization and thermal equilibrium to determine the depth of the warm H layer.

At sufficiently low density and temperature, the rates of collisional ionization and 3-body recombination are slow compared to photoionization and radiative recombination, respectively, and the ionization state is set by a balance of the latter two processes. We consider a pure hydrogen gas for simplicity. Let $n_{e}, n_{p}$ and $n_{H}$ be the density of electrons, protons and hydrogen atoms. Charge neutrality implies $n_{e}=n_{p}$. The ionization fraction in the "on the spot" approximation is found by solving the algebraic equation (Osterbrock \& Ferland 2006)

$$
n_{H} J\left(N_{H}\right)=\alpha_{B}(T) n_{e} n_{p}=\alpha_{B}(T) n_{p}^{2} .
$$

At the $50 \%$ ionization point,

$$
n_{H}=n_{p} \equiv n_{\mathrm{eq}}=\frac{J}{\alpha_{B}} .
$$


Here $\alpha_{B}(T) \simeq 2.6 \times 10^{-13} \mathrm{~cm}^{3} \mathrm{~s}^{-1}\left(10^{4} \mathrm{~K} / T\right)^{0.8}$ is the case $\mathrm{B}$ radiative recombination rate (Osterbrock \& Ferland 2006),

$$
J\left(N_{H}\right)=\int_{\nu_{0}}^{\infty} d \nu \varphi_{\nu} e^{-N_{H} \sigma_{\mathrm{pi}}(\nu)} \sigma_{\mathrm{pi}}(\nu)
$$

is the photoionization rate per $\mathrm{H}$ atom, $\varphi_{\nu}$ is the photon flux per unit frequency interval, $N_{H}$ is the atomic hydrogen column from the point in question to the star, $\sigma_{\mathrm{pi}}(\nu)=\sigma_{\mathrm{pi}}\left(\nu_{0} / \nu\right)^{3}$ is the $\mathrm{H}$ atom bound-free cross section, the threshold cross section is $\sigma_{\mathrm{pi}}=6.3 \times 10^{-18} \mathrm{~cm}^{2}$, and the threshold frequency is $\nu_{0}=13.6 \mathrm{eV} / \mathrm{h}$. The exponential factor in Eq. 2.43 takes into account attenuation of the stellar radiation. Eq.2.43 may be computed as a function of $N_{H}$, as described in Osterbrock \& Ferland (2006).

For the thermal balance, the dominant processes are photoelectric heating from the ionization of hydrogen atoms (Yelle 2004), and cooling by collisionally excited Lyman $\alpha$ emission from electron impacts (Murray-Clay et al. 2009). Assuming 100\% efficiency of turning photoelectron energy into heat, the heating rate per reaction is

$$
Q\left(N_{H}\right)=\int_{\nu_{0}}^{\infty} d \nu \varphi_{\nu} e^{-N_{H} \sigma_{\mathrm{pi}}(\nu)} \sigma_{\mathrm{pi}}(\nu) h\left(\nu-\nu_{0}\right)
$$

Balancing photoelectric heating and cooling by collisionally excited Lyman $\alpha$ emission implies

$$
n_{H} Q\left(N_{H}\right)=\Lambda(T) n_{e} n_{H}
$$

Note that $n_{H}$ cancels out of Eq. 2.45. The line cooling coefficient for Lyman $\alpha$ is $\Lambda(T)=2.9 \times 10^{-19} \mathrm{erg} \mathrm{cm}^{3} \mathrm{~s}^{-1} \sqrt{10^{4} \mathrm{~K} / T} \exp (-118,400 \mathrm{~K} / T)$ (Dalgarno \& Mc- 
Cray 1972). Increasing distance from the star and decreased heating efficiency act to decrease $Q$.

Eq. 2.41 and 2.45 are two algebraic equations which can be solved for $n_{H}$ and $n_{p}$ in terms of $N_{H}$ and $P=\left(2 n_{p}+n_{H}\right) k_{b} T$. In practice, we assume a trial $T$, compute $n_{H}$ and $n_{p}$ from Eq. 2.41, and then compute the imbalance of heating and cooling in Eq. 2.45. The temperature is iterated until thermal balance is achieved. The equations for dependent variables $N_{H}$ and $P$ and independent variable $r$ are the definition of column

$$
\frac{d N_{H}}{d r}=-n_{H}
$$

and hydrostatic balance

$$
\frac{d P}{d r}=-\frac{G M_{p} m_{p}}{r^{2}}\left(n_{H}+n_{p}\right)
$$

where tides have been ignored for simplicity. The inner boundary condition is $r=R$ at the chosen base pressure. The column $N_{H}$ should go to zero at the outer boundary. We enforce this boundary condition at the finite, but large, radius $r=40 R$.

To compute the integrals in Eq. 2.43 and Eq. 2.44, we use the quiet solar Lyman continuum spectrum from Woods et al. (1998), which tabulates $\int d \nu \varphi_{\nu}$ in each frequency bin. The results are shown as the solid lines in Figure 2.8. Approximate power-law fits are also shown, along with curves representing pure exponential attenuation for comparison. A similar fit with shallower slope is shown in the bottom panel for the heating rate $Q\left(N_{H}\right)$. At small optical depth, $J_{0} \approx(6 \mathrm{hr})^{-1}$ and the mean photoelectron energy is $Q_{0} / J_{0} \simeq 2.7 \mathrm{eV}$. Given the form of the integrand in Eq. 2.43, one might have expected an exponential scaling of the form $J \propto \exp \left[-(\right.$ constant $\left.) \sigma_{\mathrm{pi}} \mathrm{N}_{\mathrm{H}}\right]$, 
implying negligible heating and ionization deep in the $\mathrm{H}$ layer. This is shown as the dot-dashed line in Figure 2.8, and cuts off much too sharply. The numerical result is better fit with a power-law, $J \propto N_{H}^{-1.5}$, leading to larger heating rate deep into the H layer.

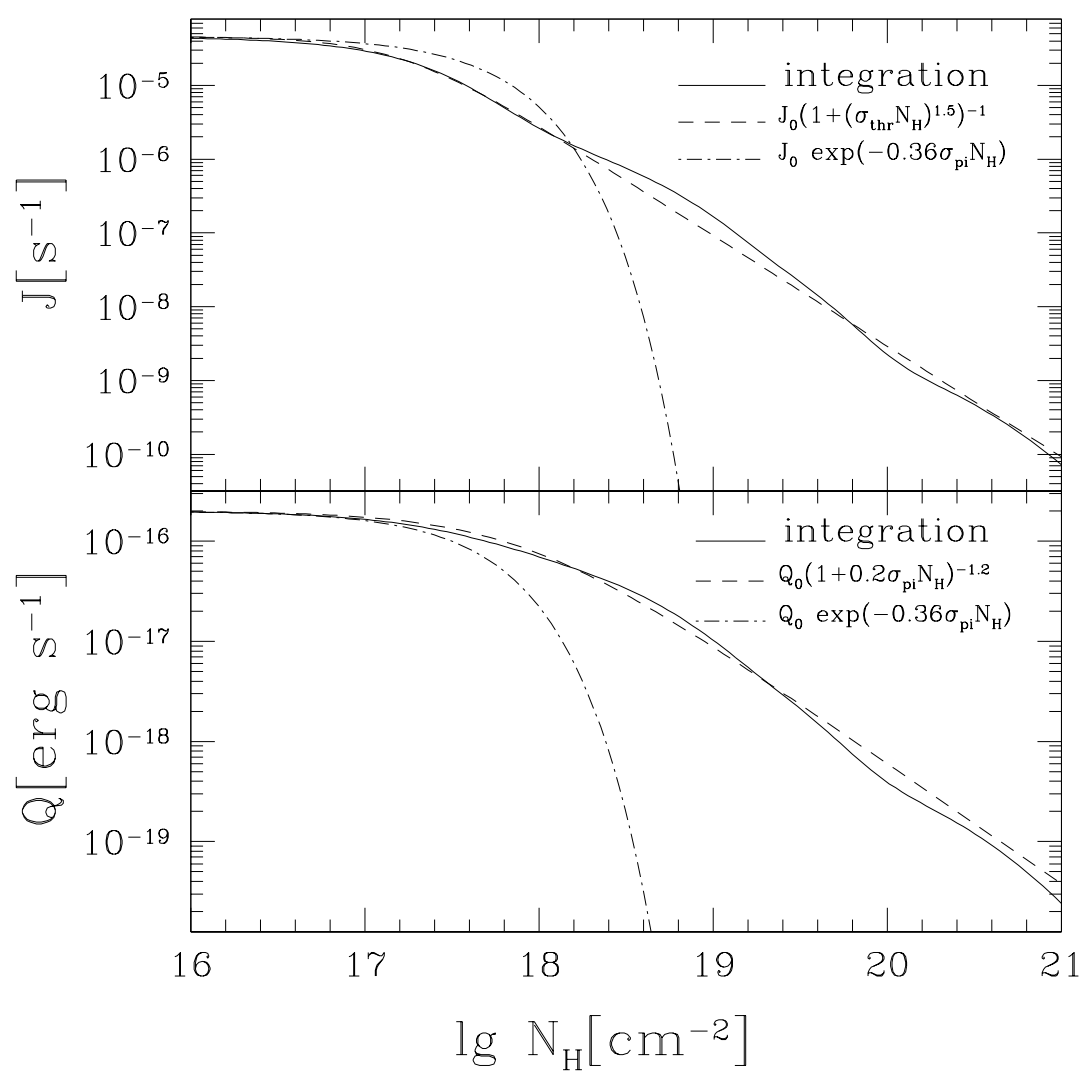

Fig. 2.8. - Photoionization rate (top) panel and heating rate (bottom) as a function of neutral hydrogen column, for a planet at $D=0.05$ AU around a solar-type star..

The weak scaling of $J$ with $N_{H}$ can be explained as the competition between $\exp \left[-N_{H} \sigma_{\mathrm{pi}}(\nu)\right]$, which increases to higher frequencies, and $\varphi_{\nu}$, which on average decreases to higher frequency. Ignoring lines in $\varphi_{\nu}$, the product of these two functions is a sharp peak, similar to the Gamow peak in thermonuclear reaction rates. For instance, if one approximates the Lyman continuum as a blackbody with temperature $T=8300 \mathrm{~K}$ (Noyes \& Kalkofen 1970), steepest descent evaluation of Eq. 2.43 gives 
the weak exponential scaling $J \propto \exp \left[-15.9\left(N_{H} \sigma_{\mathrm{pi}}\right)^{1 / 4}\right]$, which involves $N_{H}^{1 / 4}$ in the exponent rather than $N_{H}$. However, the blackbody fit is not adequate, as even this weaker scaling cuts off too fast. We find a better fit is to use a power-law form $\varphi_{\nu} \propto$ $\nu^{-\gamma}$, leading to $J \propto N_{H}^{-(\gamma+2) / 3}$, with no exponential scaling. Choosing $\gamma=2.5$ then recovers the observed scaling. While these analytic scalings are useful for intuition, the solar Lyman continuum contains many strong lines, and is not well approximated by a smooth continuum function when computing $J$.

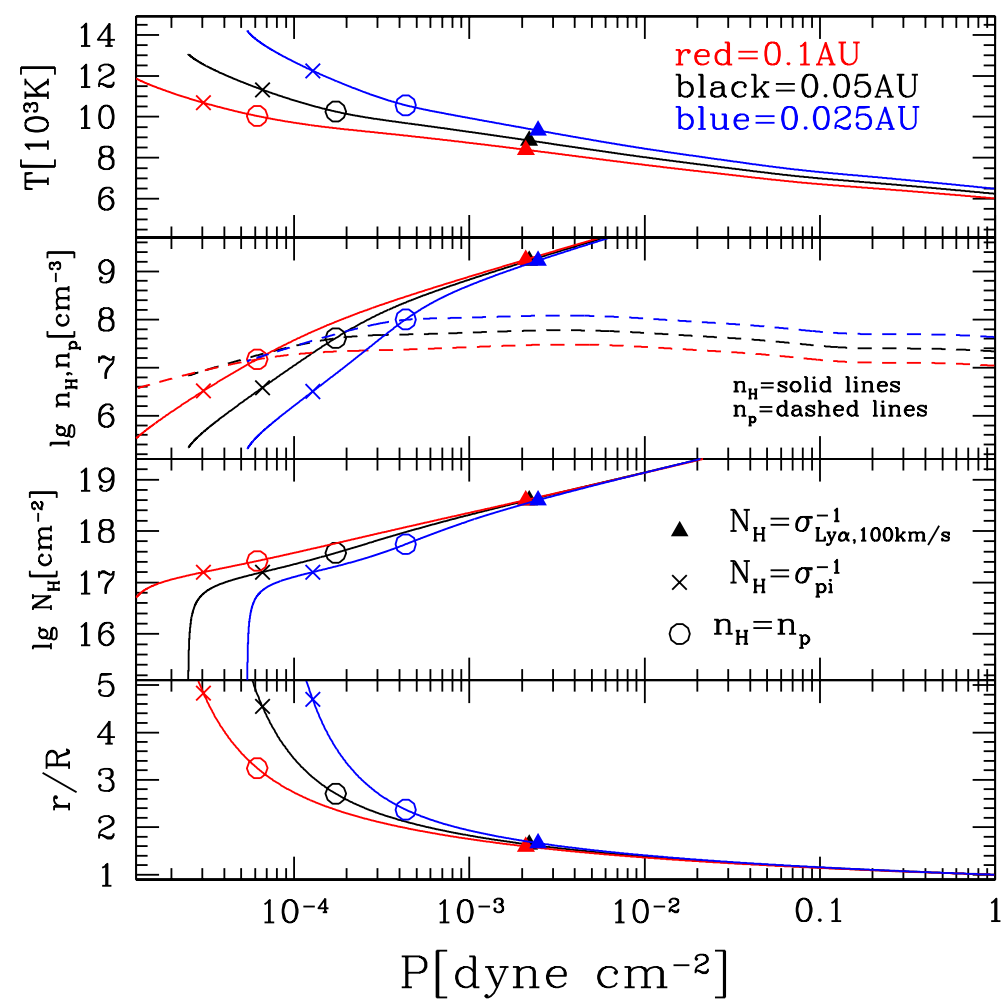

Fig. 2.9.- Temperature (top panel), number densities (second panel), neutral hydrogen column (third panel), and radius (bottom panel) versus pressure for a $M_{p}=0.7 M_{J}, R=1.3 R_{J}$ planet. The red, black and blue lines are for planets at $D=0.1,0.05,0.025 \mathrm{AU}$, respectively. The hollow circles show the position where Lyman continuum radiation (at threshold) from the star becomes optically thick. The crosses show the $50 \%$ ionization point. The filled triangles show the position in the atmosphere where stellar Lyman $\alpha$ photons at $\pm 100 \mathrm{~km} \mathrm{~s}^{-1}$ from line center become optically thick. 
Figure 2.9 shows a numerical integration of eqs. 2.46, 2.47, 2.41 and 2.45. Spherically symmetric irradiation is assumed, the base pressure is chosen to be $P=$ 1 dyne $\mathrm{cm}^{-2}$, and the $N_{H} \rightarrow 0$ condition is applied at the (arbitrary) radius $r=$ $40 R_{\mathrm{ph}}$. Parameters appropriate for HD 209458b have been chosen with $M_{p}=0.7 M_{J}$ and $R=1.3 R_{J}$. In each panel, the three different color curves are for different semimajor axis; the semi-major axis of $\mathrm{HD} 209458 \mathrm{~b}$ is $D \simeq 0.05 \mathrm{AU}$. In the top panel, note that $T$ increases slowly as the planet is moved closer to the star. A factor 16 increase in stellar flux translated into only a 10-20\% increase in the pressure range of interest, due to the exponential $T$ dependence in the cooling rate. Eq.2.45 can be solved analytically as

$$
T \simeq \frac{1.1 \times 10^{4} \mathrm{~K}}{1+0.089 \ln \left[\left(n_{e} / n_{\mathrm{eq}, 0}\right)\left(Q_{0} / Q\right)\right]}
$$

This simple estimate shows that the temperature is always near $10^{4} \mathrm{~K}$ near the base of the photoionized layer where $n_{H}=n_{p} \simeq n_{\mathrm{eq}}$. As $n_{e}$ decreases below $n_{\mathrm{eq}}$, the temperature rises logarithmically slowly. This temperature rise toward small density will increase the scale height and density at larger radii relative to an isothermal model with the same base conditions.

The second panel shows the rapid inward increase of $n_{H}$, with a change in slope at the $\mathrm{H}-\mathrm{H}^{+}$boundary. The slow decrease of $n_{p}$ into the atmosphere is due to the slow decrease of $J$ with $N_{H}$. If we had used the exponential scaling $J \propto \exp \left(-N_{H} \sigma_{\mathrm{pi}}\right)$ shown in Figure 2.8, $T$ and $n_{p}$ would have decreased inward much more rapidly. The hollow circles show the position of $n_{H}=n_{p}$ at each $D$. Planets further from the star remain neutral higher up in the atmosphere.

The third panel shows $N_{H}$, and x's show the position of $N_{H} \sigma_{\mathrm{pi}}=1$, where the Lyman continuum at threshold is optically thick. The optical depth unity point moves 
to lower pressure for planets more distant from the star, although the radius at this point is relatively constant.

The bottom panel shows radius in units of base radius. The warm $\mathrm{H}$ layer with temperature $T \simeq 10,000 \mathrm{~K}$ at pressures $P=(0.1-100)$ nbar contributes an amount $\simeq(1-2) \times R_{\mathrm{ph}}$ to the radius. Specifically, even the region below $N_{H} \sigma_{\mathrm{pi}}=1$ can be warm enough to contribute significantly to the radius. In our reference global model below, we use a base pressure $P_{\mathrm{ss}}=40$ nbar (see Model 1 of Table 1 ).

We now discuss how the simple 1D model differs from previous investigations. One crucial difference is that the dead zone should be hotter than the wind zone, for which adiabatic expansion is an important coolant. Yelle (2004) included heating arising from photoionization of hydrogen, but ignored the attenuation of the stellar Lyman continuum into the atmosphere, leading to an overestimate of the heating rate. This attenuation of EUV in the $\mathrm{H}$ layer will also lead to smaller heating by $\mathrm{H}_{2}$ photoionization much deeper in the atmosphere. Murray-Clay et al. (2009) included finite optical depth, but enforced an exponential cutoff which led to a steep temperature drop below the $N_{H} \sigma_{\mathrm{pi}}=1$ point. As shown in Figure 2.8, the exponential cutoff is too rapid, and the slower power-law cutoff found here gives additional heating deeper in the atmosphere.

Figure 2.9 shows that the $n_{H}=n_{p}$ and $N_{H} \sigma_{\mathrm{pi}}=1$ points occur near each other at $D=0.05 \mathrm{AU}$, hence attenuation due to finite optical depth cannot be ignored. The relative position of the $n_{H}=n_{p}$ and $N_{H} \sigma_{\mathrm{pi}}=1$ layers can be estimated as follows. The number density at which $n_{p}=n_{H}$ is

$$
\begin{aligned}
n_{\mathrm{eq}} & =\frac{J}{\alpha_{B}} \\
& \simeq 1.8 \times 10^{8} \mathrm{~cm}^{-3}\left(\frac{T}{10^{4} \mathrm{~K}}\right)^{0.8}\left(\frac{0.05 \mathrm{AU}}{D}\right)^{2}\left(\frac{J}{J_{0}}\right) .
\end{aligned}
$$


This can be converted into a pressure as

$$
\begin{aligned}
P_{\mathrm{eq}} & =3 k_{b} T n_{\mathrm{eq}} \\
& =8.0 \times 10^{-4} \text { dyne } \mathrm{cm}^{-2}\left(\frac{M_{p}}{M_{J}}\right)\left(\frac{10^{10} \mathrm{~cm}}{R}\right)\left(\frac{10}{\lambda}\right) \\
& \times\left(\frac{0.05 \mathrm{au}}{D}\right)^{2}\left(\frac{T}{10^{4} \mathrm{~K}}\right)^{0.8}\left(\frac{J}{J_{0}}\right)
\end{aligned}
$$

The $D^{-2}$ scaling implies that the atmosphere becomes neutral out to smaller pressures as the planet moves away from the star. To estimate where $N_{H} \sigma_{\mathrm{pi}}=1$, we assume $n_{p} \gg n_{H}, P \simeq 2 k_{b} T n_{p}$, and Eq. 2.41 gives $n_{H} \simeq n_{p}^{2} / n_{\mathrm{eq}, 0}$, where $n_{\mathrm{eq}, 0}=J_{0} / \alpha_{B}$. For $\mathrm{H}$ atom scale height $\simeq k_{b} T / m_{p} g$, the pressure at which $N_{H} \sigma_{\mathrm{pi}}=1$ is

$$
P_{\mathrm{N}_{\mathrm{H}} \sigma_{\mathrm{pi}}=1} \simeq 2 k_{b} T\left(\frac{n_{\mathrm{eq}, 0} m_{p} g}{k_{b} T \sigma_{\mathrm{pi}}}\right)^{1 / 2} \propto D^{-1}
$$

The scaling with $D$ in Eq. 2.51 implies that planets further from the star will become optically thick at lower pressure. Comparing the scalings in Eq. 2.50 and 2.51, one may expect $50 \%$ ionization to occur outside $N_{H} \sigma_{\mathrm{pi}}=1$ at sufficiently large $D$.

Lastly, we note that since the cooling rate $\propto n_{e} n_{H}$, we may expect Lyman $\alpha$ cooling to be inefficient at both high and low density, where other cooling mechanisms, such as heat conduction, may be more efficient.

\subsection{Global Models}

We now attempt to construct global models for the magnetosphere, in order to compute the planetary transmission spectrum and mass loss rates. We assume the fol- 
lowing magnetic field model (Mestel 1968; Okamoto 1974):

$$
\boldsymbol{B}(r, \theta)=\left\{\begin{array}{c}
B_{0}\left(\frac{R}{r}\right)^{3}\left(\boldsymbol{e}_{r} \cos \theta+\frac{1}{2} \boldsymbol{e}_{\theta} \sin \theta\right), \quad\left(r<r_{\mathrm{d}}\right) \\
B_{0}\left(\frac{R}{r_{\mathrm{d}}}\right)^{3}\left(\frac{r_{\mathrm{d}}}{r}\right)^{2} \cos \theta \boldsymbol{e}_{r}, \quad\left(r>r_{\mathrm{d}}\right) .
\end{array}\right.
$$

This global field model allows a position $(r, \theta)$ to be associated with a base co-latitude $\theta_{b}$ at $r=R$. The field is dipole for $r<r_{d}$, and radial outside $r_{d}$. This radial field is distinct from the split monopole due to the $\cos \theta$ factor. Eq.2.52 is approximately correct for the dead zone, and also for determining the sonic point position in the wind zone if the sonic point is close to the planet. However, as ballistic trajectories with speeds far less than escape speed are expected to be bent down toward the orbital plane, the radial field assumption will be unphysical for some latitudes.

Given parameters $\lambda, \epsilon, \beta$, and longitude $\phi$, we first solve Eq. 2.26 for $r_{d}$ using dipole geometry. For field lines inside the dead zone, $\sin \theta_{b}>\sin \theta_{d}=\sqrt{R / r_{d}}$, the velocity is zero and the density is given by the hydrostatic expression in Eq. 2.19, where $P_{\mathrm{ss}}$ is a model parameter. On field lines outside the dead zone, $\sin \theta_{b}<\sin \theta_{d}=\sqrt{R / r_{d}}$, we search for sonic points by looking for minima of the quantity $-\ln \left(B / B_{0}\right)-U / a^{2}$ on field lines, between the base radius $R$ and a chosen maximum radius $r_{\max }$. Given the position of the sonic point $\left(r_{s}, \theta_{s}\right)$, the Bernoulli equation $v^{2} / 2+a^{2} \ln (B / v)+U=$ constant can be used to solve for the velocity at the base, $v_{b}\left(\theta_{b}, \phi\right)$. Given $v_{b}$ and the base density (Eq. 2.18), the Bernoulli equation may again to used to find the run of $v$ and $\rho$ on the field line.

Detailed results will be presented for the 9 models listed in Table 2.1. The planetary mass and radius, and the stellar mass and radius are characteristic of HD 209458b. We vary parameters not directly measured, such as $P_{\mathrm{ss}}, a, B_{0}$, as well as the orbital radius $D$. 


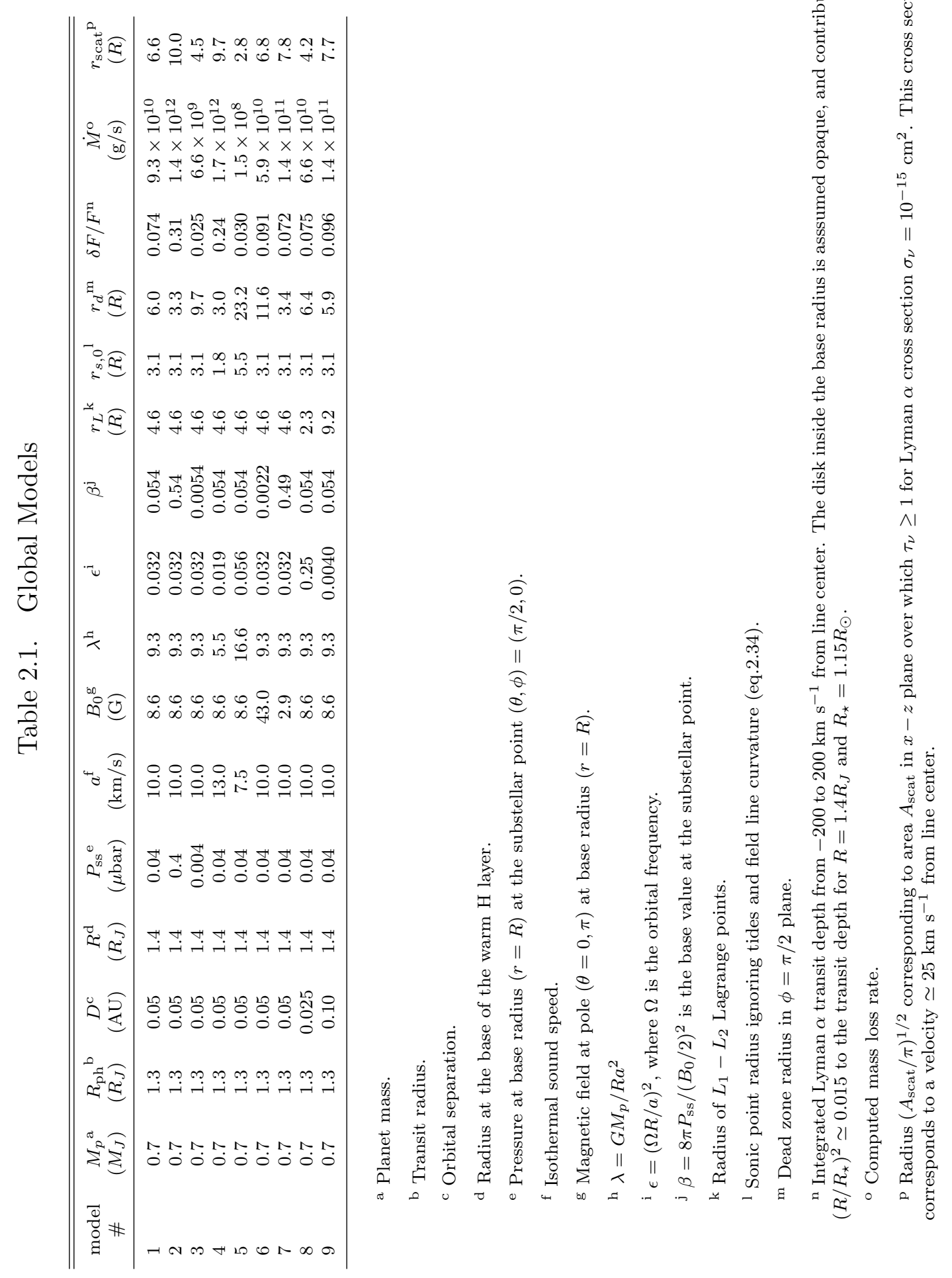


The numerical implementation of the sonic point solver deserves further discussion. Anywhere from zero to several solutions to Eq. 2.30 may be found in the interval $R \leq r \leq r_{\max }$. Some sonic points may be spurious if a potential barrier exterior to the sonic point decelerates the flow to subsonic, even zero, speed. These spurious solutions are discarded by defining the true sonic point solution to be a global minimum of $-\ln \left(B / B_{0}\right)-U / a^{2}$ which occurs within the interval $R<r<r_{\max }$. If the global minimum occurs at either of the endpoints of the interval, then there is no good sonic point solution.

For minimum at the base $r=R$, the integration is flagged as a region of parameter space with no solution, as we should have used a base position deeper in the planet. For sonic points sufficiently deep in the planet that ram pressure dominates magnetic pressure at the sonic point, we expect the Roche lobe overflow model to be recovered. The other problem is that the global minimum can occur at the outer boundary of the integration, $r=r_{\max }$. For instance, this can occur in the polar regions due to the upwardly increasing potential. For $r>r_{d}$, field lines with $\sin \theta>\sin \theta_{\text {crit }}=f^{-1 / 2}$ have outward tidal force. Field lines with outward tidal force will have fluid accelerated outward, promoting the existence of a sonic point. The field line starting at base co-latitude $\sin \theta_{b, c r i t}=\left(R / f r_{d}\right)^{1 / 2}$ will be the last field line on which the tidal force points outward. Accordingly, if no sonic point solution is found in the radial range $R<r<r_{\max }$, and the field line has $\sin \theta<\sin \theta_{b, c r i t}$, then we treat the field line as hydrostatic and set the velocity to zero. Such field lines have outwardly increasing potential, and hence outwardly decreasing density. If, however, we had used a field line model that allowed the field at $r>r_{d}$ to bend downward toward the orbital plane, it is likely that a sonic point could have been found. This affects our later numerical results, as we analytically predicted the critical tidal strength to be $\epsilon=4 / \lambda^{2}$ in order 
to find sonic points in the polar region, whereas the above prescription would force these field lines to be hydrostatic due to the potential barrier. This approximate treatment of the polar regions likely does not affect either the total mass loss rate, or the column density profiles, since the sonic point will be so far from the planet that the velocity near the planet is quite subsonic, and the fluid will be nearly hydrostatic.

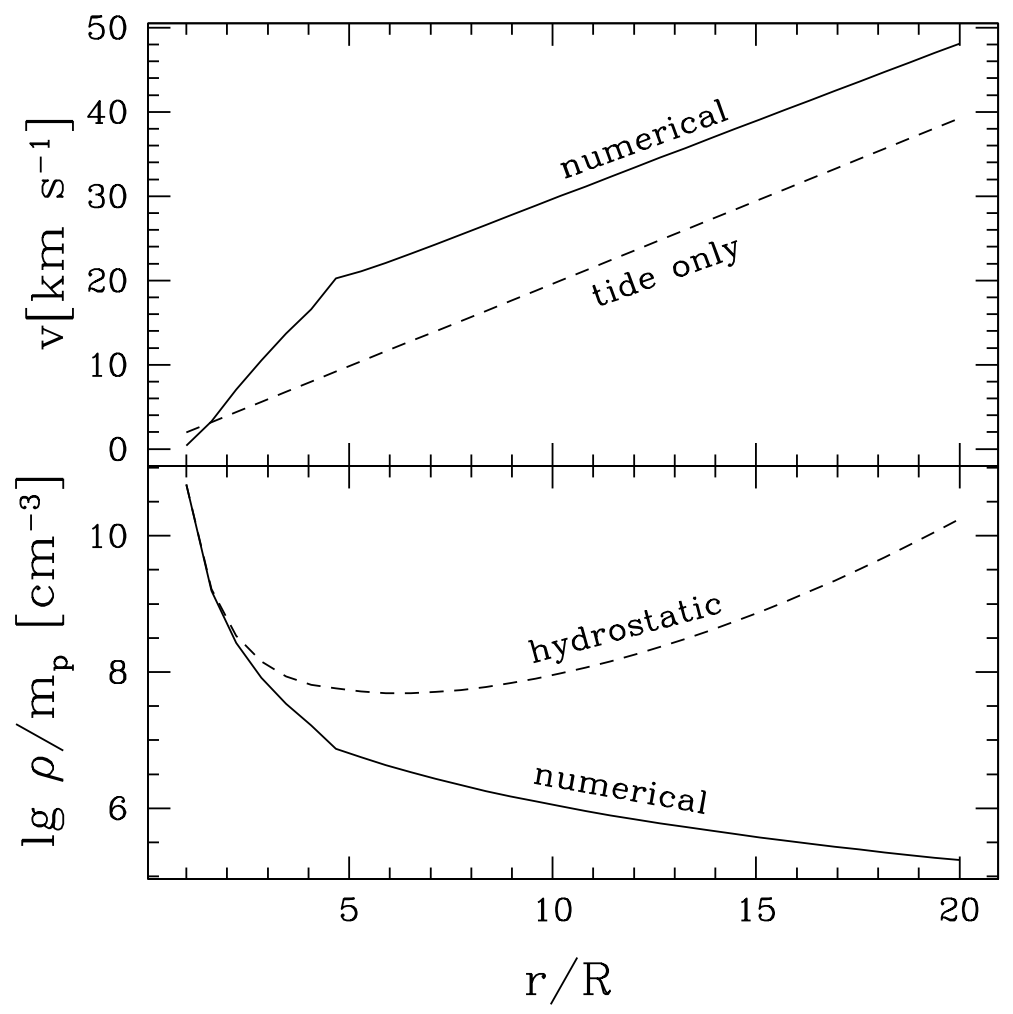

Fig. 2.10. - Velocity and density versus radius along a field line in the wind zone. Model 1 parameters from Table 2.1 are used. The field line is located at $\phi=0$ with $\theta_{b}=0.35 \mathrm{rad}$. The dead zone is at $\theta_{d}=0.48 \mathrm{rad}$ and the critical field line is at $\theta_{\mathrm{b}, \mathrm{crit}}=0.23$. The discontinuity at $r / R=4.7$ is the dead zone radius, where the field changes shape. The line labeled "tide only" is the asymptotic approximation in Eq. 2.39, and the line labeled "hydrostatic" evaluates the density using the hydrostatic balance approximation in Eq. 2.19.

Figure 2.10 shows the density and speed along a particular field line in the wind zone. The change in slope near $r=4.7 R$ is the change in field geometry at $r=$ $r_{d}$. The speed along the field line is somewhat larger than the asymptotic result in 
Eq. 2.40 due to the enthalpy $(\ln )$ terms in Eq. 2.39. In the lower panel, the density is approximately hydrostatic inside the sonic point at $r_{s}=2.4 R$, and decreases roughly as $\rho \propto B / v \propto r^{-3}$ outside that point. This plot explicitly demonstrates that the gas density in the wind zone can be orders of magnitude smaller than nearby gas in the dead zone, which satisfies hydrostatic balance.

Figure 2.12 shows contours of mass density on slices through the center of the planet in the $y-z$ plane, as viewed during transit, and the $x-z$ plane, as viewed midway between primary and secondary transit. The quantity plotted is $\rho / m_{p}=n_{\mathrm{H}}+$ $n_{p}$ (note that this quantity is distinct from the total number density $n_{\text {tot }}=n_{\mathrm{H}}+2 n_{p}$, which depends on the details of the photoionization model). Model 1 parameters listed in Table 2.1 were used. Near the planet the contours are approximately spherical, since the velocities are everywhere subsonic and the tidal force is small. The bulge at the equator is the equatorial dead zone. The poles are hydrostatic as tides have shut down the wind, and the inward tidal force at the pole causes the density to decrease outward faster than at the equator.

The impact of the tidal force on the dead zone can be seen by comparing the upper and lower panels in Figure 2.12. Along the $x$-direction, the outward tidal force decreases the size of the dead zone, but the same tidal force also causes the dead zone to have higher density. Outside the sonic point, the density in the wind zone is smaller than in the neighboring dead zone, which pushes the density contours inward in the wind zone. Near the equator, outside the dead zone, the density becomes quite small, hence the pile-up of contours near the critical angle $\sin \theta_{\text {crit }}$.

In the next section, the wind models in Table 1 are used to compute the planetary mass loss rate in the wind zone. 


\subsection{Mass Loss Rates \& Spin-Down Torque}

The mass loss rate is computed by integrating $\rho v_{r}=\rho b_{r} v_{b}$ over the surface area of the wind zone at the base. Using the base density from Eq. 2.18, the mass loss rate is

$$
\begin{aligned}
\dot{M} & =R^{2} \rho_{s s} \int_{0}^{2 \pi} d \phi \int_{\text {wind zone }} d \theta_{b} \sin \theta_{b} b_{r} v_{b}\left(\frac{\rho_{b}\left(\theta_{b}, \phi\right)}{\rho_{s s}}\right) \\
& =R^{2} P_{s s} a^{-1} \mathcal{F}(\lambda, \epsilon, \beta) \\
& =4.0 \times 10^{12} \mathrm{~g} \mathrm{~s}^{-1}\left(\frac{R}{1.4 R_{J}}\right)^{2} \\
& \times\left(\frac{P_{s s}}{0.04 \mu \mathrm{bar}}\right)\left(\frac{10 \mathrm{~km} \mathrm{~s}^{-1}}{a}\right) \mathcal{F}(\lambda, \epsilon, \beta),
\end{aligned}
$$

where the dimensionless integral

$$
\mathcal{F}(\lambda, \epsilon, \beta)=8 \int_{0}^{\pi / 2} d \phi \int_{0}^{\theta_{d}} d \theta_{b} \sin \theta_{b} b_{r}\left(\frac{v_{b}}{a}\right)\left(\frac{\rho_{b}\left(\theta_{b}, \phi\right)}{\rho_{s s}}\right)
$$

The mass loss rate for fixed $M, R$ and $B_{0}$, but varying $a, D$ and $P_{s s}$ is shown in Figure 2.11. The steep decline of $\dot{M}$ with $\lambda$ is due to smaller density at the sonic point radius. The mass loss decreases slightly for large $\epsilon$ due to the smaller fraction of open field lines.

Why is the mass loss rate $\dot{M}$ proportional to the base pressure $P_{s s}$ ? Recall that we are approximating the true atmosphere with an isothermal model. The appropriate values of $a^{2}$ and $P_{s s}$, as determined by photoionization equilibrium and heating/cooling balance, have been discussed in section 2.8. The sonic point lies at a fixed radius, given roughly by Eq. 2.34 based on the choice of sound speed $a$. The location of the base of the isothermal layer is also at a fixed radius, estimated to be $1.1 R_{\mathrm{ph}}$ (see $\S 2.4)$. By Eq. 2.19, $\rho\left(r=r_{s}\right) \propto \rho_{s s}$, therefore the density at the sonic point is 
proportional to the base density $\rho_{s s}$ (and therefore also $P_{s s}$ ). Consequently, if a larger value of $P_{s s}$ is required to explain the transit depth, the mass loss must be increased proportionally.

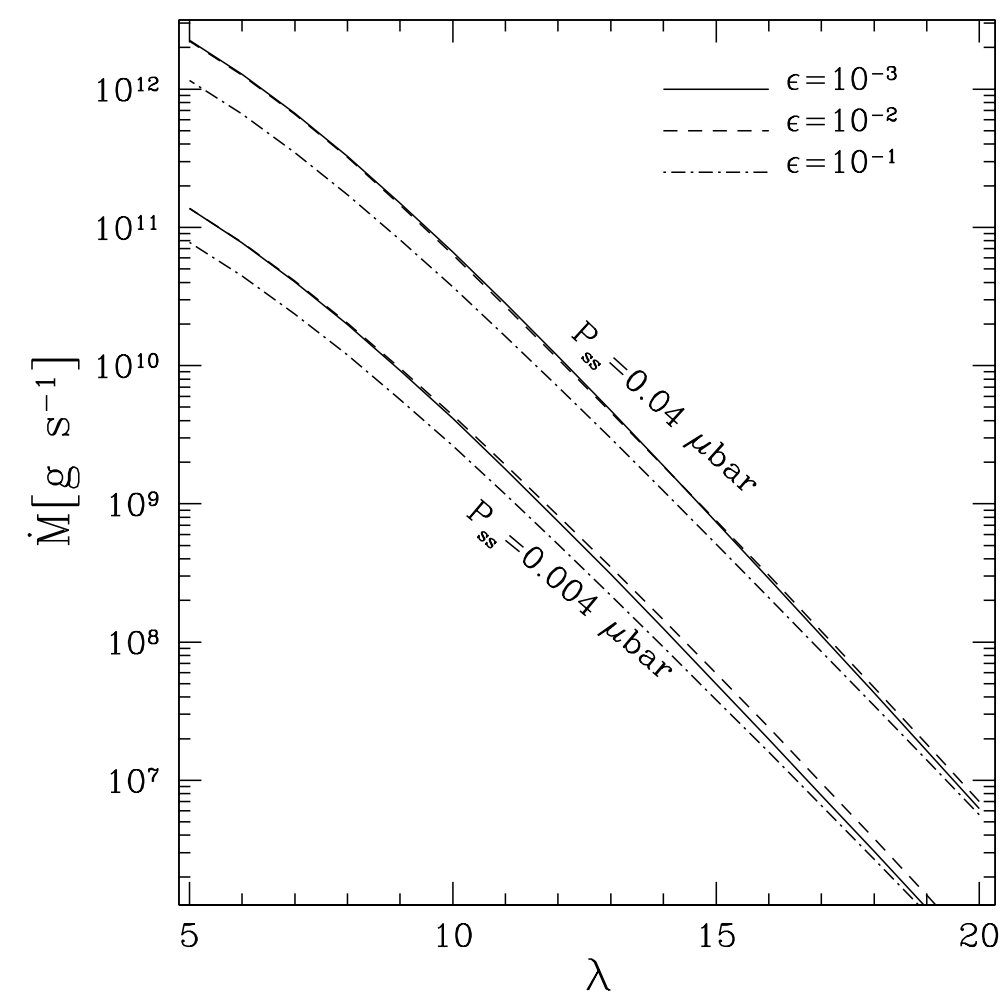

Fig. 2.11. - Total mass loss rate as a function of $\lambda$ for $M_{p}=0.7 M_{J}, R=1.4 R_{J}$ and $B_{0}=8.6 \mathrm{G}$. The upper (lower) set of three curves uses base pressure $P_{\mathrm{ss}}=$ $0.04(0.004) \mu$ bar. The line style in each set of three curves gives the value of $\epsilon$, the tidal strength.

The mass loss rates in Figure 2.11 are largely consistent with previous studies (e.g., Murray-Clay et al. 2009) when comparable gas density is used. By comparison, an unmagnetized, spherically symmetric, isothermal wind would have (Lamers \& Cassinelli 1999) $\mathcal{F} \simeq \pi \lambda^{2} \exp (3 / 2-\lambda)$, which would be a factor of $\simeq 3-10$ larger than the curves in Figure 2.11, and with a slightly flatter slope. Inclusion of the magnetic 
field decreases the mass loss rate, mainly due to the decrease in area occupied by the wind zone.

The angular momentum loss rate depends on the radius at which the torque is applied. For an isolated planet, the field lines remain rigid out to the Alfvén radius. But this location may be at many tens of planetary radii, and may be pre-empted by the interaction of the planetary wind with the stellar wind. By assuming the torque

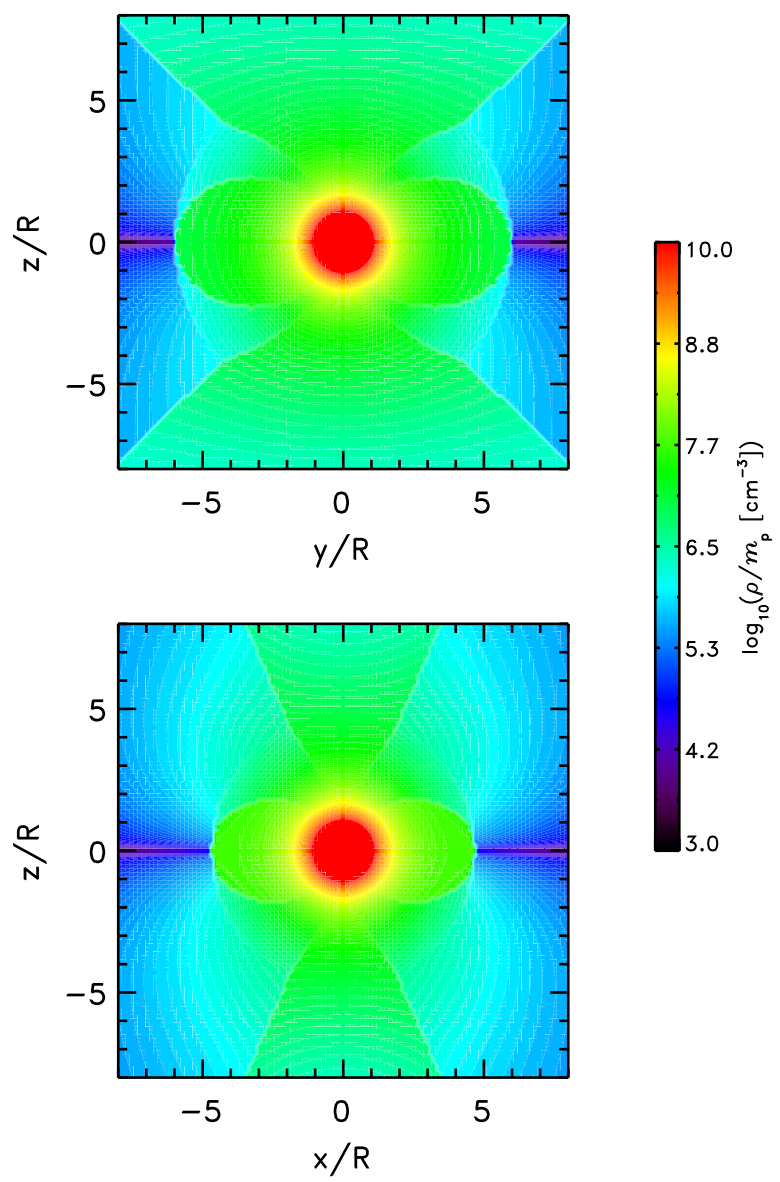

Fig. 2.12. - Contours of mass density, $\log _{10} \rho / m_{p}\left[\mathrm{~cm}^{-3}\right]$ in the $y-z$ plane (upper) at $x=0$, and in the $x-z$ plane (lower) for $y=0$. Model 1 parameters are used (see Table 2.1). 
is exerted at a radius $r_{\text {torque }}$ we estimate an angular momentum loss rate

$$
\begin{aligned}
\dot{M} \Omega r_{\text {torque }}^{2} & \simeq 7.3 \times 10^{28} \mathrm{erg}\left(\frac{1 \text { day }}{P_{\text {orb }}}\right) \\
& \times\left(\frac{r_{\text {torque }}}{10^{11} \mathrm{~cm}}\right)^{2}\left(\frac{\dot{M}}{10^{11} \mathrm{~g} \mathrm{~s}^{-1}}\right)
\end{aligned}
$$

While this torque may cause moderate changes in the spin rate for an isolated planet on Gyr timescales, it likely not large enough to torque the planet away from synchronous rotation to the extent that significant gravitational tidal heating will occur (see Arras \& Socrates 2009, for a discussion of the necessary torques).

\subsection{Neutral H Column Densities}

Given the global MHD models derived in section 2.9, we require a model for the run of ionization in the magnetosphere in order to compute observable quantities such as the transmission spectrum. In section 2.8, we discussed a model including only the dominant processes: photoionization and radiative recombination of hydrogen. In the remainder of the paper, we use the simpler optically thin limit to evaluate $n_{H}$ given $\rho:$

$$
n_{H}=\left(\frac{\sqrt{n_{\mathrm{eq}, 0}+4 \frac{\rho}{m_{p}}}-\sqrt{n_{\mathrm{eq}, 0}}}{2}\right)^{2} .
$$

Here $n_{\mathrm{eq}, 0}=J_{0} / \alpha_{B}$ is the density at which $n_{H}=n_{p}$ in the optically thin limit. The use of $J_{0}$ instead of $J$ simplifies the calculation, as only the local density is required, and not the column $N_{H}$. This approximation may underestimate $n_{H}$ near the $\mathrm{H}-\mathrm{H}^{+}$ transition, but should be adequate for our purposes. 
To evaluate the neutral hydrogen column density, we first evaluate $\rho$ ( $\S 2.9$ ), and then $n_{H}$ (Eq. 2.56), on a grid of $(x, y, z)$, with each coordinate in the range $\left(-1.1 R_{\star}, 1.1 R_{\star}\right)$. The column is displayed as seen at transit, i.e. we integrate over the coordinate along the star planet line to get column

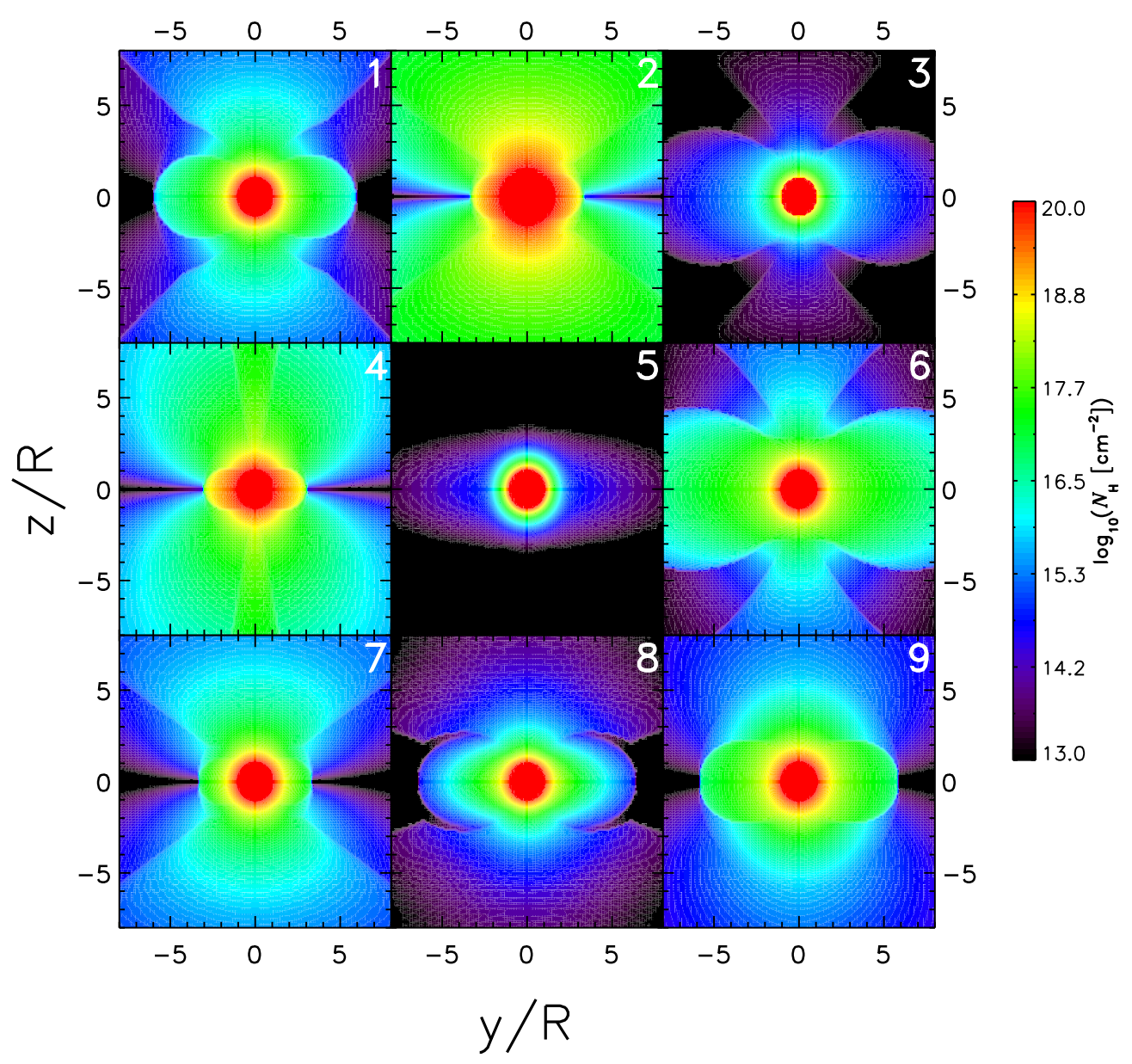

Fig. 2.13. - Contours of hydrogen column density, $\log _{10}\left(N_{H}\left[\mathrm{~cm}^{-2}\right]\right)$, versus impact parameter in the $y-z$ plane observed during transit. The parameters for each plot are matched to the labeled model numbers in Table 2.1.

$$
N_{H}(y, z)=\int_{-1.1 R_{\star}}^{1.1 R_{\star}} d x n_{H}(x, y, z)
$$

as a function of the impact parameters $y$ and $z$. Figure 2.13 shows contours of 
hydrogen column density for the 9 models listed in Table 2.1. All models have $M_{p}=$ $0.7 M_{J}$ and $R=1.4 R_{J}$, and vary a single parameter $P_{\mathrm{ss}}, a, B_{0}$ and $D$ in turn. The fiducial case, Model 1, clearly shows the equatorial and polar dead zones, as well as the mid-latitude wind zone with comparatively smaller $N_{H}$. Model 2 (3) has $P_{s s}$ larger (smaller) by a factor of 10. This has the effect of decreasing (increasing) the dead zone size as well as scaling up (down) the density in the dead zone. Model 4 (5) has larger (smaller) $a$, leading to larger (smaller) density at a given distance from the planet, as well as increasing (decreasing) the size of the dead zone. Model 6 (7) has larger (smaller) $B_{0}$, which increases (decreases) the size of the dead zone. Model 8 (9) has smaller (larger) D. Larger tide is more effective in shutting down the wind at the pole, but also decreases the size of the dead zone.

Aside from the overall magnitude mainly set by the base pressure $P_{s s}$ and sound speed $a$, the dominant parameters determining the appearance of each plot are the equatorial and polar dead zone sizes. The equatorial dead zone size (see Figure 2.4) depends on $\lambda, \beta$ and $\epsilon$. The size of the polar dead zone is set by the strength of the tidal force. The critical tidal strength in Eq. 2.37 refers to shutting down the wind at $\theta_{b}=0$, and assumes dipole field geometry. For $\epsilon>\epsilon_{\text {crit }}$, a range of $\theta_{b}$ near the pole can have the wind shut off. The result depends on the which field geometry is chosen. For instance, the sonic point Eq. 2.32 can be rederived for radial field lines. The discriminant of this cubic equation can be used to show that no sonic point can be found for

$$
\sin ^{2} \theta \lesssim \frac{1}{f \epsilon}\left(\epsilon-\frac{32}{27 \lambda^{2}}\right)
$$

The critical tidal strength for radial field lines is $\epsilon_{\text {crit }}=32 /\left(27 \lambda^{2}\right)$, a slightly different numerical coefficient than the dipole case. As $\epsilon$ increases above $\epsilon_{\text {crit }}$, the size of the 
polar dead zone increases. In the limit $\epsilon \gg \epsilon_{\text {crit }}$, the wind is shut down in the entire region $\sin ^{2} \theta \leq 1 / f$ where the tidal force is inward. For large $\epsilon$ and small $\beta$, the polar and equatorial dead zones can dominate the volume near the planet (e.g., Model 6).

\subsection{Lyman $\alpha$ Transmission Spectra}

In section 2.11 we focused on understanding the hydrogen column as a function of impact parameter, including the dependence on unknown parameters such as temperature and magnetic field. An additional effect on the transmission spectrum is the velocity gradients in the wind, which were studied in sections 2.7 and 2.9. In this section we compute the Lyman $\alpha$ transmission spectra for the global models, including both column and velocity gradient effects.

The transmission function, $T_{\nu}$, is the fraction of stellar flux at frequency $\nu$ which passes through the planet's atmosphere without suffering scattering out of the beam. In terms of the out-of-transit stellar flux, $F_{\nu}^{(0)}$, and the in-transit flux, $F_{\nu}, T_{\nu}$ is defined as

$$
T_{\nu}=\frac{F_{\nu}}{F_{\nu}^{(0)}}
$$

If the interstellar medium (ISM) optical depth, $\tau_{\nu}^{(\mathrm{ISM})}$, is constant over the stellar disk, and in time, and the geocoronal emission is independent of time, then the ratio in Eq. 2.59 depends solely on the properties of the planetary atmosphere, and is the fundamental quantity to compare to the data.

We compute $T_{\nu}$ as follows. Let $\sigma_{\nu}$ be the Lyman $\alpha$ line cross section. We simplify the problem by assuming the planet to be at the center of the stellar disk. The optical 
depth through the planet's atmosphere at position $(y, z)$ on the stellar disk is

$$
\tau_{\nu}^{(\mathrm{p})}(y, z)=\int d x n_{H}(x, y, z) \sigma_{\nu}(x, y, z)
$$

Assuming the stellar intensity is uniform over the disk,

$$
T_{\nu}=\frac{1}{\pi R_{\star}^{2}} \int d y d z e^{-\tau_{\nu}^{(\mathrm{p})}(y, z)}
$$

where the integral extends over $y^{2}+z^{2} \leq R_{\star}^{2}$. As an integrated measure of the transit depth, we compute

$$
\frac{\delta F}{F}=\frac{\int d \nu I_{\nu}^{(\star)} e^{-\tau_{\nu}^{(\mathrm{ISM})}}\left(1-T_{\nu}\right)}{\int d \nu I_{\nu}^{(\star)} e^{-\tau_{\nu}^{(\mathrm{ISM})}}}
$$

where $I_{\nu}^{(\star)}$ and $\tau_{\nu}^{(\mathrm{ISM})}$ are the unabsorbed stellar intensity and ISM optical depth, both assumed uniform over the disk. In practice, we follow ?) and integrate over $-200 \mathrm{~km} \mathrm{~s}^{-1} \leq \Delta \mathrm{v} \leq 200 \mathrm{~km} \mathrm{~s}^{-1}$. The interstellar medium (ISM) is assumed to have a temperature $T_{\text {ism }}=8000 \mathrm{~K}$ and hydrogen column $N_{\mathrm{H}, \text { ism }}=10^{18.4} \mathrm{~cm}^{-2}(?)$, implying the line is dark inside linewidth $\Delta v=c\left(\nu-\nu_{0}\right) / \nu_{0} \lesssim 50 \mathrm{~km} \mathrm{~s}^{-1}$. For $I_{\nu}^{(\star)}$ we use the following (unnormalized) fit to the quiet solar Lyman $\alpha$ spectrum presented in ?) (downloaded from http://www.mps.mpg.de/projects/soho/sumer/FILE/ Atlas.html):

$$
I_{\nu}^{(*)}=\left[1+\left(\frac{|\Delta v|}{67 \mathrm{~km} \mathrm{~s}^{-1}}\right)^{3}\right]^{-1}
$$


The Voigt function $H(a, u)(?)$ is used for the line profile, giving

$$
\sigma_{\nu}=\frac{\pi e^{2}}{m_{e} c} f_{12} \frac{1}{\sqrt{\pi} \Delta \nu_{D}} H\left(a_{D}, u\right)
$$

where $-e$ is the electron charge, $m_{e}$ is the electron mass, and $f_{12}=0.42, \lambda_{0}=$ $1215 \AA$ and $\nu_{0}=c / \lambda_{0}$ are the Lyman $\alpha$ oscillator strength, line center wavelength and frequency. The Doppler width is $\Delta \nu_{D}=\nu_{0} v_{\mathrm{th}} / c$ where the hydrogen atom thermal velocity is $v_{\mathrm{th}}=\left(2 k_{b} T / m_{p}\right)^{1 / 2}$. The damping parameter is $a_{D}=\Gamma / 4 \pi \Delta \nu_{D}$, where the natural linewidth is $\Gamma=6.25 \times 10^{8} \mathrm{~s}^{-1}$. Finally, the distance from line center, in Doppler widths, including both bulk motion and thermal broadening, is

$$
u=\frac{\nu-\nu_{0}}{\Delta \nu_{D}}+\frac{v_{x}}{v_{\mathrm{th}}}
$$

where $v_{x}$ is the bulk motion directed from planet toward star, which is away from the observer.

There are three instructive limits of Eq. 2.61 to guide the intuition. First, if $v_{x}=0$ and the gas is optically thick over an area $A_{\text {tran }}$, with negligible optical thickness outside this area, the fraction of flux absorbed by the planet is

$$
1-T_{\nu}=\frac{A_{\text {tran }}}{\pi R_{\star}^{2}}=0.013\left(\frac{A_{\text {tran }}}{\pi\left(1.3 R_{J}\right)^{2}}\right)\left(\frac{1.15 R_{\odot}}{R_{\star}}\right)^{2} .
$$

Next, if $v_{x}=0$ and the gas is optically thin, then the transit signal due to the optically thin area is

$$
1-T_{\nu} \simeq \frac{1}{\pi R_{\star}^{2}} \int_{\tau_{\nu}^{(\mathrm{p})} \ll 1} d y d z \tau_{\nu}^{(\mathrm{p})}(y, z) \equiv\left\langle\tau_{\nu}^{(\mathrm{p})}\right\rangle
$$

which is just the area-averaged optical depth, and is proportional to the total number 


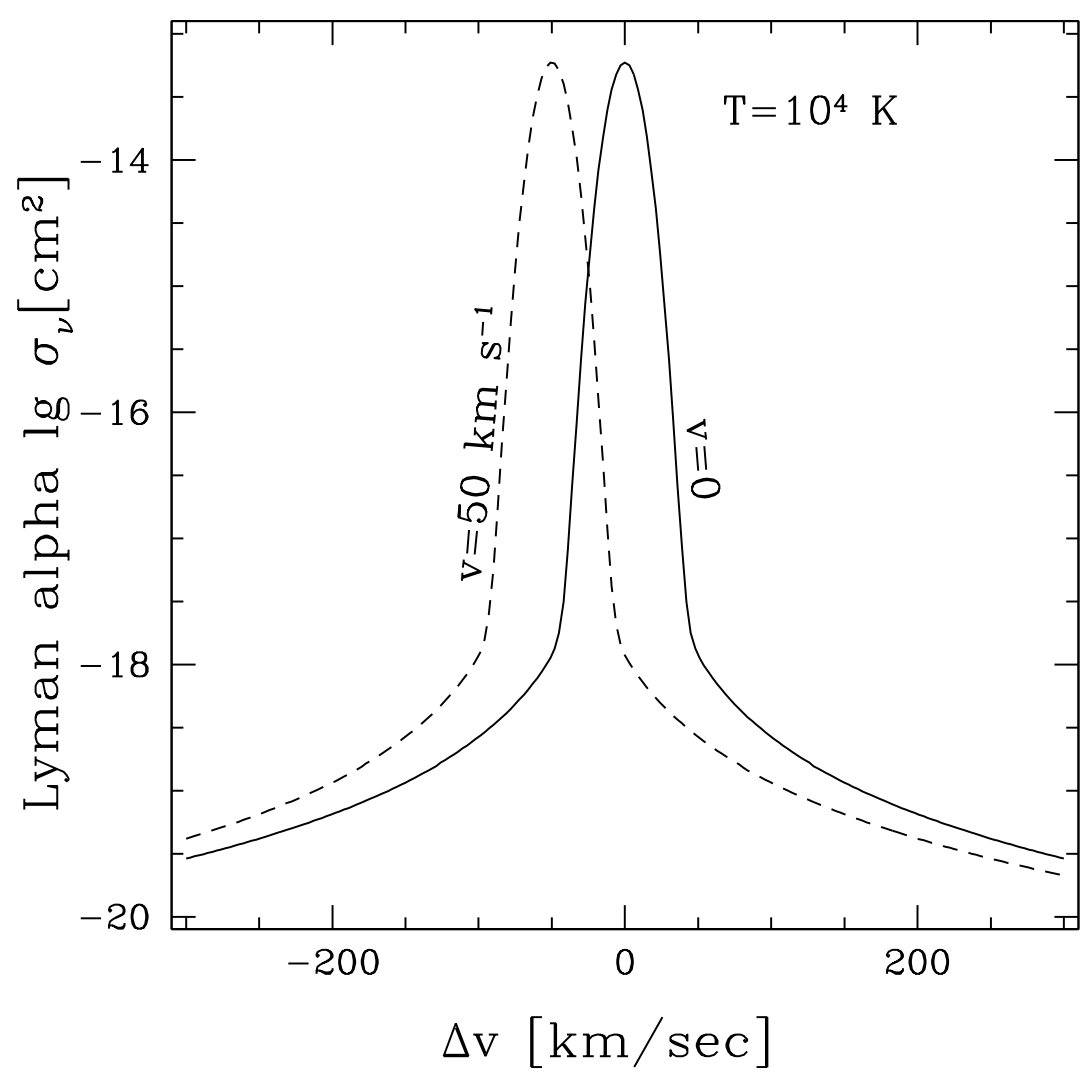

Fig. 2.14. - Lyman $\alpha$ cross section as a function of velocity width from line center. Both profiles are given for a temperature $T=10^{4} \mathrm{~K}$. The solid line is for zero bulk velocity away from the observer, while the dashed line is for $\Delta v=50 \mathrm{~km} \mathrm{~s}^{-1}$. For bulk velocity toward the observer, the dashed curve would be reflected about $\Delta v=0$.

of hydrogen atoms times their mean cross section. The third limit is when thermal motions are much smaller than bulk motions, and the line profile can be approximated as a delta function $\delta\left[\nu-\nu_{0}\left(1-v_{x} / c\right)\right]$. In this case, the cross section is only nonzero at those values of $x_{\star}=x_{\star}(\nu, y, z)$ where $v_{x}\left(x_{*}, y, z\right)=c\left(\nu_{0}-\nu\right) / \nu_{0}$ is satisfied, so that the photon is shifted to line center in the atom's frame. In this case the optical depth 
becomes

$$
\begin{gathered}
\tau_{\nu}^{(\mathrm{p})}(y, z)=n_{H}\left(x_{\star}, y, z\right) \frac{\pi e^{2}}{m_{e} c} f_{12} \frac{\lambda_{0}}{\left|\partial v_{x}\left(x_{\star}, y, z\right) / \partial x\right|} \\
\simeq 2 \times 10^{-3}\left(\frac{n_{H}\left(x_{\star}, y, z\right)}{1 \mathrm{~cm}^{-3}}\right)\left(\frac{P_{\mathrm{orb}}}{1 \text { day }}\right) \frac{\Omega}{\left|\partial v_{x}\left(x_{\star}, y, z\right) / \partial x\right|},
\end{gathered}
$$

where in the second equality we have scaled the velocity gradient to the orbital frequency $\Omega$. Eq.2.68 shows that for hydrogen densities $n_{H} \gtrsim 10^{2-3} \mathrm{~cm}^{-3}$, the optical depth along a line of sight will be high provided that there is gas with sufficiently large velocity to absorb at that wavelength.

Figure 2.14 shows the cross section as a function of frequency in velocity units, at $T=10^{4} \mathrm{~K}$ and for $\Delta v=0$ and $\Delta v=50 \mathrm{~km} \mathrm{~s}^{-1}$. For HD 209458b, the transit radius is $R_{\mathrm{ph}}=1.3 R_{J}$ and the stellar radius is $R_{\star}=1.15 R_{\odot}$, giving a transit depth $\delta F / F=0.013$ in the optical continuum. To explain the line-integrated Lyman $\alpha$ transit depth $\simeq 9 \%$ (e.g., see the discussion in Ben-Jaffel 2008) one could invoke an opaque disk of area $\sim \pi\left(2.6 R_{\mathrm{ph}}\right)^{2}$. The central issue is that this disk must be opaque at $\Delta v \gtrsim \pm 100 \mathrm{~km} \mathrm{~s}^{-1}$ from line center, requiring large columns of neutral hydrogen at radii $2-3 R_{\mathrm{ph}}$.

In Figure 2.9, triangle symbols show where Lyman $\alpha$ radiation at frequencies $\pm 100 \mathrm{~km} \mathrm{~s}^{-1}$ from line center is optically thick on a radial line outward. This point is much deeper in the atmosphere from where Lyman continuum at threshold becomes optically thick, due to the rapid decrease in Lyman $\alpha$ cross section. Clearly in order to model the transit spectrum in the wavelength region of interest, one must include regions down to $\sim 1-10$ nbar in the atmosphere. To quantify this statement, we compute the optical depth through the H layer where $n_{H} \simeq \rho / m_{p}$ is given by Eq. 2.17. Assuming the dominant contribution arises from the layer of steeply falling density, 
the slant optical depth is dominated by the region near $x=0$ and we find

$$
\begin{aligned}
& \tau_{\nu}^{(\mathrm{p})}(y, z)=\sigma_{\nu}\left(\frac{\rho(x=0, y, z)}{m_{p}}\right) \\
\times & \int d x \exp \left[-\frac{1}{2 a^{2}}\left(\frac{G M_{p}}{b^{3}}-3 \Omega^{2}\right) x^{2}\right] \\
\simeq & \sigma_{\nu}\left(\frac{\rho(x=0, y, z)}{m_{p}}\right)\left(\frac{2 \pi b^{3} / \lambda R}{1-\left(b / r_{L}\right)^{3}}\right)^{1 / 2} \\
\simeq & 1.2\left(\frac{100 \mathrm{~km} \mathrm{~s}^{-1}}{\Delta v}\right)^{2}\left(\frac{P(x=0, y, z)}{1 \mathrm{nbar}}\right)\left(\frac{10 \mathrm{~km} \mathrm{~s}^{-1}}{a}\right)^{2} \\
\times & \left(\frac{10}{\lambda}\right)^{1 / 2}\left(\frac{b}{R}\right)^{3 / 2}\left(\frac{R}{1.3 R_{J}}\right)\left(1-\left(b / r_{L}\right)^{3}\right)^{-1 / 2} .
\end{aligned}
$$

Here $b=\sqrt{y^{2}+z^{2}}$ is the impact parameter, Eq. 4.10 was used for the tidal potential, $r_{L}$ is given by Eq. 2.7, and the last equality assumes the cross section is on the damping wing (see Figure 2.14). In the $\mathrm{H}^{+}$layer, Eq. 2.69 should be multiplied by $1 / 2$ to account for the smaller $\mathrm{H}$ atom scale height. Eq.2.69 agrees roughly with the position of the triangles in Figure 2.9, keeping in mind that the slant length is a factor of a few larger than the scale height. Eq.2.69 shows that the Lyman $\alpha$ transmission spectrum at $\Delta v= \pm 100 \mathrm{~km} \mathrm{~s}^{-1}$ is probing down to $\lesssim$ nbar pressures, depending on the value of $b / R$.

To give a more precise numerical estimate of the transit depth, we first compute the integrated quantity $\delta F / F$ as in Eq. 2.62 for the 9 models in Table 2.1. The result is given in the table. The velocity range is taken to be $-200 \leq \Delta v\left[\mathrm{~km} \mathrm{~s}^{-1}\right] \leq 200$. Since $1-T_{\nu}$ decreases away from line center, $\left(1-T_{\nu}\right) F_{\nu}^{(0)}$ is peaked somewhat closer to line center than $F_{\nu}^{(0)}$, the amount depending on the details of the atmosphere. Transit depths of the correct magnitude $\delta F / F \sim 5-10 \%$ can be achieved by adjusting the main parameters, $P_{\mathrm{ss}} \simeq 10-100$ nbar and $a \simeq 8-12 \mathrm{~km} \mathrm{~s}^{-1}$ to have values as expected from the $1 \mathrm{D}$ model in Figure 2.9. The parameters $B_{0}$ and $D$ have a lesser 


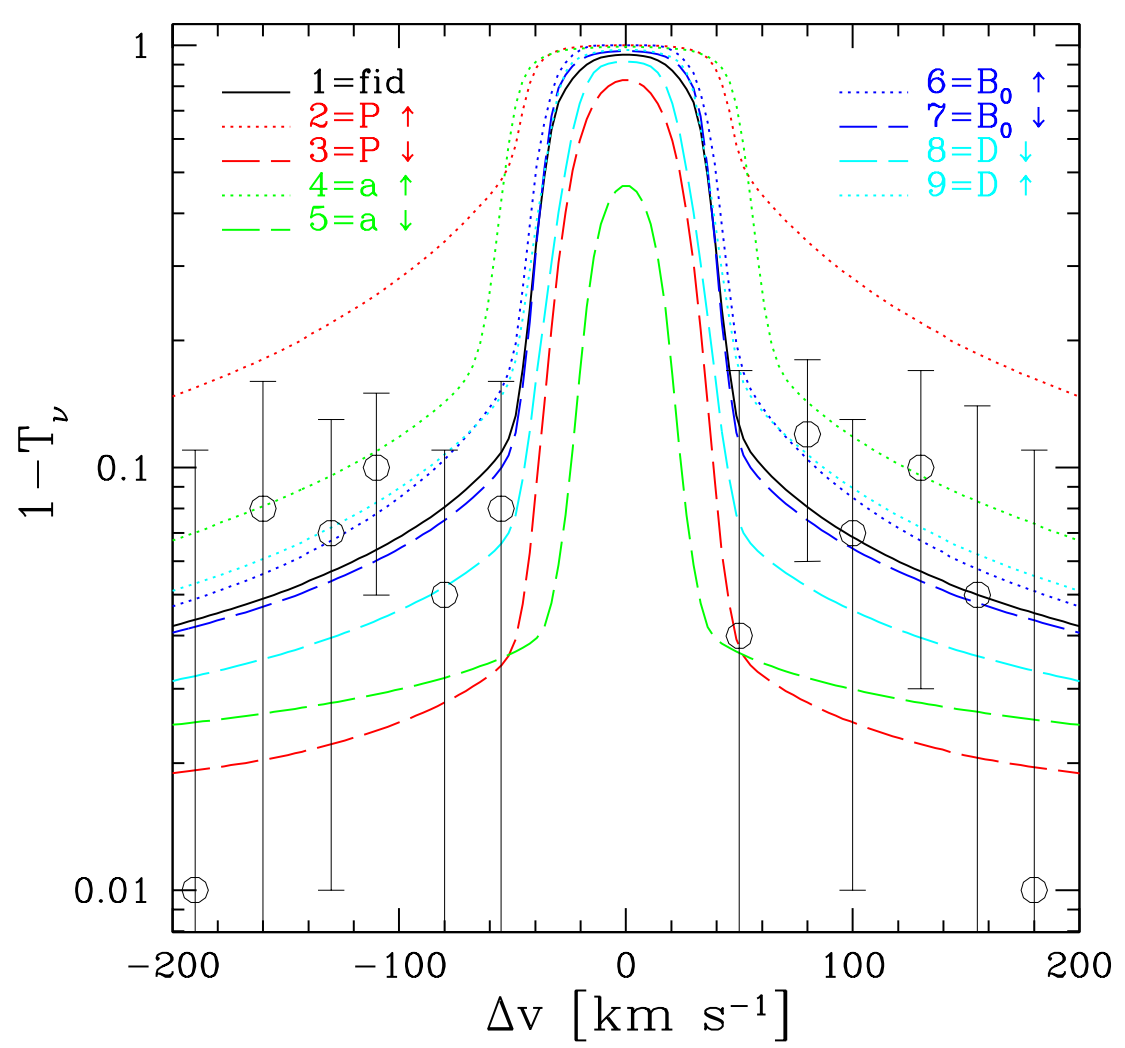

Fig. 2.15.- Fractional flux decrease, $1-T_{\nu}$, versus frequency in velocity units for HD 209458b. Curves for Models 1-9 from Table 2.1 are computed from Eq. 2.61 and points with error bars are the data from Figure 6 of ?).

impact by comparison.

The frequency dependent transit depth, $1-T_{\nu}$, was computed as in Eq. 2.61 for the 9 models listed in Table 2.1, and compared to the data for $\left(F_{\nu}^{(0)}-F_{\nu}\right) / F_{\nu}^{(0)}$ from Figure 6 of Ben-Jaffel (2008). The results are shown in Figure 2.15. Near line center, nearly the entire planetary atmosphere is optically thick, and absorption is nearly complete. Moving out from line center in the Doppler core, the cross section eventually becomes small enough that part of the atmosphere becomes optically thin, after which $1-T_{\nu}$ decreases rapidly. The curves level out when the damping wing is reached, after which $1-T_{\nu}$ decreases slowly as the $\tau_{\nu}^{(\mathrm{p})}=1$ point moves deeper into 
the atmosphere as $P(x=0, y, z) \propto \Delta v^{2}$.

Given the large error bars, a range of parameter space agrees with the data if the warm H layer extends sufficiently deep. For instance, Model 3 with base pressure $P_{s s}=4$ nbar is well below the data points with the smallest error bars, in agreement with Murray-Clay et al. (2009). The most sensitive parameter dependencies are with the base pressure, $P_{\mathrm{ss}}$, and the sound speed (temperature), a. Increasing the magnetic field has the effect of increasing $1-T_{\nu}$ due to larger $N_{H}$ in the magnetosphere. Somewhat offsetting this effect is that increasing $B_{0}$ decreases the size of the wind zone, which decreases absorption near line center due to velocity gradients. Perhaps counter-intuitively, moving the planet further from the star increases the transit depth. Inspection of Figure 2.9 shows that the $\mathrm{H}$ extends to both lower pressure and larger radius for more distant planets with atmospheres in photoionization equilibrium. Lastly, we note that velocity gradients are only important for $\Delta v \lesssim 50 \mathrm{~km} \mathrm{~s}^{-1}$, and are more important for smaller $D$ due to the larger tidal force.

We end this section with a brief discussion of scattering of Lyman $\alpha$ from $\mathrm{H}$ atoms in the magnetosphere. The problem with observing Lyman $\alpha$ during transit is that large $N_{H}$ is required to create $\tau_{\nu}^{(\mathrm{p})} \simeq 1$ at $\Delta v \gtrsim 100 \mathrm{~km} \mathrm{~s}^{-1}$. By contrast, at line center the cross section is $\sim 10^{5}$ times larger, implying the atmosphere is optically thick at line center out to much larger radii. We suggest that scattering of stellar Lyman $\alpha$ during the orbital phases in which the planet is moving toward or away from the observer may be detectable, and provides a probe of thermal gas in the magnetosphere, complementary to the transmission spectrum measured during transit. During the orbit, the Doppler shift of the scattered spectrum varies in time due to the variation in line-of-sight orbital motion. The orbital velocities naturally produces a feature in the spectrum well outside the line core where ISM absorption 
dominates.

For a planet in circular orbit, there is no relative radial motion with respect to the star, and the stellar spectrum at the planet is not Doppler shifted. However, when an $\mathrm{H}$ atom in the planet resonantly scatters a stellar photon, that $\mathrm{H}$ atom is moving with respect to the observer due to the planet's orbital motion. Photons emitted by the star near line center $\left(\Delta v \lesssim 67 \mathrm{~km} \mathrm{~s}^{-1}\right)$ have their frequencies shifted by $v_{\text {orb }}=210 \mathrm{~km} \mathrm{~s}^{-1}\left(1 \text { day } / \mathrm{P}_{\text {orb }}\right)^{1 / 3}$ (for a solar mass star) due to the planet's orbital motion. To assess the area presented by the magnetosphere, we computed the area in the $x-z$ plane for which $\tau_{\nu}^{(\mathrm{p})} \gtrsim 1$ for $\sigma_{\nu}=10^{-15} \mathrm{~cm}^{2}$, which corresponds to $\Delta v=25 \mathrm{~km} \mathrm{~s}^{-1}$ from line center for $T=10^{4} \mathrm{~K}$. The results are tabulated in Table 2.1. We find that the effective radius of the scattering disk is $r_{\mathrm{sc}} \sim(5-10) R$ for the models shown. The scattering disk for Lyman $\alpha$ is significantly larger than the radius inferred during transit. Assuming none of the resonantly scattered Lyman $\alpha$ photons are absorbed, and also assuming the Lambert phase function (Hapke 1993) as an estimate, the reflected flux is $F_{\text {refl }}(\nu)=F_{\star}\left(\nu^{\prime}\right)(2 / 3 \pi)\left(r_{\mathrm{sc}} / D\right)^{2}$, where $\nu \simeq \nu_{0} \pm v_{\text {orb }}$ is the observed frequency, $\nu^{\prime} \simeq \nu_{0}$ was the frequency emitted by the star before Doppler shift, and $F_{\star}(\nu)$ is the stellar Lyman $\alpha$ spectrum. The size of the reflected flux relative to the flux emitted by the star out on the wing at frequency $\nu$ is then $F_{\text {refl }}(\nu) / F_{\star}(\nu)=\left(F_{\star}\left(\nu^{\prime}\right) / F_{\star}(\nu)\right)(2 / 3 \pi)\left(r_{\text {sc }} / D\right)^{2}$. Inspection of Eq. 4.27 shows that the line center flux is $\simeq 30\left(\Delta v / 200 \mathrm{~km} \mathrm{~s}^{-1}\right)^{3}$ times larger than that at $\Delta v$. This acts to enhance the scattered flux signal relative to the background flux level. Numerically we find the ratio of scattered, Doppler shifted flux to background stellar flux is then $F_{\text {refl }}(\nu) / F_{\star}(\nu) \simeq 0.4\left(r_{\text {sc }} / 10 R_{J}\right)^{2}\left(1 \text { day } / \mathrm{P}_{\text {orb }}\right)^{7 / 3}$. While this signal may be small for HD $209458 \mathrm{~b}$ at $P_{\text {orb }}=3.5$ days, $F_{\text {refl }}(\nu) / F_{\star}(\nu) \simeq 0.02\left(r_{\mathrm{sc}} / 10 R_{J}\right)^{2}$, for planets with $P_{\text {orb }}=1-2$ days it may be large enough to be observable. 


\subsection{Comparison to Roche-Lobe Overflow}

The magnetic wind model developed in this paper differs in several respects from purely hydrodynamic mass loss models (e.g., Lubow \& Shu 1975). In the standard Roche-lobe model for nearly equal mass stars, nearly all the gas leaves the donor in a narrow, cold stream through the L1 Lagrange point. The first assumption underlying this solution is that $r_{s, 0} \gg r_{\mathrm{L} 1}$, so that the gas is subsonic at the L1 equipotential for most $(\theta, \phi)$. From Eq. 2.7 and Eq. 2.34, this ratio is $r_{s, 0} / r_{L}=\left(\epsilon \lambda^{2} / 9\right)^{1 / 3}$, and hydrodynamic Roche lobe overflow requires $\epsilon \gg 9 / \lambda^{2}$. Figure 2.5 plots $\epsilon$ versus $\lambda$, and shows that most, but not all, transiting planets are indeed in the $r_{s, 0} \gg r_{L}$ regime; ignoring magnetic effects, Roche lobe overflow would then be a good approximation. In the opposite limit of $\epsilon \ll 9 / \lambda^{2}$, the solution would more closely resemble a thermally driven wind weakly perturbed by tides. The second assumption underlying a narrow flow through L1 is that the mass ratio of the two bodies is near unity. Although the tidal expansion $r \ll D$ in Eq. 4.10 ignores the difference in potential between the L1 and L2 Lagrange points, inclusion of higher order terms gives $U_{L 2}-U_{L 1} \simeq$ $2 G M_{p} /(3 D)$ for the potential difference (Murray \& Dermott 2000). When the ratio $2 G M_{p} /\left(3 D a^{2}\right)=(2 / 3)\left(\epsilon \lambda M_{p} / M_{\star}\right)^{1 / 3} \ll 1$, the density difference between the L1 and L2 points is small, and nearly equal mass loss is expected through L1 and L2. While mass loss through L1 enters into an orbit around the star, mass loss through L2 leads to gas in a circumbinary orbit.

MHD effects, in particular the existence of a dead zone, further limit the applicability of the Roche lobe model. If the planet has a sufficiently large magnetic field that the L1 Lagrange point lies inside the dead zone, gas pressure is insufficient to open the magnetic field lines and the flow through the L1 point is expected to be choked off. Also, the magnetic field may torque the gas, keeping it in corotation with 
the planet out to the Alfvén radius. By contrast, if $B^{2} / 8 \pi \ll P \simeq \rho v^{2}$ at the sonic point, and $r_{s, 0} \gg r_{L}$, magnetic stresses and tides may be ignored the Roche-lobe model is expected to be recovered.

For the models of HD 209458b considered in this paper, inspection of Table 2.1 shows that $r_{s, 0}, r_{L}$ and the dead zone radius $r_{d}$ may be within factors of a few of each other, and the situation is more complex than the simplified Roche-lobe overflow model permits.

\subsection{Summary}

The objective of this paper was to develop a model for the upper atmospheres of hot Jupiters, including the influence of a dynamically important magnetic field. Our starting point ( $\S$ 's 2.2, 2.3, 2.4, 2.6, 2.7, 2.9 and Appendix 2.15) was to estimate field strengths for hot Jupiters, and to apply the theoretical model developed for MHD winds from stars to the case of winds escaping from the upper atmospheres of planets. In the process, we included strong tidal forces from the parent star ( $\S$ 's 2.5 , 2.6, and 2.7). We computed a 1D model of the temperature profile and ionization state of the atmosphere ( $\S 2.8$ ), and constructed maps of neutral hydrogen column and fluid velocities to understand the mass loss and transmission spectra of HD 209458b ( $\S$ 's 2.9, 2.10, 2.11, and 2.12). We contrast this model to the standard Roche-lobe overflow model ( $§ 2.13)$ and verify, a posteriori, the validity of the MHD approximation (Appendix 2.16).

In section 2.3, we discussed the application of dynamo models to understand the magnetic field strength generated by the planet, which is currently unconstrained by observations. Using the recent results of Christensen et al. (2009), which showed that the dynamo field increases with heat flux in the planet's core, we argued that the 
large radii of hot Jupiters, and hence large core flux, imply that the magnetic fields of inflated hot Jupiters may be larger than Jupiter's field. This motivated exploring a wide range of possible magnetic field strengths, both smaller and larger than Jupiter's field.

The formation of a dead zone, in which gas pressure is insufficient to open up magnetic field lines, was motivated with a toy problem $(\S 2.2)$ as intuition for understanding the detailed structure of the hydrostatic model ( $\S 2.6)$. The projection of the tidal force along magnetic field lines was used to derive the "magnetic Roche lobe radius" ( $(2.5)$, outside of which gravity points outward along the magnetic loop. Net gravity can point outward for loops slightly larger than the distance to the L1-L2 Lagrange points, even in the plane perpendicular to the star-planet line. As a result of net outward gravity, the density may increase outward, as shown in Figure 2.3. We defined the key parameters $\lambda$ and $\epsilon$, characterizing the binding energy of the gas and the strength of tides, and their values for the observed transiting planets were given in Figures 2.1 and 2.2. Many close-in planets have weakly bound atmospheres with $\lambda \lesssim 10$, and are subject to strong tidal forces with $\epsilon \gtrsim 0.1$. The magnetic field strength was characterized by the plasma $\beta$ evaluated at the base of the atmosphere. Solutions for the radius of the dead zone depend on the parameters $\lambda, \beta$ and $\epsilon$, as shown in Figure 2.4. We found that for typical parameters, the dead zone extends to $\simeq(3-20) R$, implying that much of the volume of the magnetosphere near the planet is occupied by bound gas with no bulk velocity. Even gas outside the Roche-lobe radius can be static, if the dead zone is larger than the Roche-lobe radius.

Open field lines, which are capable of supporting an outflow, were discussed in section 2.7. The momentum equation along field lines was used to compute the positions of the (slow magneto)sonic points for a set of models using dipole geometry. 
Analytic solutions in the limit of strong and weak tides were given, which illustrated that inward tidal forces at the magnetic poles (for a magnetic dipole moment aligned with the orbital angular momentum axis) may eliminate the sonic point solutions near the planet. Thus, sufficiently strong tides effectively shut off the wind, creating a second dead zone at the poles. Figures 2.6 and 2.7 show solutions for sonic point radius and base velocity versus footpoint position. When the sonic point position moves far from the planet, the base velocity becomes small, and the field lines are effectively hydrostatic. Depending on $\epsilon$ and $\beta$, the equatorial and polar dead zones may dominate the volume near the planet. Lastly, we estimated the asymptotic flow speed due to tides in Eq. 2.40, showing that $v_{\text {asymp }} \ll 100 \mathrm{~km} \mathrm{~s}^{-1}$ for the orbital periods and stellar radii of interest. Consequently, bulk motion cannot affect the Lyman $\alpha$ line profile at $\Delta v \gtrsim 100 \mathrm{~km} \mathrm{~s}^{-1}$ from line center.

As a prelude to discussion of global models of the magnetosphere, and the Lyman $\alpha$ transmission spectrum, we presented a simple spherical model of photoionization and thermal balance $(\S 2.8)$ in order to assess the size of the "warm" neutral H layer. We computed the depth dependence of photoelectric heating in Figure 2.8, showing that the heating drops off with pressure as a power-law, rather than an exponential, into the atmosphere. The resulting photoelectric heating, which we assumed was balanced by collisionally-excited Lyman $\alpha$ cooling, gives temperatures $T \simeq(5-10) \times 10^{3} \mathrm{~K}$ down to pressures $P \simeq(10-100)$ nbar. As a result, this neutral $\mathrm{H}$ layer contributes significantly to the radius, as shown in Figure 2.9. As first stressed by Koskinen et al. (2010), the location of the warm H layer is key in understanding the large observed transit depths $\delta F / F \sim 5-10 \%$. The transit depth due to the layer extending upward from the $\mathrm{H}-\mathrm{H}^{+}$ionization layer alone is too small to explain the observations of $\mathrm{HD}$ 209458b, as discussed in detail by Murray-Clay et al. (2009). 
Global models of the magnetosphere were constructed ( $\S 2.9$ ), both to compute mass loss rates $(\S 2.10)$, and to construct maps of the neutral hydrogen column densities for a range of parameters as observed during transit ( $§ 2.11$ ). A by-product of the warm, deep $\mathrm{H}$ layer is a larger mass loss rate than in studies with more shallow H layers (e.g., Murray-Clay et al. 2009). The net mass loss rates are still insufficient to evaporate the planet, and are reduced by a factor of 3-10 due to the presence of the magnetic field for the parameters used. The largest columns within a few $R$ of the planet occur in the dead zones, and may receive a contribution from $\mathrm{H}$ atoms outside the Roche lobe, but which are still bound to the planet. Hence, the observation of $\mathrm{H}$ atoms outside the Roche lobe alone cannot be stated as evidence for mass loss.

The 9 global models in Table 2.1 were used to compute Lyman $\alpha$ transmission spectra in section 2.12. We stress that the observational quantity most directly comparable with models of the magnetosphere is the fractional flux decrease between in and out of transit spectra - this quantity is relatively independent of ISM absorption, geocoronal contamination, and the background stellar spectrum, and is directly computable from atmosphere models. The comparison between the models and data for HD 209458b from Ben-Jaffel (2008) is shown in Figure 2.15. By variation of the base pressure of the warm $\mathrm{H}$ layer, and temperature, models can be made to bracket the data points, although the large error bars do not allow precise determination of the atmosphere's parameters. Increased magnetic field is shown to increase the transit depth, as does moving the planet further from the star.

A comparison of the MHD wind model presented in this paper with the more commonly-used Roche-lobe overflow model was given in section 2.13. It was argued that different regimes of accretion are possible depending on the position of the sonic point (of an isolated body), the L1-L2 Lagrange points, and the size of the dead 
zone. In particular, if the L1 Lagrange point is inside the dead zone, gas pressure is insufficient to open up the magnetic field lines, and a narrow flow through L1 is not possible. These considerations suggest that mass loss from hot Jupiters may be more complex than the simple Roche-lobe overflow model. Estimates of collision rates in the atmosphere (Appendix B) demonstrate the validity of the MHD approximation, that the e-p-H gas is well-coupled collisionally at the expected densities and temperatures in the atmosphere, and that even neutral $\mathrm{H}$ gas cannot ballistically escape the planet.

The model presented in this paper shows that magnetic fields may strongly affect theoretical estimates of fluid density and velocity in the upper atmosphere, and even the interpretation of transit depths, since neutral $\mathrm{H}$ atoms outside the Roche-lobe radius may not be escaping. In future work, we hope to include additional physical effects, such as the interaction with the stellar wind, more detailed photoionization calculations including heavy elements, and collisional (non-MHD) effects, which will allow a more comprehensive physical picture of the upper atmospheres of hot Jupiters.

\subsection{Appendix: MHD Wind Equations}

In this appendix, we present the MHD equations and discuss how currents produced in the magnetosphere modify the field produced by the planet's core. This discussion motivates our choice of field geometry used in the global models.

There is a well developed literature for axisymmetric winds from rotating, magnetized stars. An excellent review is given by Spruit (1996). Here we rely heavily on the analytic studies in Mestel (1968) and Mestel \& Spruit (1987). The inclusion of the magnetic field can greatly affect the mass loss rate and wind speed for sufficiently fast rotation. We are not aware of detailed studies of wind launching from rotating magnetized bodies including the non-axisymmetric tidal acceleration. We postpone a 
numerical study of such a problem to a future investigation, here using a semi-analytic treatment.

The three-dimensional MHD equations for steady isothermal flow in the frame corotating with the planet are mass continuity

$$
\boldsymbol{\nabla} \cdot(\rho \boldsymbol{v})=0
$$

the Euler equation

$$
\boldsymbol{v} \cdot \boldsymbol{\nabla}+2 \boldsymbol{\Omega} \times \boldsymbol{v}=-a^{2} \nabla \ln \rho-\nabla U+\frac{\boldsymbol{J} \times \boldsymbol{B}}{c \rho}
$$

Ohm's law for infinite conductivity

$$
\boldsymbol{E}=-\boldsymbol{v} \times \boldsymbol{B} / c
$$

the induction equation

$$
\boldsymbol{\nabla} \times \boldsymbol{E}=-\frac{1}{c} \boldsymbol{\nabla} \times(\boldsymbol{v} \times \boldsymbol{B})=0
$$

Ampere's equation

$$
\boldsymbol{\nabla} \times \boldsymbol{B}=\frac{4 \pi}{c} \boldsymbol{J}
$$

the isothermal equation of state

$$
P=\rho a^{2}
$$


and the no monopoles condition

$$
\boldsymbol{\nabla} \cdot \boldsymbol{B}=0
$$

We have used constant $a^{2}$ to rewrite the pressure gradient as $-\nabla p / \rho=-a^{2} \nabla \ln \rho$. The isothermal approximation is justified in section 2.8. The Coriolis and centrifugal forces appear in Eq. 2.71 as we work in a corotating frame (see section 2.5).

To gain further insight, we rewrite Eq. 2.71 using the vector identity $\boldsymbol{v} \cdot \boldsymbol{\nabla} \boldsymbol{v}=$ $\boldsymbol{\nabla}\left(v^{2} / 2\right)-\boldsymbol{v} \times(\boldsymbol{\nabla} \times \boldsymbol{v})$ to obtain

$$
\nabla W=\boldsymbol{v} \times(2 \boldsymbol{\Omega}+\boldsymbol{\nabla} \times \boldsymbol{v})+\frac{1}{\rho c} \boldsymbol{J} \times \boldsymbol{B}
$$

where

$$
W \equiv \frac{1}{2} v^{2}+a^{2} \ln \rho+U
$$

Constants of the motion can be derived by dotting Eq. 2.77 with $\boldsymbol{B}$ to eliminate the Lorentz force. We find

$$
\boldsymbol{B} \cdot \boldsymbol{\nabla} W=-(2 \boldsymbol{\Omega}+\boldsymbol{\nabla} \times \boldsymbol{v}) \cdot(\boldsymbol{v} \times \boldsymbol{B})=0,
$$

since the electric field vanishes in the co-rotating frame (Spruit 1996). Hence $W$, the Bernoulli constant, is constant along field lines. Another way to understand the work done on the gas is to dot Eq. 2.77 with $\boldsymbol{v}$ :

$$
\rho \boldsymbol{v} \cdot \nabla W=\nabla \cdot(\rho \boldsymbol{v} W)=-\frac{1}{c} \boldsymbol{v} \cdot(\boldsymbol{B} \times \boldsymbol{J})=\boldsymbol{J} \cdot \boldsymbol{E}=0 .
$$


In the rotating frame, work is done on the gas by $-\nabla U$, while in the inertial frame the electromagnetic field performs $\boldsymbol{J} \cdot \boldsymbol{E}$ work on the gas (Spruit 1996).

To understand the magnetic field structure in more detail, we take the cross product of Eq. 2.77 with $\boldsymbol{B}$ to obtain the equation of trans-field force balance. Solving this equation for the component of current perpendicular to $\boldsymbol{B}$ we find

$$
\frac{4 \pi}{c} \boldsymbol{J}_{\perp} \equiv \frac{4 \pi}{c}(\boldsymbol{J}-\boldsymbol{b} \boldsymbol{b} \cdot \boldsymbol{J})=\frac{1}{v_{A}^{2}} \boldsymbol{B} \times[\boldsymbol{\nabla} W+(2 \boldsymbol{\Omega}+\boldsymbol{\nabla} \times \boldsymbol{v}) \times \boldsymbol{v}]
$$

Here $v_{A}=B / \sqrt{4 \pi \rho}$ is the Alfvén speed. This equation describes the perpendicular currents that must flow in order to achieve perpendicular force balance. In axisymmetry, this equation is often called the modified Grad-Shafronov equation (Heinemann \& Olbert 1978; Lovelace et al. 1986). Perpendicular currents arise due to either vorticity in the flow, or variation of the Bernoulli constant from one field line to the next. In the dead zone, the fact that $\boldsymbol{v}=0$, and the further assumption that $W$ is constant at the base, implies that $\boldsymbol{J}_{\perp}=0$ in the dead zone. Parallel currents are determined from $\boldsymbol{J}_{\perp}$ by charge conservation, $\boldsymbol{\nabla} \cdot \boldsymbol{J}=0$.

An order of magnitude estimate for the fields $\delta \boldsymbol{B}$ created by volume currents $\boldsymbol{J}_{\perp}$, compared to the planetary dynamo-generated fields $\boldsymbol{B}_{p}$ is

$$
\frac{B_{\perp}}{B_{p}} \sim \frac{r}{B} \frac{4 \pi}{c} J_{\perp} \sim \frac{\max \left(a^{2}, v^{2}, \Omega r v,(\Omega r)^{2}\right)}{v_{A, p}^{2}}
$$

where $v_{A, p}=B_{p} / \sqrt{4 \pi \rho}$. The terms separated by commas on the right hand side of Eq. 2.82 are estimates of the individual terms in Eq. 2.81. This estimate shows that volume currents can only significantly perturb the field out near the Alfvén radius where $v \sim \Omega r \sim v_{A, p}$. As we now discuss, at much smaller radii, of order the dead zone radius, the field is already strongly perturbed by current sheets. 
Mestel (1968) and Mestel \& Spruit (1987) discussed the matching conditions between the dead and wind zones. The finite velocity in the wind zone acts to decrease the pressure there relative to the dead zone. Integrating the momentum equation across the dead zone-wind zone boundary, the total gas plus magnetic pressure must be continuous, so that the magnetic field strength must increase moving from the dead to the wind zone. This implies the existence of a current sheet separating the dead and wind zone boundaries, as shown in Figure 1.7. Letting the subscripts "d" and "w" denote quantities just inside the dead and wind zones, respectively, total pressure continuity can be written

$$
P_{d}+\frac{B_{d}^{2}}{8 \pi}=P_{w}+\frac{B_{w}^{2}}{8 \pi}
$$

For identical conditions at the base, the Bernoulli equation relates the pressures as $P_{w} \simeq P_{d} \exp \left(-v^{2} / 2 a^{2}\right)$. Considering only the dipole field from the planet, $B_{p}$, and the field $\delta B \simeq 2 \pi K / c$ produced by current per unit length, $K$, the fields in the dead and wind zones are $B_{w}=B_{p}+\delta B$ and $B_{d}=B_{p}-\delta B$. Plugging in to Eq. 2.83 the solution for the line density is

$$
\frac{K}{c}=\frac{P_{d}}{B_{p}}\left(1-e^{-v^{2} / 2 a^{2}}\right)
$$

The ratio of the field produced by the sheet current compared to that from the planet's core is then

$$
\frac{\delta B}{B_{p}} \simeq \frac{1}{4} \beta_{d}\left(1-e^{-v^{2} / 2 a^{2}}\right)
$$

where $\beta_{d}=8 \pi P_{d} / B_{p}^{2}$ is the beta for the planetary field just inside the dead zone. 
Inside the sonic point in the wind zone, $v \ll a$ and sheet currents only slightly perturb the field since $\delta B / B \sim \beta_{d} v^{2} / 8 a^{2} \sim\left(v / 2 v_{A}\right)^{2} \ll 1$. Outside the sonic point, where $v / a \gg 1$, we find $\delta B / B_{p} \sim \beta_{d} / 4$, which increases outward. Hence the field configuration is expected to be significantly altered from the dipole outside the $\beta_{d} \sim 1$ point in the wind zone. As we have assumed that $\beta \gg 1$ at the sonic point, we expect the field to be altered in between the sonic and Alfvén points.

In addition to the sheet currents at the dead zone-wind zone boundary, there is a sheet current at the equator in the wind zone. This sheet current causes the reversal in sign of the field near the equator, approaching the split monopole form $\boldsymbol{B} \propto r^{-2} \boldsymbol{e}_{r}$ sufficiently distant from other current sources near the planet. Since $K \propto B_{r} \propto 1 / r^{2}$ in the wind zone, and $K \propto \beta_{d}$ increases in the dead zone, we expect the maximum current to occur near the cusp in the magnetic field. The polar dead zone is expected to have a smooth transition from dead to wind zone, as Figure 2.7 shows a gradual transition. We expect volume currents in this transition, rather than true sheet currents.

Based on these analytic estimates, an approximate field geometry in the wind zone is roughly dipolar inside the dead zone radius and roughly straight field lines outside. To go beyond this would require a detailed solution of the trans-field force balance for the field geometry, which is beyond the scope of the present work.

\subsection{Appendix: Mean Free Paths, Ion-Neutral Drift \& Ohm's law}

In this section we discuss the relative motion of the e-p-H gas as well as the magnetic field for the conditions relevant to hot Jupiters (see Figure 2.9). Equations and 
collision rates are taken from Schunk \& Nagy (2004), SN hereafter.

To simplify the calculation, we assume all three species are isothermal with temperature $T$, and we work in the "diffusion approximation" in which inertial terms are ignored in the fluid equations of each species. Let $\boldsymbol{v}_{j}$ be the mean velocity of species $j, \boldsymbol{E}$ the electric field, and $\nu_{j k}$ the momentum-transfer collision rate between species $j$ and $k$. Momentum conservation implies $n_{j} m_{j} \nu_{j k}=n_{k} m_{k} \nu_{k j}$. We follow Braginskii (1965) and ignore anisotropy in the collision frequencies, using the parallel value here for simplicity. The effective gravity be denoted $\boldsymbol{g}=-\boldsymbol{\nabla} U$ and the pressures are $P_{j}=n_{j} k_{b} T$. The momentum equations for e, $\mathrm{p}$ and $\mathrm{H}$ are, respectively,

$$
\begin{array}{r}
-e n_{e}\left(\boldsymbol{E}+\frac{1}{c} \boldsymbol{v}_{e} \times \boldsymbol{B}\right)-\boldsymbol{\nabla} P_{e} \\
+n_{e} m_{e}\left[\boldsymbol{g}+\nu_{e p}\left(\boldsymbol{v}_{p}-\boldsymbol{v}_{e}\right)+\nu_{e H}\left(\boldsymbol{v}_{H}-\boldsymbol{v}_{e}\right)\right]=0 \\
e n_{p}\left(\boldsymbol{E}+\frac{1}{c} \boldsymbol{v}_{p} \times \boldsymbol{B}\right)-\boldsymbol{\nabla} P_{p} \\
+n_{p} m_{p}\left[\boldsymbol{g}+\nu_{p e}\left(\boldsymbol{v}_{e}-\boldsymbol{v}_{p}\right)+\nu_{p H}\left(\boldsymbol{v}_{H}-\boldsymbol{v}_{p}\right)\right]=0 \\
-\nabla P_{H}+n_{H} m_{p}\left[\boldsymbol{g}+\nu_{H e}\left(\boldsymbol{v}_{e}-\boldsymbol{v}_{H}\right)+\nu_{H p}\left(\boldsymbol{v}_{p}-\boldsymbol{v}_{H}\right)\right]=0 .
\end{array}
$$

In order, the terms in Eq. 2.86 are the Lorentz force, the pressure gradient, gravitational force, and collision drag force between e-p and e-H. We further impose charge neutrality

$$
n_{e}=n_{p}
$$

and define the center of mass velocity (used throughout the paper)

$$
\boldsymbol{v}=\frac{n_{e} m_{e} \boldsymbol{v}_{e}+n_{p} m_{p} \boldsymbol{v}_{p}+n_{H} m_{p} \boldsymbol{v}_{H}}{m_{e} n_{e}+m_{p} n_{p}+m_{p} n_{H}} \simeq \frac{n_{p} \boldsymbol{v}_{p}+n_{H} \boldsymbol{v}_{H}}{n_{p}+n_{H}}
$$




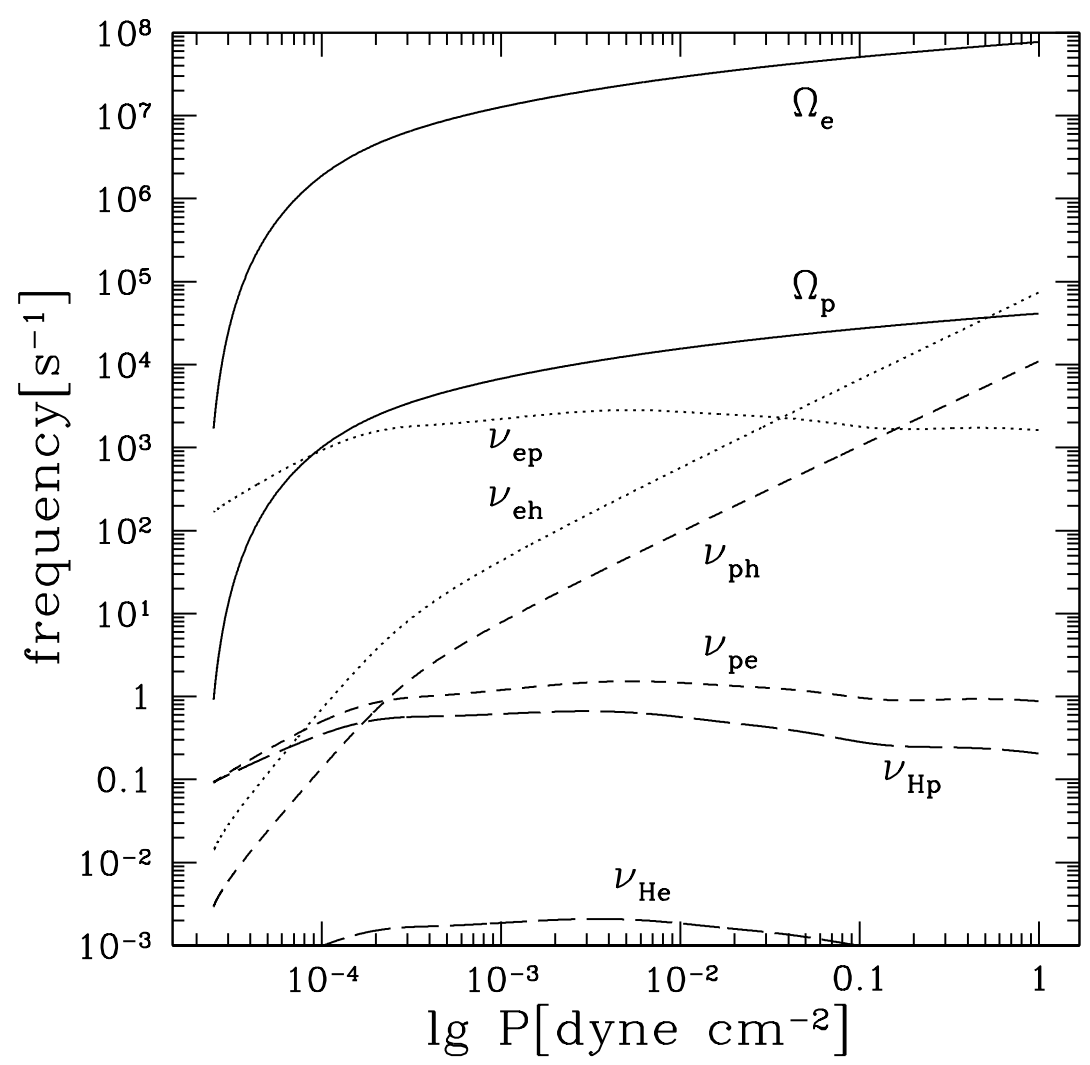

Fig. 2.16. - Gyration and collision frequencies versus depth for the model shown in Figure 2.9.

where the second equality is valid in the $m_{e} / m_{p} \ll 1$ limit.

The momentum transfer and cyclotron frequencies are given in Table 2.2. They are shown as a function of depth in Figure 2.16 using values of $n_{p}, n_{H}, T$ and $B=$ $B_{\mathrm{J}, \text { eq }}(R / r)^{3}$ for the hydrostatic model of the equatorial dead zone shown in Figure 2.9. For these parameters, the gyration frequencies are larger than the e and $\mathrm{p}$ collision frequencies over the entire $\mathrm{H}^{+}$and $\mathrm{H}$ layers, implying motion of both $\mathrm{e}$ and p perpendicular to magnetic field lines is greatly restricted by the magnetic field. Collisions with e are dominated by p well into the $\mathrm{H}$ layer, while $\mathrm{H}$ dominates collisions 


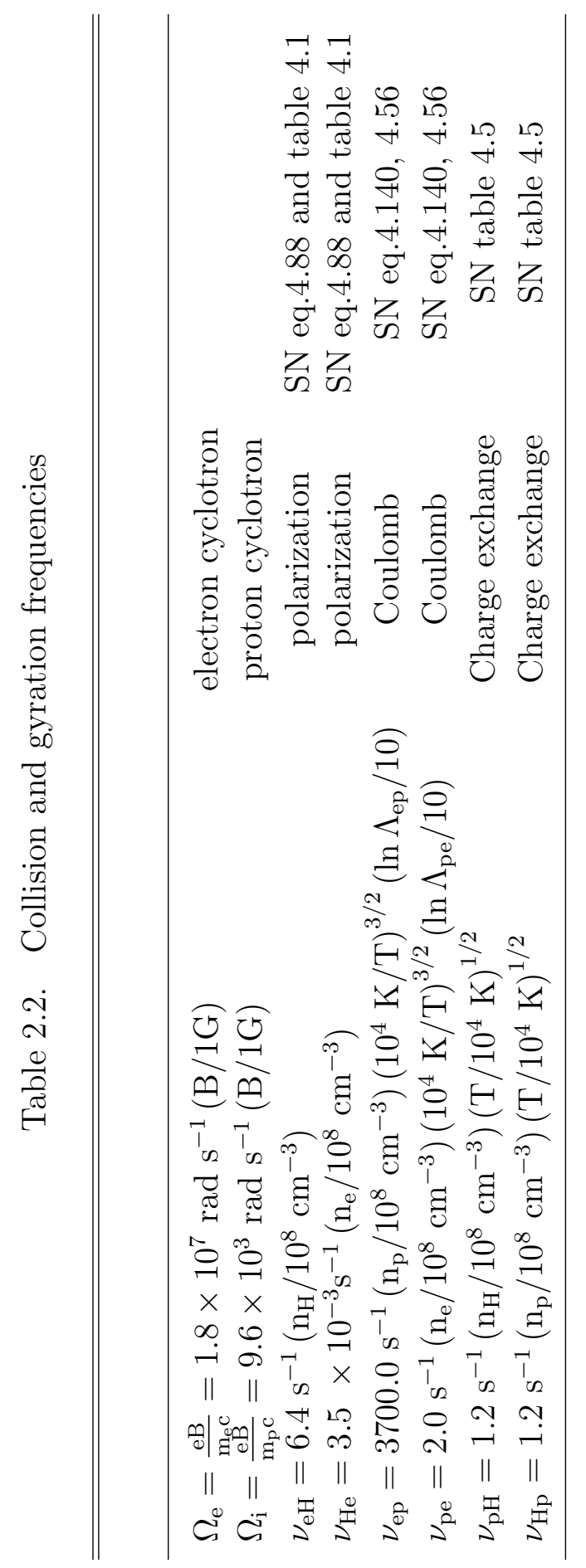


with $\mathrm{p}$ deeper than the $\mathrm{H}-\mathrm{H}^{+}$transition. $\mathrm{H}$ atom collisions with $\mathrm{p}$ dominate over those from e.

For a hydrogen atom traveling at a typical speed $c_{H} \simeq 10 \mathrm{~km} \mathrm{~s}^{-1}\left(T / 10^{4} \mathrm{~K}\right)^{1 / 2}$, the mean free path against collisions with $\mathrm{p}$ is $\simeq c_{H} / \nu_{H p} \simeq 10 \mathrm{~km}\left(10^{8} \mathrm{~cm}^{-3} / n_{p}\right)$. The proton density is sufficiently large that the mean free path is smaller than the scale height, $\simeq r^{2} / \lambda R$, over the entire range shown in Figure 2.9. We conclude that, due to proximity to the star, the high temperature and large scale height cause the density to be large enough that a fluid treatment is appropriate. The hot Jupiter magnetospheres discussed here are collisional, and the exobase is sufficiently distant from the planet to be of little practical importance. A corollary is that $\mathrm{H}$ atoms do not fly ballistically through the magnetosphere, and hence acceleration by stellar tidal gravity or radiation pressure does not cause acceleration of $\mathrm{H}$ atoms away from the planet (Lyman $\alpha$ radiation pressure is only effective in a thin outer skin where Lyman $\alpha$ optical depth is less than unity (Murray-Clay et al. 2009)). Rather, acceleration induces a drift velocity, which we now estimate.

Ignoring the $\nu_{H e}$ term in Eq. 2.88, the ion-neutral drift velocity is

$$
\boldsymbol{v}_{H}-\boldsymbol{v}_{p} \simeq \frac{1}{\nu_{H p}}\left(\boldsymbol{g}-\frac{1}{n_{H} m_{p}} \nabla P_{H}\right)
$$

For a simple estimate of the drift speed, we ignore the pressure gradient term, and use fiducial values $g \simeq 10^{3} \mathrm{~cm} \mathrm{~s}^{-2}$ and $\nu_{H p} \simeq 1 \mathrm{~s}^{-1}$, giving $v_{H}-v_{p} \sim 10 \mathrm{~m} \mathrm{~s}^{-1}$, and a drift time over a distance $R_{J}$ of months. However, ignoring the pressure gradient is a poor approximation. In the $\mathrm{H}$ layer, hydrogen atoms provide the pressure support and so hydrostatic balance implies the quantity in parenthesis in Eq. 2.91 is small. In the $\mathrm{H}^{+}$layer, in photoionization equilibrium, the same cancellation occurs, but for a different reason. There, both protons and electrons provide the pressure support, 
and so the proton scale height is $\simeq 2 k_{b} T / m_{p} g$. But in photoionization equilibrium, Eq. 2.41 implies $n_{H} \propto n_{p}^{2}$, giving hydrogen scale height $\simeq k_{b} T / m_{p} g$, so that the terms in parenthesis in Eq. 2.91 very nearly cancel. The deviations from photoionization equilibrium implies the drift velocity is proportional to a factor $n_{p} / n_{\mathrm{eq}}$ in the $\mathrm{H}^{+}$ layer and $n_{\mathrm{eq}} / n_{H}$ in the $\mathrm{H}$ layer, and the drift velocity is much smaller than the naive estimate $\simeq g / \nu_{\mathrm{Hp}}$, except near the $\mathrm{H}-\mathrm{H}^{+}$transition. Hence the drift time over a distance $\simeq R$ is much longer than the photoionization time of $\simeq$ hrs, hence photoionization equilibrium is a good approximation, as little diffusion can occur in between photoionization events.

Next we discuss deviations from perfect flux freezing. To derive Ohm's law, we follow Braginskii (1965) and solve Eq. 2.88 for $v_{H}$, plug the result into Eq. 2.86, and change references frames from $\boldsymbol{v}_{e}$ to $\boldsymbol{v}$ in the Lorentz force, with the result

$$
\begin{array}{r}
\boldsymbol{E}+\frac{1}{c} \boldsymbol{v} \times \boldsymbol{B}=\left(\frac{\boldsymbol{J} \times \boldsymbol{B}}{n_{e} e c}\right)\left[\frac{\rho_{p}}{\rho}-\frac{\rho_{H}}{\rho} \frac{\nu_{H e}}{\nu_{H}}\right]+\frac{\rho_{H}}{\rho} \frac{1}{\nu_{H} c}\left(\boldsymbol{g}-\frac{1}{\rho_{H}} \boldsymbol{\nabla} P_{H}\right) \times \boldsymbol{B} \\
+\frac{\boldsymbol{J}}{\sigma}+\frac{m_{e}}{e} \boldsymbol{g}\left(1+\frac{\nu_{e H}}{\nu_{H}}\right)-\frac{\boldsymbol{\nabla} P_{e}}{e n_{e}}-\frac{\nu_{e H}}{\nu_{H}} \frac{\boldsymbol{\nabla} P_{H}}{e n_{e}} .
\end{array}
$$

Here $\nu_{H}=\nu_{H e}+\nu_{H p}, \sigma^{-1}=\left(m_{e} / n_{e} e^{2}\right)\left(\nu_{e p}+\nu_{e H} \nu_{H p} / \nu_{H}\right)$ is the conductivity, $\rho_{p}=$ $m_{p} n_{p}, \rho_{H}=m_{p} n_{H}$, and $\rho \simeq \rho_{p}+\rho_{H}$. The second term on the left hand side is due to induction. The terms on the right hand side are the Hall term, drift due to net force on the neutrals, the Ohmic term, the (small) term due to gravity on the electrons, the electron pressure gradient term, which gives rise to the charge separation field, and its correction due to collisions with neutrals. The second term on the right hand side may be put in the form of "ambipolar diffusion", in astrophysical parlance, by using the total momentum equation

$$
\rho_{H} \boldsymbol{g}-\nabla P_{H} \simeq-\rho_{p} \boldsymbol{g}+\nabla\left(P_{e}+P_{p}\right)-\frac{1}{c} \boldsymbol{J} \times \boldsymbol{B}
$$


yielding a term

$$
\frac{\boldsymbol{B} \times(\boldsymbol{J} \times \boldsymbol{B})}{\rho c^{2} \nu_{H}}
$$

on the right hand side.

Applying Eq. 2.92 to compute magnetic field evolution requires knowledge currents and particle densities. As argued in Appendix 2.15, the cross-field currents are zero in the dead zone if the Bernoulli constant is uniform at the base of the atmosphere. While true for the simple case considered in this paper (isothermal, hydrostatic equilibrium), non-isothermal conditions and/or fluid motion at the base may induce perpendicular currents.

We now discuss the relative size of terms in Ohm's law. In the $\mathrm{H}^{+}$layer, the Ohmic diffusivity is $\eta=c^{2} / 4 \pi \sigma \simeq\left(c^{2} / 4 \pi\right)\left(m_{e} \nu_{e p} / n_{e} e^{2}\right) \simeq 10^{7} \mathrm{~cm}^{2} \mathrm{~s}^{-1}\left(T / 10^{4} \mathrm{~K}\right)^{3 / 2}$, independent of density. Assuming Ohmic decay is balanced through the induction term, and that the currents are of order $J \sim(c / 4 \pi)(B / r)$, the required (center of mass) fluid velocity is $v \sim \eta / r \sim 10^{-3} \mathrm{~cm} \mathrm{~s}^{-1}$, many orders of magnitude smaller than any characteristic velocity in the problem. Deep in the H layer, Figure 2.16 shows that the collision rates, and hence diffusivity, may increase by an order of magnitude. For nonzero cross field currents, the ratio of the Hall to the Ohmic term is roughly $\sim \Omega_{e} / \nu_{e} \sim 10^{4}$, where $\nu_{e} \equiv \nu_{e p}+\nu_{e H} \nu_{H p} / \nu_{H}$. When significant cross field currents exist, the Hall drift speed can be much larger than the Ohmic drift speed, but still much smaller than the gas sound speed. Lastly, if the neutral drift speed has a cross field component, the second term on the right hand side may generate a fluid velocity $v \sim\left(\rho_{H} / \rho\right)\left(v_{H}-v_{p}\right)$. This drift speed is much larger than the Ohmic drift speed, although it is still much smaller than the sound speed.

We conclude that, for the ionization models discussed in this paper, the ion-neutral 
drift velocity and deviations from flux freezing in the $\mathrm{H}$ and $\mathrm{H}^{+}$layers are small, and single-fluid MHD is a good approximation. 


\section{Chapter 3}

\section{Numerical Simulations with ZEUS-MP}

\subsection{Overview}

Moving beyond the semi-analytic models presented in Chapter 2 requires the use of numerical simulations, both to (1) provide an independent test of the validity of the calculations presented in Trammell et al. (2011), and (2) to relax the assumptions of the analytic model. This motivates the use of a well-tested code for magnetized fluids flow, which is described in the following sections. I postpone the discussion of the specific configuration and boundary conditions for the problem to be solved until Chapter 4, where I describe the adaptation specifically for the simulations of HJ upper atmospheres.

The publicly available magnetohydrodynamics (MHD) code ZEUS-MP (see Hayes et al. 2006, and references therein) solves the ideal, compressible equations that describe the physical state of a fluid element through the mass density $(\rho)$, velocity $(\boldsymbol{v})$, internal energy density $(e)$, and magnetic field $(\boldsymbol{B})$. The ZEUS family of codes 
has seen widespread use in solving a variety of astrophysical problems, including the formation of galactic spiral arms (e.g., Martos et al. 2004a,b), turbulence in molecular clouds relevant for the early stages of star formation (e.g., Mac Low 1999), and even studies of planetary nebulae (e.g., García-Segura et al. 1999), to name just a few.

The ZEUS-MP code described here differs from previous versions of the code such as the highly-documented ZEUS-2D (Stone \& Norman 1992a,b) and ZEUS-3D, which lacks any peer-reviewed literature documentation, and the partially documented "version 1" of ZEUS-MP that has been publicly available since 1999. The compelling goal of ZEUS-MP is to, for the first time, unifying 3D hydrodynamics (HD) and 3D MHD capability in a software platform that is adapted for execution on massively parallel (hence the 'MP' title) computer architectures. Since the original release of the ZEUS codes, the computing architecture landscape has shifted dramatically toward largescale multicore machines with large reservoirs of fast memory. Thus, the algorithms of ZEUS-MP are designed with the scalability that accompanies parallel execution in mind. The actual equations that the ZEUS-MP code solves for fluids are now described in the following section. 


\subsection{Relevant Equations}

The full set of equations (see Eq. (1)-(6) in Hayes et al. 2006) that the code solves in the case without radiation, or explicit heating/cooling prescriptions reduce to

$$
\begin{aligned}
\frac{D \rho}{D t} & =-\rho \nabla \cdot \boldsymbol{v} \\
\rho \frac{D \boldsymbol{v}}{D t} & =-\nabla P+\frac{1}{4 \pi}(\nabla \times \boldsymbol{B}) \times \boldsymbol{B}-\rho \nabla \Phi \\
\rho \frac{D}{D t}\left(\frac{e}{\rho}\right) & =-p \nabla \cdot \boldsymbol{v} \\
\frac{\partial \boldsymbol{B}}{\partial t} & =\nabla \times(\boldsymbol{v} \times \boldsymbol{B}),
\end{aligned}
$$

where the comoving (Lagrangian) derivative is defined as

$$
\frac{D}{D t} \equiv \frac{\partial}{\partial t}+\boldsymbol{v} \cdot \nabla
$$

Equations 3.1-3.4 are the mass continuity equation, momentum equation, energy equation, and induction equation, respectively. Here, $e$ is the internal energy and $\Phi$ is the gravitational potential. For the purposes of application to the remainder of the discussion of ZEUS-MP in the context of this thesis, the simulations are axisymmetric (e.g., all $\partial / \partial \phi=0$ ), and make use of spherical polar coordinates $(r, \theta, \phi)$. This coordinate system naturally has a spherical surface as the inner radial boundary to the computational domain (so the physical boundary itself lies along one of the coordinate directions, and it simplifies the data post-processing calculations because it eliminates the need for coordinate transformations and a recast of all vector field quantities in a different orthonormal basis.

The terms on the right hand side of Equation 3.2 are the force per unit volume due to thermal pressure gradients used to compute accelerations of fluid elements, 
Lorentz $(\boldsymbol{J} \times \boldsymbol{B})$ forces, and gradients of the gravitational potential, respectively. For completeness, explicit heating and cooling terms would need to be included as well in order to more realistically compute the temperature structure throughout the ionized layers. However, following the model described in detail in Chapter 2, I employ an isothermal equation of state, which closes Equations 3.1-3.4 above) but without any explicit heating/cooling terms.

The time-evolution of the magnetic field in Equation 3.4 assumes ideal MHD (i.e., zero drift velocity of ions relative to electrons and neutrals in the fluid. Instead of an explicit prescription for the resistivity, a value of zero resistivity is assumed for evolving $\boldsymbol{B}$, which is reasonable for many astrophysical plasmas where the ionization fraction is fairly high. Clearly, there will be situations in which the ideal-MHD assumption becomes inaccurate (e.g., regions of high density and weak magnetic field, or very low density and strong magnetic field), and Stone (1999) has added additional modules to the serial (outdated) ZEUS code to compute updates to $\boldsymbol{B}$ that account for non-ideal MHD effects. For my purposes here, I use a specific version of the ZEUS family, ZEUS-MP, whose numerical method is now described in more detail in the following section.

\subsection{Numerical Methods}

The ZEUS-MP code is a highly-parallelized code that is optimized for speed, taking advantage of the Message-Passing Interface (MPI) Fortran libraries. The code also employs artificial numerical viscosity for more accurate shock capture at contact discontinuities (e.g., a large jump in fluid density and/or internal energy density between adjacent cells). This latter feature is similar to previous serial (i.e., single process) incarnations of the predecessor ZEUS codes that have been tested extensively to as- 


\section{ZEUS-MP}

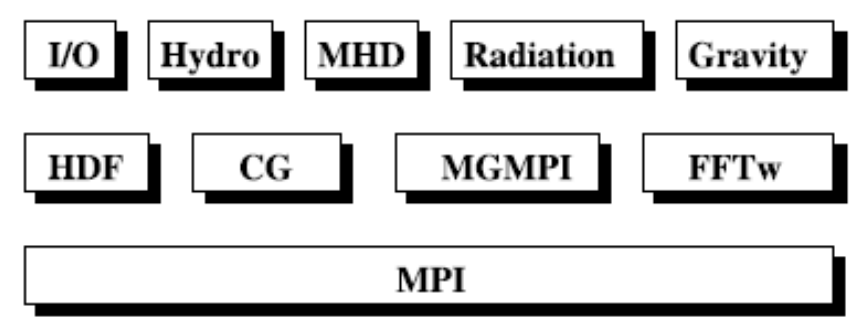

Fig. 3.1. - Primary software module implementation for the ZEUS-MP code (Hayes et al. 2006, Fig. 1).

certain their accuracy and limitations (Hayes et al. 2006; Stone \& Norman 1992a,b), the discussion of which I postpone until Section 3.6. What follows here is an overview of features unique to ZEUS-MP.

The many specific modules that make up the architecture of the ZEUS-MP code perform various calculations as well as input and output (I/O) subroutines. Figure 3.1 schematically illustrates the implementation of various components (some are not used in this application to planetary atmospheres).

For MHD, the solution to the fluid equations is split into the following solution steps: (1) the source step, in which the code solves the equations

$$
\begin{aligned}
\rho \frac{\partial \boldsymbol{v}}{\partial t} & =-\nabla p-\nabla\left(B^{2} / 8 \pi\right)-\rho \nabla \Phi \\
\frac{\partial e}{\partial t} & =-p \nabla \cdot \boldsymbol{v},
\end{aligned}
$$

(2) the "MOCCT" step that combines the constrained transport algorithm for updating the components of $\varepsilon=(\boldsymbol{v} \times \mathbf{B})$ from Evans \& Hawley (1988) with the method 
of characteristics (MOC) treatment for Alfvén waves:

$$
\begin{aligned}
\left.\rho \frac{\partial \boldsymbol{v}}{\partial t}\right|_{\text {final }} & =\left.\rho \frac{\partial \boldsymbol{v}}{\partial t}\right|_{\text {source step }}+\frac{1}{4 \pi}(\mathbf{B} \cdot \nabla) \mathbf{B} \\
\frac{d}{d t} \int_{S} \mathbf{B} \cdot d \mathbf{S} & =\varepsilon \cdot d \mathbf{l} . \\
\varepsilon & =\left(\boldsymbol{v}-\boldsymbol{v}_{\mathrm{g}}\right) \times \mathbf{B},
\end{aligned}
$$

and finally (3) a transport step in which mass density, internal energy density $e$, and magnetic field quantities are updated for each fluid element and evolved according to finite-difference expressions analogous to Equations 3.1-3.4 above:

$$
\begin{aligned}
\frac{d}{d t} \int_{V} \rho d V & =-\oint_{d V} \rho\left(\boldsymbol{v}-\boldsymbol{v}_{\mathrm{g}}\right) \cdot \mathrm{d} \mathbf{S} \\
\frac{d}{d t} \int_{V} \rho \mathbf{v} d V & =-\oint_{d V} \rho \boldsymbol{v}\left(\boldsymbol{v}-\boldsymbol{v}_{\mathrm{g}}\right) \cdot \mathrm{d} \mathbf{S} \\
\frac{e}{d t} \int_{V} e d V & =-\oint_{d V} e\left(\boldsymbol{v}-\boldsymbol{v}_{\mathrm{g}}\right) \cdot \mathrm{d} \mathbf{S},
\end{aligned}
$$

where $\boldsymbol{v}_{\mathrm{g}}$ is the local grid velocity. The ZEUS-MP code treats two families of MHD waves: (1) longitudinal (fast/slow magnetosonic), and (2) Alfvén waves. For numerical stability the algorithm design from (Hawley \& Stone 1995), implements updates to the fluid velocity components due to momentum transport just before the fluid advection step.

For stability and accuracy in evolving the magnetic field components as a function of time, ZEUS-MP employs the Modified Method of Characteristics (MMOC) numerical scheme of Hawley \& Stone (1995). This method uses a staggered (i.e., offset from cell centers) grid of electromotive force (EMF) values associated with each fluid element and the evolution substep that I mentioned in the previous paragraph in between source and transport steps, which guarantees that any non-zero magnetic 
divergence $\nabla \cdot \boldsymbol{B}$ will not grow in time. So this means that as magnetic field components are evolved in time, the corresponding components of the EMF $\varepsilon_{1}, \varepsilon_{2}, \varepsilon_{3}$ are updated using the magnetic field components $b_{1}, b_{2}$, and $b_{3}$ that are used to compute the Alfvén characteristics. Numerically, a module then computes $\nabla \times \varepsilon$ to update the field components $b_{1}, b_{2}, b_{3}$.

Having described the details of the numerical method, the next section describes the principle components of control for the simulation time step.

\subsection{Time Step Control}

For any numerical code, stability and accuracy of the calculations requires precise control of the simulation time step. The fluid Courant factor $C_{\mathrm{fl}}$ specifies the fraction of the minimum timescale that information can propagate through the length scale of a cell (i.e., $\Delta t=\left(C_{\mathrm{fl}} \times\right.$ length $) /$ time $)$. The numerical scheme requires this minimum timescale to computing the new time step for all fluid elements. In ZEUS-MP, a separate time step is evaluated for each characteristic velocity and then combined into the general expression that regulates the time step:

$$
\Delta t_{\text {new }}=\frac{C_{\mathrm{fl}}}{\sqrt{\Delta t_{\mathrm{cs}}^{-2}+\Delta t_{v 1}^{-2}+\Delta t_{v 2}^{-2}+\Delta t_{v 3}^{-2}+\Delta t_{\mathrm{al}}^{-2}+\Delta t_{a v}^{-2}}} .
$$


The $\Delta t^{-2}$ terms are squares of minimum values of

$$
\begin{aligned}
\Delta t_{\mathrm{cs}}^{-2} & =\gamma(\gamma-1)\left(\frac{e}{\rho}\right)\left(\frac{1}{\Delta x_{\mathrm{min}}^{2}}\right) \\
\Delta t_{v 1}^{-2} & =\left(\frac{v 1-v g 1}{d x 1 a}\right)^{2} \\
\Delta t_{v 2}^{-2} & =\left(\frac{v 2-v g 2}{g 2 b \times d x 1 a}\right)^{2} \\
\Delta t_{v 3}^{-2} & =\left(\frac{v 3-v g 3}{g 31 b \times g 32 b \times d x 3 a}\right)^{2} \\
\Delta t_{\mathrm{al}}^{-2} & =\frac{\overline{b 1}^{2}+\overline{b 2}^{2}+\overline{b 3}^{2}}{4 \rho(\Delta x)^{2}} \\
\Delta t_{\mathrm{av}}^{-2} & =\left(4 q\left|\frac{d v}{d x}\right|_{\max }\right)^{-2} .
\end{aligned}
$$

In the expressions above, vgi are the components of the grid velocity (usually set to zero), dxia terms are the lengths of a cell along the $x i$-direction, and the metric terms $g 2 b, g 31 b$, and $g 32 b$ depend on the coordinate system used. The $\Delta t$ values in the equations above represent the local gas sound crossing time, local fluid crossing time across each cell along each of three possible coordinate directions, the local Alfvén wave crossing time, and the viscous timescale.

After the transport step is complete, the main program calls a subroutine that uses all of the most recent fluid quantities to evaluate Equations 3.15-3.20, and compute a new time step for the following source step using Equation 3.14. The user has control over the limiting behavior of the time step through optional manual override of $\Delta t_{\text {min }}$ as a fraction of the previous result from evaluating Equation 3.14, as well as through a manual setting of the Courant factor $C_{\mathrm{fl}}$ (usually $C_{\mathrm{fl}}=0.5$ ). Armed with the above descriptions of time step control, I now highlight to the general operation of the code in more detail. 


\subsection{Staggered Grid Topology \& Workflow}

Figure 3.2 illustrates the main work flow for the ZEUS-MP code, showing the order of the source and the transport steps in the overall program algorithm. Figures 3.3 and

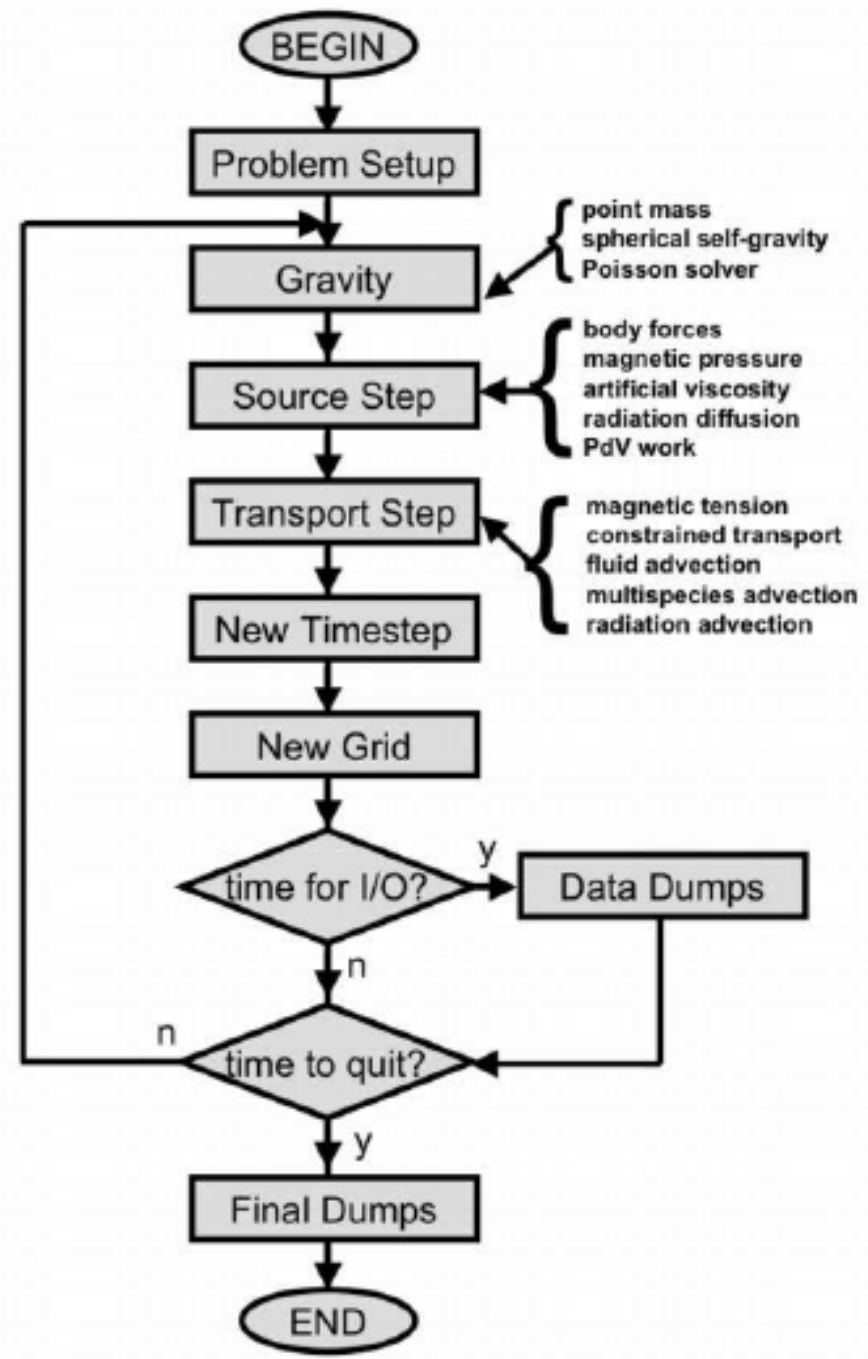

Fig. 3.2.- Workflow/program control for the ZEUS-MP code (taken from Hayes et al. 2006, Fig. 2).

3.4 illustrate the storage locations for the EMFs, as well as the other primary fluid variables: the 1-, 2-, and 3-velocity $\left(v_{1}, v_{2}, v_{3}\right)$, mass density $\rho$, and internal energy density $e$, and their relations to the cell centers. For each coordinate in the basis, the 
MMOC algorithm updates the velocities and evolves the magnetic field using

$$
\begin{aligned}
\frac{\partial v}{\partial t} & =\frac{B_{x}}{\rho} \frac{\partial B}{\partial x}-\frac{\partial}{\partial x}\left(v_{x} v\right) \\
\frac{\partial B}{\partial t} & =B_{x} \frac{\partial v}{\partial x}-\frac{\partial}{\partial x}\left(v_{x} B\right)
\end{aligned}
$$

An example update to the fluid momentum in the 3-direction, including the acceleration from transverse (slow) Aflvén waves is

$$
\rho \frac{\partial v_{3}}{\partial t}=-\nabla_{(3)}\left(B^{2} / 8 \pi\right)+\frac{1}{4 \pi}(\boldsymbol{B} \cdot \nabla) \mathrm{B}_{3}
$$

For full details, including the decomposition of the fluid equations into finite difference equations used by the ZEUS-MP code, see Appendix C of Hayes et al. (2006). Figure

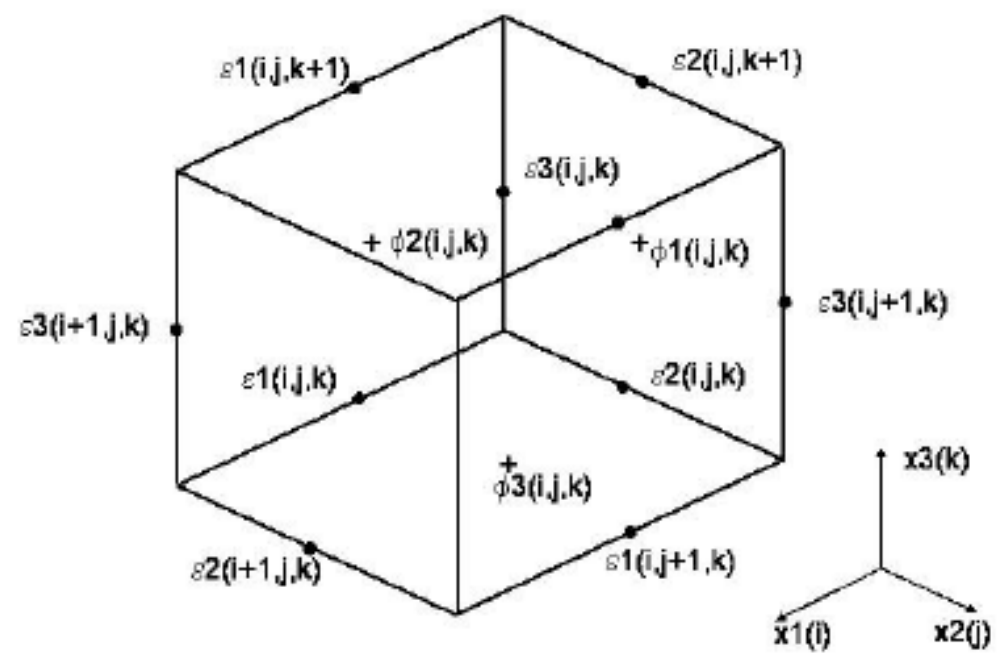

Fig. 3.3.- Illustration of the centering of the EMF variables $\left(\varepsilon_{1}, \varepsilon_{2}, \varepsilon_{3}\right)$ used to update the magnetic field in the ZEUS-MP code (taken from Hayes et al. 2006, Fig. 3). The $i$ component of $\varepsilon_{i}$ is centered on the $(i+1)$ face. For $2 \mathrm{D}$ problems, the cube collapses in the 3 -direction, which places $\varepsilon_{3}$ at the $x 1-x 2$ corners.

3.5 illustrates schematically how the update for the 3-component of the EMF $\varepsilon 3_{i j k}$ requires the solution of $1 \mathrm{D}$ characteristic equations for Alfvén waves confined to the 


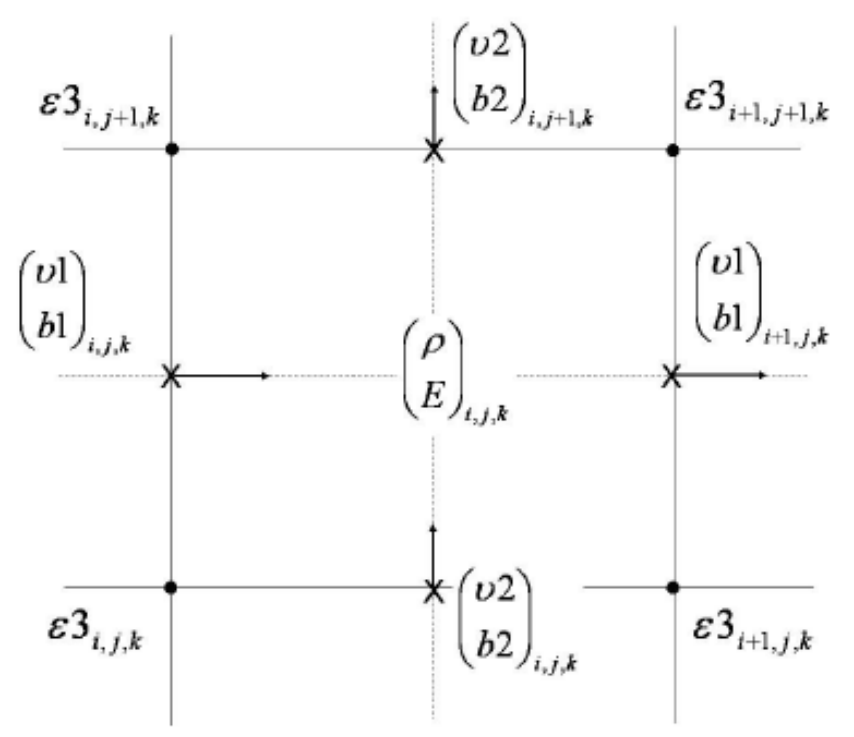

Fig. 3.4.- A 2D-slice $(x 1-x 2)$ through the unit grid cell from Figure 3.3 above that shows the locations for the four remaining emfs $\left(\varepsilon_{3}\right)$ (taken from Hayes et al. 2006, Fig. 4).

$x 1-x 2$ (e.g., $r-\theta$ plane). In this case, Alfvén waves are propagating along the $x 1$-direction give the solution of the $b 2 v 2$ term, while the $b 1 v 1$ term is due to Alfvén waves propagating along the $x 2$-direction.

In the previous sections, I have summarized the primary motivation for selecting ZEUS-MP for the simulations, and I have provided an overview of the main workings of the code itself. However, what remains is to highlight a few key numerical tests using classic MHD problems with analytic solutions (there are few), each of which are intended to demonstrate the accuracy of the ZEUS-MP solver and justify its use for solving the MHD wind problem. I provide these examples and discuss their context in the following section. 


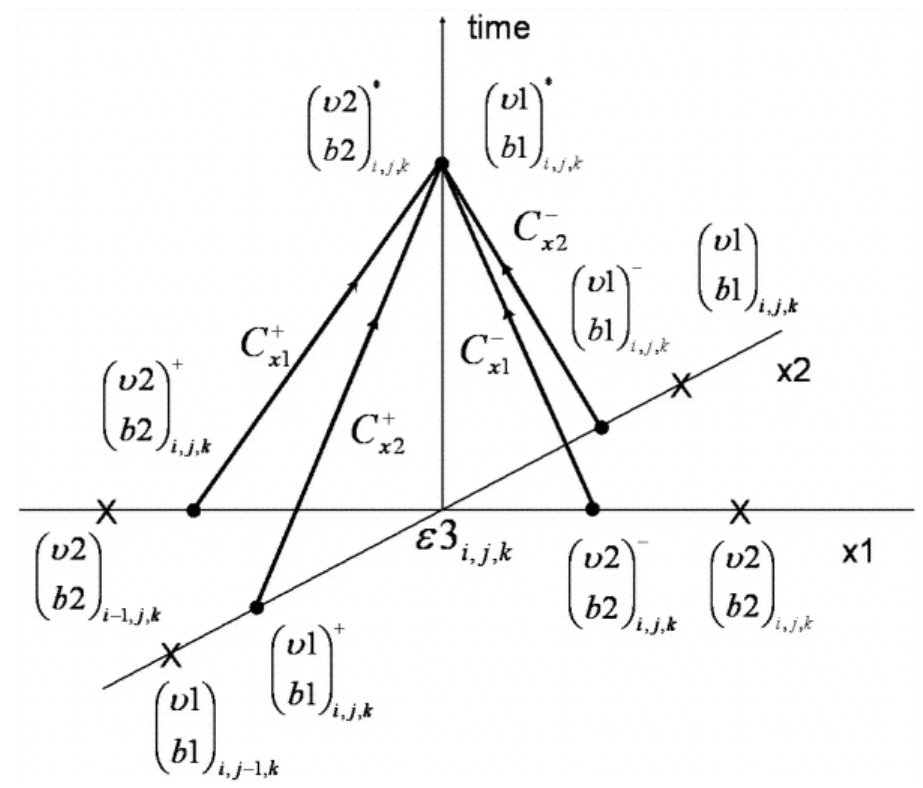

Fig. 3.5.- Schematic illustrating the solution of the 1D Alfvén wave characteristic equations confined to the $x 1-x 2$ plane needed to compute the 3 -component of the emf at the grid location $(i, j, k) \varepsilon 3_{i j k}$. (taken from Hayes et al. 2006, Fig. 5).

\subsection{Example Numerical Tests}

Several numerical tests given in are given in detail in Hayes et al. (2006) have scrutinized the accuracy of the ZEUS-MP solver. One key difference between the MMOC algorithm in ZEUS-MP compared to earlier incarnations of the ZEUS code is that higher resolutions are typically required due to the more diffusive nature of the MMOC numerical scheme. In other words, more fluid elements over the vicinity of a contact discontinuity (i.e., shock) are required. The additional computational load is easily compensated for with the highly-parallelized algorithm native to ZEUS-MP, but the more diffusive nature gives ZEUS-MP a distinct stability advantage for problems involving strong shocks in the presence of strong magnetic fields. Other codes that are even more diffusive are not as appropriate for the MHD wind problem, for in practice they cannot always predict to correct gradients of scalar quantities such as gas density 
and internal energy density.

In the follow three sub-sections, I highlight two numerical tests that Hayes et al. (2006) use to demonstrate the accuracy of various aspects of the ZEUS-MP solver, which are directly relevant to solving the MHD wind problem. The final sub-section demonstrates the convergence of ZEUS-MP with resolution to the semi-analytic hydrodynamic wind solution. Taken together, all three numerical tests for different aspects of the code contribute to its reliability for solving the MHD wind problem, which is the subject of this thesis.

\subsubsection{Sedov-Taylor Blast Wave}

This first test problem is given as an example that specifically tests the limits of the hydrodynamic (HD) algorithms of ZEUS-MP. This classic HD test problem is that of Sedov (1959) that involves a high-energy explosion at the center of a surrounding, cold and homogeneous sphere ignoring the effects of gravity (which is why the problem is sometimes referred to as the "free expansion" problem). The general physical relevance of the problem is its application to understand stellar explosions. For HJ upper atmospheres in particular, these tests are a good demonstration of how the code can handle shocks moving through the computational domain and the stability of boundary conditions at or near the edges of the domain.

One chooses explosion parameters such that the energy density inside of the detonation region is orders of magnitude higher than the surrounding cloud of gas. The resulting expanding shock wave has an analytic self-similar solution with a radius $r_{\mathrm{sh}}$ of the expanding shell of hot material evolving in time according to

$$
r_{\mathrm{sh}}=\xi_{\mathrm{sh}}\left(\frac{E_{0}}{\rho_{0}}\right)^{1 / 5} t^{2 / 5}
$$


and a shock velocity $v_{\text {sh }}$ evolving in time according to

$$
v_{\mathrm{sh}}=\frac{2}{5} \xi_{\mathrm{sh}}\left(\frac{E_{0}}{\rho_{0}}\right)^{1 / 5} t^{-3 / 5} .
$$

The scalars $E_{0}$ and $\rho_{0}$ are the explosion energy and initial density inside the explosion

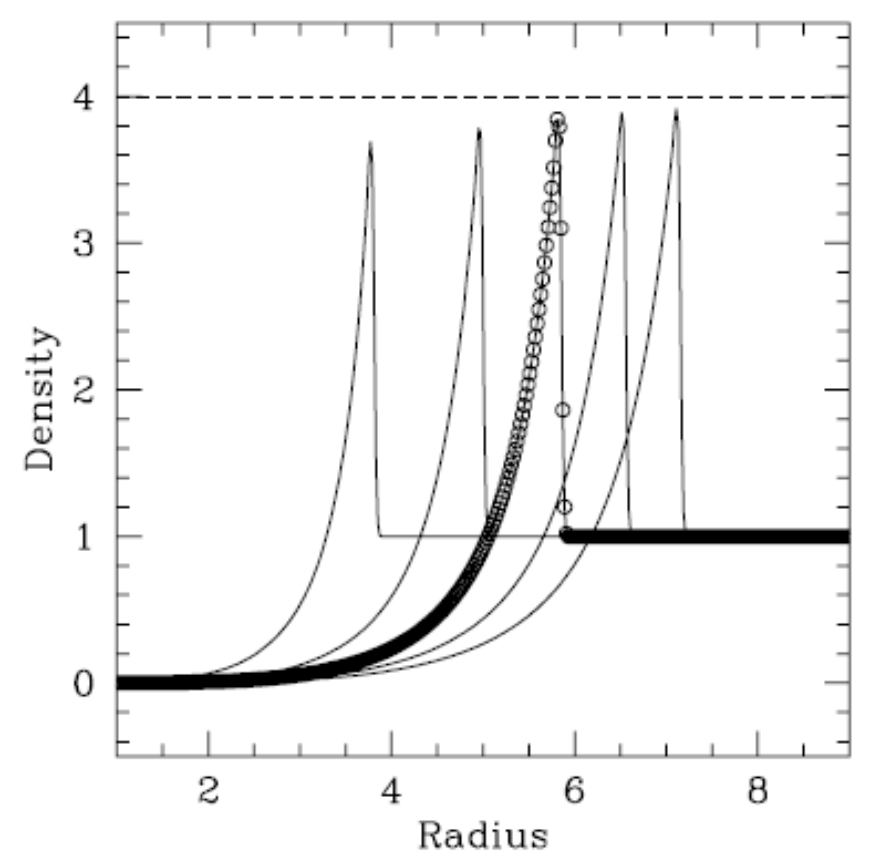

Fig. 3.6.- Density vs. radius for a Sedov-Taylor blast wave, where radius is plotted in units of $\sim 10^{13} \mathrm{~cm}$, along with several analytic calculations for comparison to the open circles that denote grid mesh locations. (taken from Hayes et al. 2006, Fig. 8).

region at time $t=0$, respectively. The coefficient $\xi_{\mathrm{sh}}$ is a dimensionless constant and is 1.15 for an ideal gas with ratio of specific heats $C_{P} / C_{V}=\gamma=5 / 3$. The solution density jump at the shock is $\rho_{s}=4 \rho_{0}$, and Figure 3.6 shows density vs. radius for the Sedov-Taylor blast wave. Density is in units of the peak density. Open circles indicate the densities at mesh locations and solid lines are the analytic solutions, which agree well.

Because ZEUS-MP evolves the gas energy density, rather than the total fluid 
energy, this test in particular demonstrates any non-conservative nature of the ZEUSMP solution scheme. The degree to which the solver can conserve energy across the computational domain is important for the generation of an outflow by thermal pressure gradients.

\subsubsection{Magnetic Braking of an Aligned Rotor}

This test involves the propagation of torsional Alfvén waves that are produced by a rotating gas with a frozen in magnetic field. The initial conditions have a disk of density $\rho_{d}$ and thickness $z_{d}$, rotating at an angular velocity $\Omega_{0}$. The system is embedded in an initially static, ambient medium with density $\rho_{m}$. Both the disk and the medium are threaded by a uniform magnetic field $B$ at time $t=0$. Figure 3.7 shows the solution for the $\phi$-component of the magnetic field which arises purely from Aflvén waves that propagate along the $z$-axis and generate non-zero components for $B_{\phi}$ due to twisting (i.e., winding up) of initially uniform magnetic field lines. The analytic solution comes from Mouschovias \& Paleologou (1980).

This specific test illustrates the accuracy of the induction equation for $B_{\phi}$, which is important because the target simulations involve a hot, rotating object with a strong (initially purely poloidal) magnetic field. This test gives experience with generating $B_{\text {phi }}$ from the twisiting of magnetic field lines. The competition between hydrodynamic and magnetic forces is directly applicable to the MHD wind problem, where pressure gradients and wind ram pressure compete against magnetic tension forces. A direct test of the hydrodynamics solver is presented in the following subsection. 


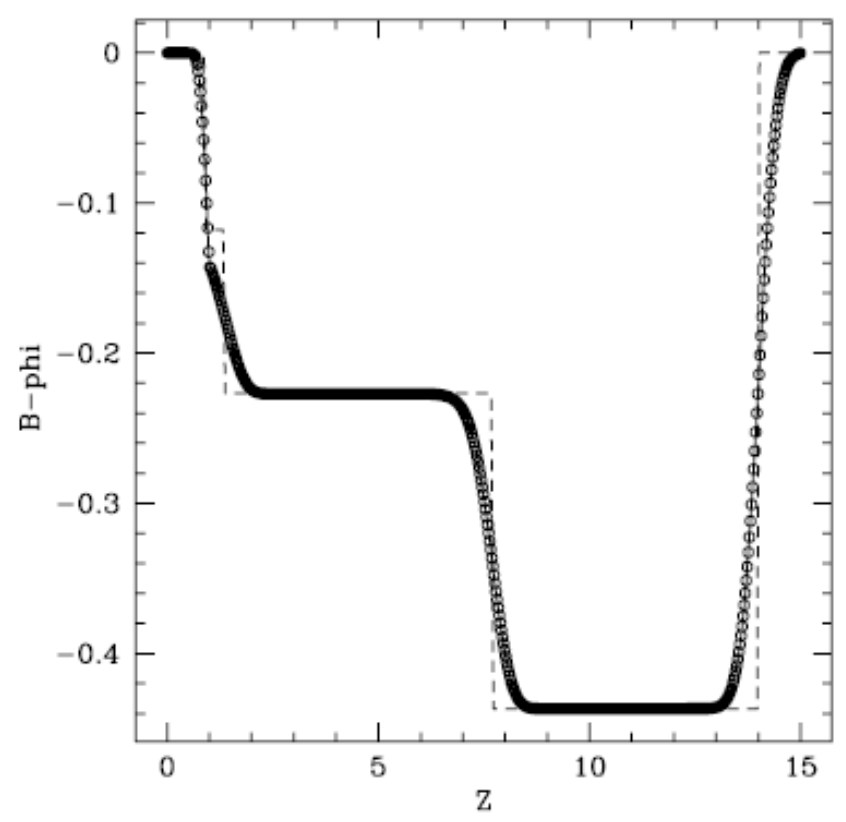

Fig. 3.7. - Similar to Figure 3.6 but now for the MHD aligned rotor test for $B_{\phi}$ vs. $Z$. Here, the dashed lines are the analytic calculations (see Fig. 11 of Hayes et al. 2006, and references therein).

\subsubsection{Spherical Hydrodynamic Wind}

To test the hydrodynamics solver for ZEUS-MP in the context of the outflow problem, a test case for a spherically-symmetric hydrodynamic wind and a physical boundary condition is appropriate. A comparison of the simulation results to test for convergence to a semi-analytic solution demonstrates the accuracy of the hydrodynamics, which is possible with the conditions at the slow critical point (i.e., the sonic point in this case) are specified. The purpose of this section is to outline the semi-analytic solution to the spherical hydrodynamic outflow problem, which involves computing the gas density and velocity as functions of radius $r$, using the conditions at the sonic point as a boundary condition.

The continuity equation (e.g., Equation 3.1 above) should be satisfied throughout 
the computational domain, which can be expressed as

$$
\dot{M}=4 \pi r^{2} \rho v
$$

at radial distance $r$, local gas density $\rho$ and outflow velocity $v$. The other remaining fluid equations that a wind solution must satisfy are the momentum equation and the equation of state, respectively:

$$
\begin{aligned}
v \frac{d v}{d r} & =-\frac{1}{\rho} \frac{d P}{d r}-\frac{G M}{r^{2}} \\
P & =K \rho^{\gamma} .
\end{aligned}
$$

In Equation 3.27, $P$ is the gas pressure, $M$ is the planet mass, and $\gamma$ is the equation of state parameter in Equation 3.28. Using Equations 3.27 and 3.28,

$$
\frac{d P}{\rho}=K \gamma \rho^{\gamma-2} d \rho=d\left(K \frac{\gamma}{\gamma-1} \rho^{\gamma-1}\right) .
$$

For a gas enthalpy $h \equiv K \gamma \rho^{\gamma-1} /(\gamma-1)$, the sound speed $c_{s}=\gamma P / \rho=(\gamma-1) h$. Equation 3.27 can be integrated up to a constant called the Bernoulli constant $B$ :

$$
B=\frac{v^{2}}{2}+\frac{c^{2}}{\gamma-1}-\frac{G M}{r}
$$

Equation 3.30 already provides us with some features of the wind solution. As $r \rightarrow \infty$, the wind speed $v$ asymptotes to a constant value. For a constant $\dot{M}$, Equation 3.26 implies that $\rho \rightarrow 0$ simultaneously. For the polytropic wind solution, Equation 3.30 also implies that the sound speed $c \rightarrow 0$ for $\gamma>1$, which becomes one condition for this parameter in the numerical tests that follow. 
Combining Equations 3.26 and 3.27 yields a simple differential equation for $v$ :

$$
\begin{aligned}
v \frac{d v}{d r} & =-K \gamma \rho^{\gamma-1} \frac{d \ln \rho}{d r}-\frac{G M}{r^{2}} \\
& =-c^{2}\left(-\frac{2}{r}-\frac{d \ln v}{d r}\right)-\frac{G M}{r^{2}} \\
\therefore \frac{d v}{d r} & =\frac{\frac{2 c^{2}}{r}-\frac{G M}{r^{2}}}{v-\frac{c^{2}}{v}} .
\end{aligned}
$$

For a transonic solution, the denominator in Equation 3.33 vanishes when $v=c$, which means that the numerator must simultaneously vanish for the velocity gradient $d v / d r$ to be finite. This location is the slow critical (sonic) point $r_{\mathrm{s}}=G M / 2 c_{\mathrm{s}}^{2}$. Evaluating Equation 3.30 at the sonic point gives

$$
\begin{aligned}
B & =\frac{c_{\mathrm{s}}^{2}}{2}+\frac{c_{\mathrm{s}}^{2}}{\gamma-1}-2 c_{\mathrm{s}}^{2} \\
\therefore B & =\frac{c_{\mathrm{s}}^{2}}{2}\left(\frac{5-3 \gamma}{\gamma-1}\right),
\end{aligned}
$$

which now restricts $\gamma$ further to $1<\gamma<5 / 3$ for gas that is unbound at $r=\infty$ (i.e., $B>0$ ). With $B$ solved for in Equation 3.35, we can substitute into Equation 3.30. The resulting equation can be solved numerically using the dimensionless variables $x \equiv r / r_{\mathrm{s}}$ and $y \equiv v / c_{\mathrm{s}}$ that connect $r$ and $v$ to their values at the sonic point and the value of $B$ from Equation 3.35 above:

$$
\frac{y^{2}}{2}=\frac{1}{\gamma-1} \frac{1}{\left(x^{2} y\right)^{\gamma-1}}-\frac{2}{x}=\frac{c_{\mathrm{s}}^{2}}{2}\left(\frac{5-3 \gamma}{\gamma-1}\right)
$$

An input value for $x$ specifies the grid location, and Newton's Method is implemented numerically for rapid convergence of $y$ while satisfying Equation 3.36.

The steady-state solutions of several ZEUS-MP runs at varying grid resolutions 
are shown in Figures 3.8 and 3.9. Each run holds the density and initial velocity fixed in the inner radial ghost zones, which is the standard set of boundary conditions for outflow from a planetary atmosphere with $\dot{M} \ll M$. For each run, $\dot{M}=10^{10} \mathrm{~g} / \mathrm{s}, \gamma=$ 1.1 , and $r_{\mathrm{s}}=10 R_{\mathrm{p}}$. In each figure, the semi-analytic wind solution is obtained by first solving numerically for the wind velocity at a specific grid location $r$ using Equation 3.36 above, and then using Equation 3.26 to return the corresponding density $\rho$, which is the solid line in each panel. The blue, green, and red symbols are for the steady-state grid values using 64, 128, and 256 radial zones, respectively.

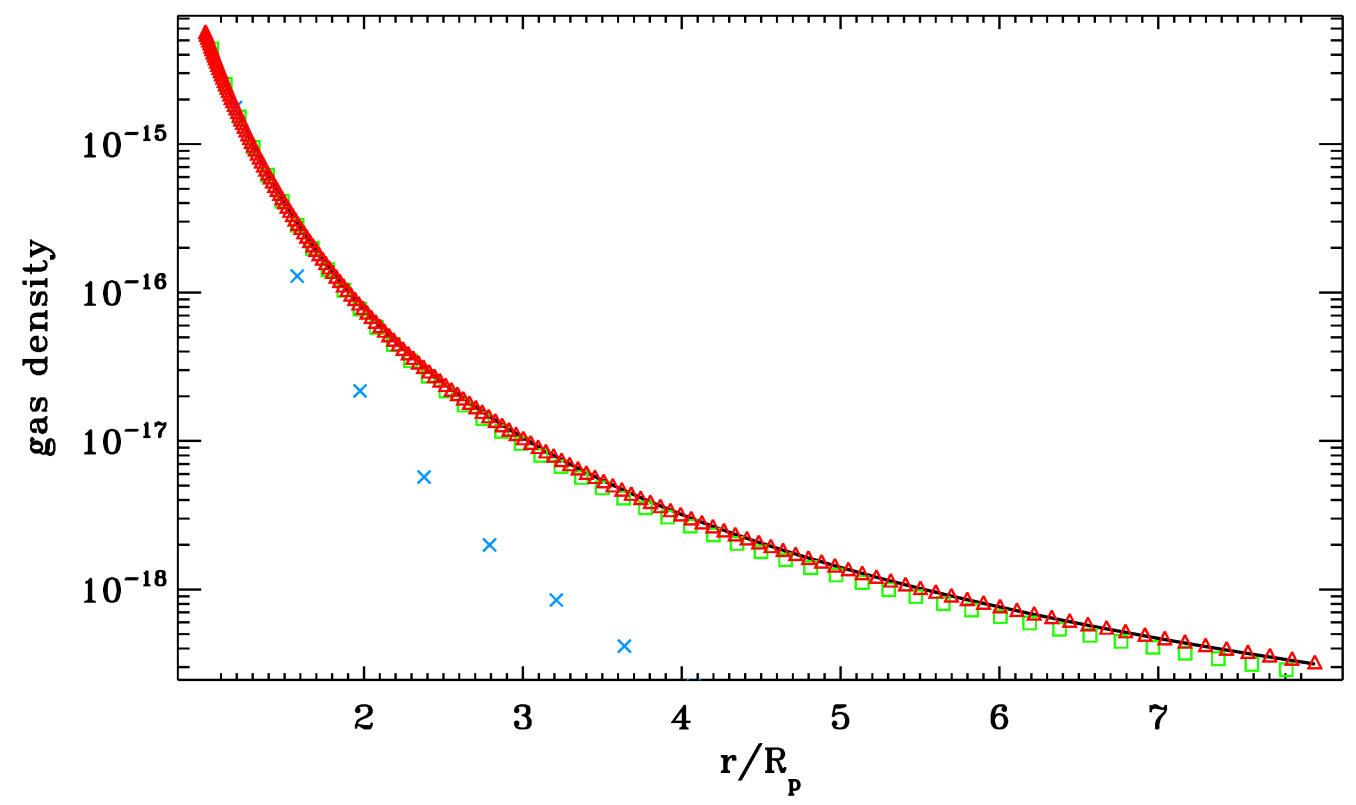

Fig. 3.8. - Radial profiles of the gas density in $\mathrm{g} / \mathrm{cm}^{3}$ at $\theta=\pi / 2$ at three different grid resolutions. Red triangles are 256 radial zones, green squares are 128 radial zones, and blue x's are for 64 radial zones. For comparison, the solid black line is the semi-analytic polytropic wind solution with $\gamma=1.1$ and $\dot{M}=10^{10} \mathrm{~g} / \mathrm{s}$.

A logarithmic grid spacing gives smaller $\delta r / r$ values nearer the inner boundary (i.e., the planet's surface) at $r=R_{p}$, which both figures show is needed for an accurate solution in the wind acceleration region $\left(r<r_{\mathrm{s}}\right)$ near $r=R_{\mathrm{p}}$. These results show the rapid convergence at steady state to the polytropic wind solution at the highest 
resolution (red triangles), where the agreement is best at smaller radii due to the larger radial extent of the the outermost grid zones. These results motivate a radial grid spacing $\delta r / r \sim 0.01$ for a good wind solution for the MHD problem.

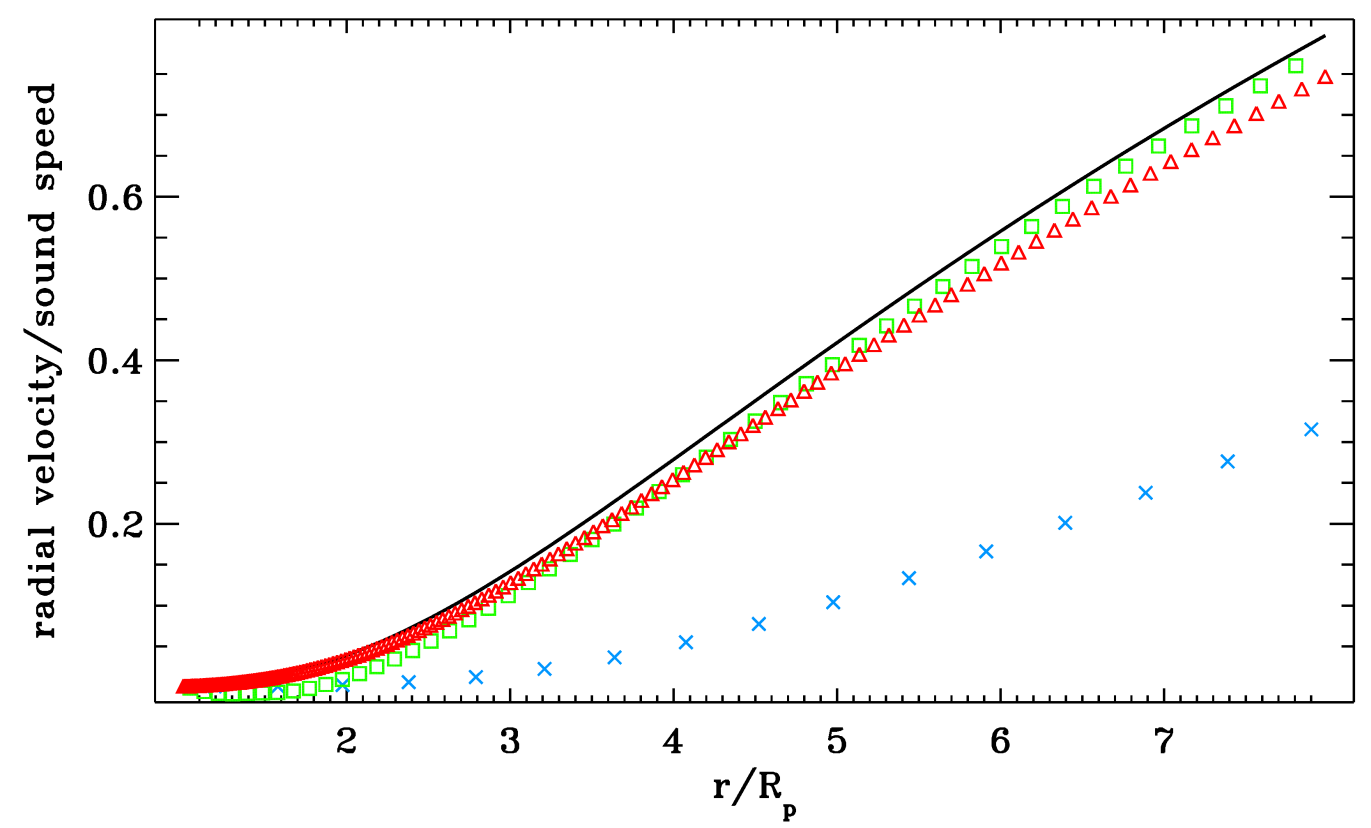

Fig. 3.9. - Radial profiles of the radial velocity normalized by the sound speed at the sonic point, for the same three grid resolutions and parameters as shown in Figure 3.8. The solid line is the polytropic wind solution for comparison.

Given the robustness and stability of the ZEUS-MP MHD code for a wide range of problems, the ZEUS-MP code is a desirable choice to compute the structure of upper atmospheres of HJs. 


\section{Chapter 4}

\section{MHD Simulations of Hot Jupiter}

\section{Upper Atmospheres}

Given the results that I have presented in Chapter 2, and the description of the ZEUS-MP code in Chapter 3, this chapter shows the simulation results for HJ upper atmospheres and he comparison to observations. All of the following results are being prepared for submission to the Astrophysical Journal for publication.

\subsection{Description of the Simulations}

We consider a planet of mass $M_{p}$ and radius $R_{p}$ at orbital distance $D$ from a star of mass $M_{\star}$. The planet's rotation is synchronized to the orbit with angular velocity $\Omega=\left[G\left(M_{\star}+M_{p}\right) / D^{3}\right]^{1 / 2}$, and the spin axis is aligned with the orbital angular momentum. Outside the planet, in the region modeled by the simulations, the gas is not required to corotate with the planet.

Two-dimensional (2D), axisymmetric simulations in spherical coordinates are carried out with the publicly available MHD code ZEUS-MP (see Hayes et al. 2006, and 
references therein), which solves the ideal-MHD equations:

$$
\begin{aligned}
\frac{D \rho}{D t} & =-\rho \nabla \cdot \boldsymbol{v} \\
\rho \frac{D \boldsymbol{v}}{D t} & =-\nabla P+\frac{1}{4 \pi}(\nabla \times \boldsymbol{B}) \times \boldsymbol{B}-\rho \nabla \mathrm{U} \\
\frac{\partial \boldsymbol{B}}{\partial t} & =\nabla \times(\boldsymbol{v} \times \boldsymbol{B}),
\end{aligned}
$$

where the comoving (Lagrangian) derivative is defined as

$$
\frac{D}{D t} \equiv \frac{\partial}{\partial t}+\boldsymbol{v} \cdot \nabla
$$

Equations 4.1-4.3 are the mass continuity, momentum and induction equation, respectively. The quantity $U$ is an effective potential to be defined below, and other symbols have their standard meaning.

Instead of solving the energy equation, an isothermal equation of state, $P=\rho a^{2}$, is used, where $a$ is the (constant) isothermal sound speed. This assumption is equivalent to adding energy to the flow to counter adiabatic cooling, giving rise to a transonic outflow. The isothermal assumption is convenient for the present study, where the focus is not on the initial launching of the wind by gas pressure gradient forces, but rather on magnetic effects. A more detailed study, beyond the scope of this thesis, would include heating and cooling effects in an energy equation. We note, however, that since the magnetic field and rotation are included, the flow is also accelerated in part by the "magneto-centrifugal" effect (Blandford \& Payne 1982), as well as stellar tides.

The computational grid extends from an inner radial boundary at $r=R_{p}$ to the outer boundary at $r=30 R_{p}$, with $R_{p}$ set to the planet's observed transit continuum radius (Southworth 2010), and from the north pole at $\theta=0$ to the south pole at 
$\theta=\pi$. The radial box size was chosen through experimentation so that all MHD critical points in the wind zone were contained within the computational domain for a wide range of model parameters. Our standard resolution is $272 \times 200$, with the radial cell size $\Delta r=r_{i+1}-r_{i}$ increasing outward according to $\Delta r_{i+1} / \Delta r_{i}=1.02 ;$ the ratio was chosen to adequately resolve the wind acceleration region near the base. The $\theta$ grid is uniformly spaced. Surrounding the active grid are two layers of ghost zones at each of the four boundaries used to impose boundary conditions.

At time $t=0$, the fluid is uniformly rotating, $\left(v_{r}, v_{\theta}, v_{\phi}\right)=(0,0, \Omega r \sin \theta)$, and in hydrostatic balance over the entire grid. The tidal and centrifugal forces then pull mass out and initiate the outflow as initially closed field lines now have looptops that are outside the active computational domain. In a reference frame corotating with the planet, and with origin comoving with the planet, hydrostatic balance takes the form (Trammell et al. 2011)

$$
0=-a^{2} \nabla \rho-\rho \nabla U_{\text {rot }}
$$

The potential $U_{\text {rot }}$ includes contributions from the gravity of the planet, the stellar gravity, the dipole term arising from the acceleration of the origin, and the centrifugal force, and takes the form

$$
U_{\mathrm{rot}}(\boldsymbol{x})=-\frac{G M_{p}}{|\boldsymbol{x}|}-\frac{G M_{\star}}{\left|\boldsymbol{x}-\boldsymbol{x}_{\star}\right|}+\frac{G M_{\star} \boldsymbol{x} \cdot \boldsymbol{x}_{\star}}{\left|\boldsymbol{x}_{\star}\right|^{3}}-\frac{1}{2}|\boldsymbol{\Omega} \times \boldsymbol{x}|^{2}
$$

The dipole term, which acts to accelerate the center of mass of the planet, cancels off part of the stellar gravity, leaving only a tidal acceleration. Expressing the position vector in spherical coordinates $\boldsymbol{x}=(r, \theta, \phi)$, the position of the star as 
$\boldsymbol{x}_{\star}=(D, \pi / 2,0)=D \boldsymbol{e}_{x}$, and making the tidal approximation, $r \ll D$ gives

$$
U_{\mathrm{rot}}(\boldsymbol{x}) \simeq-\frac{G M_{p}}{r}-\frac{1}{2} \Omega^{2} r^{2}\left(f_{\mathrm{rot}} \sin ^{2} \theta-1\right)
$$

where the longitude-dependent function $f_{\text {rot }}=1+3 \cos ^{2} \phi$. As the simulations are axisymmetric, we substitute the azimuthal average $\cos ^{2} \phi \rightarrow 1 / 2$, yielding $f_{\text {rot }}=5 / 2$. The Hill radius of the axisymmetrized potential is

$$
r_{\mathrm{H}}=\left(\frac{2 G M_{p}}{3 \Omega^{2}}\right)^{1 / 3} \simeq D\left(\frac{2 M_{p}}{3 M_{\star}}\right)^{1 / 3}
$$

a factor of $2^{1 / 3}$ larger than the physically correct value, which is evaluated along the star-planet line.

Substituting eq.4.7 into eq.4.5, the initial density distribution takes the form

$$
\rho(r, \theta)=\rho_{\mathrm{ss}} \exp \left[-\left(\frac{U_{\mathrm{rot}}(r, \theta)-U_{\mathrm{rot}}\left(R_{p}, \pi / 2\right)}{a^{2}}\right)\right]
$$

where $\rho_{\mathrm{ss}}$ is the density at $(r, \theta)=\left(R_{p}, \pi / 2\right)$. The initial density distribution then requires $a$ and $\rho_{\mathrm{ss}}$ as parameters, in addition to the parameters of the planet, star and orbit.

For simplicity, the simulations are in the non-rotating frame, since moving to the corotating frame would require the addition of Coriolis and centrifugal forces to the momentum equations; however, the origin of the coordinate system still co-moves with the center of the planet. In this reference frame, therfore, the centrifugal term 
can be left out of the potential, giving

$$
\begin{aligned}
U(\boldsymbol{x}) & =-\frac{G M_{p}}{|\boldsymbol{x}|}-\frac{G M_{\star}}{\left|\boldsymbol{x}-\boldsymbol{x}_{\star}\right|}+\frac{G M_{\star} \boldsymbol{x} \cdot \boldsymbol{x}_{\star}}{\left|\boldsymbol{x}_{\star}\right|^{3}} \\
& \simeq-\frac{G M_{p}}{r}-\frac{1}{2} \Omega^{2} r^{2}\left(f \sin ^{2} \theta-1\right),
\end{aligned}
$$

where now $f=1+\cos ^{2} \phi$. The azimuthal average gives $f=3 / 2$ for the non-rotating frame. The $r$ and $\theta$ components of $-\nabla U$ from eq.4.10 are introduced into the ZeusMP code as a source term in the momentum equations. Since the gas is not required to corotate, eq.4.8 may underestimate the radius at which the equatorial acceleration changes sign. An upper limit is found by ignoring the centrifugal force. Using $f=3 / 2$ with eq.4.10 would give $\left(2 G M_{p} / \Omega^{2}\right)^{1 / 3}$ for this radius, larger than the expression in eq. 4.8 by a factor $3^{1 / 3}$.

The initial condition for the magnetic field is a dipole with magnetic axis aligned with the rotation axis:

$$
\begin{aligned}
B_{r} & =B_{0}\left(\frac{r}{R_{p}}\right)^{-3} \cos \theta \\
B_{\theta} & =\frac{B_{0}}{2}\left(\frac{r}{R_{p}}\right)^{-3} \sin \theta \\
B_{\phi} & =0,
\end{aligned}
$$

where the field at the magnetic pole is $B_{0}$. The development of nonzero $B_{\phi}$ at $t>0$ will lead to magnetic torques on the gas and planet. A key parameter of the model is the equatorial value of the plasma $\beta$ at the inner radius:

$$
\beta_{0}=\frac{8 \pi P_{\mathrm{ss}}}{\left(B_{0} / 2\right)^{2}}
$$

where $P_{\mathrm{ss}}=a^{2} \rho_{\mathrm{ss}}$ is the base pressure at the equator, and $B_{0} / 2$ is the magnetic field 
at the equator. This parameter sets the size of the equatorial DZ citepTrammella.

Compared with the initial conditions, the boundary conditions are much more difficult to implement, especially on the inner radial boundary that is the base of both the wind zone and the dead zones. The boundary condition on $\rho$ keeps the inner radial boundary densities in the inner radial ghost zones and the first radial active zones at their initial values for all $t>0$. Although densities in the first active zones are updated at each time step, the updated values are overwritten with their initial values. This guarantees that the base density is held fixed at the prescribed value and provides control of the conditions at the base, even in the wind zone.

For the velocity at the inner radial boundary, $v_{r}=0$ is set at the inner face of the first active zone, as well as in the ghost zones. In other words, $v_{r}\left(R_{p}, \theta\right)=0$ and $v_{r}\left(r^{-}, \theta\right)=0$, where $r^{-}\left(<R_{p}\right)$ denotes the inner radial ghost region. The reflection boundary condition is applied to $v_{\theta}$, so that $v_{\theta}\left(r^{-}, \theta\right)=v_{\theta}\left(r^{+}, \theta\right)$, where $r^{+}$is the symmetry point (with respect to the $r=R_{p}$ surface) in the active domain of the location $r^{-}$in the ghost region.

The boundary condition on the azimuthal velocity component, $v_{\phi}$, is $v_{\phi}\left(r^{-}, \theta\right)=$ $2 v_{\phi}\left(R_{p}, \theta\right)-v_{\phi}\left(r^{+}, \theta\right)$ where $v_{\phi}\left(R_{p}, \theta\right)=\Omega R_{p} \sin \theta$. That is, the average of the first ghost zone and the first active zone should equal the corotation velocity. We have verified that, in the absence of magnetic field, rotation and stellar tides, the inner hydro boundary conditions produce a thermally driven wind that matches, in steady state, the well-known analytic solution.

The magnetic boundary conditions at the inner radial boundary are more complicated to implement. They are enforced through the electromotive force (EMF) $\boldsymbol{\epsilon}=\boldsymbol{v} \times \boldsymbol{B}$, as this will automatically preserve $\partial / \partial t(\nabla \cdot \boldsymbol{B})=0$ during the time evolution. In 2D (axisymmetric) geometry, only the $r$ - and $\theta$-components of $\boldsymbol{\epsilon}$ affect 
$B_{\phi}:$

$$
\frac{\partial B_{\phi}}{\partial t}=\frac{1}{r}\left[\frac{\partial}{\partial r}\left(r \epsilon_{\theta}\right)-\frac{\partial \epsilon_{r}}{\partial \theta}\right] .
$$

Although $B_{\phi}$ is assumed to be zero initially in our simulation, it can grow with time, particularly in the outflow region. The boundary conditions on $\epsilon_{r}$ and $\epsilon_{\theta}$ are designed to enable $B_{\phi}$ in the ghost zones to grow at the same rate as in the active zones. Specifically, we demand

$$
\frac{\epsilon_{r}\left(r^{-}\right)}{r^{-}}=\frac{\epsilon_{r}\left(r^{+}\right)}{r^{+}}
$$

and

$$
\left[\frac{1}{r} \frac{\partial}{\partial r}\left(r \epsilon_{\theta}\right)\right]_{r^{-}}=\left[\frac{1}{r} \frac{\partial}{\partial r}\left(r \epsilon_{\theta}\right)\right]_{r^{+}}
$$

The value of $\epsilon_{\theta}$ in the ghost zone is determined by equation 4.17 together with the condition

$$
\epsilon_{\theta}\left(R_{p}, \theta\right)=\Omega R_{p} \sin \theta B_{r}\left(R_{p}, \theta\right)
$$

which ensures that the footpoints of the magnetic field lines corotate with the planet.

For $\epsilon_{\phi}=v_{r} B_{\theta}-v_{\theta} B_{r}$, the boundary condition $\epsilon_{\phi}\left(R_{p}, \theta\right)=0$ enforces poloidal velocity parallel to poloidal magnetic field at $r=R_{p}$. It also guarantees that $B_{r}(r=$ $R_{p}$ ) remains unchanged, i.e., the footpoints of the field lines are firmly anchored on the rotating inner radial boundary. For $\epsilon_{\phi}$ in the ghost zone, $\epsilon_{\phi}\left(r^{-}\right)=-\epsilon_{\phi}\left(r^{+}\right)$is enforced so that the radial gradient of $\epsilon_{\phi}$, which controls the evolution of $B_{\theta}$, is continuous across the inner radial boundary. This set of magnetic boundary conditions is similar 
to that used successfully by Krasnopolsky et al. (1999) and Krasnopolsky et al. (2003) to simulate protoplanetary disk-driven magnetocentrifugal winds.

The standard "outflow" boundary condition implemented in ZEUS-MP is used at the outer radial boundary, with all hydrodynamic variables and the three components of the EMF projected to zero slope. In addition, $\partial B_{\phi} / \partial t=0$ is set for the outer radial ghost zones, which prevented the growth of unphysically large external currents that sometimes develop near the outer boundary. At the $\theta=0$ and $\theta=\pi$ boundaries, the standard "axial" boundary conditions as implemented in ZEUS-MP is used, which enforces reflection symmetry for the $r$-components of the velocity and the magnetic field (i.e., the $\theta$ - and $\phi$-components are reflected with a change of sign).

The runs typically reach a steady-state solution after a few sound crossing times over the domain, and are stopped after a steady state solution is achieved. A summary of the main model parameters is shown in Tables 4.1 and 4.2.

\subsection{Simulation Results}

A fiducial model is chosen with HD 290458b's parameters and $B_{0}=10 \mathrm{G}$ (Model 1 in Table 4.2). The other simulations listed in Table 4.2 vary the model parameters listed in Table 4.1. Qualitative results will be discussed in $\S 4.2 .1$, and a more quantitative analysis in $\S 4$ 4.2.2. The last subsection ( $\S 4.2 .3$ ) contains a discussion of mass and angular momentum loss rates from the planet.

\subsubsection{Qualitative Results: Magnetic Field and Tidal Strength}

One of the most important qualitative results of this thesis is the confirmation of the three-zone structure of the magnetosphere predicted analytically in Trammell et al. (2011). The three distinct regions are clearly visible in Figures 4.1 and 4.2 - (1) an 
Table 4.1. Primary Model Parameters

\begin{tabular}{lcc}
\hline \hline Parameter & Range & Description \\
\hline$R_{p}$ & $1.35 R_{\mathrm{Jup}}$ & planet radius \\
$\rho_{\mathrm{Ss}}$ & $\sim 10^{-16}-10^{-13} \mathrm{~g} \mathrm{~cm}^{-3}$ & substellar point mass density \\
$a$ & $9-11 \mathrm{~km} \mathrm{~s}^{-1}$ & isothermal sound speed \\
$B_{0}$ & $1.0-100 \mathrm{G}$ & polar magnetic field strength \\
$M_{p}$ & $0.7 M_{\mathrm{Jup}}$ & planet mass \\
$M_{\text {star }}$ & $1.1 M_{\odot}$ & host stellar mass \\
$D$ & $0.035-0.06 \mathrm{AU}$ & orbital separation \\
\hline \hline
\end{tabular}

Note. - Description and range of the model parameters used in the simulations.

Table 4.2. Parameters for HD 209458b Runs

\begin{tabular}{cccccc}
\hline \hline Run & $D(\mathrm{AU})$ & $P_{s}(\mu \mathrm{bar})$ & $a(\mathrm{~km} / \mathrm{s})$ & $B_{0}(\mathrm{G})$ & $\beta_{0}$ \\
\hline Model 1 & 0.047 & 0.05 & 10.0 & 10.0 & 0.051 \\
Model 2 & 0.047 & 0.05 & 10.0 & 1.0 & 5.1 \\
Model 3 & 0.047 & 0.05 & 10.0 & 50.0 & 0.002 \\
Model 4 & 0.047 & 0.05 & 9.0 & 10.0 & 0.041 \\
Model 5 & 0.047 & 0.05 & 11.0 & 10.0 & 0.061 \\
Model 6 & 0.047 & 0.005 & 10.0 & 10.0 & 0.0051 \\
Model 7 & 0.047 & 0.5 & 10.0 & 10.0 & 0.51 \\
Model 8 & 0.035 & 0.05 & 10.0 & 10.0 & 0.051 \\
Model 9 & 0.06 & 0.05 & 10.0 & 10.0 & 0.051 \\
\hline
\end{tabular}

Note. - This table contains simulation parameters varying a single parameter $\left(B_{0}, D, a, P_{\mathrm{ss}}\right)$ relative to the fiducial case (Model 1). The planetary radius and mass are fixed to $R_{p}=$ $1.35 R_{J}, M_{p}=0.7 M_{J}$. The values of $\beta_{0}$ can be compared to the value $\beta_{0, \mathrm{Jup}}=0.069$ using Jupiter's magnetic field and a base pressure $P_{\mathrm{ss}}=0.05 \mu \mathrm{bar}$. 


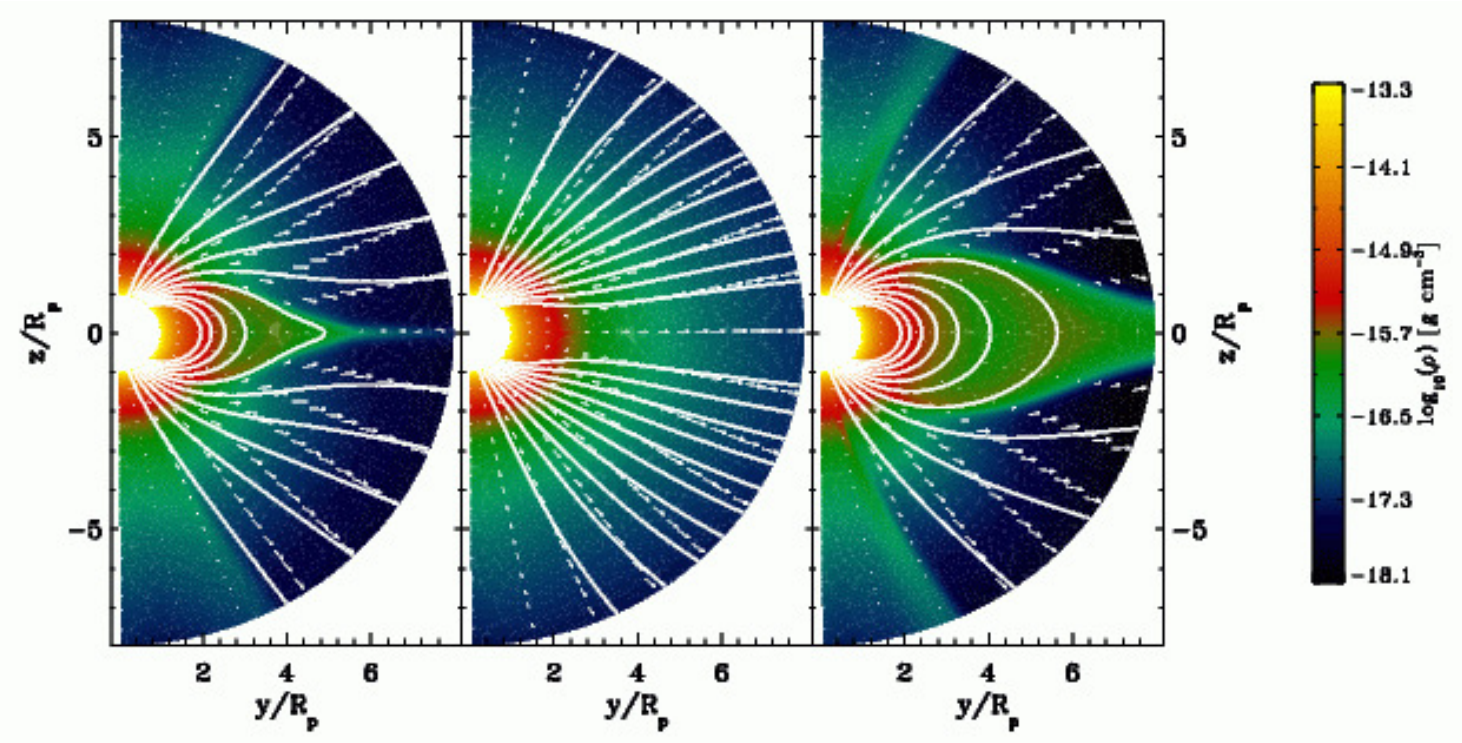

Fig. 4.1.- Contours of total gas density $\rho(r, \theta)$ in units of $\mathrm{g} \mathrm{cm}^{-3}$ as viewed during transit, illustrating the effect of the magnetic field (white lines) on the size of the equatorial DZ. Left: Model 1 (fiducial model with $B_{0}=10 \mathrm{G}$ ) Center: Model 2 $\left(B_{0}=1 \mathrm{G}\right)$ Right: Model $3\left(B_{0}=50 \mathrm{G}\right)$. For these three models, the remaining parameters given in Table 4.2 are otherwise identical. White arrows indicate the direction and magnitude of the poloidal fluid velocity. Only the inner $8 R_{p}$ portion of the grid is shown (the magnetic field lines near the planet are not drawn for clarity).

equatorial dead-zone (DZ) containing static gas confined by the magnetic field, (2) a wind-zone (WZ) where an outflow is driven along open magnetic field lines, and (3) a second polar DZ where the stellar tide has shut off the outflow (see also Fig. 7 of Trammell et al. 2011). The range of magnetic field and stellar tide over which the equatorial and polar DZ's exist has been discussed in Trammell et al. (2011). Roughly, the equatorial DZ requires $\beta_{0} \lesssim 1$, i.e. the magnetic pressure dominates gas pressure at the base of the atmosphere (the hydrogen ionization zone) at the equator. The existence of the polar dead zone, and the inability to drive a transonic outflow there, occurs inside a critical orbital separation. Roughly, this criterion translates into the rotation velocity $\Omega$ at the fiducial sonic point radius $r_{\mathrm{s}, 0}=G M_{p} / 3 a^{2}$ must 


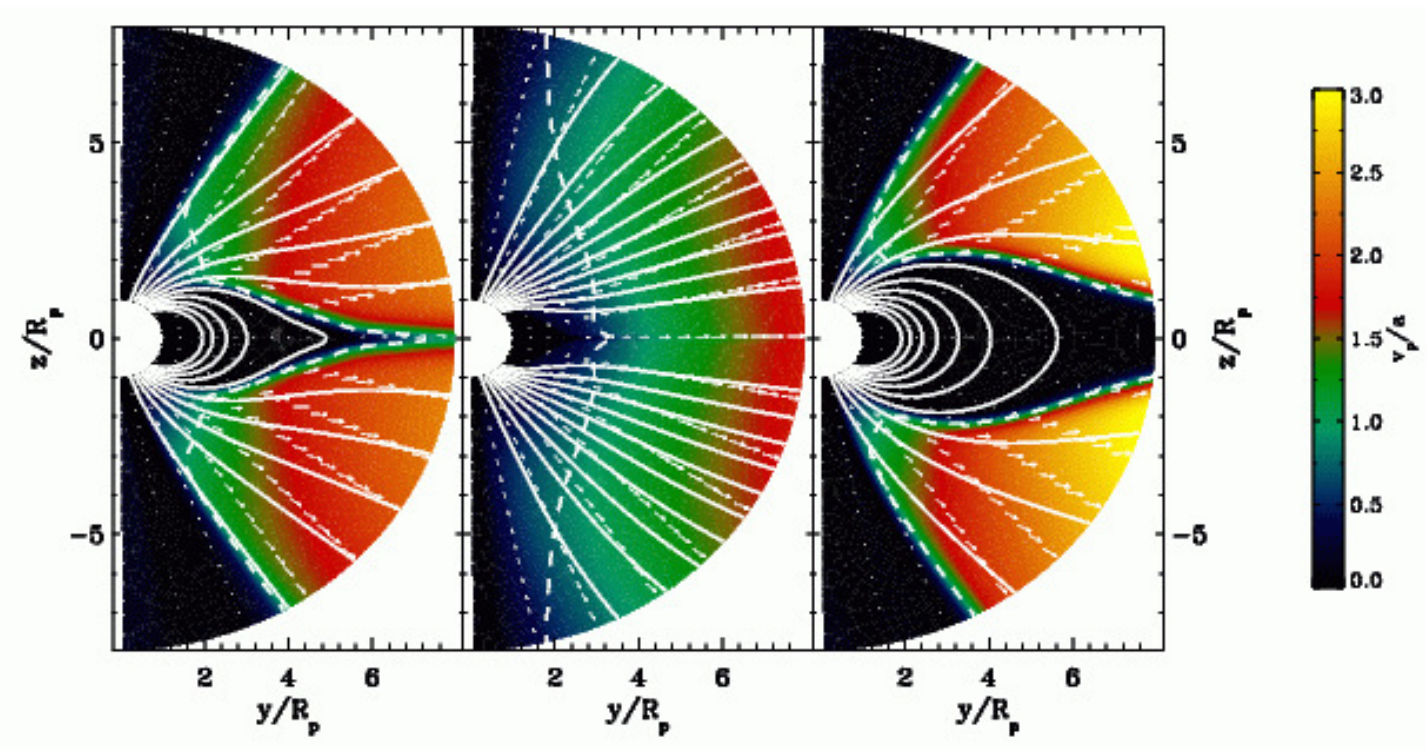

Fig. 4.2.- Similar to Figure 4.1, but for contours of poloidal velocity $v_{p}$ in units of the isothermal sound speed $a$. Left: Model 1 (fiducial model) Center: Model 2 Right: Model 3 for parameters given in Table 4.2. White-dashed contours trace the (slow magneto-)sonic points, which trace the vicinity of the shear layers separating the static DZs (i.e., darkest contours) from the transonic WZs.

be supersonic, $\Omega r_{\mathrm{s}, 0} \gtrsim a$.

The DZ-WZ boundaries in the simulation contain a shear layer separating the outflowing gas in the WZ from the static gas in the DZ. In addition, the magnetic field changes rapidly in this boundary layer, implying a current sheet. The origin of this current sheet is that, for identical Bernoulli constant at the inner boundary, the WZ has smaller density compared to the neighboring DZ by a factor $\sim \exp \left(-v^{2} / a^{2}\right)$ (Mestel \& Spruit 1987; Trammell et al. 2011), where $v$ is the poloidal wind speed. Since the total pressure, gas plus magnetic, must be continuous across the boundary, the decrease in gas pressure implies an increase in magnetic pressure, and hence a current sheet. Numerical issues related to the shear in velocity and magnetic field will be discussed further in the Appendix.

Figure 4.1 illustrates the effect of the magnetic field on the density profile in the 


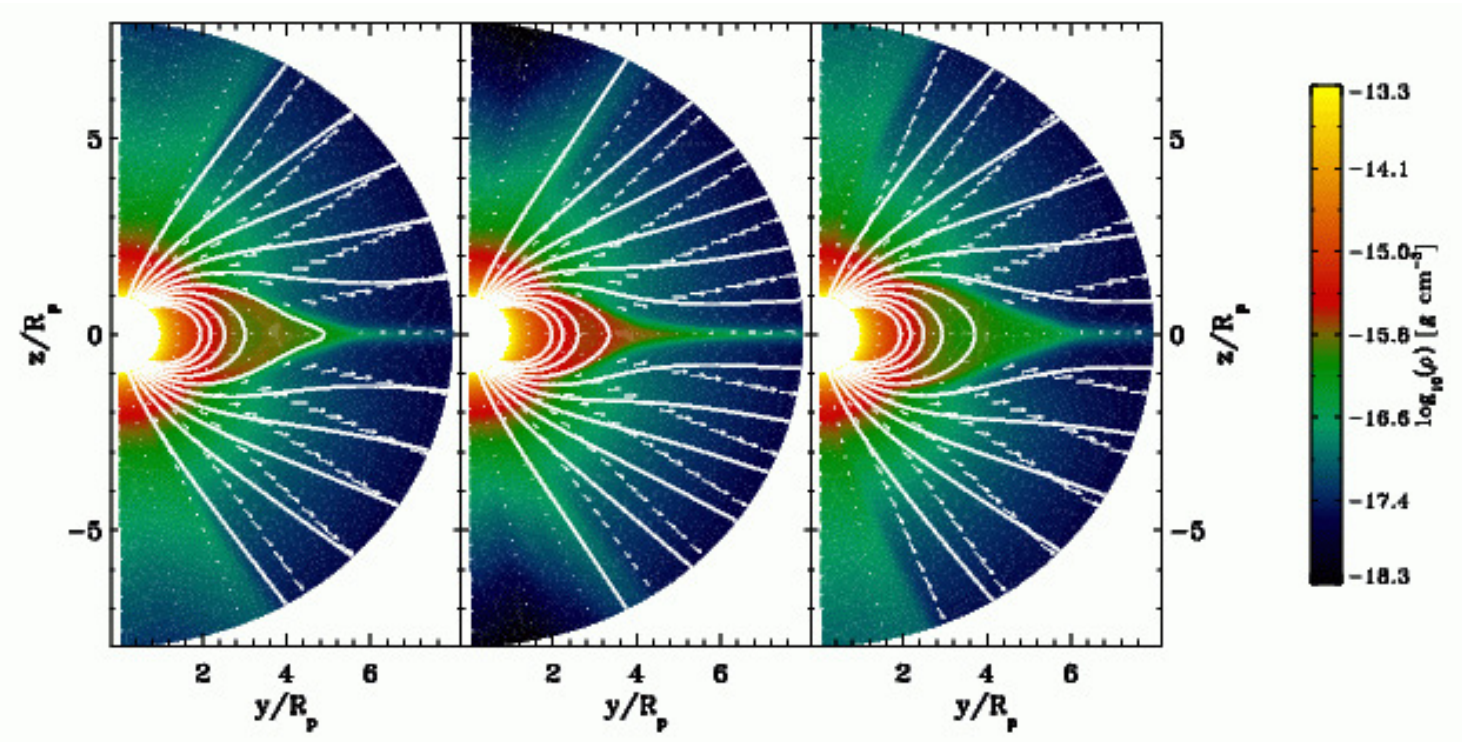

Fig. 4.3.- Similar to Figure 4.1 illustrating how the stellar tide influences the size of the equatorial/polar DZs. All panels have the same parameters as Model 1 of Table 4.2 , except for the varying orbital distance $D$. Left: the fiducial Model 1 for HD 209458b with $D=0.047$ AU. Center: Model 8 ( $D=0.035$ AU), which illustrates the effect of increasing the stellar tide by shrinking the planet's orbit. Right: Model $9(D=0.06 \mathrm{AU})$. Note that the polar DZ size is larger and equatorial DZ small for the stronger tide case (middle panel), as can be seen by the range of angles with zero-length velocity vectors (white arrows). As in Figure 4.1, the innermost magnetic field lines have been suppressed for clarity.

magnetosphere. The parameters for the runs in each panel are identical except for the magnetic field, with $B_{0}=10,1$ and $50 \mathrm{G}$ from left to right, respectively (Models 1-3 in Table 4.2). As predicted in Trammell et al. (2011) and expected intuitively, the size of the equatorial DZ increases with the field strength, when all other parameters are held fixed. For a dipole field with footprint at $\theta_{0}$, the magnetic pressure decreases outward along the field line as $B^{2} \propto \sin ^{12} \theta_{0} / \sin ^{12} \theta$, and so field lines nearer the pole, with smaller $\theta_{0}$, suffer a larger decrease in magnetic pressure from pole to equator. The larger DZ size for larger $B_{0}$ then reflects the inability of ram pressure to overcome magnetic pressure, except in a smaller region near the pole where the field decreases 


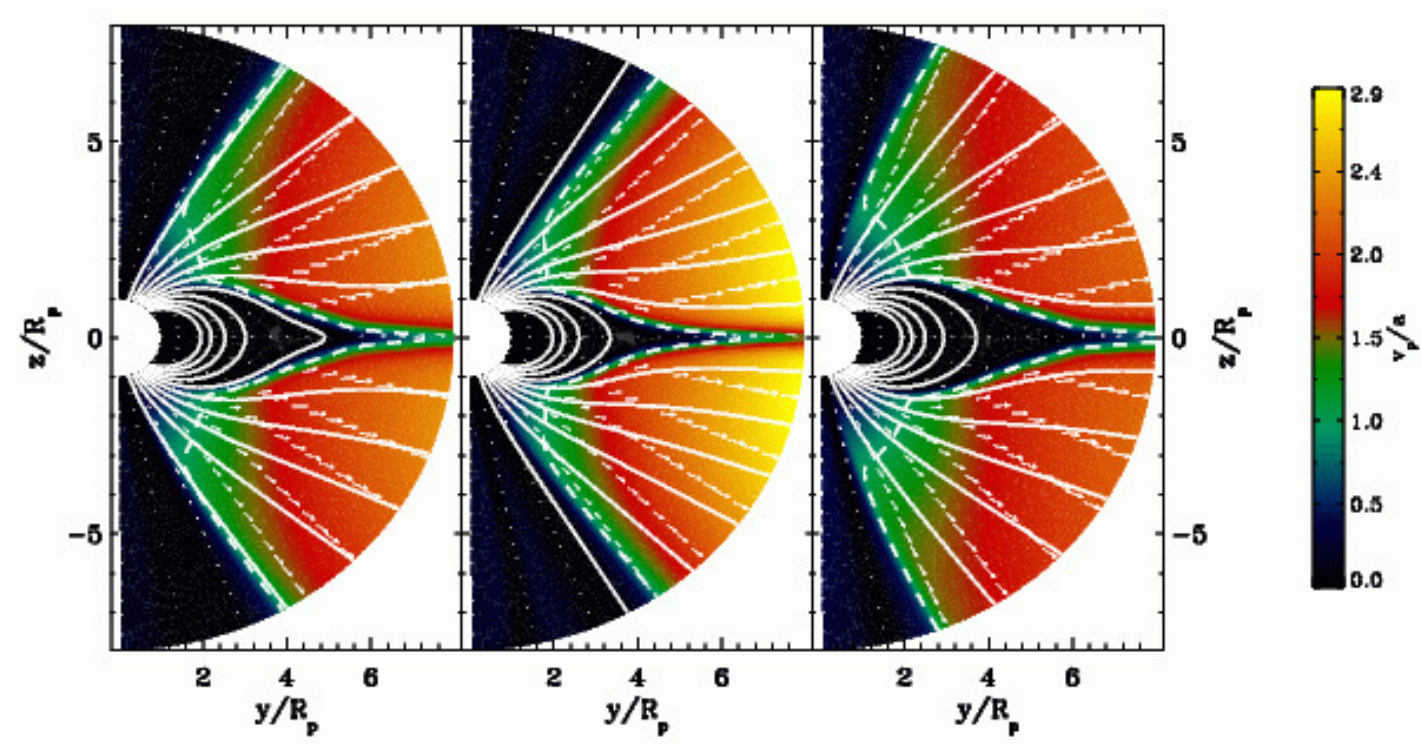

Fig. 4.4.- Similar to Figure 4.3 showing the $2 \mathrm{D}$ structure of the poloidal velocity $v_{p}$ field, in units of the isothermal speed $a$ for the same three models shown in Figure 4.3. Higher stellar tide pushes the slow magnetosonic point inward at mid-latitudes and leads to a stronger outflow ram pressure, which can open a larger region of the planetary magnetic field lines and decrease the size of the equatorial DZ.

outward more rapidly.

The observational implication of the increase of DZ size with magnetic field is that more of the circum-planetary material is expected to be confined within the static dead zone, which should make this region easier to probe with transit observations (see section 4.3). A weaker magnetic field $B_{0} \ll 1 \mathrm{G}$ would not significantly confine the gas, and a larger range of latitudes will participate in the outflow.

Except for the weakest field case, the equatorial DZ dominates the region near the planet, with a higher density than the neighboring WZ. For example, at a distance $r \simeq 6 R_{p}$, or roughly twice the Roche Lobe distance for HD 209458b in the fiducial Model 1 (left panel of Figure 4.1), the gas density is a factor of $\sim 9$ higher than in the neighboring $\mathrm{WZ}$ at the same radius. The density jump is less dramatic in the polar DZ, where the wind has been shut off by the stellar tide. The fluid there 
is nearly hydrostatic, with a pressure scale height determined by the potential in Equation 4.10. Its density decreases more slowly with radius than in the WZ, despite the stronger inward gravity in the DZ. For Model 1, the density jump across in the polar DZ/WZ boundary is relatively modest $\left(\sim 3\right.$ at a distance $\left.r \simeq 6 R_{p}\right)$.

To more clearly differentiate the polar/equatorial DZs from the neighboring WZ, we plot in Figure 4.2 the poloidal velocity for the same Models 1-3 shown in Figure 4.1. The more dramatic contrast between the static DZ regions, where the fluid velocity is very subsonic, and the WZ with transonic outflow further illustrates the existence of the polar/equatorial DZs. The changing colors show the accelerating outflow in the mid-latitude regions along open magnetic field lines that have been combed out by currents in the magnetosphere. The darkest regions can be compared to the highest density regions in Figure 4.1.

Besides the field strength $B_{0}$, the structure of the magnetosphere is also influenced by the stellar tide. The tidal effects are illustrated in Figures 4.3 and 4.4. The left panel is the fiducial Model 1. In the middle panel (Model 8), the orbital distance has been decreased to $D=0.035 \mathrm{AU}$, so that the stellar tide is stronger than that for HD 209458b ( $D=0.047 \mathrm{AU})$. As predicted in Trammell et al. (2011), the stronger stellar tide increases the outward acceleration of the mid-latitude outflow by moving the sonic point inward. It results in an equatorial DZ that is slightly smaller in size but denser at the same distance from the planet relative to Model 1. Figure 4.4 shows that the polar DZ size is also larger for the stronger tide case, as can be seen by the range of angles occupied by largely subsonic gas with small poloidal velocities, again in broad agreement with the analytic results of Trammell et al. (2011) 


\subsubsection{Quantitative Analysis: Density and Velocity Profiles}

To examine the numerical simulations in more detail, Figure 4.5 shows the run of density and poloidal velocity along three different co-latitudes for the fiducial Model 1. The $\theta$ values are chosen to highlight the separate polar DZ, WZ and equatorial DZ regions, respectively. Along $\theta \approx 0$, near the pole, the density decreases rapidly with $r$ because the downward gravity of the planet and star must be balanced by pressure gradient in hydrostatic equilibrium. For this region, the flow speed remains well below the sound speed, in agreement with Paper I, which predicts the absence of a transonic solution in the polar region. The $\theta \approx \pi / 4$ line initially passes through the equatorial dead zone (where the poloidal velocity is close to zero; see the second panel of Figure 4.5), before entering the wind zone. In the wind zone, the flow speed increases away from the planet, reaching nearly twice the sound speed at $r=8 R_{p}$. The density drops with distance accordingly.

The density distribution along the equator at $\theta \approx \pi / 2$ is the most intriguing. After an initial rapid decrease, it increases for a short distance near $r \simeq 3 R_{p}$, and then resumes a slow decline. Such a "bump" in the density profile was predicted in Trammell et al. (2011), for the gas outside the Hill radius, yet still confined inside the static magnetosphere. This is a consequence of the outward pointing gravity outside the Hill radius in eq.4.8, causing the density to increase outward instead of inward. However, the outward increase in figure 4.5 occurs well inside $r_{\mathrm{H}}$ ! Hence it cannot be due to the change in the sign of gravity. In the Appendix the origin of this density increase is explored, and seems to be due to viscous stresses associated with numerical effects near the equatorial DZ/WZ boundary. As the numerical resolution is increased, the density bump in figure 4.5 decreases in size. We show in the Appendix that increasing the resolution has a $\lesssim 1 \%$ on the integrated transit depth, even as the 
spurious density bump decreases. This gives confidence that resolution-dependent effects are not leading to large errors in the transit depth.

\subsubsection{Mass and Angular Momentum Loss Rates}

The MHD simulations presented in this paper allow a more accurate determination of the rates of mass and angular momentum losses $(\dot{M}$ and $\dot{J})$ as compared to the semianalytic solutions from Trammell et al. (2011), since here the magnetic field geometry and fluid velocity are self-consistently computed. These quantities are computed as a function of $r$ by integrals over $\theta$ :

$$
\dot{M}(r)=2 \pi r^{2} \int_{0}^{\pi} d \theta \sin \theta \rho(r, \theta) v_{r}(r, \theta)
$$

and

$$
\begin{aligned}
\dot{J}(r) & =2 \pi r^{3} \int_{0}^{\pi} d \theta \sin ^{2} \theta \\
& \times\left[\rho(r, \theta) v_{r}(r, \theta) v_{\phi}(r, \theta)-\frac{B_{r}(r, \theta) B_{\phi}(r, \theta)}{4 \pi}\right] .
\end{aligned}
$$

Typically $\dot{M}(r)$ and $\dot{J}(r)$ are constant with radius to better than $1 \%$, which provides a check on the accuracy of the numerical solutions. Table 4.3 summarizes the results for $\dot{M}$ and $\dot{J}$ for Models 1-9.

The planet's magnetic field affects the dynamics in several ways. A stronger magnetic field increases the size of the equatorial DZ, restricting the WZ to a smaller range of latitudes. Therefore, one might expect that the mass-loss rate will decrease for a stronger magnetic field. This expectation is born out in the $\dot{M}$ values presented in Table 4.3 , where the mass loss decreases by $\sim 35 \%$ for a factor of 5 increase in $B_{0}$ from Model 1 to Model 3. Despite the reduction in $\dot{M}$, the total angular momentum 
loss rate $\dot{J}$ increased by a factor of $\sim 2.5$, implying an increase in loss of specific angular momentum, $\dot{J} / \dot{M}$, due to a longer magnetic lever arm for the torque. The effect of the magnetic field and tides on the specific angular momentum loss is most clearly displayed in column 4 of Table 4.3 . The quantity $\dot{J} /\left(\dot{M} \Omega R_{\mathrm{p}}^{2}\right)$ has the value $2 / 3$ ignoring these effects (Mestel 1968), but is significantly larger here, even for relatively weak field cases. Conversely, for a weaker magnetic field (i.e., Model 2), $\dot{M}$ is larger due to the larger range of latitudes in the WZ (see the center panel of Figure 4.2).

Stronger tides result in a slightly smaller equatorial DZ, because the outward tidal force can open more magnetic field lines, but a larger polar DZ, due to the increased potential barrier. Stronger tide also moves the sonic point inward, which tends to increase $\dot{M}$. For example, $\dot{M}$ of the stronger tide Model 8 is increased slightly, by a factor of $\simeq 20 \%$, compared to Model 1 . Presumably if the tide is increased to the point that the sonic point moves all the way into the steeply rising density profile deeper in the atmosphere (figure 4.5) will result in a greater sensitivity to the strength of the tide, as is expected for Roche lobe overflow.

A much larger change in $\dot{M}$ comes from varying the base pressure $P_{s s}$ (Models 6 and 7) or the isothermal sound speed $a$ (Models 4 and 5). For example, when $P_{s s}$ increases by a factor of 10 , from 0.05 to $0.5 \mu \mathrm{bar}, \dot{M}$ rises by a factor of 16.9 . When $a$ increases by $10 \%$, from 10 to $11 \mathrm{~km} / \mathrm{s}, \dot{M}$ shoots up by a factor of 4.16 ! In the more heavily mass-loaded winds, the field lines bend backward significantly in the azimuthal direction relatively close to the planet, forcing the fluid to rotate substantially below the corotation speed. The self-consistent treatment of the deviation from corotation here is an improvement over the analytic solutions of Trammell et al. (2011). Conversely, a smaller $P_{s s}$ or a leads to a lower $\dot{M}$, and a wind that is dominated by the magnetic field out to a larger distance. It is interesting to note that ratio 
$\dot{J} /\left(\dot{M} \Omega R_{p}^{2}\right)$ has rather large values of 404.68 and 455.36 for Model $4(a=9 \mathrm{~km} / \mathrm{s})$ and $6\left(P_{s s}=0.005 \mu\right.$ bar $)$, respectively. They are very different from the purely hydro winds from the planet, where the ratio is $2 / 3$. The relatively low mass loss rate in these cases allows the magnetic field to effectively enforce corotation up to a distance of $\sim 20 R_{p}$.

The large spin-down torques found in the strongly magnetized models may torque the planet away from synchronous rotation Trammell et al. (2011) Defining $\Gamma=$ $\dot{J} /\left(\dot{M} \Omega R_{\mathrm{p}}^{2}\right)$ and $J=\alpha M R_{\mathrm{p}}^{2} \Omega$, the spindown timescale is

$$
\begin{aligned}
\frac{J}{\dot{J}} & =\frac{\alpha}{\Gamma}\left(\frac{M}{\dot{M}}\right) \\
& \simeq 3 \times 10^{7} \mathrm{yr}\left(\frac{\alpha}{0.1}\right)\left(\frac{660}{\Gamma}\right)\left(\frac{M}{0.7 M_{\mathrm{J}}}\right)\left(\frac{2 \times 10^{11} \mathrm{~g} \mathrm{~s}^{-1}}{\dot{M}}\right)
\end{aligned}
$$

for Model 3 parameters. Deviations from synchronous rotation depend on the planet's tidal $Q$ (e.g. Wu \& Murray 2003). As a numerical example, a tidal synchronization timescale $\sim 10^{6}$ yr implies deviations at the level $3 \%$ from synchronous rotation, based on the ratio of the magnetic torque spindown and tidal synchronization timescales. This persistent asynchronous rotation implies a gravitational tide heating rate of $\dot{E}_{\mathrm{gt}} \simeq 0.03 \Omega \dot{J} \simeq 10^{23} \mathrm{erg} \mathrm{s}^{-1}$ for optimistic parameters. This energy input is small compared to the cooling luminosity of Jupiter, $3 \times 10^{24} \mathrm{erg}$, and is not expected to significantly alter the cooling history for HD 209458b. However, in torque equilibrium, and with all else held fixed, $\dot{E}_{\text {heat }} \propto D^{6}$, so that strongly magnetized planets out near $D \sim 0.1$ AU would be heated at a level $\simeq 10^{25} \mathrm{erg}$, which may inflate the hypothetical planet by $\sim 0.1-0.2 R_{\mathrm{J}}$, based on the cooling rates presented in Arras \& Socrates (2009). 
Table 4.3. Mass/Ang. Mom. Loss Rates (HD 209458b)

\begin{tabular}{ccccc}
\hline \hline Run & $\dot{M}$ & $\dot{J}$ & $\dot{J} /\left(\dot{M} \Omega_{p} R_{p}^{2}\right)$ & $\delta F / F$ \\
\hline Model 1 & 3.29 & 10.20 & 162.24 & 0.100 \\
Model 2 & 6.12 & 1.17 & 9.97 & 0.125 \\
Model 3 & 2.11 & 26.40 & 655.64 & 0.157 \\
Model 4 & 0.48 & 3.74 & 404.68 & 0.048 \\
Model 5 & 13.70 & 19.05 & 72.81 & 0.209 \\
Model 6 & 0.25 & 2.20 & 455.36 & 0.028 \\
Model 7 & 55.50 & 37.59 & 35.46 & 0.470 \\
Model 8 & 3.89 & 15.24 & 131.92 & 0.154 \\
Model 9 & 3.21 & 7.04 & 165.70 & 0.088 \\
\hline \hline
\end{tabular}

Note. - Mass-loss rates $\dot{M}\left[10^{11} \mathrm{~g} / \mathrm{s}\right]$ and angular momentum loss rates $\dot{J}\left[10^{28} \mathrm{~g} \mathrm{~cm}^{2} / \mathrm{s}\right]$ for the 9 models with parameters specified in Table 4.2 , along with the corresponding integrated Ly $\alpha$ transit depth $\delta F / F$ (see Equation 4.26) from 200 to $+200 \mathrm{~km} \mathrm{~s}^{-1}$ from line center. 


\subsection{Transit Depths in $\operatorname{Ly} \alpha$}

Section 4.2 described numerical solutions for the MHD variables $\rho$ and $\boldsymbol{v}$ for different model parameters. In this section the mass density $\rho$ is converted into atomic hydrogen number density $n_{\mathrm{H}}$, and the transmission spectra for the models in Table 4.2 are discussed.

As a point of departure when considering transmission spectra of the MHD simulation results, we summarize the simple model of Lecavelier Des Etangs et al. (2008). They consider a plane parallel, isothermal atmosphere with base radius $R_{\mathrm{b}}$ and altitude $z=r-R_{\mathrm{b}}$. The number density is then $n(z)=n_{0} \exp (-z / H)$, where $H=k_{b} T /\left(\mu m_{p} g\right)$ is the scale height, $\mu$ is the mean molecular weight, and $g=G M_{\mathrm{p}} / r^{2}$. The path length through the atmosphere is $\ell \simeq \sqrt{2 \pi R_{\mathrm{b}} H}$, giving an optical depth $\tau_{\nu}(z)=n_{0} \sigma_{\nu} \ell \exp (-z / H)$. Setting $\tau_{\nu}\left(z_{\nu}\right)=1$ gives the altitude

$$
z_{\nu} \simeq H \ln \left(\frac{1}{n_{0} \sigma_{\nu} \sqrt{2 \pi R_{\mathrm{b}} H}}\right)
$$

up to which the atmosphere is optically thick. The transit depth is then

$$
\frac{R_{\mathrm{p}}^{2}(\nu)}{R_{\star}^{2}} \simeq \frac{R_{\mathrm{b}}^{2}+2 R_{\mathrm{b}} z_{\nu}}{R_{\star}^{2}} .
$$

The altitude $z_{\nu} \propto H \propto T / \mu g$, so hot atmospheres of low mean molecular weight gas around planets with low gravity will have large scale heights and transit depths. Due to the steeply falling density, the transit depth has only a weak logarithmic dependence on $\sigma_{\nu}$.

For the Ly $\alpha$ transit depths of the MHD models considered here, the DZ is hydrostatic, but the tidal/rotational forces are important, and so gravity is weaker than $G M_{\mathrm{p}} / r^{2}$. The corresponding larger scale heights make the plane parallel limit inac- 
curate, and the density profile, even of isothermal models, tends not to fall as steeply as it does deeper in the atmosphere. Hence for careful work numerical integrations are required. However, the analytic model gives useful intuition and is simple.

\subsubsection{Computing the Transit Profiles}

Stellar Ly $\alpha$ of passing through the planet's atmosphere can be absorbed or scattered out of the line of sight to the observer, causing a decrease in flux. In addition, the interstellar medium (ISM) can absorb/scatter the light, most prominently in the Doppler core of the line. The spectrum observed at Earth is the combination of these two effects. If the in-transit flux is $F_{\nu}$ and the out-of-transit flux is $F_{\nu}^{(0)}$, the fractional decrease in flux, the transit depth, is $\left(F_{\nu}^{(0)}-F_{\nu}\right) / F_{\nu}^{(0)}$.

The optical depth through the planet's atmosphere is given by

$$
\tau_{\nu}(y, z)=\int d x n_{H}(x, y, z) \sigma_{\nu}(x, y, z)
$$

where $n_{\mathrm{H}}$ is the number density of the atomic hydrogen in the 1s state, $x$ specifies the direction along the line of sight to the star, $y$ and $z$ are the perpendicular coordinates on the sky, and $\sigma_{\nu}$ is the $\operatorname{Ly} \alpha(1 \mathrm{~s} \rightarrow 2 \mathrm{p})$ cross section. This line profile is taken to be a Voigt function (e.g. Rybicki \& Lightman 1979) evaluated using the isothermal temperature $T$, and bulk fluid velocity is included by transforming the photon frequency from the planet frame to the rest frame of the fluid.

The transit depth will be expressed in terms of a frequency dependent planet radius, $R_{\mathrm{p}}(\nu)$, which is defined as the radius of an opaque disk that is required to 
produce the same transit depth as the integral over the model atmosphere:

$$
\frac{F_{\nu}^{(0)}-F_{\nu}}{F_{\nu}^{(0)}} \equiv \frac{R_{\mathrm{p}}^{2}(\nu)}{R_{\star}^{2}}=\int_{\mathrm{star}} d y d z\left[1-e^{-\tau_{\nu}(y, z)}\right]
$$

where corrections due to limb darkening have been ignored for simplicity. The fractional decrease in flux in eq.4.25 is independent of ISM absorption, and depends solely on the planetary atmosphere. The integration over y and $\mathrm{z}$ extends over the stellar disk, where star has radius $R_{\star}$.

The frequency-integrated transit depth for the models is calculated as

$$
\frac{\delta F}{F}=\frac{\int d \nu I_{\nu}^{(\star)}\left(\frac{R_{\mathrm{p}}(\nu)}{R_{\star}}\right)^{2} e^{-\tau_{\nu}^{(\mathrm{ISM})}}}{\int d \nu I_{\nu}^{(\star)} e^{-\tau_{\nu}^{(\mathrm{ISM})}}}
$$

where

$$
I_{\nu}^{(*)}=\left[1+\left(\frac{\Delta v}{67 \mathrm{~km} \mathrm{~s}^{-1}}\right)^{3}\right]^{-1}
$$

is a fit to the shape of the $\operatorname{Ly} \alpha$ intensity of the Sun under quiet solar conditions (Feldman et al. 1997). The limits of integration in Equation 4.26 are $-200 \mathrm{~km} \mathrm{~s}^{-1} \leq$ $\Delta \mathrm{v} \leq 200 \mathrm{~km} \mathrm{~s}^{-1}$ as in Ben-Jaffel (2008). The ISM optical depth $\tau_{\nu}^{(\mathrm{ISM})}$ is computed using the Voigt line profile evaluated with a temperature $T_{\text {ism }}=8000 \mathrm{~K}$ and a neutral hydrogen column $N_{\mathrm{H} \text {,ism }}=10^{18.4} \mathrm{~cm}^{-2}$ (Wood et al. 2005). The Ly $\alpha$ line is completely absorbed within $\Delta v=c\left(\nu-\nu_{0}\right) / \nu_{0} \simeq \pm 50 \mathrm{~km} / \mathrm{s}$ from line center by the ISM.

The HI number density $n_{H}(x, y, z)$ is computed by assuming a balance between optically-thin photoionization and radiative recombination,

$$
J_{0} n_{\mathrm{H}}=\alpha n_{\mathrm{e}} n_{\mathrm{p}}
$$


where $J_{0} \approx(6 \mathrm{hr})^{-1}(0.047 \mathrm{AU} / D)^{2}$ is the ionization rate for a Solar EUV spectrum (Trammell et al. 2011), and $\alpha_{B}(T) \simeq 2.6 \times 10^{-13} \mathrm{~cm}^{3} \mathrm{~s}^{-1}\left(10^{4} \mathrm{~K} / T\right)^{0.8}$ is the case $\mathrm{B}$ radiative recombination rate (Osterbrock \& Ferland 2006). Assuming charge neutrality, $n_{e}=n_{p}$, and setting $\rho=m_{p}\left(n_{\mathrm{p}}+n_{\mathrm{H}}\right)$, eq.4.28 has the analytic solution

$$
n_{H}=\left[\frac{\sqrt{J_{0} / \alpha_{B}+4 \rho / m_{p}}-\sqrt{J_{0} / \alpha_{B}}}{2}\right]^{2}
$$

At a number density $n_{\text {eq }}=J_{0} / \alpha_{B}$ the gas at density $\rho$ is $50 \%$ ionized with $n_{H}=n_{p}$. For $n_{\mathrm{H}} \gtrsim n_{\text {eq }}$, the gas is mostly neutral, and vice versa for $n_{\mathrm{H}} \lesssim n_{\text {eq }}$. The use of a constant $J_{0}$ above simplifies the problem by requiring only the local gas density $\rho$ to evaluate $n_{H}$.

\subsubsection{Results for HD 209458b}

Figure 4.6 compares the $\operatorname{Ly} \alpha$ transit radius versus wavelength for the fiducial Model 1 to HST STIS data from Ben-Jaffel (2008) and Vidal-Madjar et al. (2008). Points near line center are heavily contaminated by ISM absorption and geocoronal emission and are omitted. Model 1 was designed to agree with the data through adjusting $P_{\mathrm{ss}}$ and $a$ (see Table 4.3). The integrated transit depth, $\delta F / F \approx 10 \%$ (see Table 4.3), is in good agreement with Ben-Jaffel (2008) and Vidal-Madjar et al. (2008).

Figure 4.7 shows $R_{\mathrm{p}}(\nu)$ versus wavelength for models from Table 4.2. The left (right) panel shows the effect of changing $B_{0}(D)$, holding all other parameters fixed. The model number for each line is given in the figure caption, and an additional model with $B_{0}=100 \mathrm{G}$ was included to extend the range of $B_{0}$ well into the strongly magnetized regime. For clarity, Doppler shifts $\Delta \nu=v_{x}\left(\nu-\nu_{0}\right) / c$ due to line of sight motion were ignored in $\sigma_{\nu}$ in the left panel, but are included in the right panel, to 
assess the role of the tidal force in accelerating the fluid. Bulk fluid motion is able to increase the cross section significantly at wavelengths on the steeply falling part of the Doppler core, roughly within $\Delta v= \pm 50 \mathrm{~km} / \mathrm{s}$ of line center.

First consider the effect of the magnetic field in the left panel of Figure 4.7. There is little difference between the $B_{0}=1$ and $10 \mathrm{G}$ models, but in the range $B_{0}=10-100 \mathrm{G}$, the transit depth is observed to grow on the wings of the line. Since bulk fluid velocity effects have been omitted, the increase in transit depth must be due to an increase in hydrogen column over a large area surrounding the planet. Relative to $B_{0}=10 \mathrm{G}$, there is an increase of $50 \%$ for the $B_{0}=50 \mathrm{G}$ model and $250 \%$ for the $B_{0}=100 \mathrm{G}$ model. This result clearly shows that the planetary magnetic field can have an important effect on the transit depth.

In the present study, the base pressure and isothermal temperature are parameters of the model, and the transit depth is most sensitive to these two parameters. The range of these parameters (Table 4.2) was based on the detailed one-dimensional hydrostatic models, including ionization and heating/cooling balance, presented in Trammell et al. (2011). More complete MHD simulations including heating and cooling would determine these quantities self-consistently as part of the solution, and for a known stellar EUV heating rate they would no longer be parameters. In such more complete models, the magnetic field would still be an essentially unconstrained parameter, as it is not measured or constrained by any observation as yet. Figure 4.7 shows that, if $B_{0}$ was the main uncertainty in the model, an upper limit may be placed on magnetic field so that the transit depth is not too large compared to observations. For our fiducial model (1) of HD 209458b, that upper limit would be $B_{0} \gtrsim 10 \mathrm{G}$.

Next consider the effect of changing the rotation rate and tidal force, by changing 
$D$ with all other parameters held fixed. Comparison of the Model 1 lines (solid black line) in the left and right panels shows that Doppler shifts due to bulk velocity in the WZ are small for the fiducial model and the model in which the planet has been moved outward. However, moving the planet inward by $25 \%$ to $D=0.035$ AU has the effect of broadening the wavelength range where $R_{\mathrm{p}}(\nu)$ is large (compare the dashed orange and solid black lines). This is due to bulk fluid velocities Doppler shifting those wavelengths to near line center, where the cross section is large.

Gas which has escaped from the planet is still strongly bound to the star, and may achieve high bulk velocity due to the gravity of the star. In the present case where the tidal force has been axisymmetrized, the effect is symmetric on either side of the star-planet line. In the 3D case, blue-shifted absorption due to gas falling toward the star may achieve even larger velocities. For the chosen box-sizes $r \sim 30 R_{\mathrm{p}}$, the tidal force can accelerate fluid to poloidal velocities $\simeq \Omega r \simeq 60 \mathrm{~km} \mathrm{~s}^{-1}$ in the simulation box. For larger box sizes, even higher velocities may be achieved. However, it is unclear from the present simulations if bulk velocities $\gtrsim 100 \mathrm{~km} \mathrm{~s}^{-1}$ in the flow can affect the line profile, since the steeply falling gas density may not be sufficiently large to give $\tau_{\nu} \gtrsim 1$ at such large distances from the planet. A further uncertainty is the interaction with the stellar wind, which may confine the planetary wind to smaller radii, with smaller acceleration by the tidal force.

To understand the role of magnetic fields on the transit depths, contours of optical depth $\tau_{\nu}(y, z)$ at $200 \mathrm{~km} / \mathrm{s}$ from line center are shown in the y-z plane in Figures 4.8, 4.9 and 4.10. The contours are evenly spaced in $\log \tau$, and white dashed lines show the $\tau=0.1,1$ contours. The area enclosed by the $\tau \gtrsim 1$ contour is optically thick, and contributes significantly to the transit depth. The region between $\tau=0.1-1$ contributes to the transit depth proportional to area $\times \tau_{\nu}$, and so may contribute 
significantly if the increase in area can overcome the decrease in optical depth. For this to occur, the density must not decrease too rapidly outward from the planet. Nearly the entire planetary upper atmosphere is optically thick when observed near line center $(\Delta v=0)$, but moving away from line center the transit depth falls rapidly once the atmosphere becomes optically thin, which occurs at a different value of $\Delta v$ for the range of models shown.

Figure 4.8 is for the hydrostatic initial condition (Equation 4.9) with Model 1 parameters. The contours are smooth ellipses, with equatorial flattening due to the rotation and tidal force. All latitudes make similar contributions to the occulting area.

Figure 4.9 shows the steady-state result for Model $1\left(B_{0}=10 \mathrm{G}\right)$, that results from the initial condition shown in Figure 4.8. Significant differences are readily apparent. A large equatorial DZ with $\tau \sim 0.1-1$ extends out to $r=4 R_{\mathrm{p}}$, while the same contour only extends to $r=2 R_{\mathrm{p}}$ at the poles. The gas in the mid-latitude WZ has significantly smaller optical depth compared to points in the neighboring polar and equatorial DZ's. The left-right asymmetry, most apparent in the $\tau=0.1$ contour, is due to the gas rotation Doppler shift in the cross section. Photons passing through the right side are shifted closer to line center, increasing the cross section, causing the contours to move further from the planet, and vice versa for the left side.

Figure 4.10 shows the steady-state results for the stronger field Model $3\left(B_{0}=\right.$ $50 \mathrm{G}$ ). The $\tau=0.1$ contour surrounding the equatorial DZ now extends to a significantly larger area which is sufficient to overcome the smaller optical depth there. This trend continues to larger $B_{0}$ models, not shown here. Again the mid-latitude WZ and polar DZ have far smaller optical depth compared to the equatorial DZ. Comparing the values of $\dot{M}$ and $\dot{J}$ to $\delta F / F$ in Table 4.3 , the dominant effect is that higher $P_{\text {ss }}$ 
and $a$ lead to both larger $\dot{M}$ and $\dot{J}$, as well as $\delta F / F$. This is due to the higher gas density. The role of magnetic field well into the strongly magnetized regime is also clear, in that larger $B_{0}$ above a critical value leads to larger values of $\delta F / F$ and $\dot{J}$, while also simultaneously smaller $\dot{M}$.

How well did the semi-analytic solutions for $\rho$ and $\boldsymbol{v}$ in Trammell et al. (2011) do at predicting the shape of the magnetospheres and the optical depth contours? Recall that in Trammell et al. (2011) an inner dipole field was fitted to an outer monopole to represent the transition between wind and dead zones. The size of the dead zone was found by stress balance at the equatorial cusp point. Figure 14 of ?) shows the hydrogen column for a sets of models, and can be compared to the optical depth contours in Figures 4.8, 4.9 and 4.10. Model 1 (6) of Trammell et al. (2011) is similar to Model 1 (3) here. The 3-zone structure is evident on both treatments, and many of the trends, e.g. the growth of the equatorial DZ, are evident in both. One key difference is that the equatorial DZ looks more "cuspy" in the numerical calculations where the field is self-consistently calculated, while the semi-analytic calculations give round-shaped dead zones. The dead zone sizes in the two calculations are comparable. The shapes of the wind zones differ between the two calculations due to the different shapes of the field lines, since the poloidal velocity is parallel to the poloidal field. Overall, the semi-analytic methods of Trammell et al. (2011) give rough quantitative agreement with the simulations here, at least near the planet where the assumed field shape of Trammell et al. (2011) is approximately correct. Further from the planet, the backward bending of field lines, and the deviations from corotation become large, and are not taken into account in the analytic models in Chapter 2. 


\subsubsection{Results for HD 189733b}

In this section the exercise from section 4.3.2 is repeated. Figure 4.11 compares the transit depth for the fiducial (Model 1) model for HD 209458b to three models for HD 189733b, a fiducial model similar to Model 1 but with the adjusted planetary and orbital parameters for HD $189733 \mathrm{~b}\left(R_{p}=1.14 R_{\mathrm{Jup}}, M_{\text {star }}=0.806 M_{\text {solar }}, D=\right.$ 0.031 AU) a model with base pressure $P$ increased by $400 \%$, and a model with $40 \%$ higher sound speed $\left(14 \mathrm{~km} \mathrm{~s}^{-1}\right)$. Due to the higher ratio of $M_{\mathrm{p}} / R_{\mathrm{p}}$ for HD $189733 \mathrm{~b}$, the scale height is smaller, which leads to a smaller amount of Ly $\alpha$ absorption. In the model spectra, this is manifested by small transit depth on the wings, as well as a smaller central Doppler core region. The integrated transit depth of the fiducial model is $\simeq 4 \%$, while the transit depth for the ' $4 P$ ' model in Figure 4.11 is $\simeq 5 \%$,

which is in agreement with the observations by Ehrenreich \& Désert (2011). The motivation behind the ' $4 P$ ' model is that the significantly closer orbital distance of HD 189733 b to its already more magnetically active host star results in a higher level of incoming EUV flux incident on the upper atmosphere, setting the $\tau \sim 1$ layer at a lower altitude and therefore higher local gas density.

\subsection{Summary}

The MHD wind model simulations presented in this Chapter demonstrate that, for an extended range of latitudes, the planet's magnetic field can restrict a thermally-driven wind. In Trammell et al. (2011), dipole field geometry and expected field strengths of hot Jupiters were used to estimate the size of the closed field line regions, where an outflow is quenched by rigid magnetic field lines. The inclusion of the stellar tide was also shown to quench the outflow in the polar region due to the higher potential 
barrier. The estimated equatorial dead-zone sizes were $\sim 3-10 R_{\mathrm{p}}$ for the parameters of interest.

MHD simulations permitted the relaxation of the assumption of a steady-state field geometry, as hydrodynamic stresses and magnetic stresses in the thermallydriven outflow from the hot inner boundary were computed self-consistently through force balance both across and along field lines. The simulation results verify the main features of the semi-analytic models of Trammell et al. (2011) in the structure of the upper atmosphere through their dependence on $B_{0}$ (Figures 4.1 and 4.2), and stellar tide strength (Figures 4.3 and 4.4). The MHD wind model also permitted a self-consistent calculation of the mass and angular momentum loss rates, which were presented in Section 3.3 as a function of stellar tide strength, and again in Section 4 as a function of the integrated Lyman $\alpha$ transit depth, $\delta F / F$ (the observable quantity). Our results are most consistent with a pressure of 50-60 nbar and a temperature of $\sim 10^{4} \mathrm{~K}$ at the base of the thermosphere for HD 209458b, corresponding to a mass loss rate $\dot{M} \simeq 3 \times 10^{11} \mathrm{~g} / \mathrm{s}$ and an angular momentum loss rate $\dot{J} \simeq 6 \times 10^{28} \mathrm{~g} \mathrm{~cm}^{2} / \mathrm{s}$.

A central result of this thesis is that for sufficiently large magnetic field, the large resulting equatorial DZ may dominate the optically thick area which gives rise to the transit depth signal. In this strongly magnetized regime, we find the transit depth increase with the magnetic field. In a future study in which the heating and cooling of the atmosphere are determined self-consistently, and not given as parameters, this strong dependence on the magnetic field may allow an upper limit to be placed on the planet's magnetic field so as not to over-predict the transit depth.

A key result of Trammell et al. (2011) which we have confirmed is that the optically thick region around the planet is dominated by the equatorial DZ, a region that is bound to the planet, even if it is partially outside the Hill (Roche-lobe) radius. 
Hence the fact that Ly $\alpha$ absorption occurs out to near the Roche-lobe radius does not directly imply the absorbing gas is escaping (e.g. Vidal-Madjar et al. 2003). The MHD model does exhibit gas in the mid-latitude WZ which is escaping, however, the Ly $\alpha$ transmission spectrum is less sensitive to the gas in this region as the optical depths are lower (see Figure 4.10).

The observational uncertainties in Figure 4.7 cannot precisely constrain the pressure at the base of the warm $\mathrm{H}$ layer, the integrated transit depths computed from the model Lyman $\alpha$ spectra presented in Section 4 provided another quantitative comparison with observations. The high sensitivity of the integrated transit depth on the pressure at the base of the warm $\mathrm{H}$ layer suggests that this observable quantity can probe and constrain the conditions in the thermosphere of highly irradiated hot Jupiters. At the same time, the numerical models presented here provide complementary information about the resulting expected mass and angular momentum loss rates, which are inaccessible by observations.

\subsection{Appendix: Shear Layer and Current Sheet Near the Equatorial Dead-Zone/Wind-Zone Bound- $\operatorname{ary}$}

At the boundary between the equatorial DZ and mid-latitude WZ, there are sudden changes in fluid velocity and magnetic field over short distance. The origin of this shear layer and current sheet was discussed in Section 4.2.1. As the simulations presented in this Chapter do no include explicit viscous forces and Ohmic diffusion, it is the numerical effects contained with the ZEUS-MP code that control the behavior of the solutions at these discontinuities. 
The possible effects from numerical diffusion came to our attention due to the spurious bump in density at the equator shown in Figure 4.5, where a rise in density occurs inside the Hill radius. This behavior contradicts basic analytic considerations. It was shown in the Appendix of Trammell et al. (2011) that, in steady state, the Bernoulli constant

$$
W \equiv \frac{1}{2} v^{2}+a^{2} \ln \rho+U_{\text {rot }}
$$

(where $v$ and $U_{\text {rot }}$ are defined in a frame corotating with the planet) must be a constant along a given field line in the dead-zone (see Equation A10 of Trammell et al. (2011)). Since $v=0$, our choice of base density, Equation 4.9, indicates that $W$ should be a constant throughout the dead-zone. The force-balance equation A8 of Trammell et al. (2011) implies immediately that the Lorentz force must vanish in the dead-zone. If this is the case, the density along the equator can only increase with distance outside the Hill radius. This contradicts the numerical results in Figure 4.5.

The spurious density bump seems to be due to numerical effects near the deadzone/wind-zone boundary, although the origin is rather subtle. In hindsight, it is not surprising that the boundary would be difficult to treat numerically, because there are discontinuities in all quantities: density and each of the three components of the velocity and magnetic field. The discontinuity in magnetic field, in particular, is difficult to treat accurately (see, e.g., Fig. 16 of Stone \& Norman 1992b). One way to increase the accuracy is to increase the spatial resolution, which we have done for the fiducial Model 1. In Fig. 4.12, we show the equatorial density profile at four different resolutions $(100 \times 100,272 \times 200,400 \times 400$, and $800 \times 800)$, normalized by the substellar point density. It is clear that the spurious density enhancement decreases with increasing resolution, although even at $800 \times 800$ it does not disappear 
completely.

This density enhancement is caused by magnetic forces (see Fig. 4.13), which should vanish throughout the dead-zone according to the analytic considerations mentioned earlier. At the heart of these considerations is the constancy of the Bernoulli constant $W$ throughout the dead-zone. It breaks down in the numerical simulations, as illustrated in Figure 4.14, where we show the distribution of the Bernoulli constant and poloidal velocity for a selected region for the standard resolution $272 \times 200$. Note that $W$ is indeed very close to the expected value over most of the dead-zone (where the poloidal velocity is small, see the right panel), except in a layer near the DZ/WZ boundary, where deviation of order $1 \%$ is evident; this is also the region where the magnetic forces become appreciable, and the density starts to increase outward spuriously. As the resolution increases, the boundary layer shrinks in size. This should serve as a cautionary tale for future simulations of hot Jupiter magnetospheres, especially in 3D, where the resolution will necessarily be coarser than in 2D. Nevertheless, the basic three-zone structure of the magnetosphere is robust.

Although the density profile in the equatorial DZ is resolution dependent, the transit depth varies for the four models shown in Figure 4.12 vary only slightly, with values $\delta F / F=0.0943,0.1002,0.1026,0.1031$ for resolutions $100 \times 100,272 \times 200$ (Model 1), 400x400 and 800x800. This variation with resolution is far less than the variation from changing parameters in Models 1-9. This lends confidence that the broad conclusions are not affected much by finite numerical resolution effects. 


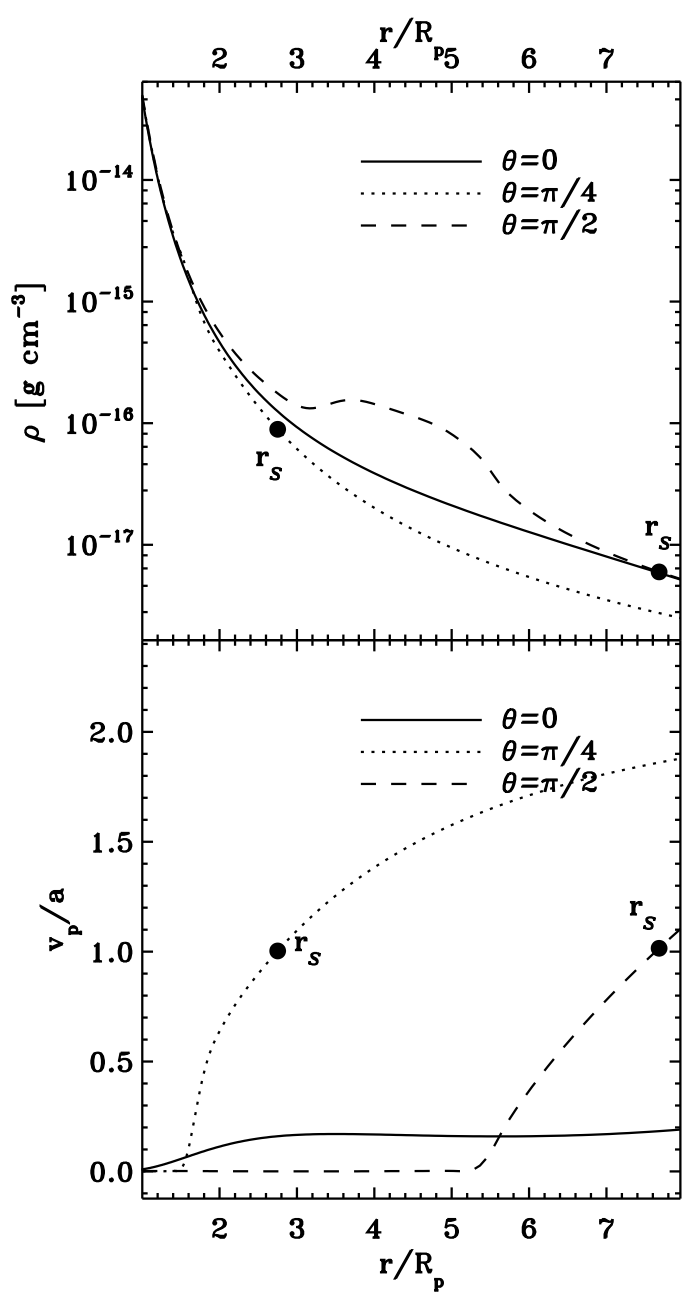

Fig. 4.5.- 1D radial profiles of density $\rho(r)($ top $)$ and poloidal velocity $v_{p}(r)$ (bottom) in units of the sound speed $a$ for the fiducial Model 1 at three representative angles. The three angles shown in each panel are $\theta \approx 0$ (solid black line), $\theta \approx \pi / 4$ (dotted black line), and $\theta \approx \pi / 2$ (dashed black line). Sonic point locations are indicated by the black symbols. 


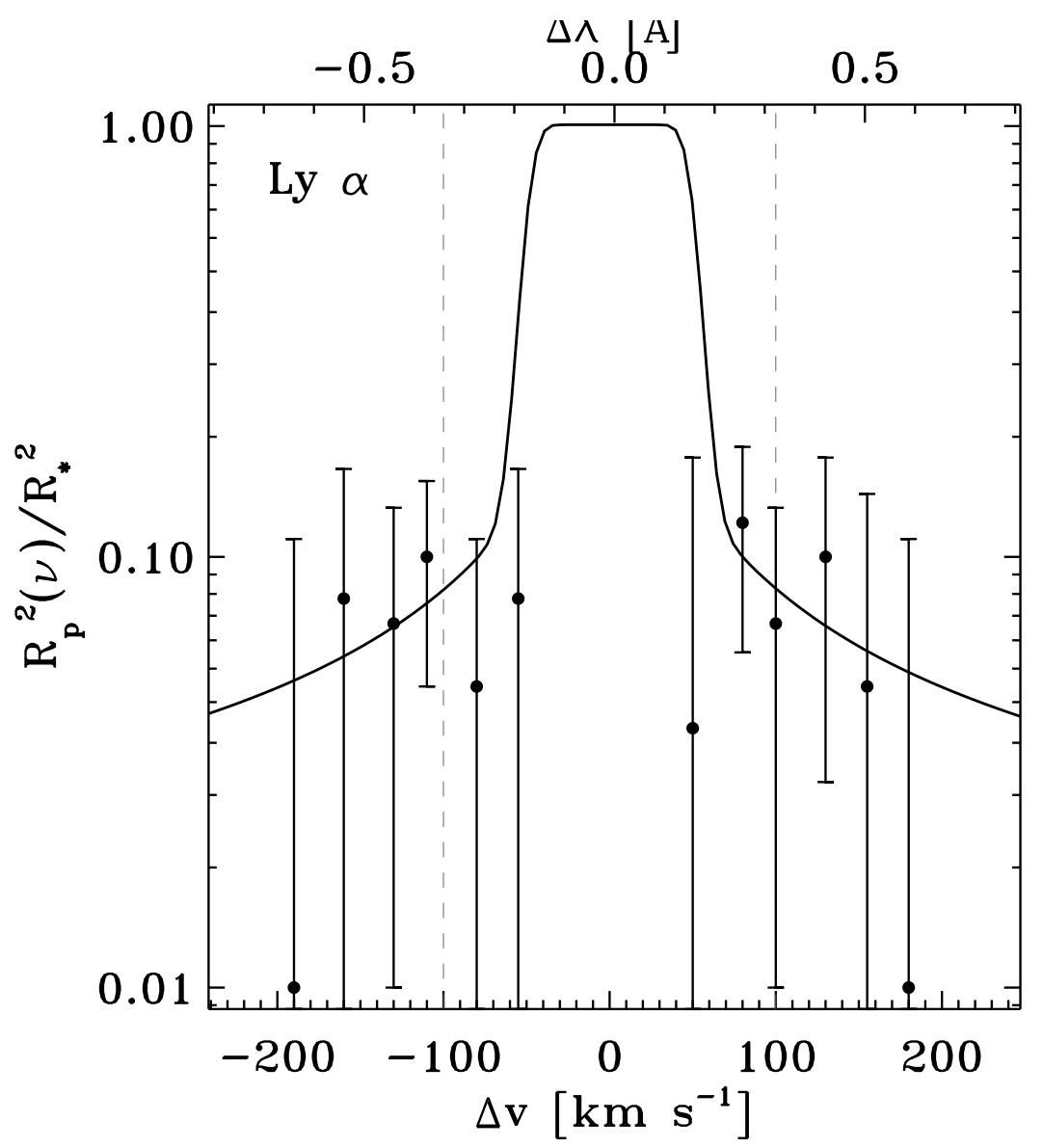

Fig. 4.6.- Model 1 transit curve for comparison to the transit depths for HD 209458b from Ben-Jaffel (2008). 

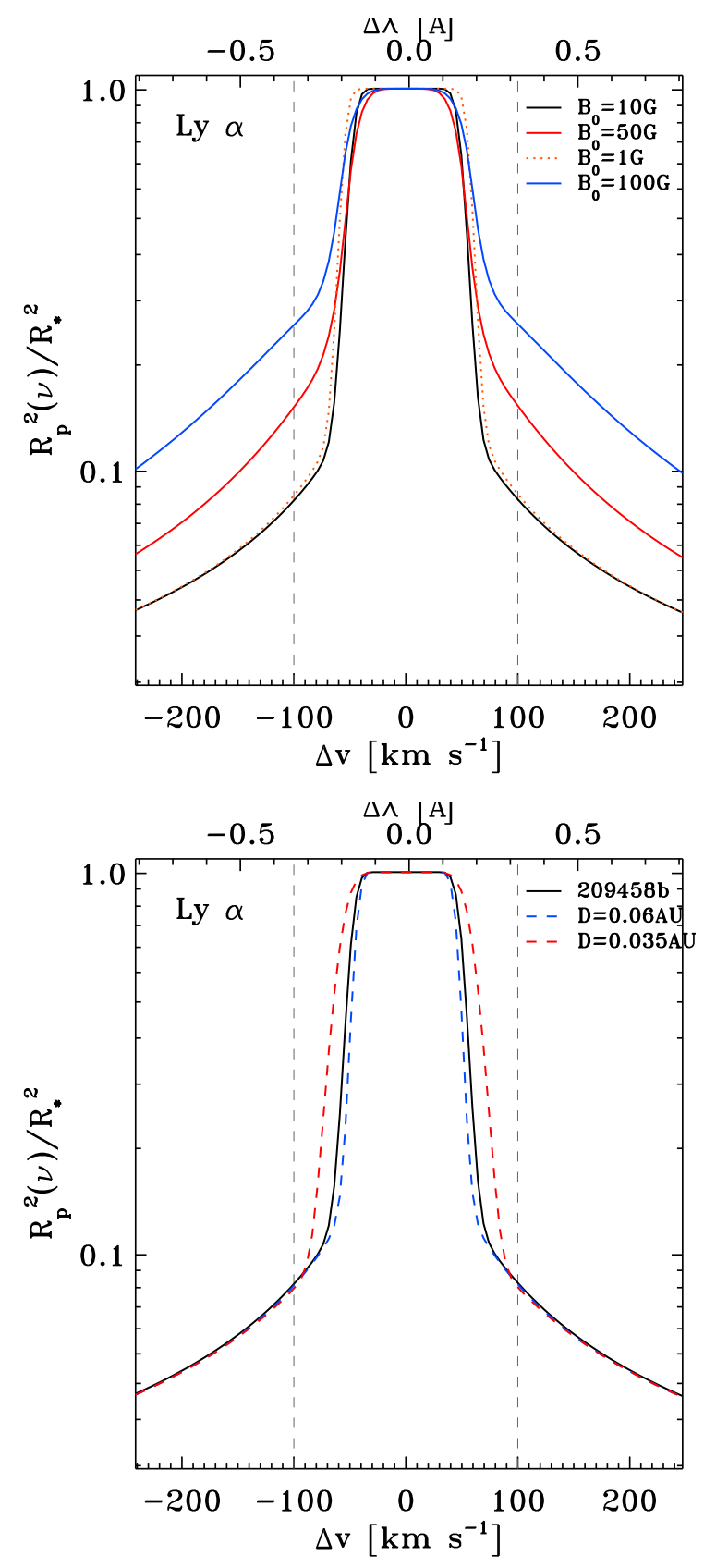

Fig. 4.7.- Ly $\alpha$ transit depth (Equation 4.25) versus wavelength in velocity units for select models. The left panel compares models with different $B_{0}$ (Model 1, $B_{0}=10 \mathrm{G}$, solid black line; Model 2, $B_{0}=1 \mathrm{G}$, dotted orange line; Model $3, B_{0}=50 \mathrm{G}$, solid red line; a $B_{0}=100 \mathrm{G}$ model, solid blue line), while the right panel compares models with different $D$ (Model 1, $D=0.047$ AU, solid black line; Model 8, $D=0.035$ AU, dashed red line; Model $9, D=0.06 \mathrm{AU}$, dashed blue line). In each panel, only the one parameter is changed, with all others held fixed at Model 1 values. For clarity, bulk Doppler shifts are ignored in the left panel; they only affect the line profile at $\Delta \boldsymbol{v} \lesssim 50 \mathrm{~km} \mathrm{~s}^{-1}$ from line center. Bulk Doppler shifts are included in the right panel, as the tidal force may give rise to additional acceleration. Vertical lines are placed at $\Delta \boldsymbol{v}= \pm 100 \mathrm{~km} \mathrm{~s}^{-1}$ for convenience. 


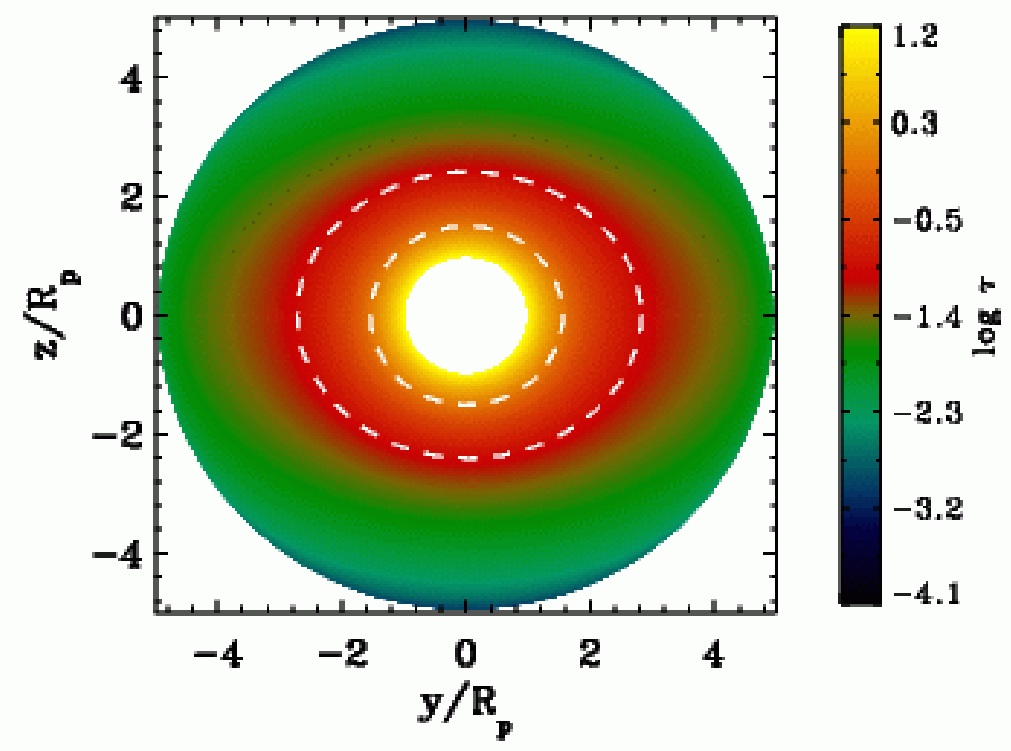

Fig. 4.8. - Contours of Ly $\alpha$ optical depth $\tau(y, z)$ at $\Delta v=200 \mathrm{~km} / \mathrm{s}$ from line center for the hydrostatic initial condition with Model 1 parameters. The inner (outer) dashed white line shows $\tau=1(0.1)$.

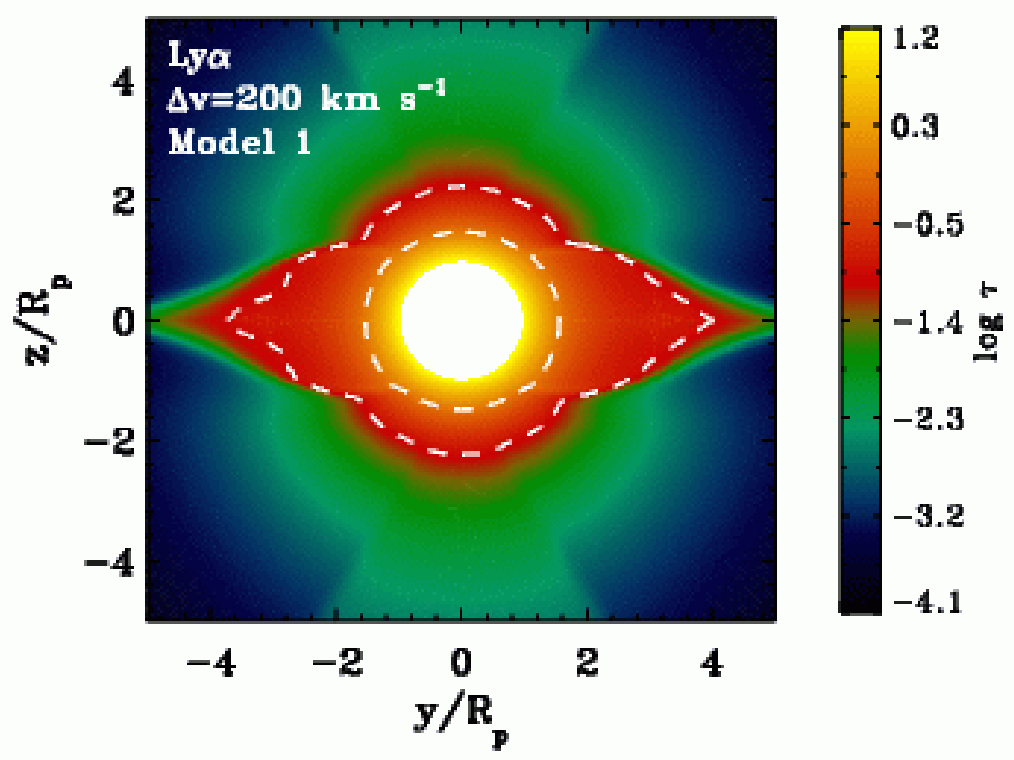

Fig. 4.9.- Same as Figure 4.8 for the Model 1 simulation. 


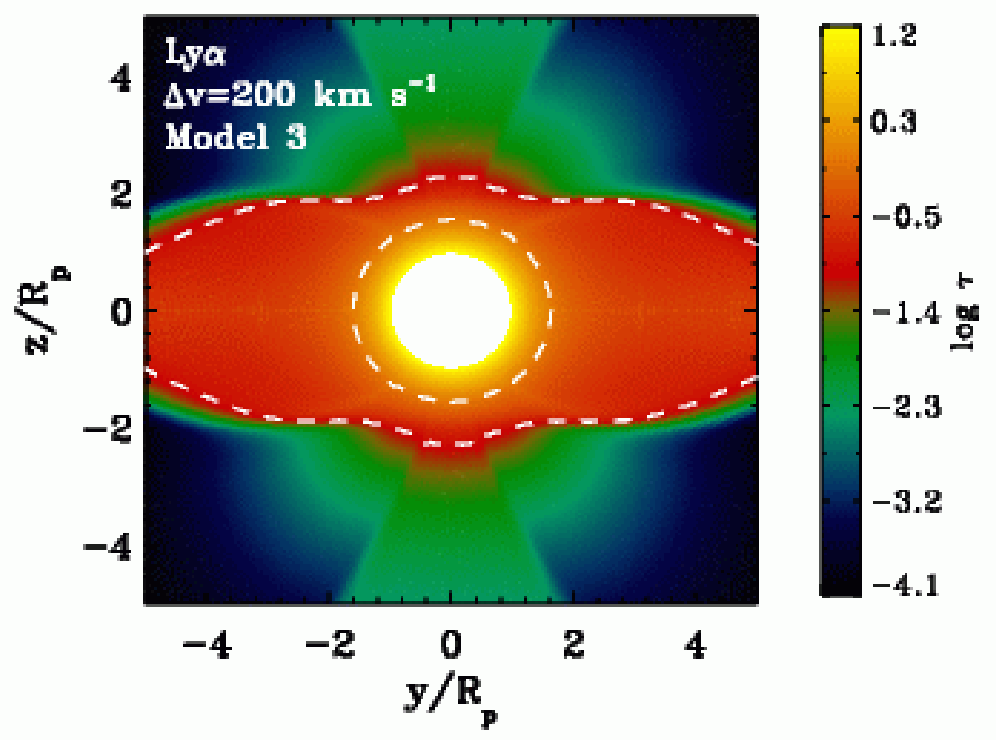

Fig. 4.10.- Same as Figure 4.9 for the Model 3. 


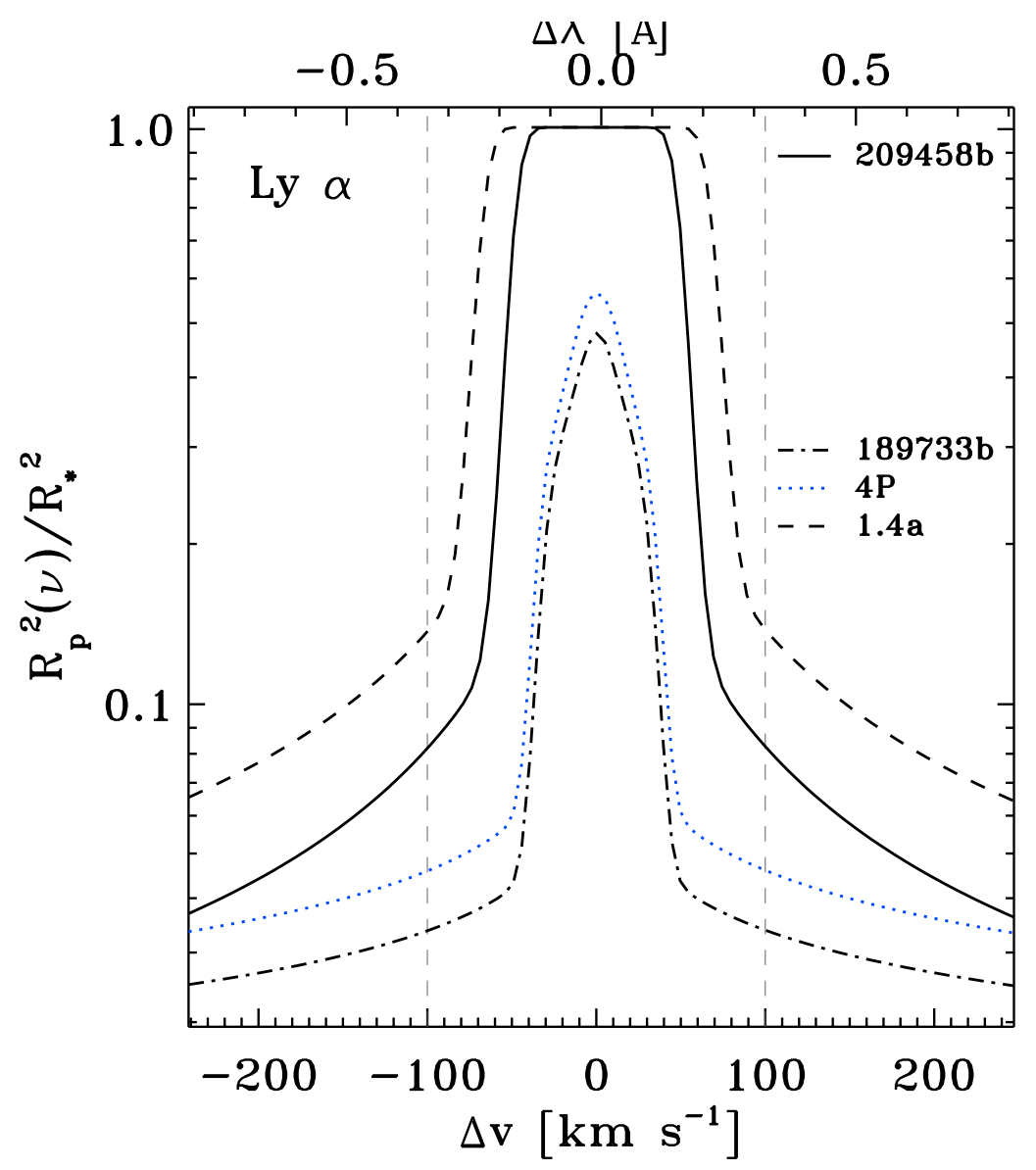

Fig. 4.11. - The fiducial model for HD 209458b in comparison to three models for HD 189733b. The dot-dashed line is the frequency-dependent transit depth for parameters matched to HD 189733b, while the blue-dotted and dashed lines vary the base density and sound speed, respectively. 


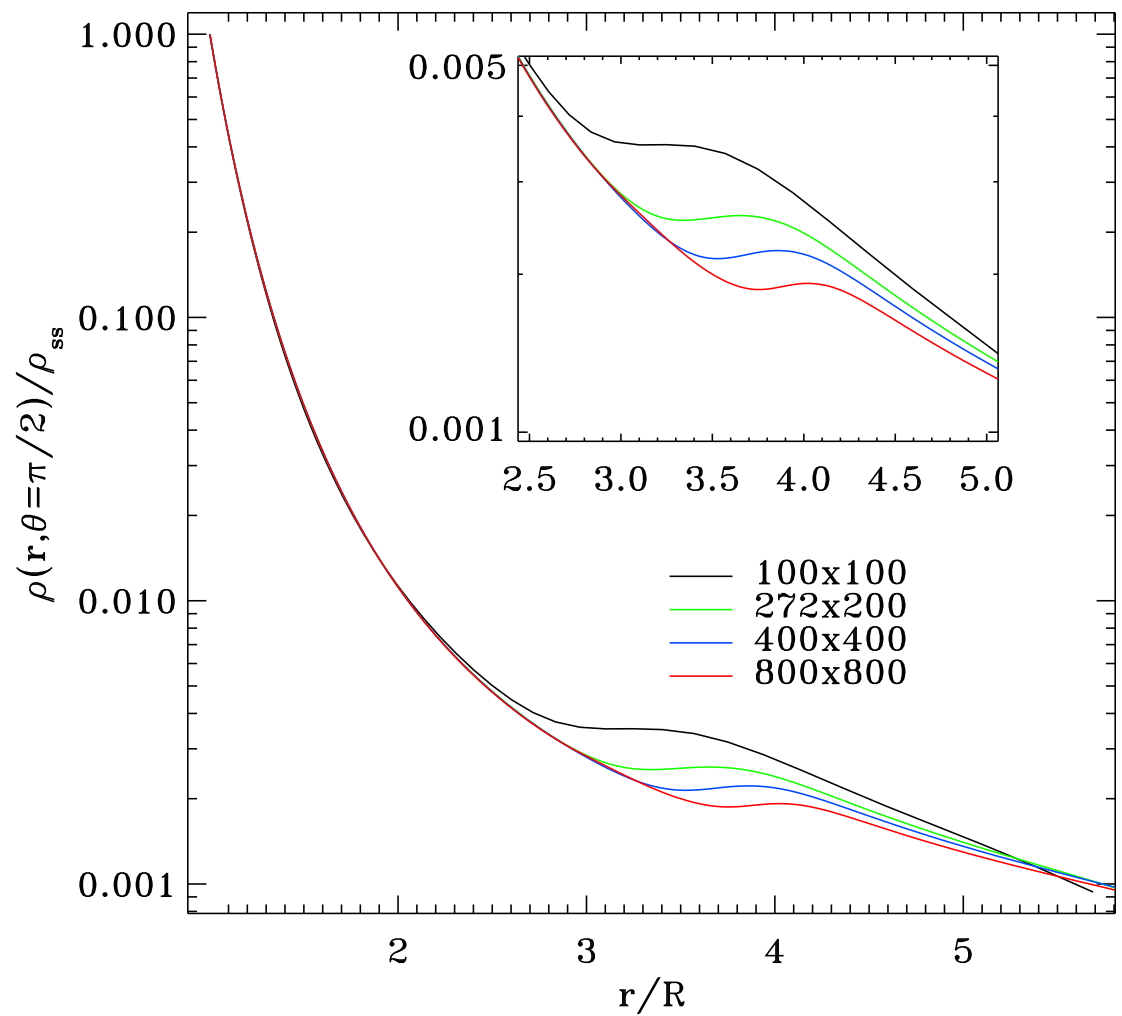

Fig. 4.12.- The equatorial density profile for Model 1 at four different grid resolutions in steady-state (fiducial resolution is 272x200). 


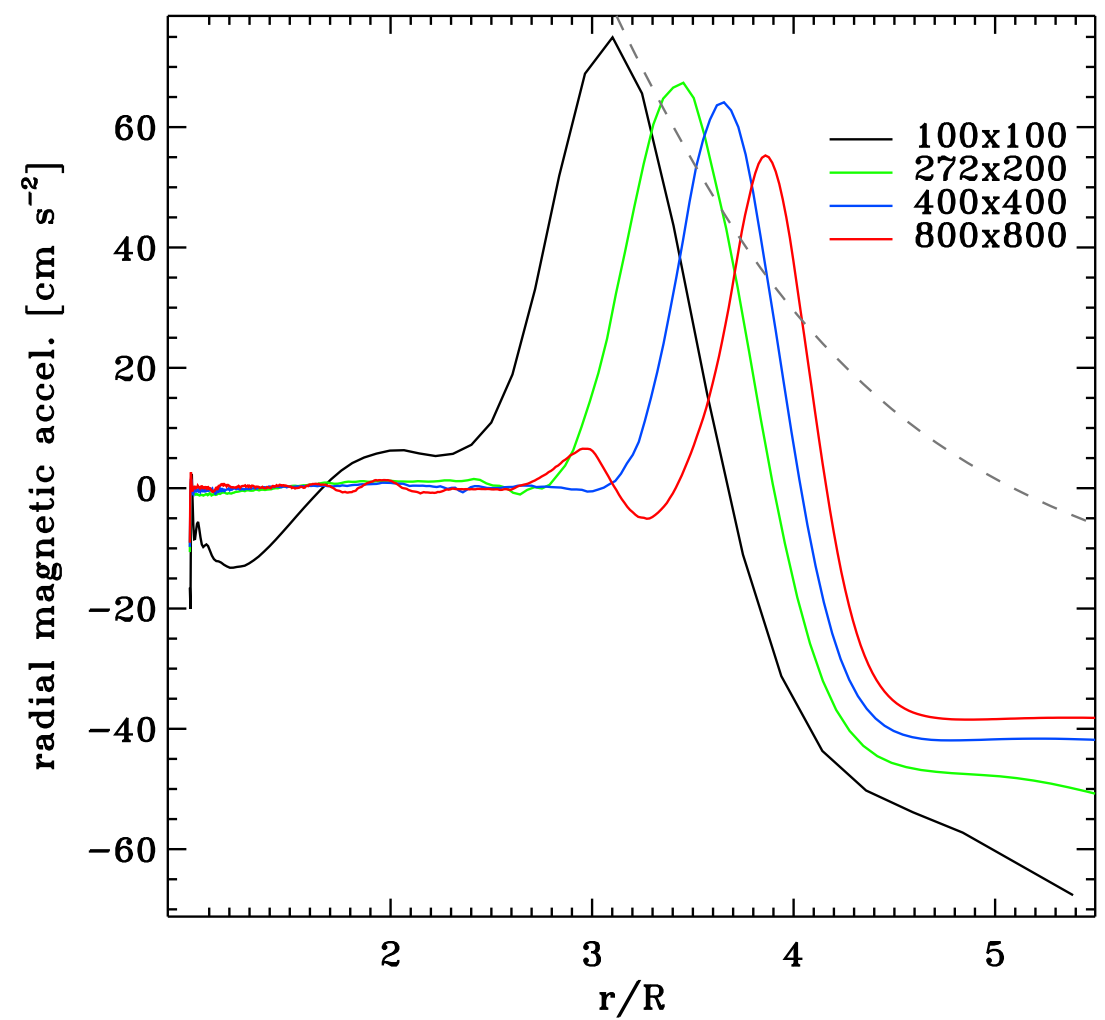

Fig. 4.13. - The radial magnetic forces for Model 1 at three different grid resolutions in steady-state. The gray dashed line represents the effective gravity. 


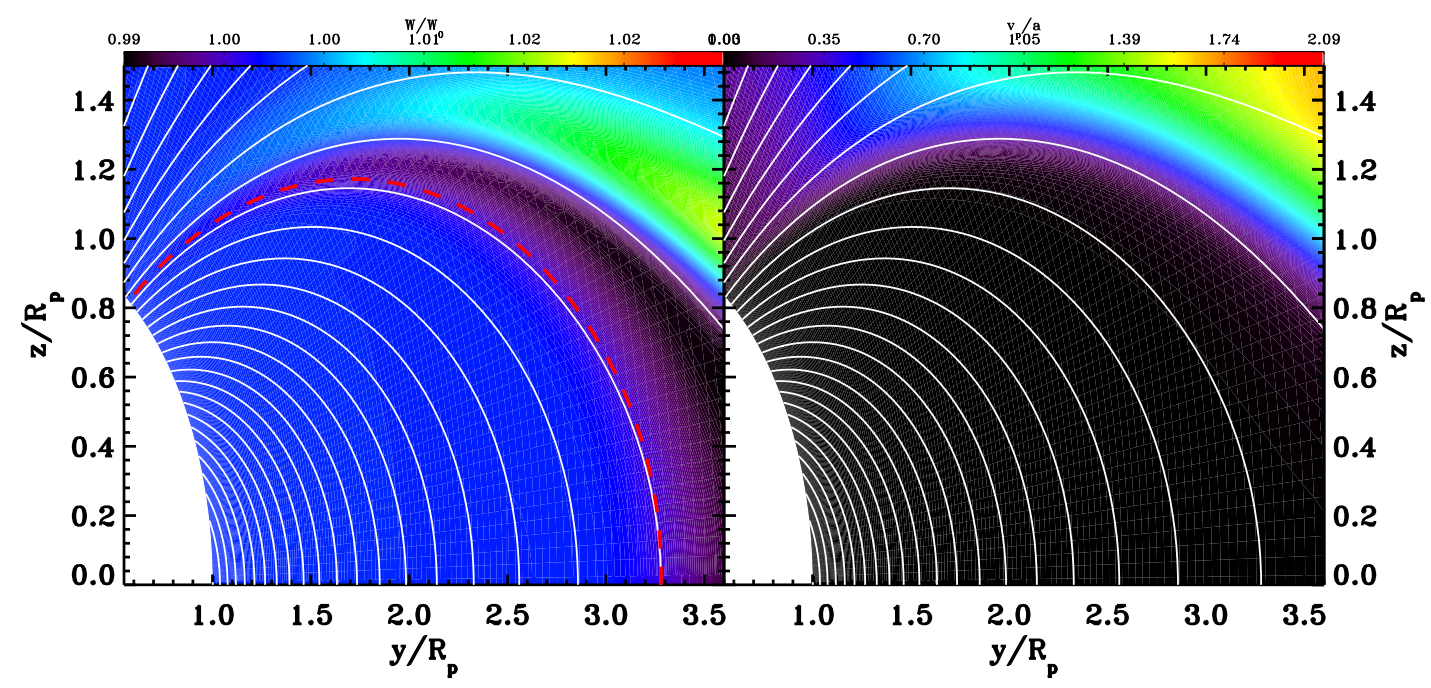

Fig. 4.14. - The variation of the Bernoulli constant relative to the base value for a region including the DZ/WZ boundary layer, and the poloidal velocity distribution for the same region. The traced field line is highlighted in red. 


\section{Chapter 5}

\section{Summary \& Future Work}

In Chapter 1, I motivated the expected upper atmospheric structure for hot Jupiters such as HD 209458b and HD 189733b. Features of the model include large gas scale heights, and possible atmospheric escape, from an extended upper atmosphere around the planet that has been probed by transmission spectroscopy using strong atomic resonance lines. I have also summarized the variety of observations that establish the existence of an extended upper atmosphere.

Chapter 2 set the stage for a numerical model using the machinery of the ZEUSMP code that is summarized in Chapter 3. The numerical model considers the scenario in which the transit depth is due to a layer heated due to the photoionization of $\mathrm{H}$ in the planet's atmosphere. Through detailed magnetohydrodynamic (MHD) simulations that relax several of the assumptions necessary for the analytic models in Chapter 2, the simulations expand on the work of Trammell et al. (2011), where the magnetic effects are considered semi-analytically. The goal of numerical simulations is to confirm the validity of the analytically-obtained prediction of a three-zone structure.

In the following sections, I summarize the content of the previous chapters, and 
I also outline future directions that builds upon the models that I have presented in this thesis.

\subsection{Analytic MHD Models}

Trammell et al. (2011) showed that the addition of the planetary magnetic field leads to the formation of an equatorial "dead-zone" (DZ) - a static region where the wind ram pressure is insufficient to overwhelm magnetic stresses and open the field lines into an outflow. This effect is well known in the classical MHD stellar wind theory (e.g., Mestel 1968). Paper I found a second static region near the poles where the wind can be shut off by the increased gravitational potential barrier from the stellar tide. In the strong tide limit, a wind-zone is then expected to exist only at intermediate latitudes.

A thermal model was constructed under the assumption of a balance between photoelectric heating and collisionally-excited Lyman $\alpha$ cooling. Short mean free paths $\left(\sim 10 \mathrm{~km}\right.$ for $\left.T=10^{4} \mathrm{~K}\right)$ and low drift velocity for the electron-proton-H gas using the relevant timescales and collision rates in the upper atmosphere, motivated a single fluid approach to compute the analytic column density structure surrounding the planet that captures physical properties of the planet and tidal gravity from the star. These results show that for certain ratios of escape speed to gas sound speed, and rotation speed to sound speed, my model calculations can produce a sufficient column at several planetary radii to be consistent with the transit observations of HD 209458b (e.g., Vidal-Madjar et al. 2008).

A limitation of the analytic models in Chapter 2 is that they ignore magnetic forces, which means that the poloidal magnetic geometry was assumed rather than computed self-consistently. Another limitation is that the fluid was assumed to be 
corotating with the planet everywhere, including the wind zone, where the corotation is expected to break down at large distances. In Chapter 4, I overcame these limitations by performing MHD simulations, which compute the magnetic field and fluid rotation self-consistently. This allowed for the calculation of the mass and angular momentum loss rates, as well as the density and velocity profiles required to compute transmission spectra, which I summarize in the next section.

\subsection{MHD Simulation Results}

The simulations confirm the three-zone structure - polar dead zone, mid-latitude wind zone, and equatorial dead zone - predicted in previous analytic calculations in Trammell et al. (2011). For a magnetic field comparable to that of Jupiter, the equatorial dead zone, which is confined by the magnetic field and corotates with the planet, dominates the transit signal; the gas escaping in the mid-latitude wind zone is found to have a smaller contribution to the transit depth than the equatorial dead zone. Transmission spectra were compared to the available HST STIS and HST ACS data for HD 209458b and HD 189733b, demonstrating that a range of model parameters can make the models consistent with the data. A key result was that the transit depth increases strongly with magnetic field strength when the hydrogen ionization layer is magnetically dominated, for dipole magnetic field $B_{0} \gtrsim 10 \mathrm{G}$.

The main implication for observations is that the observed Lyman $\alpha$ transit depth is sensitive to magnetic field strength, in addition to standard quantities like the ratio of thermal to gravitational binding energies. Therefore, an upper atmospheric transit signal corresponding to an area in excess of the Roche Lobe is not the correct criteria to apply to results from transmission spectroscopy that have suggested the data provide evidence of mass loss in a hydrodynamic wind. The presented MHD 
model demonstrates that the key ingredients for a more complete physical model include accounting for magnetic forces in the upper atmosphere. Due to the variety of models and the sensitivity of the transit depth to $B_{0}$, this suggests a fundamentally new way to learn about the magnetic field strengths in hot Jupiters, which have so far been unreachable by direct observations.

\subsection{Extension to 3D-MHD Simulations}

An extension of the wind model to fully 3D calculations using ZEUS-MP and the accomplished boundary conditions would generalize the results of this thesis to more complex magnetic geometries. There are several key advantages to moving toward 3D-simulations. In order to capture both the tidal and centrifugal terms in the effective gravitational potential, I have been necessarily restricted to a coordinate system centered on the planet in the corotating frame. 3D runs would permit me to relax the assumption of corotation and tidal synchronization for the planet's spin. A second advantage is that I could implement a numerical prescription for time-dependent energy input from the star on the dayside, and physical grid-zones in the $\phi$-direction would permit me to see how the resulting pressure gradients advect fluid as functions of latitude and longitude. This has the highest significance for accurate calculations of the conditions at the base of the thermosphere, where significant photoionization is taking place. Variations in the base density would affect the background density of the upper atmosphere, and combined with the available acceleration from thermal pressure gradients, and would yield full 3D information about mass/angular momentum loss from the planet.

Non-ideal effects can strongly influence the coupling of gas to the magnetic field as well as the heating rates in atmospheres. It is beyond the scope of my thesis to 
be able to implement non-ideal MHD physics into my simulations, and I defer this to the possibility of estimating the importance of non-ideal effects in the atmosphere, as is currently being done already in the literature (e.g., Perna et al. 2010; Batygin \& Stevenson 2010). However, I am focused specifically on how including the planet's magnetic field and accounting for the stellar tide fundamentally influences the overall structure and mass-loss rates for upper atmospheres. Detailed calculations of Ohmic heating and dissipation in the atmosphere would have a high degree of applicability at higher pressures, deeper in toward the planet's interior, and recent theoretical work has shown this could be most important to circulation patterns deep in the atmosphere, far below the altitudes that are the subject of my proposed 3D simulations.

\subsection{Additional 3D-Model Applications}

This section outlines two possible extensions to the successful development of 3DMHD simulations in ZEUS-MP, which would be further pursued if the initial 3Dmodels of hot Jupiter upper atmospheres is successful.I now give two examples of possible scientific return from expanded models that would exploit the 3D capabilities of ZEUS-MP.

\subsubsection{The Stellar Wind Contribution}

As one extension for the proposed 3D simulations centered on the planet, one might hope to somehow account for the importance of the incoming magnetized stellar wind entering the simulation box through the construction of a self-consistent boundary condition. Magnetic interactions between these planets and their host stars motivate, at a minimum, a qualitative understanding of the effects on the incoming stellar wind on our overall pictures of planetary outflows in these extreme environments. 
An extrasolar giant planet with an internal dipole magnetic field orbiting in a magnetized stellar wind results in a field shape that is dynamic, and is a direct result of the change in the direction of the stellar field as seen by the planet as it orbits its star. Large amounts of magnetic flux are transported from the day to night sides every orbit, which is analogous to the changes seen in the Earth's magnetosphere during transitions from north to south oriented interplanetary field. This flux transport will lead to significant ionospheric heating from processes such as ion-neutral drag (e.g., Schunk \& Nagy 2004). The physics of magnetically-driven outflows, coupled with better estimates of heating and cooling in the upper atmosphere, can therefore be extended to help explain the magnetic coupling of hot Jupiters with their parent stars. The structure of the magnetospheres of EPs can modify mass and angular momentum loss significantly, which are quantities that control the future evolution of the planet.

\subsubsection{Perceived Impact}

Mass/angular momentum loss rates can also be calculated for a wider range of exoplanet atmospheres and orbital parameters. The addition of a model for the planetstellar wind interaction can also lead to estimates of the evolution and survivability of planetary atmospheres.

A modification of the momentum and energy equations can also be made to track additional particle species to construct additional column density maps and to characterize the expected structure of magnetospheres for a wide range of systems with more complex upper atmospheric compositions. Synthetic spectra for Lyman $\alpha$ as well as for other resonance lines in $\mathrm{Na}, \mathrm{O}$, and $\mathrm{C}$ can be compared with observational results of existing and upcoming NUV/optical/NIR spectroscopy of additional tran- 
siting planets. The models can also be enhanced to include the wake of the orbiting planet as its magnetosphere interacts with the surrounding magnetized stellar wind. The stellar wind parameters of interest can come from observational and theoretical constraints on properties of exoplanet host stars.

The simulation results can also make contact with models of the lower atmosphere for HJs to add to a more continuous model for the overall structure of their atmospheres, perhaps even leading to an improvement in setting base conditions for the upper atmosphere models.

For $\mathrm{H}$ photoionization with cross-section $\sigma_{H} \simeq 10^{-18} \mathrm{~cm}^{-2}$, proton mass $m_{p}$ and surface gravity $g$, the ionization layer occurs at pressure $P \simeq g m_{p} / \sigma_{H}$, or $\simeq$ $10^{-3}$ dyne $\mathrm{cm}^{-2}\left(\mathrm{~g} / 10^{3} \mathrm{~cm} \mathrm{~s}^{-2}\right)$ for a gravity similar to Jupiter. Therefore $P_{\text {gas }} \sim 10^{-9}$ bar, which naturally complements models that include lower components of the atmosphere - the deep regions in thermal equilibrium, the transport-dominated regions at intermediate pressures, and the lowest pressure regimes below the thermosphere where photochemical processes dominate. Figure 5.1 is taken from Figure 1 of Line et al. (2011), which shows estimated temperature-pressure profiles for the hot Neptune GJ436b. Given the pressure regime for the thermobase, this lies at the upper boundary of Figure 5.1. Figure 5.2 shows example abundances taken from a solar abundance model of (Line et al. 2011), which shows a region at the lowest pressures where $\mathrm{H}$ begins to dominate the composition.

Given the above estimates for the pressure where photoionization takes place, magnetic pressure $P_{\text {mag }}=B^{2} / 8 \pi \sim 10^{-6}$ bar for $B=B_{\text {Jupiter }}$, which exceeds the gas pressure by a factor of $\sim 10^{3}$ ! This illustrates why the planet's magnetic field dominates the dynamics, and shows why an MHD wind model for photoionization-driven outflows from a magnetized planet can use the upper boundary of lower atmosphere 
models as base conditions for the bottom of a thermosphere. The addition of a model for the upper atmosphere then provides for a more complet complete picture of exoplanetary atmospheres that subtends the full range of pressures and dominant physical processes of interest.

A description of the upper atmosphere complements photochemical and global circulation models that predict conditions in the lower atmosphere. Thermochemically derived mixing ratios and T-P profiles, set the base of a thermosphere. Combining these calculations therefore significantly advances exoplanet science by providing a complete atmosphere model that couples chemistry in the lower atmosphere with evolutionary consequences of dynamics in the upper atmosphere. The results will help guide further exoplanet characterization. 


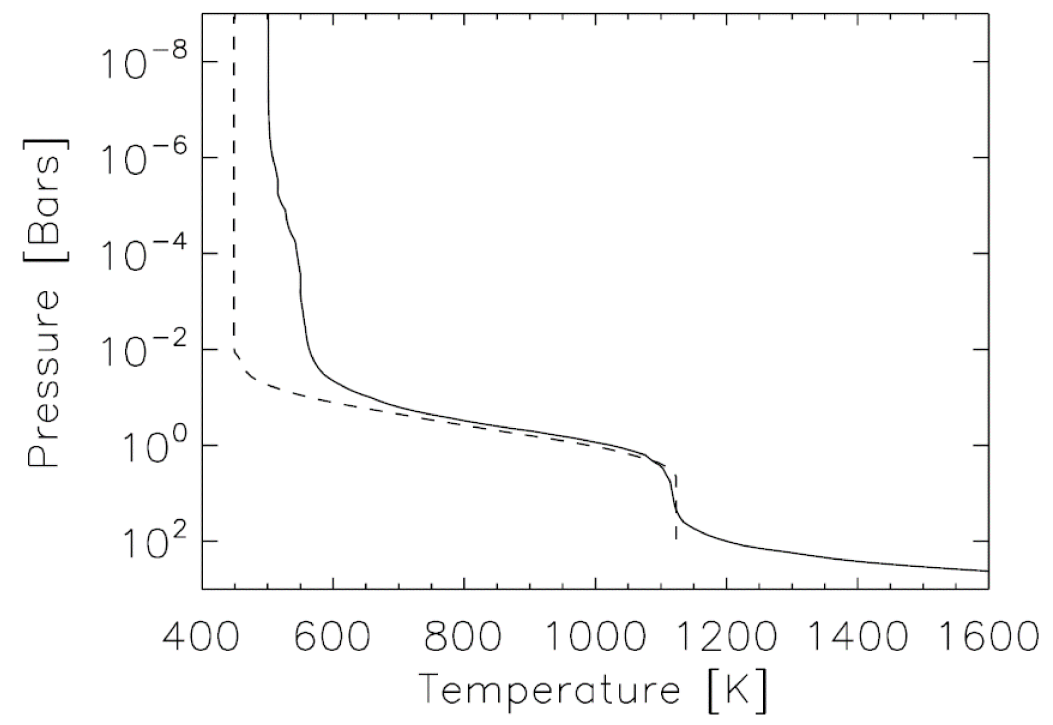

Fig. 5.1.- Estimated T-P profiles for GJ436b taken from Figure 1 of (Line et al. 2011). A pure $\mathrm{H}$ upper atmosphere would become optically thick to Lyman $\alpha$ photons near a pressure $\sim 10^{-9}$ bar, but magnetic forces for a Jupiter-strength field would begin to dominate already at a pressure $\sim 10^{-6}$ bar. 


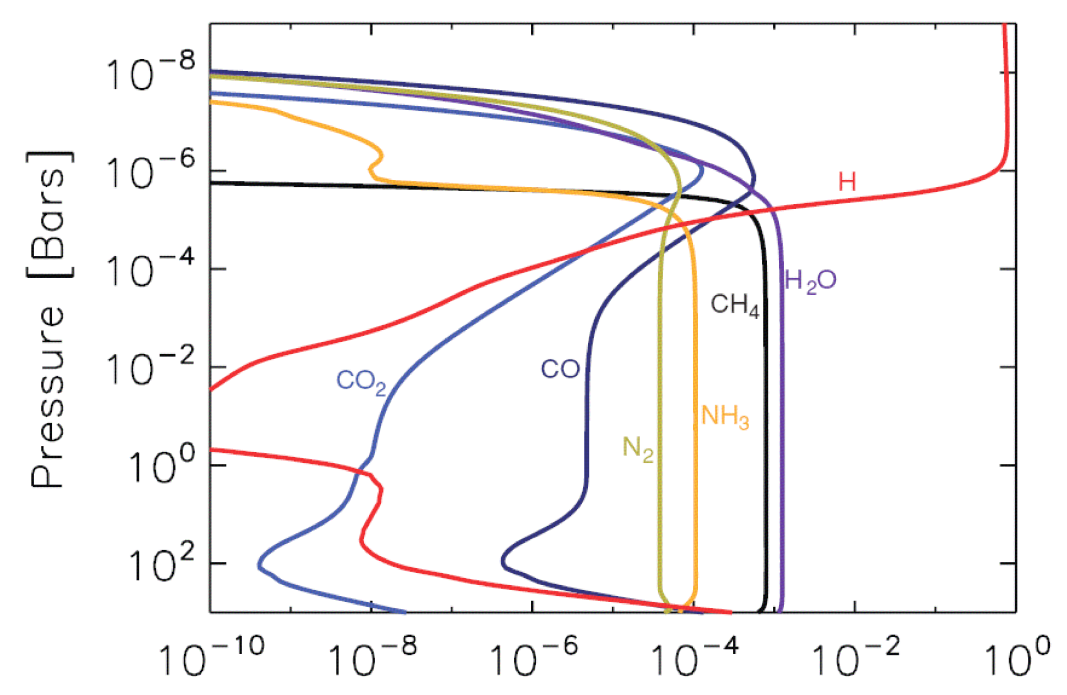

Fig. 5.2.- Atmospheric pressure vs. mixing ratio for solar metallicity, taken from Figure 6 of (Line et al. 2011). This example for the hot Neptune GJ436b shows how the atmospheric composition simplifies greatly as one approaches pressures at the thermobase. These models of the lower atmosphere provide base conditions for an MHD model of the dynamics of the irradiated upper atmosphere. 


\section{References}

Arras, P., \& Bildsten, L. 2006, ApJ, 650, 394

Arras, P., \& Socrates, A. 2009, arXiv:0901.0735

Ballester, G. E., Sing, D. K., \& Herbert, F. 2007, Nature, 445, 511

Batygin, K., \& Stevenson, D. J. 2010, ApJ, 714, L238

Ben-Jaffel, L. 2008, ApJ, 688, 1352

Blandford, R. D., \& Payne, D. G. 1982, MNRAS, 199, 883

Braginskii, S. I. 1965, Reviews of Plasma Physics , 1, 205

Charbonneau, D., Brown, T. M., Noyes, R. W., \& Gilliland, R. L. 2002, ApJ, 568, 377

Christensen, U. R. 2009, Space Science Reviews, 71

Christensen, U. R., Holzwarth, V., \& Reiners, A. 2009, Nature, 457, 167

Christie, D., Arras, P., \& Li, Z.-Y. 2013, ApJ, 772, 144

Cohen, O., Kashyap, V. L., Drake, J. J., et al. 2011, ApJ, 733, 67

Dalgarno, A., \& McCray, R. A. 1972, ARA\&A, 10, 375 
Dungey, J. W. 1961, Physical Review Letters, 6, 47

Ehrenreich, D., Lecavelier Des Etangs, A., Hébrard, G., Désert, J.-M., Vidal-Madjar, A., McConnell, J. C., Parkinson, C. D., Ballester, G. E., \& Ferlet, R. 2008, A\&A, 483, 933

Ehrenreich, D., \& Désert, J.-M. 2011, A\&A, 529, A136

Evans, C. R., \& Hawley, J. F. 1988, ApJ, 332, 659

Feldman, U., Behring, W. E., Curdt, W., Schuehle, U., Wilhelm, K., Lemaire, P., \& Moran, T. M. 1997, ApJS, 113, 195

Fortney, J. J., \& Nettelmann, N. 2009, Space Science Reviews, 115

France, K., Linsky, J. L., Yang, H., Stocke, J. T., \& Froning, C. S. 2011, Ap\&SS, 262

García Muñoz, A. 2007, Planet. Space Sci., 55, 1426

García-Segura, G., Langer, N., Różyczka, M., \& Franco, J. 1999, ApJ, 517, 767

Gold, T. 1959, J. Geophys. Res., 64, 1219

Hapke, B. 1993, Topics in Remote Sensing, Cambridge, UK: Cambridge University Press, - c1993,

Hawley, J. F., \& Stone, J. M. 1995, Computer Physics Communications, 89, 127

Hayes, J. C., et al. 2006, ApJS, 165, 188

Heinemann, M., \& Olbert, S. 1978, J. Geophys. Res., 83, 2457

Henry, G. W., Marcy, G. W., Butler, R. P., \& Vogt, S. S. 2000, ApJ, 529, L41 3

Heyvaerts, J., \& Norman, C. 1989, ApJ, 347, 1055 
Ip, W.-H., Kopp, A., \& Hu, J.-H. 2004, ApJ, 602, L53

Jensen, A. G., Redfield, S., Endl, M., et al. 2011, ApJ, 743, 203

Jensen, A. G., Redfield, S., Cochran, W. D., et al. 2011, Bulletin of the American Astronomical Society, \#218.06

Knutson, H. A., Charbonneau, D., Noyes, R. W., Brown, T. M., \& Gilliland, R. L. 2007, ApJ, 655, 564

Kopal, Z. 1978, Astrophysics and Space Science Library, 68,

Koskinen, T. T., Yelle, R. V., Lavvas, P., \& Lewis, N. K. 2010, ApJ, 723, 116

Krasnopolsky, R., Li, Z.-Y., \& Blandford, R. 1999, ApJ, 526, 631

Krasnopolsky, R., Li, Z.-Y., \& Blandford, R. D. 2003, Ap\&SS, 287, 75

Lamers, H. J. G. L. M., \& Cassinelli, J. P. 1999, Introduction to Stellar Winds, by Henny J. G. L. M. Lamers and Joseph P. Cassinelli, pp. 452. ISBN 0521593980. Cambridge, UK: Cambridge University Press, June 1999.,

Lecavelier Des Etangs, A. 2008, HST Proposal, 11673

Lecavelier Des Etangs, A., Pont, F., Vidal-Madjar, A., \& Sing, D. 2008, A\&A, 481, L83

Lecavelier Des Etangs, A., et al. 2010, A\&A, 514, A72

Lecavelier des Etangs, A., Bourrier, V., Wheatley, P. J., et al. 2012, A\&A, 543, L4

Lin, D. N. C., Bodenheimer, P., \& Richardson, D. C. 1996, Nature, 380, 606 
[17] Line, M. R., Vasisht, G., Chen, P., Angerhausen, D., \& Yung, Y. L. 2011, ApJ, 738,32

[18] Linsky, J. L., Yang, H., France, K., et al. 2010, ApJ, 717, 1291

Livio, M. 1997, IAU Colloq. 163: Accretion Phenomena and Related Outflows, 121, 845

Lovelace, R. V. E., Mehanian, C., Mobarry, C. M., \& Sulkanen, M. E. 1986, ApJS, 62,1

Lubow, S. H., \& Shu, F. H. 1975, ApJ, 198, 383

Mac Low, M.-M. 1999, ApJ, 524, 169

Martos, M., Yañez, M., Hernandez, X., Moreno, E., \& Pichardo, B. 2004, Journal of Korean Astronomical Society, 37, 199

Martos, M., Hernandez, X., Yáñez, M., Moreno, E., \& Pichardo, B. 2004, MNRAS, 350, L47

Mestel, L. 1968, MNRAS, 138, 359

Mestel, L., \& Spruit, H. C. 1987, MNRAS, 226, 57

Mouschovias, T. C., \& Paleologou, E. V. 1980, ApJ, 237, 877

Murray-Clay, R. A., Chiang, E. I., \& Murray, N. 2009, ApJ, 693, 23

Murray, C. D., \& Dermott, S. F. 2000, Solar System Dynamics, by C.D. Murray and S.F. Dermott, Cambridge, UK: Cambridge University Press, 2000.

Neubauer, F. M. 1998, J. Geophys. Res., 103, 19843 
Noyes, R. W., \& Kalkofen, W. 1970, Sol. Phys., 15, 120

Okamoto, I. 1974, MNRAS, 166, 683

Osterbrock, D. E., \& Ferland, G. J. 2006, Astrophysics of gaseous nebulae and active galactic nuclei, 2nd. ed. by D.E. Osterbrock and G.J. Ferland. Sausalito, CA: University Science Books, 2006

Perna, R., Menou, K., \& Rauscher, E. 2010, arXiv:1003.3838

Pneuman, G. W., \& Kopp, R. A. 1971, Sol. Phys., 18, 258

Preusse, S., et al. 2005, A\&A, 434, 1191

Preusse, S., Kopp, A., Büchner, J., \& Motschmann, U. 2007, Planet. Space Sci., 55, 589

Ray, T. P. 2007, IAU Symposium, 243, 183

Redfield, S., Endl, M., Cochran, W. D., \& Koesterke, L. 2008, ApJ, 673, L87

Reiners, A., \& Christensen, U. R. 2010, A\&A, 522, A13

Rybicki, G. B., \& Lightman, A. P. 1979, New York, Wiley-Interscience, 1979. 393 p.,

Saar, S. H., et al. 2008, IAU Symposium, 249, 79

Sánchez-Lavega, A. 2004, ApJ, 609, L87

Schunk, R. W., \& Nagy, A. F. 2004, Ionospheres

Shkolnik, E., Bohlender, D. A., Walker, G. A. H., \& Collier Cameron, A. 2008, ApJ, 676,628 
Sedov, L. I. 1959, Similarity and Dimensional Methods in Mechanics, New York: Academic Press, 1959,

Sing, D. K., Vidal-Madjar, A., Désert, J.-M., Lecavelier des Etangs, A., \& Ballester, G. $2008, \mathrm{ApJ}, 686,658$

Snellen, I. A. G., Albrecht, S., de Mooij, E. J. W., \& Le Poole, R. S. 2008, A\&A, 487, 357

Southworth, J. 2010, MNRAS, 408, 1689

Spruit, H. C. 1996, NATO ASIC Proc. 477: Evolutionary Processes in Binary Stars, 249

Spruit, H. C. 1996, arXiv:astro-ph/9602022

Stone, J. M., Mihalas, D., \& Norman, M. L. 1992, ApJS, 80, 819

Stone, J. M., \& Norman, M. L. 1992, ApJS, 80, 791

Stone, J. M., \& Norman, M. L. 1992, ApJS, 80, 753

Stone, J. M. 1999, Journal of Computational and Applied Mathematics, 109, 261

Stone, J. M., \& Proga, D. 2009, ApJ, 694, 205

Spruit, H. C. 1996, NATO ASIC Proc. 477: Evolutionary Processes in Binary Stars, 249

Tian, F., Toon, O. B., Pavlov, A. A., \& De Sterck, H. 2005, ApJ, 621, 1049

Torres, G., Winn, J. N., \& Holman, M. J. 2008, ApJ, 677, 1324

Trammell, G. B., Arras, P., \& Li, Z.-Y. 2011, ApJ, 728, 152 
Trammell, G. B., Arras, P., \& Li, Z. 2013, in prep.

Vidal-Madjar, A., et al. 2008, ApJ, 676, L57

Vidal-Madjar, A., et al. 2003, Nature, 422, 143

Vidal-Madjar, A., Désert, J.-M., Lecavelier des Etangs, A., Hébrard, G., Ballester, G. E., Ehrenreich, D., Ferlet, R., McConnell, J. C., Mayor, M., \& Parkinson, C. D. 2004, ApJ, 604, L69

Woitas, J., et al. 2005, A\&A, 432, 149

Wood, B. E., Redfield, S., Linsky, J. L., Müller, H.-R., \& Zank, G. P. 2005, ApJS, 159,118

Woods, T. N., Rottman, G. J., Bailey, S. M., Solomon, S. C., \& Worden, J. R. 1998, Sol. Phys., 177, 133

Wu, Y., \& Murray, N. 2003, ApJ, 589, 605

Yelle, R. V. 2004, Icarus, 170, 167 